\title{
Large Scale Oceanic Circulation and Fluxes of Freshwater, Heat, Nutrients and Oxygen
}

\author{
by \\ Alexandre Similien Ganachaud \\ Engineer, Ecole Supérieure d'Electronique de l'Ouest, Angers, France \\ Submitted in partial fulfillment of the requirements for the degree of \\ Doctor of Science \\ at the \\ MASSACHUSETTS INSTITUTE OF TECHNOLOGY \\ and the \\ WOODS HOLE OCEANOGRAPHIC INSTITUTION
}

December 1999

(C) Alexandre S. Ganachaud, 1999

The author hereby grants to MIT and WHOI permission to reproduce paper and electronic copies of this thesis in whole or in part and to distribute them publicly.

Signature of Author

Joint Program in Physical Oceanography Massachusetts Institute of Technology Woods Holg Ocęanogtaphäc Institution December 16, 1999

Certified by

Carl Wunsch

Cecil and Ida Green Professor of Physical Oceanography Massachusetts Institute of Technology Thesis Supervisor

Accepted by

W. Brechner Owens

Chairman, Joint Committee for Physical Oceanography Massachusetts Institute of Technology Woods Hole Oceanographic Institution 
Large Scale Oceanic Circulation and Fluxes of Freshwater, Heat, Nutrients and Oxygen

by

Alexandre Similien Ganachaud

Submitted to the MIT/WHOI Joint Program in Physical Oceanography on December 16, 1999, in partial fulfillment of the requirements for the degrees of

Doctor of Science 


\title{
Large Scale Oceanic Circulation and Fluxes of Freshwater, Heat, Nutrients and Oxygen
}

\author{
by \\ Alexandre Similien Ganachaud \\ Submitted in partial fulfillment of the \\ requirements for the degrees of \\ Doctor of Science
}

\begin{abstract}
A new, global inversion is used to estimate the large scale oceanic circulation based on the World Ocean Circulation Experiment and Java Australia Dynamic Experiment hydrographic data. A linear inverse "box" model is used to combine consistently the transoceanic sections. The circulation is geostrophic with an Ekman layer at the surface and oceanic layers defined by neutral surfaces. Near-conservation of mass, salt and top-to-bottom silica is required and, in addition, heat and the phosphate-oxygen combination $\left(170\left[\mathrm{PO}_{4}\right]+\left[\mathrm{O}_{2}\right]\right)$ are conserved in layers that are not in contact with the surface. A globally-consistent solution is obtained for a depth-independent adjustment to the thermal wind field, freshwater flux divergences, the Ekman transport, and the advective and diffusive dianeutral fluxes between layers. A detailed error budget permits calculation of statistical uncertainties, taking into account both the non-resolved part of the solution and the systematic errors due to the temporal oceanic variability. The estimated water mass transports during the WOCE period (1985-1996) are generally similar to previous published estimates. However, important differences are found. In particular, the inflow of bottom waters into the Pacific Ocean is smaller than in most previous estimates. Utilization of property anomaly conservation constraints allows the estimation of significant dianeutral diffusivities in deep layers, with a global average of $3 \pm 1 \mathrm{~cm}^{2} \mathrm{~s}^{-1}$ north of $30^{\circ} \mathrm{S}$. Dianeutral transfers indicate that about $20 \mathrm{~Sv}$ of bottom water is formed in the Southern Ocean. Significant oceanatmosphere heat fluxes are found, with a global heating of $2.3 \pm 0.4 \mathrm{PW}$ in the tropical band and a corresponding cooling at high latitudes. The signature of a large-scale average export production is found for nutrients in several temperate regions. Despite the large uncertainties, the production magnitudes are consistent with independent measurements from sediment traps and isotopic data. Net nutrient sources or sinks are found in several regions, suggesting either transport of dissolved organic matter or a seasonal alias. Oxygen indicates large exchanges with the atmosphere, with intake at high latitudes and outgassing/remineralization at low latitudes.
\end{abstract}

Thesis Supervisor: Carl Wunsch

Title: Cecil and Ida Green Professor of Physical Oceanography Massachusetts Institute of Technology 


\section{Contents}

Abstract $\quad 3$

$\begin{array}{lr}\text { Acknowledgments } & 8\end{array}$

$\begin{array}{ll}\text { Overview } & 10\end{array}$

1 Data and Model 14

1.1 The data $\ldots \ldots \ldots \ldots \ldots \ldots \ldots \ldots$

1.1.1 Hydrographic sections . . . . . . . . . . . . . . . . 14

1.2 Model formulation . . . . . . . . . . . . . . . . . . 16

1.2.1 Flux through a single hydrographic section . . . . . . . . . 18

1.2 .2 Conservation equations $\ldots \ldots \ldots \ldots \ldots \ldots$

1.2 .3 Ekman transport . . . . . . . . . . . . . . . . . . 21

1.2.4 Anomaly Equations . . . . . . . . . . . . . . . 22

1.3 The Gauss-Markov estimator . . . . . . . . . . . . . . . 25

2 The Error Budget of Inverse Box Models $\quad 27$

2.1 Introduction . . . . . . . . . . . . . . . . . 27

2.2 Model Error . . . . . . . . . . . . . . . . . . . 30

2.2 .1 The general circulation model $(\mathrm{GCM}) \ldots \ldots \ldots$

2.2 .2 Oceanic response to Ekman fluctuations . . . . . . . . . . . . 31

2.2 .3 Baroclinic variability . . . . . . . . . . . . . . 33

2.2 .4 Silica Variability . . . . . . . . . . . . . . . . . . . . 41

2.3 Measurement noise . . . . . . . . . . . . . . . . 46 
2.3 .1 Internal waves $\ldots \ldots \ldots \ldots \ldots \ldots$

2.3 .2 Geostrophy . . . . . . . . . . . . . . . . 49

2.3 .3 Bottom triangle. . . . . . . . . . . . . . 50

2.3.4 Mean Ekman transport uncertainty . . . . . . . . . . . 56

2.4 Reference level velocity variability $\ldots \ldots \ldots \ldots$

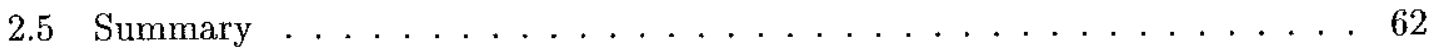

2.6 Application to the global inversion ................ 66

3 Physical circulation $\quad 69$

3.1 The Indian Ocean $\ldots \ldots \ldots \ldots \ldots \ldots$

3.1 .1 Introduction . . . . . . . . . . . . . . 71

3.1 .2 Indian Ocean Problems . . . . . . . . . . . . 72

3.1.3 Model constraints . . . . . . . . . . . . . 75

3.1.4 Indian Ocean Circulation: standard solution . . . . . . . . . 80

3.1 .5 The Mozambique channel . . . . . . . . . . . . . . 87

3.1.6 The Pacific Indian Throughflow (PIT) . . . . . . . . . . . 88

3.1.7 Full Property Equations and PO constraints . . . . . . . . . . . 89

3.1 .8 Indian Ocean summary . . . . . . . . . . . . . . 90

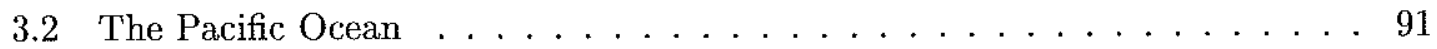

3.2 .1 Introduction . . . . . . . . . . . . . 91

3.2 .2 Model constraints . . . . . . . . . . . . . . 92

3.2 .3 Pacific Ocean circulation . . . . . . . . . . . . . 97

3.2 .4 Pacific circulation summary . . . . . . . . . . . . . . 104

3.3 The Atlantic Ocean . . . . . . . . . . . . . . . . . 106

3.3 .1 Introduction . . . . . . . . . . . 106

3.3 .2 Model constraints . . . . . . . . . . . . . . 107

3.3 .3 Atlantic Ocean Circulation . . . . . . . . . . . . 113

3.3 .4 Atlantic circulation summary . . . . . . . . . . . . . . . 124

3.4 The Southern Ocean . . . . . . . . . . . . . . . 126

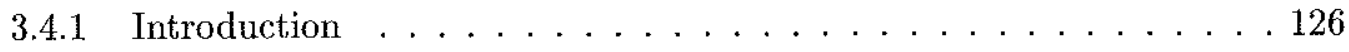


3.4 .2 Model constraints . . . . . . . . . . . . . . . 127

3.4.3 Southern Ocean Circulation . . . . . . . . . . . . 130

3.4.4 Southern Ocean circulation summary . . . . . . . . . . 136

3.5 Global circulation summary . . . . . . . . . . . . . 137

4 Global Property Fluxes and Divergences 139

4.1 Freshwater flux divergences $\ldots \ldots \ldots \ldots$. . . . . . . . . . . . . . . . . . . .

4.2 Heat transports . . . . . . . . . . . . . . . . . . . 144

4.2.1 Global energy transports and heat transfers . . . . . . . . . . 145

4.3 Export production and nutrient cycle . . . . . . . . . . . 156

4.3 .1 Silica . . . . . . . . . . . . . . . . . . 157

4.3.2 Nitrate and Phosphate . . . . . . . . . . . . . 163

4.3.3 Discussion of nutrient fluxes and divergences $\ldots \ldots . . . . . .167$

4.4 Oxygen fluxes and divergences $\ldots \ldots \ldots \ldots$

4.4 .1 The oxygen cycle . . . . . . . . . . . . . . . . 169

4.4 .2 Oxygen fluxes and divergences $\ldots \ldots \ldots$. . . . . . . . . . . . . .

5 Conclusions $\quad 176$

5.1 Global summary . . . . . . . . . . . . . . 176

5.2 Discussion and perspectives . . . . . . . . . . . . . . 178

5.2 .1 New data . . . . . . . . . . . . . . . . . 179

5.2 .2 Improvements to the present model . . . . . . . . . . . 180

5.2 .3 Improving the dynamics . . . . . . . . . . . . . . . 181

$\begin{array}{lr}\text { Appendices } & 182\end{array}$

A Ageostrophy in the General Circulation Model $\quad 182$

$\begin{array}{lll}\text { B } & \text { Model Error in a Two-dimensional Ocean } & 188\end{array}$

$\begin{array}{ll}\text { C A priori mass uncertainties } & 192\end{array}$

D Standard solution absolute velocities and cumulative transports $\quad 195$ 
E Standard solution property fluxes

References

250 


\section{Acknowledgments}

I feel privileged to have had an advisor who was permanently available, encouraging and trustful. I truly enjoyed working with Carl. His enthusiasm and physical intuition were stimulating, and this thesis was greatly enriched by his suggestions and constructive comments. Carl is generous with his ideas, but he would always encourage me to pursue my own. He reviewed and commented on many drafts of this thesis, always in record time $\cdots$ which is so appreciable for a graduate student!

I was impressed by the warm welcome and interest of scientists at both WHOI and MIT. Their doors were always open for a discussion. In particular, thanks to my committee members John Toole, Bruce Warren, Jochem Marotzke, Dave Glover, Nelson Hogg for their careful reading of the thesis and numerous suggestions. John Toole suggested the use of anomaly equations, which had an important impact on the thesis results. Bill Jenkins provided helpful advice at the outset of this work. The part on the Indian Ocean benefited from insightful comments by Paul Robbins, Gregory Johnson, Harry Bryden, Michèle Fieux and Trevor McDougall.

Alison Macdonald's help was essential in my beginnings with inverse modelling. I also benefited from numerous conversations with Galen McKinley and Mick Follows. Steve Jayne and Robin Tokmakian provided the GCM output fields for the simulation of Chapter 2. Diana Spiegel did a large part of the data treatment and produced Figs.1-1, 3-1, 3-5, 3-9, and 3-14. Bud Brown produced Fig. 5-1. Chris Holloway calculated the neutral surface areas and help with the data treatment. Thanks also to Linda Meinke and Charmaine King for their help with computers.

Many people have made my life at MIT and WHOI extremely enjoyable. Thanks in particular to Christophe, Juan, Galen, Elodie, Valerie, Kees, Vikas, Richard, Xiaoyun, Misha, Markus, François, Albert, Steve, Brian, Lous, Stephanie, Allistair, Chris, Hua, Helen and Lisa for creating a warm and stimulating atmosphere.

This work was supported in part by the Jet Propulsion Laboratory/CALTECH (contract \# 958125), and by gifts from Ford, General Motors, and Daimler-Chrysler to MIT's Climate Modelling Initiative. I used extensively the SEAWATER routines of P. Morgan. 
The WHPO data center was very helpful to provide most of the data. I am grateful to the Principal Investigators who let me use their data, either directly or through the WHPO Center when public. Thanks in particular to B. Warren, G. Johnson, W. Nowlin, J. Toole, N. Bray, J.Sprintall (Indian Ocean); L. Talley, D. Roemmich, J. Swift, M. McCartney, H. Bryden, Brady, J. Toole (Pacific Ocean); P. Koltermann, V. P. Tereschenkov, G. Parilla, R. Millard, M. Arhan, A. Morlière, C. Oudot, C. Colin, Y. Gouriou, G. Siedler, T. Mueller, R. Onken, P. Saunders (Atlantic Ocean); W. Roether, P.Lemke, Y.-H. Park, N. Metzl and C. Jeandel, M. McCartney, A. Gordon, S.Rintoul, J.Bullister, and G. Johnson (Southern Ocean). The data from the Franco-Indonesian JADE cooperation were provided by M. Fieux and A. G. Ilahude (PIs); B. Coste and C. Coatanoan (nutrients). I am grateful as well to the technicians and crew who produced this high quality data set.

I thank my parents for taking my sister, my brother and me on a sailboat for five years when I was 9-that was the discovery of the Ocean. Lastly and most importantly, thanks to my wife Doiniţa, whose love, support and confidence guided me throughout the turbulent meanders of my graduate research. 


\section{Overview}

By its ability to transport important quantities of energy and dissolved carbon dioxide, the ocean plays a major role in climate regulation. One of the main objectives of the World Ocean Circulation Experiment (WOCE) was the determination of such transports.

Oceanic surface waters are heated in low latitudes and transported by ocean currents towards higher latitudes. As a result, the ocean carries about the same amount of thermodynamic energy ${ }^{1}$ as does the atmosphere from the equator to the poles. Estimates of oceanic energy transports can be obtained by integration of the residuals from atmospheric models, but the uncertainties associated with this method are relatively large (e.g., Keith, 1995). Alternatively, the energy transport can be derived from oceanic currents across transoceanic hydrographic sections. This later method is believed to be more accurate, and allows uncertainty calculations (e.g., Wijffels et al., 1999; Robbins and Toole, 1997; Macdonald and Wunsch, 1996; Bryden et al., 1991; Rintoul and Wunsch, 1991; Hall and Bryden, 1982, and many others).

Similarly, carbon dioxide $\left(\mathrm{CO}_{2}\right)$ and oxygen $\left(\mathrm{O}_{2}\right)$ are absorbed and released at the ocean surface. Atmospheric $\mathrm{CO}_{2}$ concentration is one of the key variables that affect the Earth's temperature and climate. The ocean is believed to be an important sink of anthropogenic $\mathrm{CO}_{2}$, in particular, the photosynthetic activity in the surface layer that consumes dissolved $\mathrm{CO}_{2}$ fixes carbon and exports it towards the deep layers, through this "biological pump" effect (Sarmiento et al., 1990). The resulting depletion of $\mathrm{CO}_{2}$ in the surface layer increases the amount of atmospheric $\mathrm{CO}_{2}$ transferred to the ocean relative to a hypothetical ocean with no biological activity. Simulations suggest that this process may be lowering the atmospheric $\mathrm{CO}_{2}$ concentration by a factor of 2 (e.g., Sarmiento and Toggweiler, 1984).

The $\mathrm{CO}_{2}$ export is thus intimately related to nutrient transport in the ocean, which can be calculated from hydrographic data. Nutrients are consumed by planktonic organisms in the surface layers of the ocean. They in turn are converted to particulate and organic matter, go through the food chain and end up in sinking fecal pellets and skeletons.

\footnotetext{
${ }^{1}$ Often mislabeled "heat".
} 
Some of the particles are recycled in the euphotic zone (regenerated production) while the remainder sink towards the deep oceanic layers (export production) where most are remineralized. These dissolved nutrients then flow slowly with the currents until they reach the surface to again support production.

Existing estimates of new and export production are very uncertain. Because of large spatial and temporal variability in biological activity, direct measurements of export production and remineralization are difficult to interpret. Most estimates are local (e.g., Sarmiento et al., 1990; Spitzer and Jenkins, 1989) and rely upon uncertain assumptions, such as a one-dimensional balance over the water column. On the other hand, it is possible, in principle, to estimate new and export production from an accurate dissolved nutrient budget in the ocean. An estimate of oceanic nutrient transports should reveal, in the presence of export production, a dissolved nutrient depletion in the euphotic zone and a dissolved nutrient supply at deeper levels.

In this thesis, I seek a global circulation scheme that is consistent with oceanic dynamics and simple biology and geochemistry. The benefits are two-fold: on the one hand, the dynamics provide information about the nutrient fluxes; on the other hand, the nutrient fluxes are able to provide constraints on the circulation. This is a first attempt to use the WOCE hydrographic data in such a global and consistent framework.

Inverse methods provide convenient tools to quantify an ocean circulation that obeys simple conservation statements. Since the pioneering works of Riley (1951) and later Wunsch (1978), steady geostrophic box inversions have been extensively used in most oceanic regions (see Wunsch, 1996 for a review). The method used here follows the earlier global calculations of Macdonald and Wunsch (1996) and Macdonald (1998) with a number of significant improvements, including the following:

1. The present data consist almost entirely of recent, high-quality hydrographic sections from the WOCE survey.

2. A more quantitative estimate of the uncertainties in property transports is obtained through an analysis of $1 / 4^{\circ}$ nominal lateral resolution General Circulation Model run at the US Naval Post Graduate School (see Semtner and Chervin, 1992; Stammer et 
al., 1996). Temporal variability dominates the errors of inversions done using synoptic section data. An important example is the large estimated potential deviation of the silica flux from its long-term average value when computed from any particular section.

3. The use of conservation equations in terms of property anomalies (e.g., McDougall, 1991a; Wijffels, 1993) rather than full property values themselves-but based upon making explicit the necessary assumptions concerning the error covariance between mass constraints and property constraints (Wunsch, 1996, p.273).

4. Use of neutral surfaces (McDougall, 1987; Jackett and McDougall, 1997) to define layer boundaries with calculation, as part of the inversion, of the dianeutral transfers, dianeutral diffusion of tracers, freshwater fluxes along with the reference velocities and ageostrophic transport adjustment in the upper-most layer (that I ascribe to the Ekman transport uncertainty).

5. The Ekman transport in the surface layers is derived from the mean wind fields from the NCEP reanalysis (Kalnay et al. 1996).

6. The nutrient and oxygen fluxes and divergences are analyzed in relation to biogeochemical cycles.

The major part of the thesis concerns the estimation of a globally consistent large-scale oceanic circulation. In the Atlantic Ocean, my estimated water mass transports during the WOCE period (1985-1996 globally, although Atlantic sections span 1991-1993) are similar to previously published estimates. However, differences are found, noticeably in the Pacific Ocean, where the inflow of bottom waters is smaller than most previous estimates.

By imposing stringent heat and salt conservation constraints, property anomalies allow the estimation of consistent and significant dianeutral transfers and diffusivities, in contrast with many previous box inversions. Deep dianeutral advection and diffusivities that are required by the tracer fluxes (mainly heat and salt) average respectively $0.1 \pm 0.04 \times 10^{-4} \mathrm{~cm} \mathrm{~s}^{-1}$ and $3 \pm 1 \mathrm{~cm}^{2} \mathrm{~s}^{-1}$. Larger diffusivities of about $20 \mathrm{~cm}^{2} \mathrm{~s}^{-1}$ are found in bottom layers. 
Energy transports evidence significant ocean-atmosphere heat fluxes, with a global heating of $2.3 \pm 0.4 \mathrm{PW}$ in the tropical band. Most of the cooling occurs in the northern hemisphere $(1.7 \pm 0.2 \mathrm{PW})$ while in the southern hemisphere only $0.7 \pm 0.3 \mathrm{PW}$ is restored to the atmosphere. Nutrient residuals are in general uncertain. There is an interesting, but weak indication in several regions of nutrient consumption in the surface layers and deeper remineralization. The large uncertainties are due to time variability in the measured temperature and salinities and to the limited vertical resolution of the model. Nevertheless, the results provide an upper bound on the average remineralization rate in several depth ranges. Oxygen residuals indicate large and significant exchange with the atmosphere, with oxygen intake at high latitudes and outgassing at low latitudes. Significant oxygen utilization rate estimates indicate remineralization in subsurface layers in several regions.

The remaining uncertainty in estimating the global oceanic circulation and fluxes is dominated by oceanic variability in the density and other properties. In particular, the large variability of the Pacific-Indonesian Throughflow degrades the property budgets in both the Indian and Pacific Oceans. Similarly, the variable North Brazil Current impacts the Atlantic's budget.

The thesis is organized as follows. Firstly, the method used to estimate the circulation and transports is described (Chapter 1). A detailed analysis of the uncertainties due to oceanic variability and measurement noise follows in Chapter 2. My estimate of the physical oceanic circulation for each oceanic basin is described in Chapter 3 . The data treatment and model constraints that are specific to each ocean are presented there. In Chapter 4, I analyze the large scale fluxes of freshwater, heat, nutrients and oxygen in a global context. Chapter 5 gives a general summary of the thesis and a discussion with conclusive remarks and perspectives for the future. 


\section{Chapter 1}

\section{Data and Model}

\subsection{The data}

\subsubsection{Hydrographic sections}

The circulation estimate is mostly based upon World Ocean Circulation Experiment (WOCE) hydrographic sections with high-quality water sample data. The sections that define the model are displayed on Figure 1-1 and listed on Table 1.1. In the Atlantic at $36^{\circ} \mathrm{N}$, we attempted to use the older AT109 data (Roemmich and Wunsch, 1985) from 1981 because the 1993 data made available to us (Sokov et al., 1998) showed a large bias in the mutrients and oxygen. However, the 1981 data turned out to be incompatible with the WOCE lines A5 and A2, producing large and significant residuals in the upper layers, and it was decided to use neither section at this latitude. The Pacific section $\mathrm{P} 4\left(10^{\circ} \mathrm{N}\right)$ was also removed from the model because it caused large mass residuals and downwelling at intermediate levels, probably because of baroclinic variability in the Mindanao Current that was aliased in this section (S. Wijffels, personal communication, 1999).

Given the data, the model solution, at best, represents an average of the circulation for the 1989-1996 period in most oceans, except in the North Pacific where the sections were taken earlier, in 1985. Many sections are not public or were not public when I started this work. They were used with the consent of the principal investigators and I thank them for letting me use their data, either by making them publicly available or by providing them 


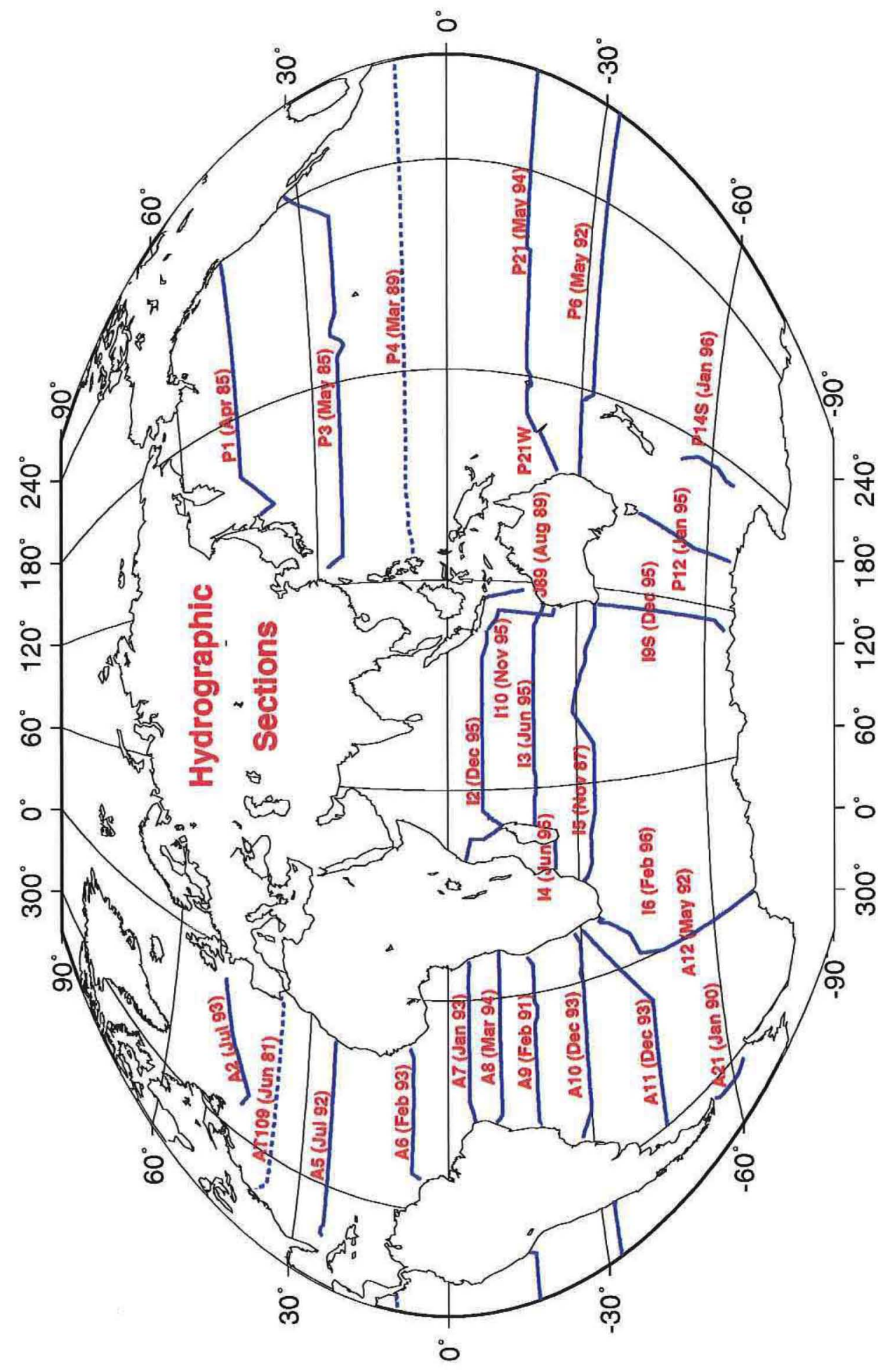

Figure 1-1: Hydrographic sections. The sections are WOCE, pre-WOCE and JADE and cover the period 1985-1996. 


\begin{tabular}{|c|c|c|}
\hline Section & Date & Reference \\
\hline A2 Gauss 226 & Jul. 93 & Koltermann et al. (1999) \\
\hline AT109-36 & Jun. 81 & Roemmich and Wunsch (1985) \\
\hline A5 & Jul. 92 & Parilla et al. (1994) \\
\hline A6 & Feb. 93 & Arhan et al. (1998) \\
\hline $\mathrm{A} 7$ & Jan. 93 & Oudot et al. (1998) \\
\hline A8 & Mar. 94 & \multirow{3}{*}{ Siedler et al. (1996) } \\
\hline A9 & Feb. 91 & \\
\hline $\mathrm{A} 10$ & Dec. 93 & \\
\hline A11 & Dec. 93 & Saunders and King (1995) \\
\hline A21 & Jan. 90 & Roether et al. (1993) \\
\hline A12 & May 92 & Lemke (1994) \\
\hline I6 & Feb. 96 & Park et al. (1999) \\
\hline I5 & Nov. 87 & Toole and Warren (1993) \\
\hline I4 & Jun. 95 & \\
\hline $\mathrm{I} 3$ & Jun. 95 & \\
\hline $\mathrm{I} 2$ & Dec. 95 & Johnson et al. (1998) \\
\hline $\mathrm{I} 10$ & Nov. 95 & Bray et al. (1997) \\
\hline JADE 1989 & Aug. 89 & Fieux et al., 1994, 1996 \\
\hline I9S & Dec. 95 & Hufford et al. (1997) \\
\hline $\mathrm{P} 12$ & Jan. 95 & Rosenberg et al. (1997) \\
\hline P14S & Jan. 96 & McTaggart and G. Johnson (1997) \\
\hline P6 & May 92 & Tsimplis et al. (1998); Wijffels et al. (1999) \\
\hline P21 & May 94 & Banks et al. (1995) \\
\hline $\mathrm{P} 4$ & Mar. 89 & Wijffels et al. (1996) \\
\hline P3 & May 85 & Roemmich et al. (1991) \\
\hline P1 & Apr. 85 & Talley et al. (1991) \\
\hline
\end{tabular}

Table 1.1: Hydrographic sections

directly to me. Their respective names are listed in the physical description of individual oceans in Chapter 3 and in the acknowledgments. More details on the data are provided in Chapter 3.

\subsection{Model formulation}

Inverse methods applied to the determination of oceanic circulation are now widely documented in the literature. I used here the so called "inverse box model" described by Wunsch (1978) and subsequently used in various studies (e.g., Wunsch, 1982; Rintoul and Wunsch, 1991; Wijffels, 1993; Macdonald and Wunsch, 1996; Tsimplis et al., 1998, 


\begin{tabular}{|l|c|c|}
\hline & Top-to-Bottom & within individual layers \\
\hline Mass & yes & yes \\
\hline Thermodynamic energy (heat) & no & below surface \\
\hline Salinity & yes & yes \\
\hline Silica & yes & no \\
\hline "NO" $\left(9.1 \mathrm{NO}_{3}+\mathrm{O}_{2}\right)$ & no & no \\
\hline "PO" $\left(170 \mathrm{PO}_{4}+\mathrm{O}_{2}\right)$ & no & below surface \\
\hline
\end{tabular}

Table 1.2: Conservation constraints for the standard solution. Heat, salinity and "PO" use the anomaly formulation.

etc.). Wunsch (1996) gives a complete description of the various methods along with their statistical background.

The geostrophic circulation with a surface Ekman layer is required to conserve mass and other tracers within individual layers bounded by neutral surfaces (McDougall, 1987). Table 1.2 gives a summary of the different constraints and how they are applied. Conservation constraints take into account advective and diffusive exchanges between layers, hereafter referred to as dianeutral fluxes.

The thermodynamic energy (usually mislabeled "heat", e.g., Warren (1999)) and the phosphate-oxygen "PO" (170 $\mathrm{PO}_{4}+\mathrm{O}_{2}$, Anderson and Sarmiento, 1994) combination are approximately conserved in layers that are not in contact with the atmosphere. (The surface layer is non-conservative because of heat and oxygen exchanges with the atmosphere.) The value 170 for the PO Redfield ratio is controversial (e.g., Minster and Boulahdid, 1987 ; Anderson and Sarmiento, 1994). However, an experiment with a lower ratio of 140 showed that the circulation was not changed significantly. Conservation equations for the tracer "NO" $\left(9.1 \times \mathrm{NO}_{3}+\mathrm{O}_{2}\right.$; Broecker (1974)) are, in general, indistinguishable from the "PO" equations and I chose to conserve "PO" alone because the nitrogen cycle is the more complex one. The total (top-to-bottom) silica is approximately conserved, but silica residuals are allowed in individual layers to accommodate consumption and dissolution through the biological cycle (Section 4.3.1).

Bulk advective $\left(w^{*}\right)$ and diffusive $\left(\kappa^{*}\right)$ dianeutral transfers are allowed between the layers for each property and (Section 1.2.2). The same $w^{*}$ and $\kappa^{*}$ are used for all properties. By definition there is no diffusion in the mass equation (e.g., Pedlosky, 1987, page 10). (It 
is also possible to derive a density equation from the energy conservation equation, but this equation is redundant with heat and salt conservation). The bulk average property and property gradients on a neutral surface are calculated from the bounding sections. Freshwater flux divergences and adjustments to the Ekman transports in the surface layers are solved for as well (Section 1.2.3).

To better condition the system, property anomaly equations are used for salt, heat and "PO" (Section 1.2.4) but not for silica, i.e. Section 1.2.4. Solutions are obtained using the Gauss-Markov estimator so that the uncertainties include the null-space (the part of the solution that is not resolved by the constraints), in contrast with standard least-squares and singular value decomposition methods (Section 1.3). All conservation equations were weighted according to their a priori uncertainty that will be determined in the next chapter (Chapter 2).

\subsubsection{Flux through a single hydrographic section}

Consider a hydrographic section running in the zonal $(x)$ direction, composed of a set of stations, where temperature $(T)$, salinity $(S)$, nutrients and oxygen were measured over the water column. As the circulation is in geostrophic balance to a good approximation, the velocity field is obtained by integrating the thermal wind equation,

$$
v_{a b s}(x, z)=v_{\text {rel }}(x, z)+b(x)=-\frac{g}{\rho_{0} f} \int_{z_{0}(x)}^{z} \frac{\partial \rho}{\partial x} d z+b(x),
$$

where $z$ is the vertical coordinate; $x$ is the horizontal coordinate along the section; $z_{0}(x)$ is an arbitrary reference depth at each station pair; $v_{a b s}(x, z)$ is the absolute velocity, the sum of the velocity relative to the reference depth, $v_{r e l}(x, z)$ computed from the data, plus the reference velocity, $b(x)$, which is unknown ${ }^{1} . g$ is the local gravitational acceleration and $f$ is the Coriolis parameter. The surface formed by $z_{0}(x)$ is called the initial zero-velocity surface, or reference surface. Depending upon the section, this surface may be a straight line (reference level), a neutral surface, or may be specified for each pair according to the surrounding water properties. Both $b$ and $v$ are normal to the section and defined as the horizontal average between two consecutive stations (a station pair). Classically, the

\footnotetext{
${ }^{1}$ I have attempted to keep my notations consistent with Wunsch (1996).
} 
circulation was inferred by elaborate guesses of the reference surface, $z_{0}(x)$, at which $b(x)$ would vanish. The inverse box model is cast as providing an estimate of the adjustment, $\{b\}=\tilde{\mathbf{b}}$, to this classical a priori solution, along with an error covariance $\mathbf{P}$, which satisfies a number of requirements on the circulation and the a priori variance of $b$. These requirements may include, for instance, mass, salt and silica conservation.

The net transport of a tracer across the section is, by definition:

$$
\begin{aligned}
T & =\int_{\text {West }}^{\text {East }} d x \int_{B o t(x)}^{T o p(x)} d z\left[b(x)+v_{r e l}(x, z)\right] \rho(x, z) C(x, z) \\
& =T_{b}+\Gamma
\end{aligned}
$$

where $C$ is the tracer concentration per unit mass $\left(C=1\right.$ for mass), $T_{b}$ is the contribution from the reference velocities and $\Gamma$ is the contribution from the relative velocities. In the

discrete form, $T_{b}$ is a linear combination of the $b^{\prime} s, T_{b}=\mathbf{a}^{T} \mathbf{b}$, where the elements of $\mathbf{a}$ contain the integrals of $\rho C$ over the water column at each station pair and $\mathbf{b}$ is the column vector containing $b(x)$. $T$ may be the transport through an individual layer, $\operatorname{Top}(x)$ and $B o t(x)$ then defining the upper and lower boundaries of the layer.

\subsubsection{Conservation equations}

In steady-state, mass conservation (continuity) may be written as

$$
\nabla \cdot(\rho \mathbf{v})=0
$$

while a tracer conservation equation may be written as

$$
\nabla \cdot(\rho C \mathbf{v})=\nabla \cdot \kappa \nabla(\rho C)
$$

where $C$ is the tracer concentration; $v$ is the three-dimensional velocity vector, and $\kappa$ is the eddy diffusion tensor. In the present model, it is assumed that the spatial sampling along a section is fine enough to resolve most of the horizontal tracer transport due to eddies at a given time (the ergodicity assumption is discussed in the next chapter). The smallerscale lateral processes (including horizontal diffusion) are contained in the noise. One can integrate (1.4) over a layer bounded horizontally by two zonal hydrographic sections and 
land, and vertically between selected neutral surfaces and rearrange the terms to obtain

$$
\left[\int_{\text {West }}^{\text {East }} d x \int_{B o t(x)}^{\text {Top }(x)} d z \rho C v\right]_{\text {South }}^{\text {North }}=\left[\iint d x d y\left(\kappa \frac{\partial(\rho C)}{\partial z}-w \rho C\right)\right]_{B o t}^{T o p}
$$

where $[X]_{\text {South }}^{\text {North }} \doteq X^{\text {North }}-X^{\text {South }}, w$ is the velocity perpendicular to the top or bottom interface and $\kappa$ the dianeutral diffusivity. The left-hand-side can be calculated from the velocities across the sections, but the right-hand-side is not directly computable. The right-hand-side is decomposed as follows, assuming that the second order Reynolds terms are negligible:

$$
\iint d x d y\left(\kappa \frac{\partial(\rho C)}{\partial z}-w \rho C\right) \simeq A \times\left(\kappa \kappa^{*} \frac{\overline{\partial(\rho C)}}{\partial z}-w^{*} \overline{\rho C}\right),
$$

where the horizontal overbar denotes a horizontal average; $A$ is the horizontal area of a given neutral surface bounded by the sections, and $w^{*}, \kappa^{*}$ represent the equivalent average dianeutral velocity and diffusivity across the interface. Such a formalism assumes for $\kappa^{*}>0$ down-gradient turbulent fluxes in the dianeutral direction. Both $\overline{\rho C}$ and $\frac{\overline{\partial(\rho C)}}{\partial z}$ are calculated from the section data, that is, at the boundaries of each ocean sector. The horizontal area between sections, $A$, was calculated according to the neutral surface positions as derived from the Levitus (1994) climatology.

Using (1.3) and (1.6) and accounting for possible sources and sinks of each tracer, Eq. (1.5) may be written as

$$
\left[T_{b}\right]_{\text {South }}^{\text {North }}+A \times\left[w^{*} \overline{\rho C}-\kappa^{*} \frac{\overline{\partial(\rho C)}}{\partial z}\right]_{\text {Bot }}^{\text {Top }}=[-\Gamma]_{\text {South }}^{\text {North }}+Q
$$

where $Q$ is a possible source or sink of tracer $C$. ( $Q$ includes the freshwater flux in the mass budget and the Ekman flux divergence for all tracers including mass.) Eq. (1.7) is written in the discrete form as

$$
\begin{aligned}
\mathbf{a}^{T} \mathbf{b}+n=y & \\
\text { with } \mathbf{b} & =\left[\begin{array}{l}
\left\{b_{i}\right\}_{i=1, N \text { pair }} \\
\Delta T_{E k} \\
\left\{w_{k}^{*}\right\}_{k=1, N l a y-2} \\
\left\{\kappa_{k}^{*}\right\}_{k=1, N l a y-2} \\
\Delta F W
\end{array}\right] \text {. }
\end{aligned}
$$


$\mathbf{b}$ is the column vector containing the unknowns of the system, respectively, the reference velocities at each station pair $\left\{b_{i}\right\}$; the adjustment to the Ekman transport (see below); the dianeutral fluxes across each interface $\left\{w_{k}^{*}\right\}$ and $\left\{\kappa_{k}^{*}\right\}$; and the freshwater flux in the mass equation, $\Delta F W$. The term $y$ contains the relative transport divergence $(\Gamma)$ and the source term $Q$. A noise $n$ has been introduced in Eq.1.8 as the equation cannot be satisfied exactly with real data. A quantitative discussion of the noise follows in Chapter 2 . A similar equation is added for each layer, for the total flow, and for several tracers including mass $(C=1)$. All the equations of the form (1.8) may be grouped to form a single matrix equation

$$
\mathbf{A b}+\mathbf{n}=\mathbf{y}
$$

where $\mathbf{A}$ is now a matrix of size $M \times N$, where $M$ is the number of equations and $N$ is the number of unknowns. ( $\mathbf{n}$ and $\mathbf{y}$ are $M \times 1$.) Constraints on the net flux through a particular set of station pairs and layers (e.g., Florida Strait flux, topographic constraints) are added by choosing the appropriate elements of $\mathbf{a}$ and $\mathbf{y}$.

\subsubsection{Ekman transport}

The Ekman transport is included in (1.9). Annual variations in the Ekman transport can be large (e.g., Levitus, 1988). However, the relevant quantity to our time-average circulation model is the time-average Ekman transport. The Ekman transport was obtained from an average wind stress of the NCEP/NCAR reanalysis (Kalnay et al., 1996) over the period 1992 to $1997^{2}$. In the model formulation, the Ekman transport is decomposed into two contributions:

$$
T_{E k}^{\text {true }}=T_{E k}^{\text {init }}+\Delta T_{E k}
$$

where $T_{E k}^{i n i t}$ is the initial Ekman transport, calculated from the climatology, and $\Delta T_{E k}$ is the correction required by the conservation equations. $\Delta T_{E k}$ is included in $\mathbf{b}$ and solved for in the inversion. The a priori uncertainty on the Ekman transport, $\Delta T_{E k}$, is $50 \%$ of the

\footnotetext{
${ }^{2}$ This period does not exactly overlap with the hydrographic data. The model-derived correction to the Ekman transport is assumed to accommodate the temporal differences.
} 
initial value, with a lower bound of $1 \mathrm{~Sv}$ (see the discussion in Chapter 2, Section 2.3.4). The value for initial Ekman transports $T_{E k}^{i n i t}$ and for the correction after inversion $\Delta T_{E k}$ are given for each ocean in Chapter 3 (Tables 3.2, page 78; 3.6, page 95); 3.11, and page 112 .

The Ekman transport of tracers is estimated with the product $\left(T_{E k}+\Delta T_{E k}\right) \times \bar{C}$ where $\bar{C}$ is the average property concentration in the upper layer(s) on each full section. In the places where a layer outcrops along a section, the Ekman transport was calculated over the interval of outcrop. But $\bar{C}$ was taken as the average over the whole layer. This simple calculation excludes the effects of correlations between horizontal property variations and Ekman transport within the surface layer. It is however a good approximation for the net temperature transport which is mainly controlled by the vertical temperature difference between the surface and deep ocean, horizontal gradients playing a minor role (S. Jayne and J.Marotzke, personal communication, 1998). Equivalently, the nutrient fluxes are not much affected by the approximation because nutrients are generally depleted in the upper layers at low latitudes, where the Ekman transport is strong. The effect of the approximation on oxygen was not estimated; it is assumed to be included in the noise.

\subsubsection{Anomaly Equations}

The noise in the mass conservation equations tends to dominate most tracer conservation equations. As a result, the original full property equations produce an ill-conditioned matrix (e.g., McDougall, 1989; Wijffels, 1993). To better condition the system, one can subtract the mass divergence times the average property concentration for each layer from each tracer conservation equation. As discussed by Wunsch (1996), the main issue, when using anomaly equations, is the determination of the degree of cancellation in the residual noise after subtraction.

We now estimate this degree of cancellation, or equivalently the size of the noise term in anomaly equations. The salt equation is taken for illustration. For a single zonal hydrographic section, and within a layer, the net transports of mass and salt are written

$$
\begin{aligned}
T_{M} & =\iint d x d z\left(\rho v(x, z)+n_{v}(x, z)\right) \\
\text { and } T_{S} & =\iint d x d z\left(\rho v(x, z) S(x, z)+n_{v s}(x, z)\right),
\end{aligned}
$$


where $v(x, z)$ is the meridional velocity; $\rho$ is density; the terms $n_{v}(x, z)$ and $n_{v s}(x, z)$ refer to noise in the advective transports of mass and salt at individual station pairs; $x$ is longitude and $z$ is the vertical coordinate. Let $\bar{S}$ be the mean salinity of the layer and $S^{\prime} \doteq S-\bar{S}$ the salinity anomaly. Then by subtraction $(1.12)-\bar{S} \times(1.11)$ one obtains an expression for the salinity anomaly transport:

$$
\begin{aligned}
T_{S^{\prime}} & =\iint \rho v(x, z) S^{\prime}(x, z) d x d z+\mathbf{n}_{S}^{\prime}, \\
\text { with } \mathbf{n}_{S}^{\prime} & \doteq \iint\left(n_{v s}(x, z)-\bar{S} n_{v}(x, z)\right) d x d z .
\end{aligned}
$$

A mass transport error $\mathbf{n}_{M}$ (the subscript $M$ stands for mass) is anticipated to produce a salt transport $\mathbf{n}_{S}$ of roughly $\bar{S} \times \mathbf{n}_{M}$ such that $\mathbf{n}_{S}^{\prime}$ should be much smaller than $\mathbf{n}_{S}$. We now formalize this statement. Assuming that the noise in the salt flux, $n_{v s}(x, z)$, is mainly due to noise in the mass flux $n_{v}(x, z)$ (as opposed to being mainly caused by variations in the salinity itself), $n_{v s}(x, z)$ may be written as

$$
\begin{aligned}
n_{v s}(x, z) & =n_{v}(x, z) \times S(x, z), \\
\text { or } n_{v s}(x, z) & =n_{v}(x, z) \times\left(\bar{S}+S^{\prime}(x, z)\right), \\
\text { so that } \mathbf{n}_{S}^{\prime} & =\iint n_{v}(x, z) S^{\prime}(x, z) d x d z .
\end{aligned}
$$

$\mathbf{n}_{S}^{\prime}$ may be divided into a component owing to the zonally averaged mass transport over the layer and a deviation that we call the horizontal eddy component:

$$
\begin{aligned}
\mathbf{n}_{S}^{\prime} & =\mathbf{n}_{M} \iint S^{\prime}(x, z) d x d z+\iint\left(n_{v}(x, z)-\mathbf{n}_{M}\right) S^{\prime} d x d z, \\
\text { with } \mathbf{n}_{M} & \doteq \iint n_{v}(x, z) d x d z .
\end{aligned}
$$

$\mathbf{n}_{M}$ is the noise in the zonally integrated mass flux over a layer. $\bar{S}$ is defined over a whole layer, possibly bounded by several sections, so that the first term of (1.18) (the zonal average) is in general non-zero. As an approximate estimate for the variance of the zonally averaged component, we chose to use $\left\langle\mathbf{n}_{M}^{2}\right\rangle\left\langle\Delta S^{2}\right\rangle$, where $\Delta S$ is the a priori size of salinity variations within the layer. The size of the horizontal eddy component is unknown a priori, and is assumed to be of the same magnitude or smaller than the zonally averaged component with possible correlation between the two. This speculative 
assumption is supported by studies of the heat (energy) flux variability (salt was used as an example, but similar anomaly equations are written for other tracers including internal energy). Jayne (1999) found that at all latitudes, variations in the baroclinic heat transport-which appears as a noise, $n_{0}$, in the heat equations-are about twice as large as variations in the horizontal eddy contribution to the heat transports. Instantaneously, salinity anomaly transports display a similar behavior as heat anomaly transport (e.g., Wunsch, 1996, Fig. 4-15) so that the noise in the salinity anomaly may be reasonably assumed to be similar.

Thus, the ad hoc, a priori, noise in the tracer anomaly equations for any tracer $C$ is

$$
\left\langle\mathbf{n}_{C}^{\prime 2}\right\rangle=4 \times\left\langle\mathbf{n}_{M}^{2}\right\rangle \times\left\langle\Delta C^{2}\right\rangle
$$

where $\Delta C$ is the standard deviation of tracer variations within the layer and the factor of 4 accounts for possible correlations between the zonally averaged and horizontal eddy components. The average tracer concentration $(\bar{C})$ in each layer was used in defining the anomaly equations. Expression (1.20) relies on the assumptions listed above. It is no more than a best guess, which turns out to produce consistent results (in terms of error bars) when used in the inversion. The main test of consistency comes from the fact that the residuals of all conservation equation are indistinguishable from zero within a posteriori error bars.

Wijffels (1993) weighted her anomaly equations in a similar way, but assumed that the salinity variations were dominated by vertical differences. Because we have no information on the noise in vertical exchanges, it is not clear whether this approach is more appropriate than ours. Nevertheless, vertical salinity differences are on average of the same order as the horizontal variations within a layer, but display more irregularities, which make them difficult to use as a reference.

\section{Silica and anomaly equations}

Anomaly equations are inappropriate for top-to-bottom silica conservation. Generally, most of the spurious mass residuals in layers occur close to the surface in part because of the larger variability in the thermal wind shear there. Because the average concentration 
over the water column $(\bar{C})$ is much higher than the concentration in the surface layers where the mass divergence actually occurs, the term $\bar{C} n_{M}$ (or $\bar{S}$ for salinity) of (1.14) is artificially large, creating an erroneous source or sink of silica in the top-to-bottom anomaly equation. Hence, using the surface concentration for creating the top-to-bottom anomaly equations or, for simplicity and consistency, using non-anomaly (original) equations for total silica conservation is more appropriate.

\section{Diagnostic of tracer divergences}

Tracer divergences were diagnosed following the anomaly formulation, effectively reducing the impact of noisy mass divergences from the tracer divergences. Doing so in each individual layer $i$ by using the average property $\vec{C}_{i}$ yields a corrected tracer divergence $\widetilde{\nabla \cdot C_{i}}$. But if $\bar{C}_{i}$ varies substantially with depth (as for temperature or nutrient anomaly), the corrected tracer divergence in the top-to-bottom integral $\left(\widetilde{\nabla \cdot C}_{T 2 B}\right)$ is not the same as the sum of the anomaly layer divergences:

$$
\widetilde{\nabla \cdot C}_{T 2 B} \neq \sum_{\text {layers }} \widetilde{\nabla \cdot C_{i}}
$$

Intuitively, using $\widehat{\nabla \cdot C_{i}}$ in individual layers is more reliable than the top-to-bottom corrected divergence because the mass divergences are removed more efficiently (see the discussion about silica just above). Still, if the difference (1.21) is significant within error bars, it will be interpreted as a warning that the a priori uncertainties (1.20) are inconsistent.

\subsection{The Gauss-Markov estimator}

The solution estimate $\tilde{\mathbf{b}}$ that satisfies (1.9) is obtained using the statistical Gauss-Markov method (i.e. Wunsch, 1996, page 184). We explicitly use the a priori knowledge of the size of the solution $\mathbf{b}, \mathbf{R}_{\mathbf{b}, \mathbf{b}} \doteq\left\langle\mathbf{b} \mathbf{b}^{T}\right\rangle$, and of the priori noise level variance, $\mathbf{R}_{\mathbf{n}, \mathbf{n}} \doteq\left\langle\mathbf{n} \mathbf{n}^{T}\right\rangle$ (the 〈〉 operator stands for the ensemble average). In the absence of a priori knowledge of the correlation between different equations and solution elements, $\mathbf{R}_{\mathbf{b}, \mathbf{b}}$ and $\mathbf{R}_{\mathbf{n}, \mathbf{n}}$ are taken to be diagonal. Determination of the a priori noise in the conservation equations is the focus of Chapter 2. The a priori size of the solution $\mathbf{b}$ is given in Section 2.4 of Chapter 2 
and for individual basins in Chapter 3 .

Given $\mathbf{R}_{b, b}$ and $\mathbf{R}_{\mathbf{n}, \mathbf{n}}$, the estimate $\overline{\mathbf{b}}$ that minimizes the deviation from the hypothetical true value is given by:

$$
\begin{aligned}
\tilde{\mathbf{b}} & =\mathbf{K} \mathbf{y} \\
\text { with } \mathbf{K} & =\mathbf{R}_{\mathbf{b}, \mathbf{b}} \mathbf{A}^{T^{\prime}}\left(\mathbf{A R}_{\mathbf{b}, \mathbf{b}} \mathbf{A}^{T}+\mathbf{R}_{\mathbf{n}, \mathbf{n}}\right)^{-1}
\end{aligned}
$$

If there is no bias $(\langle\tilde{\mathbf{b}}-\mathbf{b}\rangle=0)$, that is, both $\langle\mathbf{y}\rangle=0$ and $\langle\mathbf{n}\rangle=0,(1.22 \mathrm{a})$ is the Best Linear Unbiased Estimator (BLUE).

The error covariance is

$$
\begin{aligned}
\mathbf{P} & \doteq\left\langle\left(\tilde{\mathbf{b}}-\mathbf{b}_{\text {True }}\right)\left(\tilde{\mathbf{b}}-\mathbf{b}_{\text {True }}\right)^{T}\right\rangle \\
& =\mathbf{R}_{\mathbf{b}, \mathbf{b}}-\mathbf{K} \mathbf{A} \mathbf{R}_{\mathbf{b}, \mathbf{b}}
\end{aligned}
$$

The error estimate includes the part of the solution which is umresolved by the equations (the null-space). The residuals and uncertainties are computed from (1.9),

$$
\begin{aligned}
\tilde{\mathbf{n}} & =\mathbf{y}-\mathbf{A} \tilde{\mathbf{b}} \\
\mathbf{P}_{\mathbf{n n}} & =\mathbf{A} \mathbf{P} \mathbf{A}^{T}
\end{aligned}
$$

For consistency, it is checked that the residuals, after inversion, are indistinguishable from zero within error bars, which is a necessary condition for obtaining realistic error bars. (It follows that the residuals are within the a priori range $\mathbf{R}_{\mathbf{n}, \mathbf{n}}$ ).

Estimates for property transports $(\tilde{T})$ and uncertainties $(\delta \tilde{T})$ are derived from $(1.8)$ and $(1.22 a, b-1.23)$ :

$$
\begin{aligned}
\tilde{T} & =\Gamma_{r e l}+\mathbf{a}^{T} \tilde{\mathbf{b}} \\
\delta \tilde{T} & =\sqrt{\mathbf{a}^{T} \mathbf{P a}}
\end{aligned}
$$

where $\Gamma_{r e l}$ is again the property transport due to the relative velocity and $\mathbf{a}^{T}$ is a row vector containing the integrated property concentration at each station pair. 


\section{Chapter 2}

\section{The Error Budget of Inverse Box Models}

In this chapter I estimate the uncertainty in mass and silica transport estimates due to the non-synopticity of hydrographic sections (i.e., the departure of nearly-synoptic fields from true time-means), numerical errors in transport calculations and the measurement noise. In particular, a an eddy-permitting general circulation model (GCM) is used to estimate the extent to which a hydrographic cruise is representative of the true average density field and associated transports. This study is relatively extensive, and the reader may chose to go directly to the summary of a priori uncertainties that will be used to weight the conservation equations, Section 2.5 (page 62) and Section 2.6 (page 66).

\section{$2.1 \quad$ Introduction}

As we have seen, the linear inverse box model can be condensed into one equation:

$$
A b+\mathbf{n}=\mathbf{y},
$$

where $\mathbf{A}$ is a matrix, $\mathbf{b}$ is a vector containing all the unknowns of the system, $\mathbf{y}$ is the observation vector and $\mathbf{n}$ is the noise. Each row of (2.1) is an equation (or constraint) on $\mathbf{b}$ and, given some reasonable assumptions about the variance of $\mathbf{b}$ and $\mathbf{n}$, the inverse machinery provides an estimate for $\mathbf{b}$ and its covariance. In the present analysis, $\mathbf{b}$ 
contains the unknown reference velocities and the product $\mathbf{A b}$ represents the transport and transport divergence of different properties (mass, heat, nutrients...) due to the reference level velocities. $\mathbf{y}$ contains the transport from thermal wind, Ekman transport and other possible sources and sinks in the conservation equations. The expected values of the solution and of the noise are assumed to be identically null, that is, $\langle\mathbf{b}\rangle=\langle\mathbf{n}\rangle=0$.

The uncertainty in the solution $\mathbf{b}$ and in the associated fluxes depends upon: 1) the weight of the constraints (i.e., the a priori variance of $\mathbf{n}$, denoted $\left\langle\mathbf{n} \mathbf{n}^{T}\right\rangle$ ) and, 2) the a priori variance of $\mathbf{b}\left(\right.$ denoted $\left.\left\langle\mathbf{b b}^{T}\right\rangle\right)$. In most previous studies, these two a priori quantities were associated with the error in the net mass flux that could enter or leave an oceanic area (freshwater flux, mass storage). For instance, Macdonald (1995) and Rintoul and Wunsch (1991) required mass conservation using an a priori error of \pm 1 to $\pm 2 \mathrm{~Sv}$-an optimistic value, as will be suggested in this chapter.

In a related work, McIntosh and Rintoul (1997) tested the ability of an inverse box model to reproduce an instantaneous snapshot of the southern ocean circulation as computed with a quasi-eddy resolving general circulation model(GCM). They found the box model successful as long as the prior statistics on the solution variance (i.e., $\left\langle\mathbf{b} \mathbf{b}^{T}\right\rangle$ in our notations) were chosen to correspond to the true (GCM) solution. The object of this chapter is to review and quantify the different sources of error entering the conservation equations, and to provide estimates for the prior statistics $\left\langle\mathbf{n} \mathbf{n}^{T}\right\rangle$ and $\left\langle\mathbf{b} \mathbf{b}^{T}\right\rangle$.

\section{Mass Flux Estimation}

At a given time $t$, and assuming for the moment that geostrophy is a good approximation away from the surface layer, the mass transport through a transoceanic hydrographic section, $\mathrm{T}(t)$, is the sum

$$
\begin{aligned}
T(t) & =T_{R}(t)+T_{b}(t)+T_{E k}(t), \\
\text { with } T_{R}(t) & =-\iint d x d z \times \rho \frac{g}{\rho_{0} f} \frac{\partial \rho}{\partial x} \\
T_{b}(t) & =\iint d x d z \times \rho b(x) \\
\text { and } T_{E k}(t) & =-\int_{X_{W}}^{X_{E}} d x \times \rho \frac{\tau(t)^{x}}{f}
\end{aligned}
$$


$T_{R}(t)$ is the thermal wind transport relative to a chosen "initial zero-velocity-surface" (IZVS), or reference surface. $T_{b}(t)$ is the transport contribution from the reference velocity (the unknown) and $T_{E k}(t)$ is the near-surface Ekman transport. $\rho$ is density, $g$ is gravity, $\tau(t)^{x}$ the zonal wind stress; $f$ is the Coriolis parameter and $t$ is time.

The data I use span long time intervals: it takes one to three months to make a transoceanic section; and two different sections used in a box model may be separated by several years (Table 1.1, page 16). The object of the inverse model is to estimate the true time mean transports across all the sections involved. Write this average

$$
\bar{T}=\bar{T}_{R}+\bar{T}_{b}+\bar{T}_{E k}
$$

where the overbar denotes a time average. Because $\vec{T}_{R}$ is estimated from the measurements at a single time $t_{0}$, the existence of a solution that is mass conserving relies on the assumption that the large scale thermal wind transport at time $t_{0}, T_{R}\left(t_{0}\right)$, is representative of the time-average $\bar{T}_{R}$ to a good approximation.

It is useful to separate the measurement noise, $n^{\text {measurement }}$, and the model error, $n^{\text {model }}$. The measurement noise comes from uncertainties in the measurement of $T_{R}\left(t_{0}\right)$ (internal wave noise, eddy aliasing, bottom triangle, etc.) while the (inverse) model error represents the limitation to a barotropic adjustment, permitting no change in the vertical shear-thus in $T_{R}$. It follows that the model error corresponds to the difference between $T_{R}\left(t_{0}\right)$ at the time of each section, and the time-average $\bar{T}_{R}$. This relationship may be written by

$$
\begin{aligned}
T_{R}^{\text {true }}\left(t_{0}\right) & =T_{R}^{\text {measured }}\left(t_{0}\right)+n^{\text {measurement }} \\
\bar{T}_{R} & =T_{R}^{\text {true }}\left(t_{0}\right)+n^{\text {model }}
\end{aligned}
$$

As we will see, the error from the oceanic baroclinic variability, $n^{\text {model }}$, dominates the total error. [In a more elaborate inverse model like the ones of Mercier (1986) or Wunsch (1994), change is allowed in the density field, thus reducing the model error. Because no such change is allowed here, any noise in $T_{R}$ may lead to a net mass imbalance in the solution.]

The sizes of $n^{\text {model }}$ and $n^{\text {measurement }}$ are estimated at three latitudes in the Atlantic: $24^{\circ} \mathrm{N}, 36^{\circ} \mathrm{N}$ and $48^{\circ} \mathrm{N} ; n^{\text {model }}$ is estimated with the help of a near-eddy-resolving general 
circulation model (GCM), while the measurement errors are estimated from data and experiments. The model error is investigated in Section 2.2. There it is shown that the oceanic response to Ekman transport fluctuations is essentially a balance between $T_{E k}(t)$ and $T_{b}(t)$ (Section 2.2.2) so that the variability in the relative transport is relatively small (but not negligible, Section 2.2.3). Along with the error budget in the mass flux, the model error in the silica equations is explored in Section 2.2.4. Measurement errors are quantified in Section 2.3; those include internal wave noise (Section 2.3.1); geostrophy (Section 2.3.2); bottom triangle approximation (Section 2.3.3) and mean Ekman transport (Section 2.3.4). The reference velocity variance in the GCM is quantified in Section 2.4. In Section 2.5, all components of the uncertainty are summarized and discussed. The application to our global hydrographic inverse model is described in Section 2.6.

\subsection{Model Error}

\subsubsection{The general circulation model (GCM)}

The GCM that I used to test the representativity of one-time hydrographic sections is a $\frac{1}{4}^{\circ}$ lateral resolution global circulation model run at the US Naval Post Graduate School (see Semtner and Chervin, 1992; Stammer et al., 1996). The model has 20 vertical levels and was forced with the daily wind stress fields from the European Center for Medium range Weather Forecast (ECMWF). The surface temperature and salinity were restored to the monthly fields of Levitus et al. (1994) with a 30-day time scale [see Stammer et al. (1996) for more details]. Stammer et al. (1996) compared the model output with both altimetric and hydrographic observations and found that the GCM reproduced successfully the major features of the large scale oceanic circulation and the structure of the variability. However, the GCM underestimated the amplitude of the variability by at least factor of 2 to 4 on most scales. The discrepancy was highest in the mesoscale range. The model run, labeled POCM 4-B, was extracted by S. Jayne (1999) and made available to me for the purpose of this study. Temperature, salinity, velocity and sea surface height data were subsampled every third day for a period of 8 years (1987 to 1994) and at three latitudes corresponding to actual hydrographic data across the Atlantic: $24^{\circ} \mathrm{N}, 36^{\circ} \mathrm{N}$, and $48^{\circ} \mathrm{N}$. 


\subsubsection{Oceanic response to Ekman fluctuations}

Areal integration of the GCM sea surface height between $36^{\circ} \mathrm{N}$ and $48^{\circ} \mathrm{N}$ revealed that in the GCM, the rate of change in mass storage did not exceed $\pm 0.2 \mathrm{~Sv}$ between the two latitudes. Similarly, altimetric observations of the ocean do not show large variations in the mean sea level (Wunsch, personal communication, 1997), suggesting that the Ekman mass inflow is almost instantaneously compensated by an interior flow in the opposite direction (e.g., Jayne, 1999). (On seasonal time scales there are variations from steric height change, though.)

Böning and Hermann (1994) studied the structure of the compensating interior flow and found it depth-independent, at mid-latitudes in a near eddy-resolving model of the North and Equatorial Atlantic. An integration of the present GCM transport in different layers confirmed that the temporal variation in the shear was smaller and slower than the depth-independent part. This barotropic response suggests that the thermal wind is stable at mid-latitudes, as will be confirmed in Section 2.2.3: most of the fluctuations in the Ekman transport are represented by $T_{b}(t)$ (Eq.2.2). At low latitudes, baroclinic waves can propagate faster than at mid and high latitudes, and the oceanic response is different (Böning and Herrmann, 1994). The study of the low latitude response to variable wind forcing is postponed to a possible extension of this work.

The Ekman transport was computed across each section for one year in the Atlantic Ocean from the ECMWF wind stress fields (Fig.2-1, thick line) using the classical Ekman formula (2.5). The Ekman transports change quickly with variations of up to $30 \mathrm{~Sv}$ in a few days at all latitudes. (The accuracy of the ECMWF wind stress themselves are discussed later in Section 2.3.4.) The same winds were used to compute the Ekman transports as were used to force the GCM.

On Figure 2-1 is overlain the Ekman transport and the total flow beneath $100 \mathrm{~m}$ in the GCM. The balance between the two curves is not exact because of energetic inertial oscillations. But if high frequencies are filtered, the imbalances do not exceed $\pm 0.8 \mathrm{~Sv} \mathrm{rms}$ (not shown), showing that Eq.(2.5) is appropriate to use in the GCM. (Indeed, the numerical parameterization of momentum transfers in the GCM upper layers is close to Eq.2.5.) The immediate consequence of the oscillations is a large fluctuation in the energy flux due 

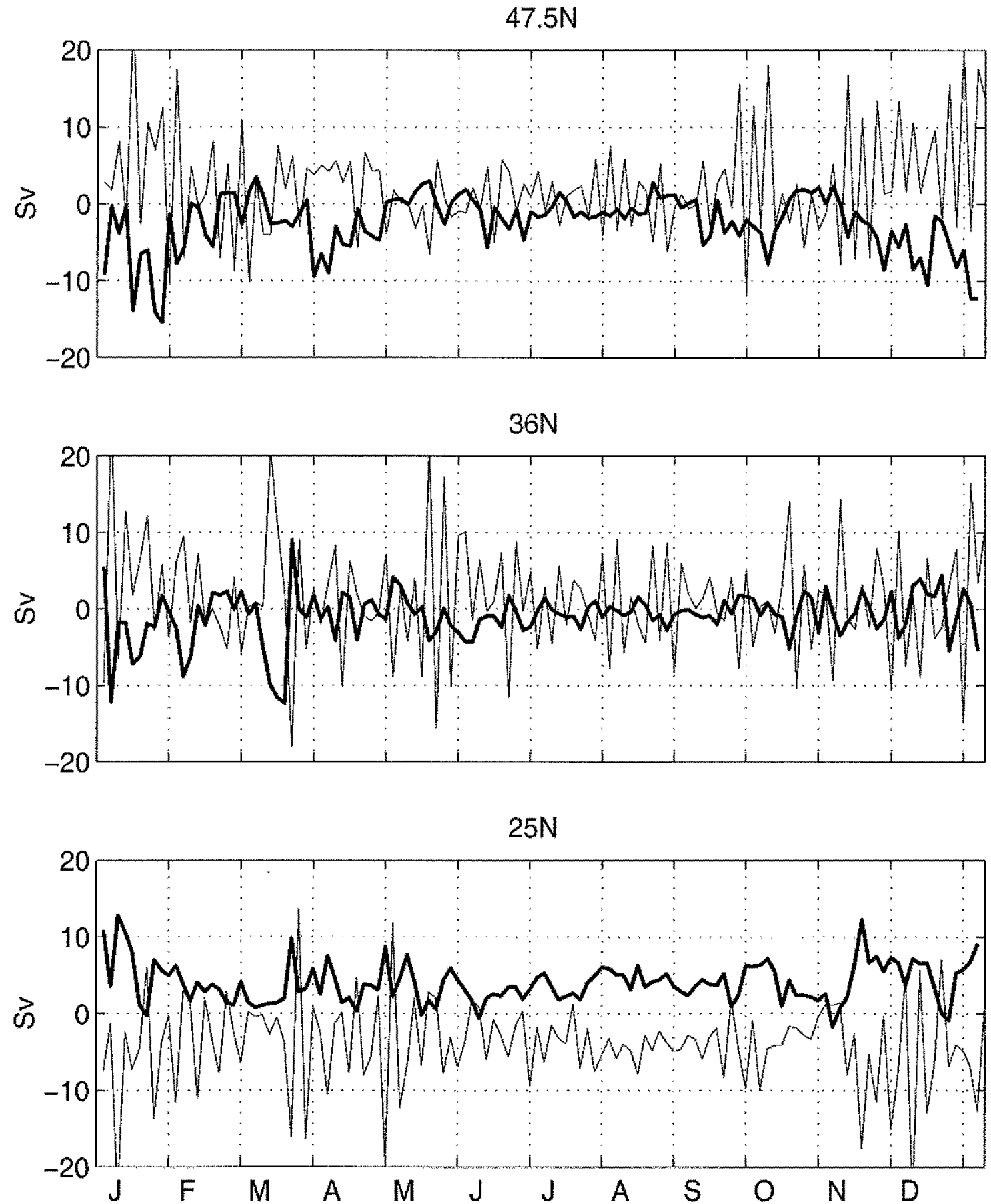

Figure 2-1: Time series of the Ekman flow (thick line) and the integrated model flow beneath $100 \mathrm{~m}$ (thin line) across the Atlantic at $47.5^{\circ} \mathrm{N}, 36^{\circ} \mathrm{N}$ and $24^{\circ} \mathrm{N}$. High frequency inertial oscillations are part of the model response. The two curves nearly cancel each other at low frequencies (not shown). 
to the temperature contrast between the Ekman layer and the average temperature over the water column (Jayne, 1999).

Because the Ekman transport fluctuations are almost balanced by a barotropic return flow, different hydrographic sections can be consistently combined in an inverse box model as long as the time-mean Ekman transport is used for all sections. Then, the calculated energy flux is representative of the time-average energy flux.

\subsubsection{Baroclinic variability}

I now turn to the error caused by baroclinic variability, that is, the deviations between the instantaneous transport $T_{R}(t)$ and the average $\overline{T_{R}}$. Locally, mesoscale eddies introduce important deviations from the mean transport, so that estimating the time-average thermal wind current would require an enormous data set (would be inappropriate to represent small-scale property transports). However, eddy effects tend to cancel out when integrated on large scales so that the zonally integrated thermal wind transport is relatively stable.

The baroclinic variability in the ocean is a broad subject. In their theoretical study of the seasonal variability, Gill and Niller (1973) found that the ocean response (baroclinic and "barotropic") to seasonal forcing was relatively small away from the Equator. Air-sea exchanges of heat are stored locally and changes in the horizontal velocities are weak. Even on seasonal time scales, the authors found that most of the response is barotropic (i.e., independent of the density structure) and moreover, that horizontal advection of temperature and salinity by barotropic currents does not produce significant baroclinic variations. But although barotropic current variations are locally small $\left( \pm 3 \mathrm{~mm} \mathrm{~s}^{-1}\right)$, they produce transports of $\pm 30 \mathrm{~Sv}$ when integrated on large scales.

Variability in the upper North Atlantic on longer time scales has been investigated by several authors, including Reverdin et al. (1997) and Curry et al. (1998) using the few available long time series of temperature and salinity. Large variations occur in the temperature and salinity field on 10 year time-scales. Salinity anomalies propagate in the North Atlantic and influence the convection regimes and in turn, the production of deep water masses (Reverdin et al., 1997). For instance, the Labrador Sea Water outflow is, for a similar density, alternatively cold and fresh or warm and salty. In turn, changes in the 
surface conditions are imprinted in the deep waters, and the corresponding signals can be observed in the deep Bermuda waters (Curry et al., 1998). The variability relates to the North Atlantic Oscillation index and sea ice coverage (i.e., Reverdin et al., 1997; Curry et al., 1998).

Based on a simple, baroclinic, linear Rossby-wave model, Sturges et al. (1998) report decadal variations in the zonal differences of sea surface height of $\pm 4 \mathrm{~cm}$ in the Atlantic at $34^{\circ} \mathrm{N}$ and $\pm 10 \mathrm{~cm}$ at $18^{\circ} \mathrm{N}$ (away from the Gulf Stream). Assuming these variations were confined within the surface $1000 \mathrm{~m}$, the baroclinic variation in the gyre strength would be respectively $\pm 5 \mathrm{~Sv}$ at $34^{\circ} \mathrm{N}$ and $\pm 20 \mathrm{~Sv}$ at $18^{\circ} \mathrm{N}$. These variations are entirely due to changes in the wind stress curl, and their model produced a reasonable agreement with hydrographic data. However, the variations in the net, coast-to-coast flux, are probably much smaller than the ones they reported because the western boundary current, which they did not include, probably balances the variations in the gyre strength.

Greatbach et al. (1991) obtained variations of similar amplitude -but with the opposite sign-using a linear diagnostic model of the North Atlantic based on climatological wind and density data. Comparison of the mean circulation for the period 1955-1959 with that of 1970-1974 showed a $30 \mathrm{~Sv}$ decrease in the Gulf Stream gyre. Differences in the sampling between the two pentads are a large source of uncertainty, however, half of the variations were explained by changes in the density field above $1500 \mathrm{~m}$ following a weakening of the subtropical gyre. The other half is uncertain because of the large time and space sampling differences in the deep waters between the two time periods considered. In other oceanic regions, similar variations may be associated with ENSO and other large scale atmospheric patterns.

Repeat hydrographic sections are appropriate to study the long term interannual variability in the net fluxes. At $24^{\circ} \mathrm{N}$ and $36^{\circ} \mathrm{N}$, sections were done during IGY (1957-1959), then in 1981 and recently in 1992-1993. Evidence of time shifts in the water mass characteristics have been found (e.g., Bryden et al., 1996, Arbic and Owens, 1999). Roemmich and Wunsch (1985) found the geostrophic transports (thermal-wind+barotropic adjustment) from the two first occupations to be qualitatively similar, but the vertical profiles of meridional transport exhibited noticeable differences in their structure and water mass 
fluxes. Their differences are compared with my results at the end of this section.

In this section, I use the GCM output to quantify the thermal-wind variability. To remove the large variability in the GCM associated with inertial oscillations (to first order unrelated to the thermal wind, Section 2.3.2), all calculations were based on the absolute geostrophic velocities from the pressure field. That is, from the thermal wind referenced at the surface and the sea surface elevation $(\eta)$ :

$$
v_{g a}(x, z)=\frac{g}{f} \frac{\partial \eta(x)}{\partial x}-\frac{g}{\rho_{0} f} \int_{0}^{z} \frac{\partial \rho(x, z)}{\partial x} d z
$$

where $x$ is the zonal distance, $z$ the depth, $g$ the gravity, $f$ the Coriolis parameter and $\rho$ the density. The Rossby number in the model did not exceed $\varepsilon=0.2$. Near the Gulf-Stream the effect of horizontal friction in the GCM is relatively small so that, away from the Ekman layer and except for inertial oscillations, geostrophy is expected to first order. Still, a large ageostrophic transport was found in the GCM output. It occurred on the grid scale near the Gulf Stream, but neither the non-linear terms nor the friction was able to support the $5 \mathrm{~Sv}$ deviation observed (50\% of the local transport). Several experiments led to the conclusion that the ageostrophy was due to a numerical artifact, possibly the bottom drag or an implicit diffusion acting on small scales (Appendix A). As the ageostrophic flow is depth-independent the present analysis of baroclinic variability was not directly affected.

Because the GCM was integrated for 8 years (1987 to 1994) but forced seasonally with climatological temperature, salinity and heat fluxes (Stammer et al., 1996), the only interannual patterns come from the variations in the wind stress. Because of the repetitive seasonal forcing, the variability in the GCM thermohaline circulation is underestimated. Because the GCM underpredicts the oceanic variability (page 30), the variability over those 8 years provides a lower bound on the actual variability. (On the other hand, model drift, which was not removed, introduces additional spurious variability.)

I start by investigating the seasonal and interannual variability in $T_{R}$ assuming all hydrographic sections are measured instantaneously. Then, a one-month-long section is simulated in the GCM to assess the effect of the finite time required to sample the ocean. 


\section{Instantaneous section}

Following the dynamic method, the velocity was split into a reference velocity (denoted hereafter RV or $b(x))$ plus a relative velocity $v_{R}(x, z)$ so that

$$
v_{g a}(x, z)=v_{R}(x, z)+b(x)
$$

The velocity is referenced to a deep isopycnal surface, as in the hydrographic model of Macdonald (1995): $\sigma_{4}=45.81$ at $25^{\circ} \mathrm{N}$ and $36^{\circ} \mathrm{N}$, and $\sigma_{2}=36.87$ at $48^{\circ} \mathrm{N}$. At $25^{\circ} \mathrm{N}$ and $36^{\circ} \mathrm{N}$ the reference isopycnals are about $3000 \mathrm{~m}$ deep, while at $48^{\circ} \mathrm{N}$ the reference surface lies between 1500 and $2000 \mathrm{~m}$.

The absolute and relative geostrophic transports were integrated in individual layers similar to the ones used by previous authors (Macdonald, 1995; Rintoul and Wunsch,1991 ; Roemmich and Wunsch, 1985) and listed in Table 2.1. However, for simplicity, interface depths were computed without allowing changes in the reference pressure-in contrast to Macdonald's definition ${ }^{1}$. Neutral surfaces (Jackett and McDougall, 1997, McDougall, 1987) are more consistent with the Macdonald definition, but the code for computing neutral surfaces is too slow for computation at each time step. A snapshot of the interface positions is shown on Figure $2-2$ at $36^{\circ} \mathrm{N}$.

The integration was done every third day for eight years. Figure 2-3 shows the total transport $\left(T_{R}+T_{b}\right)$ across latitudes $25^{\circ} \mathrm{N}, 36^{\circ} \mathrm{N}$ and $48^{\circ} \mathrm{N}$ along with the contribution

\footnotetext{
${ }^{1}$ In the Macdonald definition a reference pressure is assigned to an isopycnal at one end of a hydrographic section. The isopycnal position is computed along the section. If the isopycnal deviates too much from the original reference, the local reference pressure is changed to continue the calculation along the section..
}

\begin{tabular}{|l|l|l|}
\hline$\sigma_{0}$ & $\sigma_{2}$ & $\sigma_{4}$ \\
\hline 26.4 & 36.87 & 45.81 \\
\hline 26.8 & 36.94 & 45.85 \\
\hline 27.10 & 36.98 & 45.87 \\
\hline 27.3 & 37.02 & 45.895 \\
\hline 27.5 & & 45.91 \\
\hline 27.7 & & 45.925 \\
\hline
\end{tabular}

Table 2.1: Potential density interfaces. Reference is taken at the surface, $2000 \mathrm{db}$ and $4000 \mathrm{db}$. 


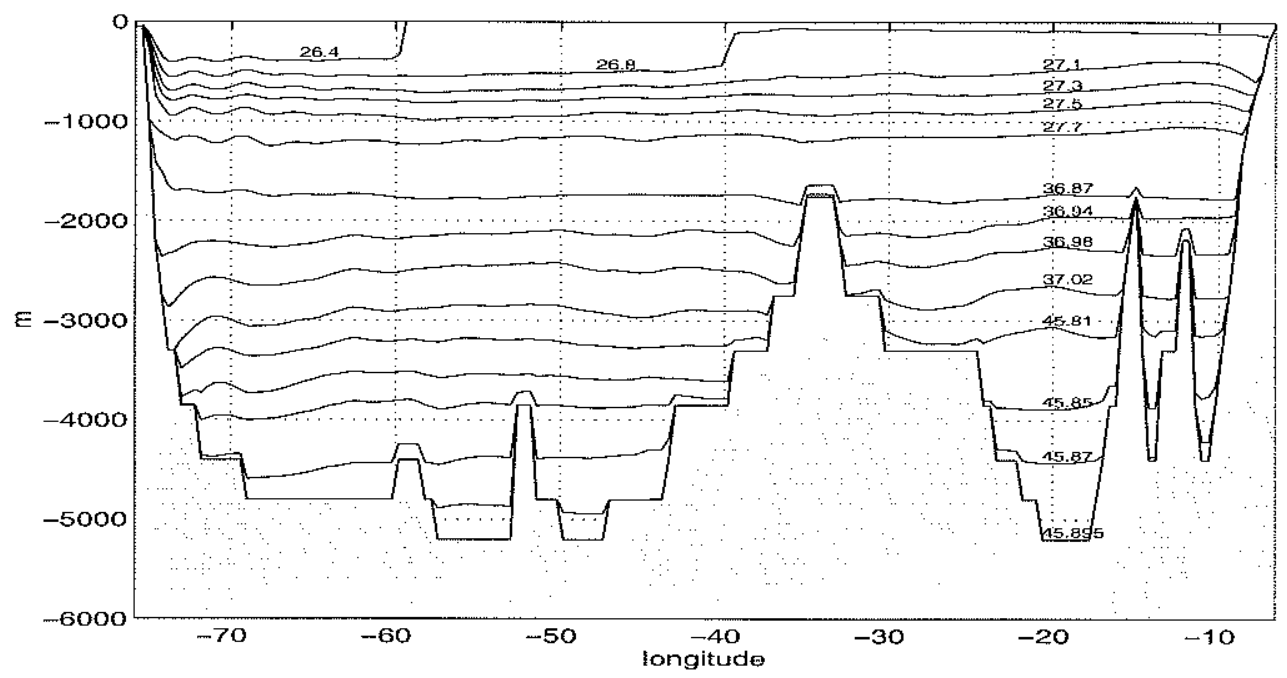

Figure 2-2: Isopycnal interface position along section $\mathrm{A} 36^{\circ} \mathrm{N}$

from the relative velocity $\left(T_{R}\right)$. The layer thicknesses and depths plotted correspond to an average across the section. Note the northward transports are displayed in each individual layer in contrast to the transport streamfunction that is used in the remainder of this thesis.

In the deep layers, most of the variability in the total transport $\left(T_{R}+T_{b}\right.$, Fig. 2-3, shaded areas) may be ascribed to the depth-independent contribution ( $T_{b}$, not shown) while a small fraction ( 10 to $30 \%$ across $25^{\circ} \mathrm{N}$ and $36^{\circ} \mathrm{N}$ ) is reflected in the relative transport $T_{R}$. On the other hand, the relative transport variability dominates in the surface layers. The variability in the thermal wind transport, $T_{R}$, changes with latitude but generally it does not exceed $\pm 0.5 \mathrm{~Sv}$ below $2000 \mathrm{~m}$ while it increases from $\pm 1 \mathrm{~Sv}$ to $\pm 2 \mathrm{~Sv}$ above $2000 \mathrm{~m}$ (all numbers are the one standard deviation variability). Variability in the deep layers is higher at $48^{\circ} \mathrm{N}$ in the $2000-4000 \mathrm{~m}$ range, possibly because the deep western boundary current variations are stronger there. The variability in the total relative transport (numbers below each plot) is relatively large: $\pm 6.2 \mathrm{~Sv} \operatorname{rms}\left(25^{\circ} \mathrm{N}\right), \pm 5 \mathrm{~Sv} \operatorname{rms}\left(36^{\circ} \mathrm{N}\right)$ and $\pm 2.5 \mathrm{~Sv} r m s\left(48^{\circ} \mathrm{N}\right)$. On short time scales, such deviations are in balance with a barotropic (or Ekman) return flow. However, those variations must be taken into account for combining consistently different sections, as discussed later (page 64). 

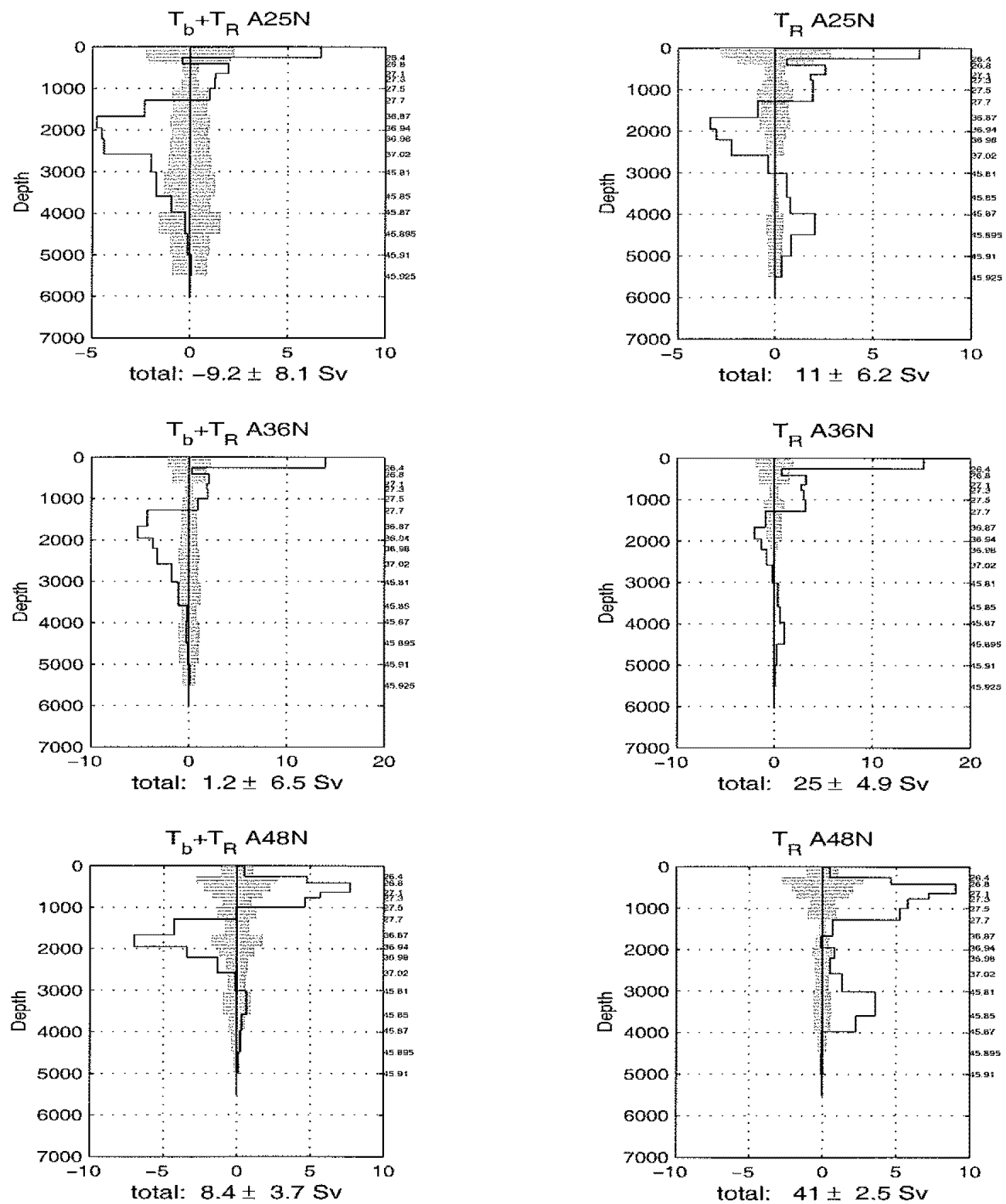

Figure 2-3: Individual layer mass transports across $25^{\circ} \mathrm{N}$ (upper), $36^{\circ} \mathrm{N}$ (middle) and $48^{\circ} \mathrm{N}$ (lower) in the GCM. The plots on the left give the total transport while the plots on the right give the thermal wind contribution. The eight year average is indicated by the lines while the variability is given by the shaded area (one standard deviation). 
In principle, in the real ocean, the net transport across each section (numbers below the graphs on the left) is equal and opposite to the average Ekman transport; but because of the ageostrophy in the GCM, this balance is not achieved by the time-mean geostrophic flux, producing an error of order $10 \mathrm{~Sv}$ at $36^{\circ} \mathrm{N}$.

For comparison, similar calculations were done over a single year (not shown). The variability in $T_{R}$ is smaller by a factor of 2 in the deep layers while it is similar in the surface layers and for the total flux.

As mentioned above, the variability may be underestimated by a factor two or four, according to the GCM evaluation of Stammer et al. (1996). Roemmich and Wunsch (1985) compared the geostrophic flux through repeat sections at $24^{\circ} \mathrm{N}$ and $36^{\circ} \mathrm{N}$ taken about 25 years apart (1957-1981). They found deviations of order $1 \mathrm{~Sv}$ in individual isopycnal layers (the same layers as I used) and up to $2 \mathrm{~Sv}$ near the surface. Those errors were found to be systematic over several layers, leading to relatively large deviations in water mass transports: up to $7 \mathrm{~Sv}$. The authors pointed out that part of the deviation may come from actual oceanic variability while the other part may be an enhancement of the variability by the simple model used, i.e., a uniform correction to the reference velocity. Direct comparison with the GCM results is somewhat difficult because different reference surfaces were used and because the Roemmich and Wunsch transports are already adjusted for mass conservation over the two sections so that their reported variability includes a barotropic adjustment. Still, the magnitude of the variations is consistent with those derived from the GCM.

At $24^{\circ} \mathrm{N}$, Lavin (1993) did a similar comparison including the more recent (1992) section at $24^{\circ} \mathrm{N}$. The top-to-bottom geostrophic transports on the three occupations from Bahamas to Africa referenced to $1100 \mathrm{~m}$ were $-41.0 \mathrm{~Sv}(1957),-31.7 \mathrm{~Sv}(1981)$ and $-36.9 \mathrm{~Sv}$ (1992). Assuming a constant transport in the Florida Strait (e.g., Schott et al., 1988b) the geostrophic variations range between $+9.3 \mathrm{~Sv}(1957 \longrightarrow 1981)$ and $-5.2 \mathrm{~Sv}(1981 \longrightarrow 1992)$. Both deviations are consistent with our $\pm 6 \mathrm{~Sv}$ GCM variability in the total flux at $24^{\circ} \mathrm{N}$.

At $24^{\circ} \mathrm{N}, 36^{\circ} \mathrm{N}$, and $48^{\circ} \mathrm{N}$, Koltermann et al. (1999) compared the transports from repeat sections occupied since the IGY through recent intensive WOCE surveys. Based on the assumption of Sverdrup balance, and after adjustment to the initial velocity field, 
the meridional overturning in the interval 1957 to 1997 varied between 8 and $21 \mathrm{~Sv}$ at $36^{\circ} \mathrm{N}$ and between 10 and $20 \mathrm{~Sv}$ at $48^{\circ} \mathrm{N}$. This variability is larger than the one inferred from the GCM. However, not only does it include part of the barotropic variability after correction, but it also relies upon the Sverdrup relation, which is inappropriate in the sub-polar North Atlantic Ocean (Luyten et al., 1985). As a result, it is not possible to distinguish the part of this variability which is due to changes in the density field alone, which I explored in the GCM. Also, at $48^{\circ} \mathrm{N}$, measurement errors are enhanced because the deep western boundary current flows against the continental shelf (see below, Section 2.3.3).

At $48^{\circ} \mathrm{N}$ in Koltermann et al. (1999) and the GCM, a shallow reference surface was used. In the presence of oceanic eddies, such a choice creates large velocities at the bottom, reflecting the large eddy velocities at the thermocline depth. Above rough topography, these eddy-related bottom transports do not cancel out, creating an artificially large variability in the transport. Hence, starting with a deep reference surface is more likely to minimize this error, as long as enough range of adjustment is allowed in the reference velocitics.

On the whole, the GCM variability at $24^{\circ} \mathrm{N}, 36^{\circ} \mathrm{N}$ and $48^{\circ} \mathrm{N}$ is in agreement, within a factor of two or smaller, with the inferences from repeat sections.

\section{Eddy aliasing}

Another problem that arises while sampling a constantly varying ocean is aliasing. Two sorts of alias take place: 1) eddy "Doppler" aliasing and 2) spatial aliasing. The eddy "Doppler" appears because it takes one month for a ship to cross the Atlantic, so that the field "seen" by the ship is different from any instantaneous field. An extreme example would be a ship moving at the same speed as an eddy: the T,S measurements would show flat isopycnals over the section. In a more realistic situation, eddies moving with the ship exhibit smoother gradients, while eddies moving against the ship exhibit steeper gradients, with possible effects on property transports.

To explore the effect of this possible alias on the mass flux, cruises were simulated along $36^{\circ} \mathrm{N}$ in the GCM so that the outputs were sampled in one month across the Atlantic. The mass flux was then compared with the annual average flux obtained from synoptic model- 
data. One cruise was simulated for each month of 1993. The layer transports did not show departures from the 1993 average that would exceed the natural variability reported in the preceding section. Still, the effect of aliasing is visible in the top-to-bottom thermal wind transport: $T_{R}$ varied by $\pm 4.8 \mathrm{~Sv}$ rms between the 12 cruises while the variability from instantaneous sections at $36^{\circ} \mathrm{N}$ from 3 -day snapshots and for one year is $\pm 3.5 \mathrm{~Sv} \mathrm{rms}$. The difference, $40 \%$ of the natural variability, does not exceed the $\pm 5 \mathrm{~Sv}$ rms interannual variability there and for this "order of magnitude" error budget, the ship aliasing effect will be neglected with respect to the natural variability. Because at mid-latitudes a research ship crosses the Atlantic about 100 times faster than the first baroclinic Rossby wave (assuming it takes 10 years for a baroclinic Rossby wave to cross the ocean), aliasing is unlikely to introduce a large error.

The second possible error, spatial aliasing, is caused by finite station spacing, which is generally close to the first Rossby radius. Because much of the eddy energy occurs on scales comparable to the first Rossby radius, the data may alias the scales that are below the station spacing into large scales. Unfortunately, the GCM output is inadequate to quantify this error because subsampling the GCM fields would incur errors from the representation of the bottom topography. Roemmich and Wunsch (1985) compared the transports between two coarse-resolution and two fine-resolution sections taken at different times in the North Atlantic. They concluded that temporal variability dominated the changes in geostrophic transports. Still, it is not certain that modern sections have enough

resolution. A possible test would be to sub-sample a high resolution XBT line to study the effect on property transports. To my knowledge, no such study has been done and I have to assume that the spatial aliasing error is not large compared to the other errors.

\subsubsection{Silica Variability}

Silica is a useful constraint in inverse box models because to a good approximation it is believed to be conserved in the ocean (e.g. Section 4.3.1; Robbins and Bryden, 1993; Robbins and Toole, 1997; Rintoul and Wunsch, 1991; Wunsch et al., 1983). Like most nutrients, the dissolved silica concentration is depleted in shallow waters. Consequently, the silica flux does not behave like the mass or energy flux. As for the mass conservation 
budget, the silica variability introduces a noise $n_{S i}^{\text {model }}$ in the silica conservation equations. In this section, I provide an estimate for the silica flux variability for each layer in the Atlantic at $36^{\circ} \mathrm{N}$. The variability in the meridional flux of silica is impossible to estimate from existing observations and the GCM does not contain any nutrient information. To remedy this situation, an artificial silica variable was created in the GCM based on a simple relation between potential temperature, longitude and silica. I first constructed this relation, based on a hydrographic section at $36^{\circ} \mathrm{N}$, then, I implemented it in the GCM output fields to obtain the variability in the advective silica flux.

Figure 2-4 shows the silica concentration versus temperature in the Atlantic at $36^{\circ} \mathrm{N}$. An analytical function was sought in the form of Chebyshev polynomials tapered with an exponential to remove the dominant vertical structure:

$$
S_{i}(t, l)=\sum_{i=0}^{6} a_{i} \times T_{i}(\theta) \times e^{-10 \times(\theta-1)}+a_{7} \times l
$$

where $S_{i}$ is the silica concentration, $l, \theta$ are the longitude and the temperature scaled to the interval $[-1,1] . T_{i}(t)$ is the $i$ th Chebyshev polynomial (type 1) and the cocfficients $a_{i}$ are obtained by regression. The regression was done independently for each side of the midAtlantic ridge as the silica pattern varies significantly. The regression curves are overlain on Figure 2-4. Polynomials of degree 6 were sufficient to represent approximately the behavior of silica with temperature. Residuals are shown on Figure 2-5. The rms residuals do not exceed $2 \mu \mathrm{mol} / \mathrm{kg}$, which is much lower than the average silica concentration.

Using the analytical representation, the silica field was computed from the GCM output every third day for eight years. Figure 2-7 compares the observed silica from the hydrographic section (upper panel) with a snapshot of the "GCM silica" (lower panel). The GCM silica is smoother than the observations because of the smoother temperature field. The step above the mid-Atlantic ridge arises from the change in the regression function. This unrealistic feature did not introduce significant spurious variability.

The geostrophic flux of silica was obtained, as for the volume flux, every third day through each of the isopycnal layers defined in Section 2.2.3. Figure 2-6 shows the total mean flux and variability $\left(T_{R}+T_{b}\right.$, left plot) and the thermal wind contribution $\left(T_{R}\right.$, right plot). Because of the increase in the silica concentration with depth, the variability 


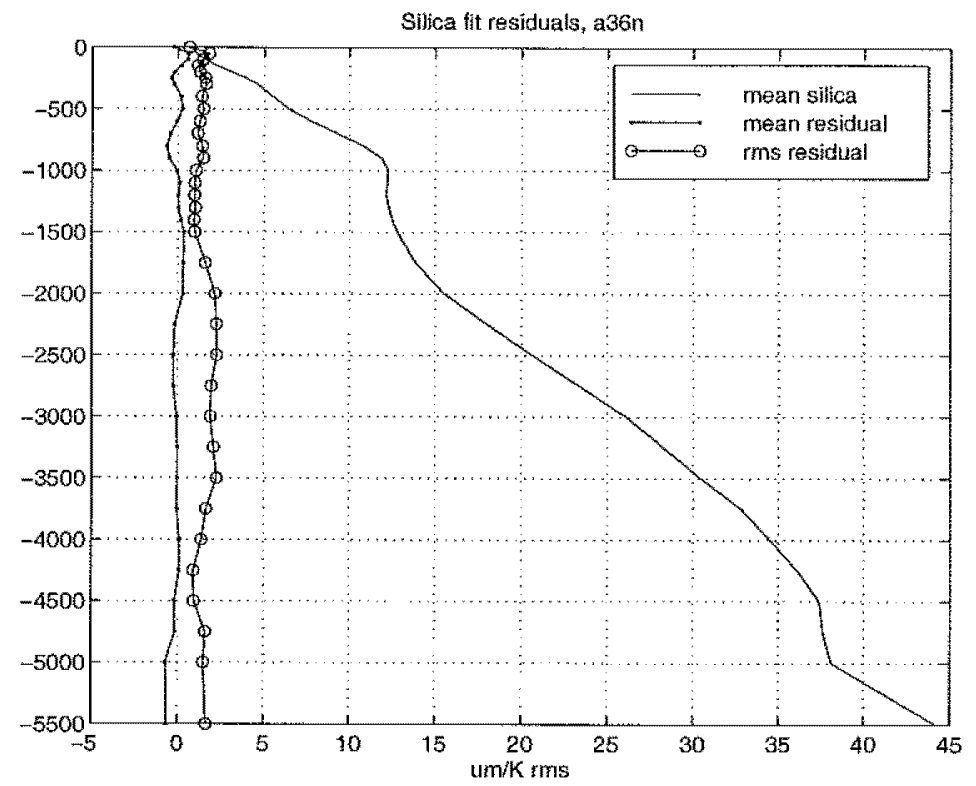

Figure 2-5: Silica residual after regression, at each depth. For comparison, the mean silica concentration is overlain. Silica is in $\mu \mathrm{mol} \mathrm{kg}^{-1}$ while depth is in decibar. (The accuracy of very best routine measurements of silica is $0.5-1.0 \mu \mathrm{mol} \mathrm{kg}{ }^{-1}$ ). 


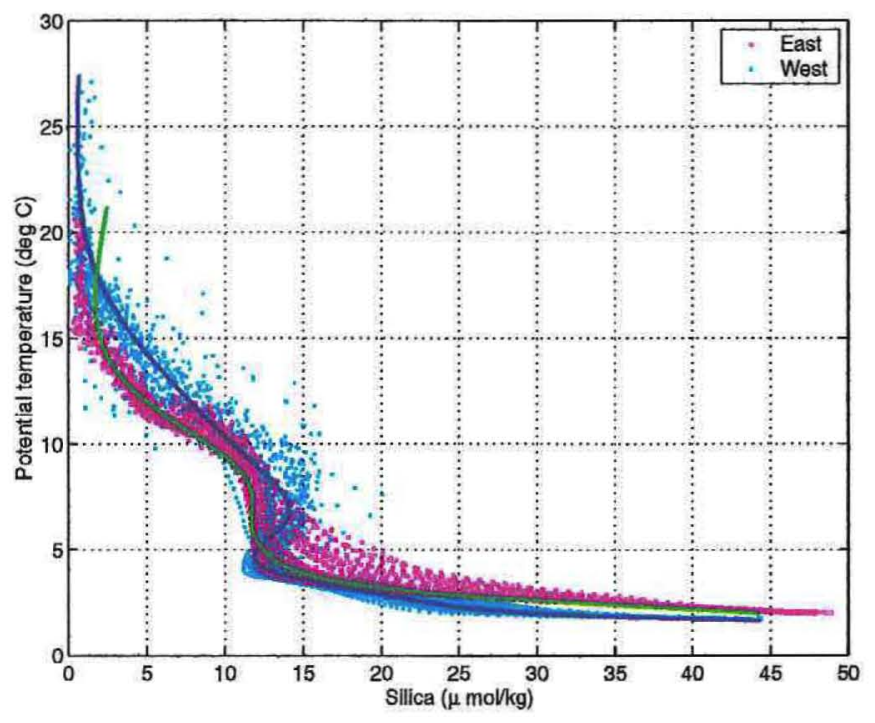

Figure 2-4: Silica versus potential temperature at $36^{\circ} \mathrm{N}$ in the Atlantic. Data east of the mid-Atlantic ridge are represented by purple open circles while on the west they are represented by the blue dots. The two curves (respectively green and blue) correspond to regressions obtained on either side of the mid-Atlantic ridge.

in the total flux now increases with depth, in contrast with the mass flux variability. However, the variability due to the thermal wind, $\left\langle\left(n_{S i}^{\text {model }}\right)^{2}\right\rangle$, is relatively constant with $\pm 10 \mathrm{kmol} \mathrm{Si} \mathrm{s}^{-1}$ in each layer. The variability in the net flux from thermal wind is about $\pm 50 \mathrm{kmol} \mathrm{Si} \mathrm{s}^{-1}$. While I believe that my results give a lower bound on the actual oceanic variability, the mean GCM silica fluxes (Fig. 2-6, lines) are probably unrealistic because 1) of the ageostrophic issue discussed above and 2) the re-created silica field is not necessarily compatible with the average thermohaline circulation. Silica was chosen among other nutrients because of its relatively simple biogeochemistry, but the results are adaptable to other nutrients that have approximately the same vertical structure. 

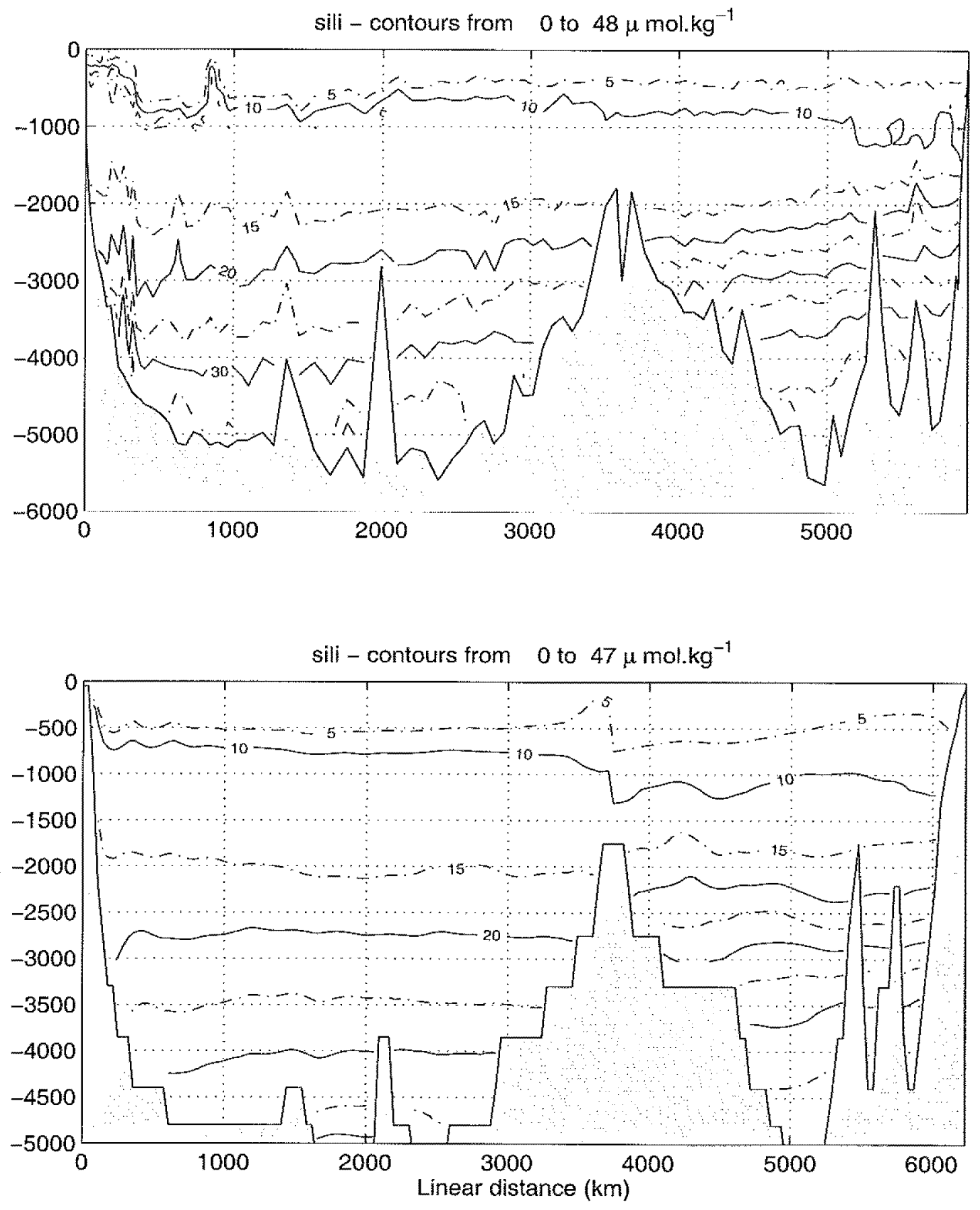

Figure 2-7: (Upper) Silica observed from the $36^{\circ} \mathrm{N}$ hydrographic section. The snapshot of the GCM reconstructed silica (Lower) shows a similar, but smoother field. 

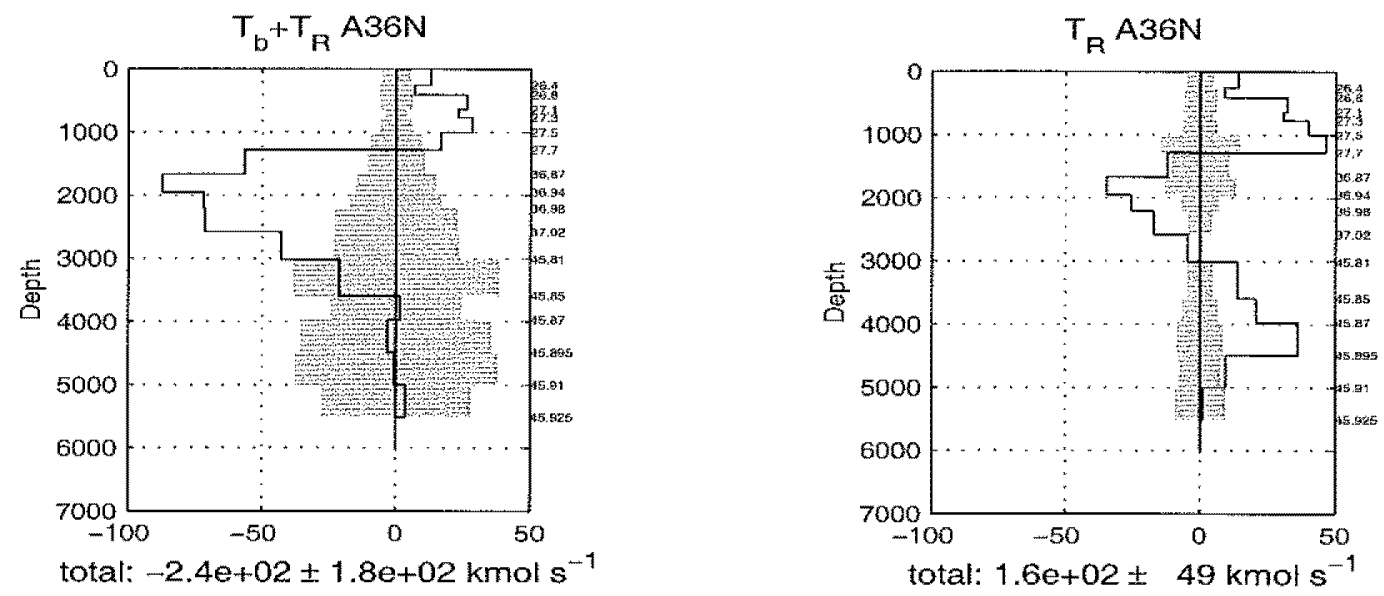

Figure 2-6: Silica variability at $36^{\circ} \mathrm{N}$. The shaded area corresponds to the variability in each layer. The line gives the average transports through each layer in $\mathrm{kmol} \mathrm{Sis}^{-1}$ (see text). The left plot gives the total transport $\left(T_{R}+T_{b}\right)$ while the right plot gives the thermal wind contribution $\left(T_{R}\right)$.

\subsection{Measurement noise}

I now turn on to the measurement noise, $n^{\text {measurement }}$. The measurement noise has two types of components: the first one comes from the temperature and salinity measurements while the second one is due to the approximation in the transports calculations. I first explore the temperature and salinity noise, which is mainly due to internal waves; then I discuss two main approximations that are used: geostrophy and those in the bottom triangles. The error of the average Ekman transport is discussed in the end.

\subsubsection{Internal waves}

Measurement noise in the data from a hydrographic section has at least three different sources: instrumental, navigation and internal waves. Instrumental errors are probably the smallest. CTDs have reached an absolute accuracy of approximately $10^{-3 \circ} \mathrm{C}$ for temperature and better than $4 \times 10^{-3}$ for salinity when carefully calibrated (Saunders, 1986). (The temperature requirements of the WOCE standards is of $0.005^{\circ} \mathrm{C}$ accuracy and $0.002^{\circ} \mathrm{C}$ precision (Joyce and Corry, 1994).) The corresponding noise in the density is $3 \times 10^{-4} \mathrm{~kg} / \mathrm{m}^{3}$ from temperature noise $\left(10^{-3 \circ} \mathrm{C}\right)$ and $3 \times 10^{-3} \mathrm{~kg} / \mathrm{m}^{3}$ from salinity noise $\left(4 \times 10^{-3}\right)$. As 
we will sce below, these deviations are smaller than the noise caused by internal waves. Improperly calibrated CTD's may bias whole data sets by $\pm 0.01^{\circ} \mathrm{C}$ and \pm 0.01 psu (See Marotzke and Willebrand, 1996, p.64 for a review) and cause density shifts over a whole section, with no effect on geostrophic transport calculations. Navigation is also a minor source of error, as it is now very accurate. Moreover, except near high currents (that induce a drift of the rosette during the station) or stcep topography (in the bottom triangle), the errors in station positions to cancel out in transport computations.

The main noise in temperature and salinity measurements is due to internal waves. The transport due to the geostrophic velocity referenced to the bottom between two locations along the section, $S_{w}$ and $S_{e}$, may be written

$$
T=\int_{S_{w}}^{S_{e}} d x \int_{-h}^{0} d z v(x, z)=-\int_{S_{w}}^{S_{e}} d x \int_{-h}^{0} d z \int_{-h}^{z} d \zeta \frac{g}{\rho_{0} f} \frac{\partial \rho}{\partial x}(\zeta, x)
$$

where $h$ is the bottom depth, $\rho$ the density, $g$ the gravity and $f$ the Coriolis parameter. If if the bottom is flat ( $h$ independent of $x$ ), $T$ only depends upon the density measurements at the two end points:

$$
T=\frac{g}{\rho_{0} f} \int_{-h}^{0} d z \int_{-h}^{z} d \zeta\left[\rho\left(\zeta, S_{w}\right)-\rho\left(\zeta, S_{e}\right)\right]
$$

To estimate the noise in $T$, denote the observed transport as $T^{\text {obs }}$ and the true transport as $T^{\text {true }}$. Write

$$
T^{\mathrm{obs}}=T^{\mathrm{true}}+n_{T}
$$

where $n_{T}$ is the observational noise. From (2.11), $n_{T}$ may be written in terms of the noise in the density, $n_{\rho}$ :

$$
n_{T}=\frac{g}{\rho_{0} f} \int_{-h}^{0} d z \int_{-h}^{z} d \zeta\left[n_{\rho}\left(\zeta, S_{w}\right)-n_{\rho}\left(\zeta, S_{e}\right)\right]
$$

so that the variance of $n_{T}$ is

$$
\left\langle n_{T}^{2}\right\rangle=\left(\frac{g}{\rho_{0} f}\right)^{2} \iint_{-h}^{0} d z d z^{\prime} \int_{-h}^{z} \int_{-h}^{z^{\prime}} d \zeta d \zeta^{\prime}\left\langle\left[n_{\rho}\left(\zeta, S_{e}\right)-n_{\rho}\left(\zeta, S_{w}\right)\right]\left[n_{\rho}\left(\zeta^{\prime}, S_{e}\right)-n_{\rho}\left(\zeta^{\prime}, S_{w}\right)\right]\right\rangle,
$$

where the bracket $\langle$ is the expectation. 
Internal waves are one of the best explored fields of physical oceanography and in principle one can compute the full covariance of $n_{\rho}$ using the three dimensional spectra that have been estimated from observations. For my "order of magnitude" error study I used the Garrett-Munk spectrum (Munk, 1981). As observations show no evidence of horizontal coherence over distances larger than $10 \mathrm{~km}$ (Garrett and Munk, 1972), one can assume that $n_{\rho}\left(S_{w}\right)$ and $n_{\rho}\left(S_{e}\right)$ are uncorrelated $\left(S_{w}\right.$ and $S_{e}$ can be taken either between two stations or at the two ends of a section). We then have

$$
\left\langle n_{T}^{2}\right\rangle=\left(\frac{g}{\rho_{0} f}\right)^{2} \int_{-h}^{0} d z \int_{-h}^{0} d z^{\prime} \int_{-h}^{z} d \zeta \int_{-h}^{z^{\prime}} d \zeta^{\prime} \times 2 \times\left\langle n_{\rho}(\zeta) \cdot n_{\rho}\left(\zeta^{\prime}\right)\right\rangle
$$

To compute the integrals, the water column is divided into segments $\Delta z_{i}$ of $500 \mathrm{~m}$ or more so that the internal wave noise is approximately uncorrelated between each segment. Eq. (2.12) is evaluated as

$$
\begin{aligned}
n_{T} & =\frac{g}{\rho_{0} f} \sum_{k=1}^{M} \Delta z_{k} \sum_{i=1}^{k} \Delta z_{i} \times\left[n_{\rho}\left(z_{i}, S e\right)-n_{\rho}\left(z_{i}, S w\right)\right] \\
& =\frac{g}{\rho_{0} f} \times \mathbf{a}^{\mathrm{T}} \mathbf{K}\left[\mathbf{n}_{\rho}(S e)-\mathbf{n}_{\rho}(S w)\right]
\end{aligned}
$$

where the column vector $\mathbf{n}_{\rho}$ contains $n_{\rho}\left(z_{i}\right)$ and the vector-matrix product $\mathbf{a}^{\mathrm{T}} \mathbf{K}$ corresponds to the two sums. $\Delta z_{k}$ is the length of segment $k$. Then, (2.13) may be evaluated

$$
\left\langle n_{T}^{2}\right\rangle=2 \times\left(\frac{g}{\rho_{0} f}\right)^{2} \mathbf{a}^{\mathrm{T}} \mathbf{K}\left\langle\mathbf{n}_{\rho} \mathbf{n}_{\rho}^{\mathrm{T}}\right\rangle \mathbf{K}^{\mathrm{T}} \mathbf{a}
$$

where the covariance matrix $\left\langle\mathbf{n}_{\rho} \mathbf{n}_{\rho}^{\mathrm{T}}\right\rangle$ is diagonal. The elements of $\left\langle\mathbf{n}_{\rho} \mathbf{n}_{\rho}^{\mathrm{T}}\right\rangle$, namely $\left\langle n_{\rho}^{2}\right\rangle\left\langle z_{i}\right\rangle$, are chosen for each depth following Marotzke and Willebrand (1996b) with slight modifications. First, the variance of the isopycnal displacements is estimated using the Munk (1981) formula,

$$
\left\langle\xi^{2}\right\rangle=53 \frac{N_{0}}{N(z)}\left(\text { in } \mathrm{m}^{2}\right)
$$

where $N_{0}=5.2 \times 10^{-3} \mathrm{~s}^{-1}$ and $N$ is the local Brunt-Väisälä frequency. The conversion into density variance gives

$$
\left\langle n_{\rho}^{2}\right\rangle=53\left(\frac{\rho_{0}}{g}\right)^{2} \times N_{0} N(z)^{3}
$$


$N$ varies from $0.8 \times 10^{-3} \mathrm{~s}^{-1}$ in the deep layers to $5 \times 10^{-3} \mathrm{~s}^{-1}$ in the thermocline at midlatitudes (e.g., Gill, 1982, p.52). The corresponding noise $n_{\rho}$ goes from $0.0013 \mathrm{~kg} / \mathrm{m}^{3} \mathrm{rms}$ to $0.02 \mathrm{~kg} / \mathrm{m}^{3} \mathrm{rms}$. Because it is tiny in the deep ocean, $n_{\rho}$ was given a lower bound equivalent to the CTD noise $\left(0.003 \mathrm{~kg} \mathrm{~m}^{-3} \mathrm{rms}\right)$. At a latitude of $30^{\circ}$ and for $h=4000 \mathrm{~m}$, the rms noise in the transport (Eq.2.14) was integrated using $500 \mathrm{~m}$ segments. The resulting noise in the total transport is $3 \mathrm{~Sv} r m s$. This result is found insensitive to a change in the segment of integration, and a depth of $h=5000 \mathrm{~m}$ produces the same order of magnitude. The noise at different latitudes is obtained by scaling the $\pm 3 \mathrm{~Sv}$ rms with the appropriate ratio of Coriolis parameter.

Fieux et al. (1996a) observed large internal fluctuations in the transports between repeat stations taken a few hours apart in the Bali region. The authors suspected that these fluctuations were due to internal wave activity. They found variations of order $9 \mathrm{~Sv}$, comparable to the noise of $\pm 10 \mathrm{~Sv}$ predicted by Eq. (2.14) at this latitude $\left(10^{\circ}\right)$.

\subsubsection{Geostrophy}

In the ocean interior, and except for the Ekman layer, geostrophy is recognized as a good approximation. Even in the western boundary current, equation scaling shows that the along-stream velocity is in geostrophic equilibrium (see Pedlosky, 1987, Chapter 8). The very first test of geostrophy is probably the one of Wüst (1924) who compared currentmeter data with the geostrophic velocities in the Florida Current and found a qualitative agreement (see also Wunsch, 1996, p.47). Recently, Johns et al.(1989) directly tested geostrophy in the Gulf-Stream at $36^{\circ} \mathrm{N}$ using Pegasus profiler currents. They obtained an agreement within $3 \mathrm{~Sv}$ between the absolute and geostrophic transports above $2000 \mathrm{dbars}$, only a $3 \%$ error in the total transport of $93 \mathrm{~Sv}$. Using a satellite image of the Gulf-Stream position at the time of the experiment, the authors estimated the correction from the cyclostrophic term to be about $2 \mathrm{~Sv}$, which further reduced the discrepancy to $1 \mathrm{~Sv}$. This result is most encouraging, although the measurement errors are probably much larger.

In addition to the cyclostrophic term, ageostrophic motions include the energetic inertial oscillations. The extent to which inertial motion is able to carry energy or other tracers when integrated over a section is not trivial in presence of the topography and the 
planetary vorticity gradient. Ripa (1997) gives the analytical description of inertial oscillations in the presence of the planetary vorticity gradient. For a surface inertial velocity of order $V=10 \mathrm{~cm} \mathrm{~s}^{-1}$, the inertial circles have a radius $R=V / f=1000 \mathrm{~m}$ at mid-latitudes and a westward "Stokes" drift appears (Ripa, 1997)

$$
\langle\dot{x}\rangle=-\frac{1}{2} \beta R^{2} \sim .5 \times 10^{-11} \times 10^{6}=O\left(5 \times 10^{-4} \mathrm{~cm} \mathrm{~s}^{-1}\right)
$$

where $\beta$ is the planetary vorticity gradient. The magnitude is much smaller than the geostrophic horizontal advection and purely zonal with no implications for the meridional tracer transports.

A second effect of the planetary vorticity gradient is to shift the inertial frequency slightly above $f$ (e.g., Munk, 1980). The pressure field is affected ageostrophically, corrupting the hydrographic measurements. Such a peak above the inertial frequency has been observed in some of the internal wave potential energy spectra (Fu, 1981). However, the author noted that the peak may be spurious and a result of inertial motion of the moorings themselves. I do not know how much this ageostrophic effect aliases transport calculations, and it is assumed that the implied error does not exceed the internal wave error of Section 2.3.1.

In conclusion, the effect of inertial motion is much smaller than other uncertainties in the budget. Still, a $\pm 2 \mathrm{~Sv}$ rms uncertainty may be attributed to the cyclostrophic term across the Gulf-Stream at $36^{\circ} \mathrm{N}$.

\subsubsection{Bottom triangle}

In the presence of sloping topography one has to decide how to compute geostrophic velocities and property transports between two stations near the bottom. The situation for an actual hydrographic line is sketched on Figure 2-8a. For computing geostrophic transports, one usually extrapolates the temperature, salinity and other properties beneath the deepest common level of the two stations (noted hereafter DCL). For instance, at the two first stations of Fig. $2-8 a$, the temperature was measured on the black dots in the triangle below the DCL. It must be extrapolated along the bottom slope or, equivalently, 
a)

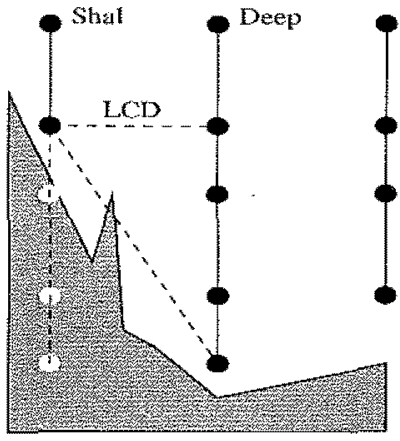

b)

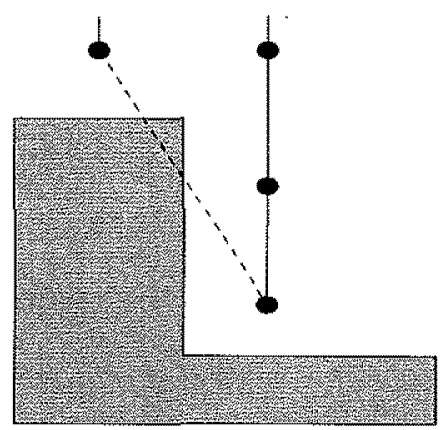

c)

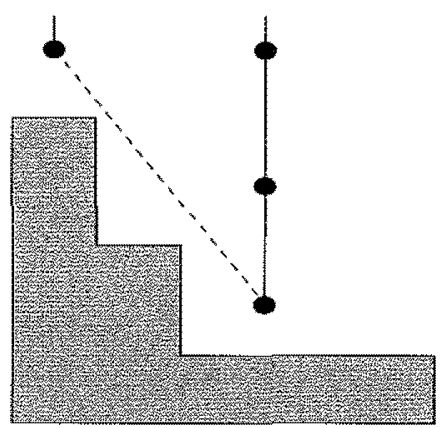

Figure 2-8: Bottom triangle problem. The topography is shaded. the first line corresponds to the shallow station, the second line to the deep station. The black dots are temperature measurements, a) in the real ocean; b) in the model; c) in the subsampled model. DCL stands for the deepest common level.

on the imaginary points in white that are below the DCL at the shallow station.

The temperatures at the white points are denoted $T_{\text {shal }}$ while the temperatures at the deep station and below the DCL are noted $T_{\text {deep. }}$. The salinities $S_{\text {deep }}$ and $S_{\text {shal }}$ that are extrapolated with the same method as temperatures and the geostrophic velocities are computed using the extrapolated $T_{\text {shal }}$ and the data $T_{\text {deep }}$ and $S_{\text {shal }}, S_{\text {deep }}$. The transport is computed by integration of the velocity in the triangle.

Uncertainties arise from both the extrapolation of $T_{\text {shal }}$ and the estimation of the area in the bottom triangle. Here, I estimate the uncertainties related to the extrapolation only by comparing six methods:

- Method 1: null velocity below the DCL. The transport in the triangle is ignored.

- Method 2: no shear. $T_{\text {shai }}$ is set equal to $T_{\text {deep }}$ at each depth so that the geostrophic velocity is constant below the DCL.

- Method 3: constant slope. The slope of the isotherm just above DCL is calculated. $T_{s h a l}$ is computed using the same slope (vertical variations to horizontal variations). The magnitude of the slope is limited to an upper bound of 1 (regardless of the vertical to horizontal distance ratio) to exclude excessively large shears.

- Method 4: plane fit (suggested by Wunsch, 1996). A linear function is determined 
by regression between the temperature data, the depth and distance on the triangle (black dots):

$$
T(x, z)=a x+b z+c
$$

where $x$ is the distance to the deep station. The relation gives $T_{\text {shal }}$.

- Method 5: polynomial fit. Same as the plane fit, but a higher order polynomial is used in the $z$ direction:

$$
T(x, z)=a x+b z+c z^{2}+d .
$$

The polynomial is useful in regions of strong $T$ curvature (e.g., near the thermocline).

- Method 6: horizontal extrapolation. Temperature is extrapolated linearly from the next deeper station.

Other extrapolation methods such as mapping have been used in the literature (e.g., Roemmich and Wunsch, 1985), all bearing their own advantages and drawbacks. To determine the possible error caused by the extrapolation, the relative geostrophic transports were computed using each one of the six methods listed above and across three sections in the Atlantic: $24^{\circ} \mathrm{N}$ (Morocco to east of the Bahamas Bank); Florida Strait (hereafter Flst); $36^{\circ} \mathrm{N}$ and $48^{\circ} \mathrm{N}$. The geostrophic velocities were referenced to deep isopycnals: $\sigma_{4000}=45.81$ at $24^{\circ} \mathrm{N}$ and $36^{\circ} \mathrm{N}$ and $\sigma_{2000}=36.87$ at $48^{\circ} \mathrm{N}$ (from Macdonald, 1995) or to the bottom if shallower than the target isopycnal. For those "shallow stations", the transport calculation was done with the velocity referenced 1) at the depth of the deepest station and 2) at the DCL of the two stations. The total relative transports are displayed on Figure 2-9 for each method and in the two cases.

In the first case (Fig. 2-9, upper panel) the relative transports vary from 6 to $31 \mathrm{~Sv}$ at $24^{\circ} \mathrm{N}$ depending upon the method. While it is relatively stable in the Florida Strait $( \pm 2 \mathrm{~Sv})$ it exhibits large variations at $36^{\circ} \mathrm{N}(+45 \mathrm{~Sv})$ and at $48^{\circ} \mathrm{N}(+5 \mathrm{~Sv})$. Figure 2-10, lower panel, shows the transports cumulated from the eastern boundary at $36^{\circ} \mathrm{N}$ for each method. The discrepancies appear almost exclusively in the regions where the velocity is referenced to the bottom. Because the velocity there is referenced to the deepest station depth, any 


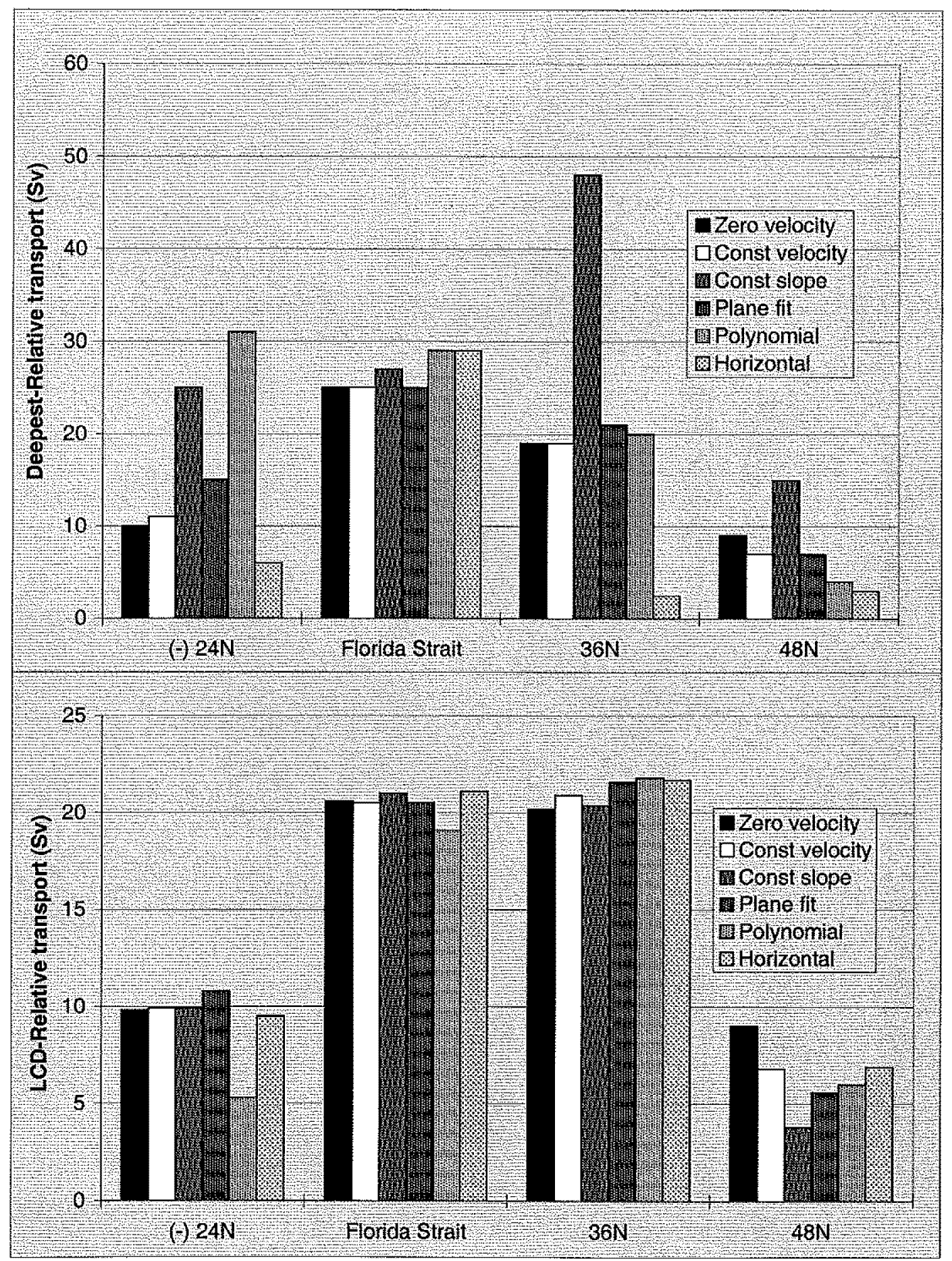

Figure 2-9: Net relative transport across each section and for different bottom wedge methods. The calculation is much more sensitive to the method if the reference in shallow waters is taken at the deepest stations (upper panel) than at the last common depth (lower panel). 
error in the bottom triangle is propagated through the whole water column. An extreme example occurs along the eastern slope (Fig.2-10): Between 1000 and 2000 dbars, a strong density gradient due to the Mediterranean outflow coincides with a steep bottom slope. As a result, the shear between the DCL and the bottom depth varies by $1 \mathrm{~ms}^{-1}$ depending upon the method, introducing a $30 \mathrm{~Sv}$ anomaly with the "constant slope" method. While this extreme anomaly in the velocity field is readily identified and corrected, smaller errors are not obvious while their effect may be important.

A straightforward way to minimize the error induced by the bottom wedge extrapolation is to reference the "shallow pairs" to the DCL instead of the deepest station. Then, the uncertainty in the bottom triangles has no effect on the water column above DCL. The transports in this case are displayed on Fig.2-9, lower panel. (Even if the reference isopycnal was between the DCL and the deepest station, the reference was taken at the DCL.) Within $1 \mathrm{~Sv}$, and except for the polynomial fit at $24^{\circ} \mathrm{N}$, the relative transports are independent of the method at $24^{\circ} \mathrm{N}$, in the Florida Strait, and at $36^{\circ} \mathrm{N}$. At $48^{\circ} \mathrm{N}$, the scatter is more important ( 4 to $9 \mathrm{~Sv}$ ) because of the presence the deep western boundary (DWBC) across several bottom triangles.

The choice of an extrapolation method is somewhat arbitrary. In principle, one could use the GCM output, where the answer is known, to select the best method. Unfortunately, the GCM configuration is inadequate: Because of the non-slip condition at horizontal boundaries, the actual velocity in the triangle-which is centered on a velocity point-is null (Fig.2-8b). Alternatively, the GCM may be sub-sampled at every other grid points (Fig. 2-8c), but with the present model resolution, the resulting station spacing would be much larger than it is in actual hydrographic data.

Experiments on many hydrographic sections showed that methods $2-5$ (constant velocity, constant slope, plane fit and polynomial fit) propagate the temperature and velocity structure downwards in the water column. If the depth difference between the two stations is large, unrealistic temperature gradients and velocity shears can be introduced at depth in the bottom triangle (e.g., if the shallow station is in the thermocline and the deep station is at 3000 dbars). Ignoring the transports in the triangles may be safer but not realistic, in particular when it implies a large discontinuous shear at the DCL. The "con- 

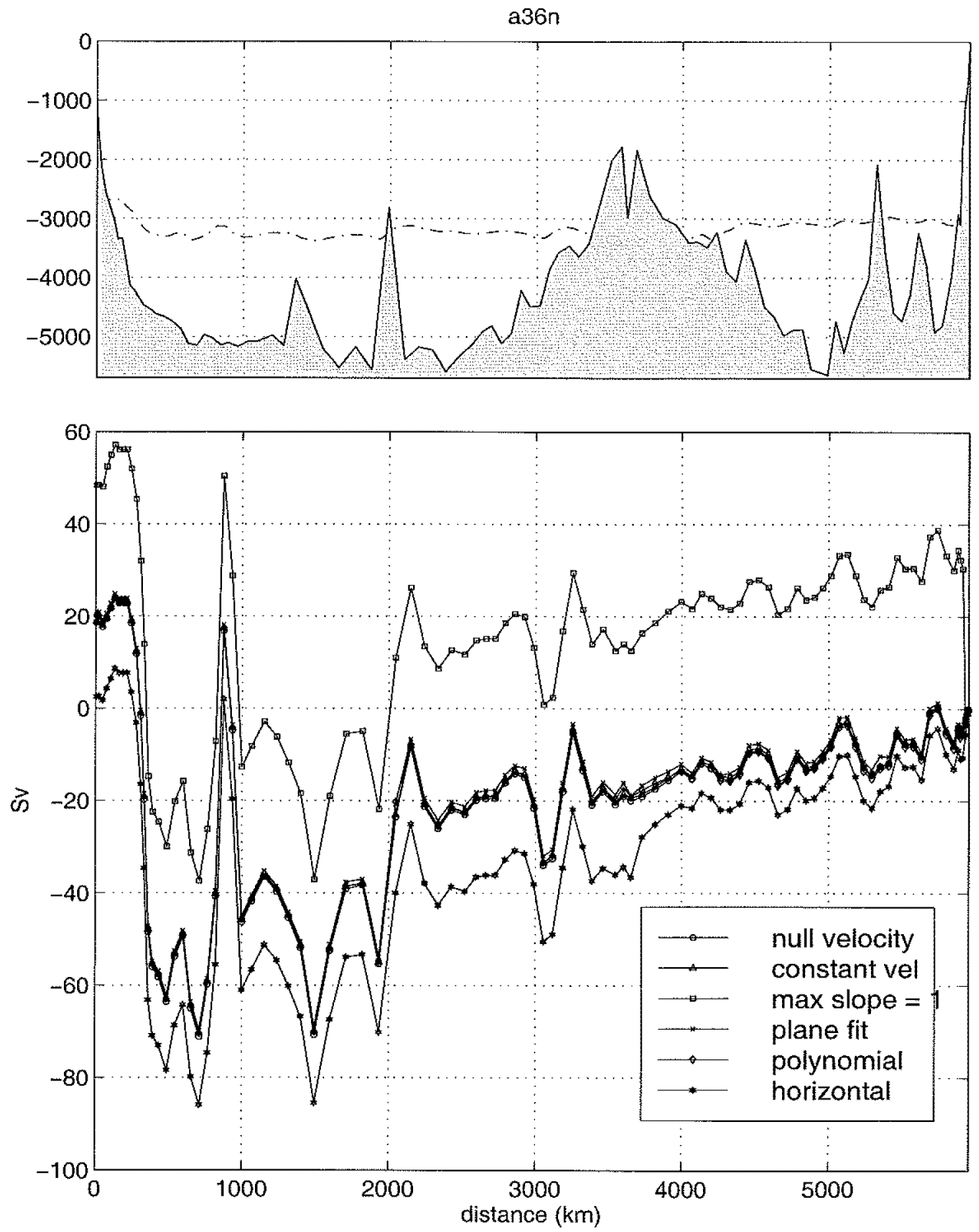

Figure 2-10: Relative transport at $36^{\circ} \mathrm{N}$ integrated from east. The reference surface is indicated by the dash-dot line on the upper panel. The transports in the bottom triangles are computed with different methods. The "shallow" pairs are referenced to the deepest station, causing the large discrepancies. 
stant velocity" method is relatively safe, but it implies an abrupt flattening of isopycnals, which is not necessarily realistic either. On the other hand, the horizontal extrapolation has the advantage of reproducing the velocity shear from the next deeper station pair, which is by definition realistic. This method may be unstable in the rare case of two closely spaced stations followed by a distant station along a rising bottom slope. Then, the noise in the close stations is amplified through the extrapolation to the distant station. For those particular cases, the extrapolation may be done manually, using for instance the "constant velocity" method. For stations where no horizontal extrapolation is possible (i.e., the station is isolated in a trench), the polynomial fit turns out to produce the most realistic-looking temperatures as long as the extrapolation depth interval is small, because the technique permits curvature in the extrapolated temperatures.

In conclusion, my estimation of the error due to the bottom wedge problem on a given transoceanic section is of order $1 \mathrm{~Sv}$ as long as the reference level of shallow stations is taken at the deepest common level of the two stations. In the presence of a boundary current along sloping topography, this error is probably, 2 to $4 \mathrm{~Sv}$. Because this estimate derives from a comparison between various extrapolation methods and a null bottom triangle transport, it is likely to be an overestimate of the actual error. Further tests may be carried out to estimate the bottom triangle error (e.g., subsampling a high resolution section or numerical model; comparing calculations with LADCP measurements).

\subsubsection{Mean Ekman transport uncertainty}

As pointed in Section 2.2.2 it is appropriate to use the mean Ekman transport rather than the instantaneous value in property budgets. In Section 2.2 .2 , it was shown that the classical Ekman formula (2.5, page 28) was adequate for estimating the net Ekman transport in the GCM. However, due to the large variability in the Ekman layer, Eq.(2.5) has no definite support from observation. But this relation between the wind stress and its implied mass transport has never been proven wrong. The few existing measurements are encouraging (e.g., Chereskin, 1995; Price et al., 1988; Schludlich and Price, 1998; Weller and Pueddemann, 1996). A convincing test of the net meridional Ekman transport comes from Chereskin and Roemmich (1991) who compared, along a hydrographic section $\left(11^{\circ} \mathrm{N}\right.$ 
in the Atlantic), the total Ekman transport (Eq.2.5) with a direct calculation of the surface ageostrophic component based on hydrographic and ADCP measurements. The different estimates agreed within their estimated error bars, $12 \pm 5 \mathrm{~Sv}$ from the ADCP against $13.5 \pm 0.3 \mathrm{~Sv}$ from (2.5). Wijffels et al. (1994) arrived to a similar conclusion at $10^{\circ} \mathrm{N}$ in the Pacific Ocean.

The accuracy of the wind stress itself is uncertain. It depends on 1) the accuracy of the estimated wind itself and, 2) the validity of the momentum transfer parameterization at the sea surface. Mestas-Nunez et al. (1994) compared the monthly wind stresses from the climatology of Trenberth et al. (1990) with the Seasat-A Satellite Scaterrometer (SASS) stresses. They found that the Trenberth stresses were overestimated by $46 \%$ in most oceanic regions, except at the Equator and at high southern latitudes where the errors became larger. Most of the difference was explained because Trenberth et al. (1990) used the 1000-mbars winds to compute the stress instead of the $10-\mathrm{m}$ winds. Huang and Shukla (1996) arrived at a similar conclusion in comparing ECMWF to ship wind observations in the tropical Atlantic. Note that the ECMWF wind stresses that were used in Section 2.2.2 are obtained from a Monin-Obukhov-type boundary layer parametrization (ECMWF forecast model physical parametrization, 1991) which is probably more accurate than the drag-coefficient-based analysis of Trenberth et al. (1990) .

In the present hydrographic inverse model, the mean Ekman transports were computed from the NCEP re-analysis (Kalnay et al., 1996). It is difficult to provide a reliable number for the a priori uncertainty on the average Ekman transport because of the uncertainty in both the wind stress and the calculation itself (Eq.2.5). Given the discrepancies cited above $(46 \%)$, an uncertainty of $50 \%$ was attributed to the mean Ekman transport. For sections of low Ekman transport, the uncertainty was given a lower bound of $1 \mathrm{~Sv}$.

Because of possible correlations between velocity and temperature in the Ekman layer, the error in the energy flux calculation in the Ekman layer can be much larger than that in the mass flux itself. Variability in the net energy flux through a trans-oceanic section is dominated by the vertical temperature gradients while the gyre component is a minor contribution (Jayne, 1999). A sensible test of the average energy transport would be to compare $\overline{v T}$ and $\bar{v} \bar{T}$ (where $T$ is temperature and the overbar is the horizontal average) 
from ADCP data as in the study by Chereskin and Roemmich (1991). But this is beyond the present work, and I assume that the uncertainty in $\bar{v} \bar{T}$ includes any possible eddy transports $\overline{v^{\prime} T}$. Nutrient fluxes are much less affected by horizontal fluctuations in the Ekman layer because nutrients tend to be depleted near the surface 


\subsection{Reference level velocity variability}

In principle, the a priori reference velocity covariance, $\left\langle\mathbf{b b}^{T}\right\rangle=\left\langle b(x) b\left(x^{\prime}\right)\right\rangle$, could be fully determined from the GCM. However, the calculation is complicated because $\left\langle\mathbf{b b}^{T}\right\rangle$ is likely to be non-isotropic and geographically dependent. Moreover, there is no guarantee that the GCM produces the true oceanic covariance structure because it does not entirely resolve oceanic eddies. Consequently, I make the assumption of no correlation between elements, that is, a diagonal covariance matrix. To provide at least a lower bound for the diagonal elements of $\left\langle\mathbf{b} \mathbf{b}^{T}\right\rangle$, the GCM output is examined.

At the three latitudes $24^{\circ} \mathrm{N}, 36^{\circ} \mathrm{N}$ and $48^{\circ} \mathrm{N}$, the absolute geostrophic velocity was sampled at the reference isopycnal every third day in the GCM. (As above, I took the reference isopycnals $\sigma_{4}=45.81$ at $24^{\circ} \mathrm{N}$ and $36^{\circ} \mathrm{N}$ and $\sigma_{2}=36.87$ at $48^{\circ} \mathrm{N}$ from Macdonald, 1995.)

Figure 2-11, upper panel shows the average reference isopycnal position at $36^{\circ} \mathrm{N}\left(\sigma_{4}=\right.$ 45.81 , thick line) over 8 years. The envelope defining one standard deviation of the isopycnal displacements is indicated by the thin line. Deviations are lower than $\pm 100 \mathrm{~m}$, suggesting that the variations in the reference velocity are thus mainly due to variations in the velocity field rather than related to reference isopycnal displacement over time.

The time-mean reference velocities (Figure 2-11, lower panel, thick line) are generally smaller than $0.2 \mathrm{~cm} \mathrm{~s}^{-1}$ in the oceanic interior while the variability, indicated by the shaded area, is approximately $\pm 0.5 \mathrm{~cm} \mathrm{~s}^{-1} \mathrm{rms}$. The mean reference velocities increase to $20 \mathrm{~cm} \mathrm{~s}^{-1}$ near the western boundary and to $5 \mathrm{~cm} \mathrm{~s}^{-1}$ in shallow waters to the east. The situation is similar at $24^{\circ} \mathrm{N}$ (not shown) while at $48^{\circ} \mathrm{N}$ the amplitude of both the mean and the variability are twice as large. At this latitude, Macdonald (1995) chose a relatively shallow isopycnal of reference $\left(\sigma_{2}=36.87,1200-2000 \mathrm{~m}\right)$. Nevertheless, the reference velocities on a deeper isopycnal $\left(\sigma_{4}=45.81,3000-3600 \mathrm{~m}\right)$ have the same amplitude and variability. Mean velocities of up to $6 \mathrm{cms}^{-1}$ are found in the deep western boundary current at $48^{\circ} \mathrm{N}$ near the continental slope.

It is not clear whether the mean reference velocity size, its variability, or its rms is the right a priori covariance for $b$. On one hand, the variability contains the fast responses to Ekman transport fluctuations. Those are filtered out in the mass balance by specifying the 

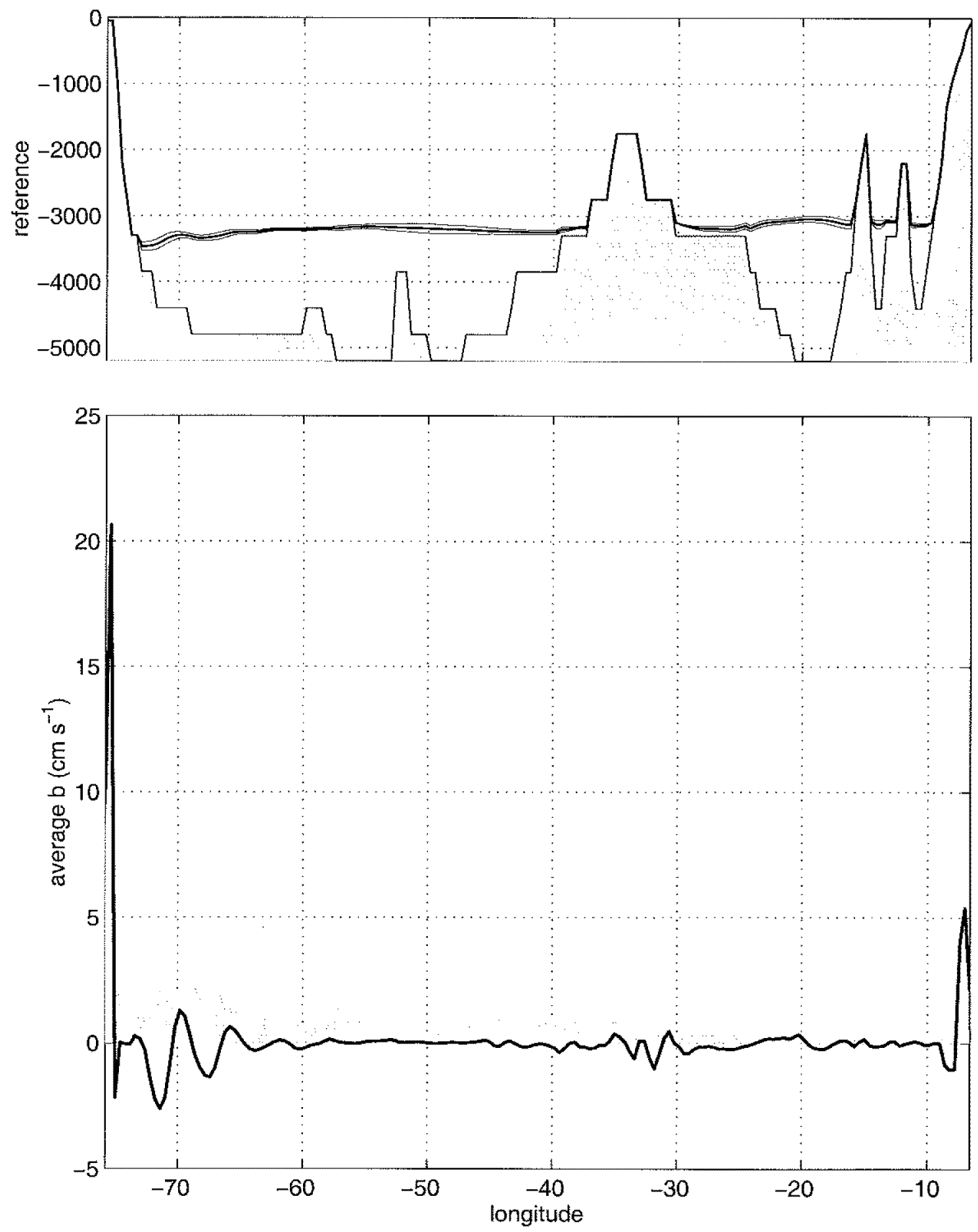

Figure 2-11: Upper panel: reference isopycnal $\left(\sigma_{4}=45.81\right)$ for section $36^{\circ} \mathrm{N}$. The isopycnal oscillates by less than $100 \mathrm{~m}$ around its mean position (thick line). One standard deviation is indicated by the thin lines. Lower panel: mean reference velocities (thick line) and variance (1std. dev, shaded area). 
time-mean Ekman transport (see the introduction, Section 2.1). On the other hand, the model variability in the reference velocities also contains the eddy part, both baroclinic and barotropic: We have seen that the relative transport $T_{R}(t)$ is stable when integrated on large scales. However, $T_{R}$ contains baroclinic eddies that have a signature in the reference velocities (unless all eddy velocities somehow vanish at the reference depth). As a result, the estimated mean transport $\overline{T_{b}}$ may contain some eddy signals to be consistent with the instantaneous realization $T_{R}(t)$.

In general though, the reference velocity and its variability have similar magnitude. Because the GCM reproduces about half the actual oceanic variability (see Section 2.2), an a priori value for the reference velocity variance in the interior of $1 \mathrm{~cm}^{2} \mathrm{~s}^{-2}$, as used by Macdonald (1995) was adopted. In shallow waters and near boundary currents, this a priori value is increased according to current meter statistics when available. Except in boundary currents where an accurate reference surface could be determined from current meter measurements, the relative velocity variations within a range of about 500 dbars was taken as the a priori value to reflect the uncertainty in the reference surface choice, with a minimum of $1 \mathrm{~cm}^{2} \mathrm{~s}^{-2}$. 


\subsection{Summary}

This evaluation of the a priori uncertainty in mass conservation equations of linear inverse models shows that:

1. The variability in the absolute geostrophic velocity field is dominated by fast, depthindependent responses to Ekman transport fluctuations. Consequently, linear inverse models can consistently combine different sections as long as the mean Ekman transport is used for all sections.

2. The baroclinic shear is relatively stable on large scales (at least in the GCM that was examined) so that the relative baroclinic transport measured at a particular time is representative of the time-mean. Still, the error implied by baroclinic variations is not negligible, with a total of $\pm 5 \mathrm{~Sv}$ at mid-latitudes for the top-to-bottom transport and $\pm 0.1 \pm 2 \mathrm{~Sv}$ in the selected individual layers. The finite time required by a ship to cross the ocean does not seem to introduce any significant bias.

3. The measurement noise is dominated by internal wave vertical heave of isopycnals, implying a $\pm 3 \mathrm{~Sv}$ error in the transport estimates at mid-latitudes. The geostrophic assumption and the bottom triangle issues induce smaller error except in very specific situations such as a boundary current with large curvature or a deep boundary current against a slope.

Table 2.2 summarizes the a priori uncertainties on the top-to-bottom flow and on the flow in isopycnal layers above and below $2000 \mathrm{dbar}$. (The layers were defined in Section 2.2.3.) The uncertainty in the top-to-bottom flow is dominated by the baroclinic variability of $\pm 5 \mathrm{~Sv}$. Assuming that the different uncertainties are independent, the total rms uncertainty is $6 \mathrm{~Sv}$. In a conservation equation involving two sections, the noise level, assuming uncorrelated errors between the sections, would be $8.5 \mathrm{~Sv}$ rms, suggesting a 4 -fold increase of the $2 \mathrm{~Sv}$ rms uncertainty adopted by Rintoul (1988) and Macdonald (1995).

Using such a large error for the net flux through a section is somewhat counter-intuitive as it is known, independently from inverse models and hydrographic data, that mass is conserved within the uncertainty on the freshwater flux divergence, $\pm 1 \mathrm{~Sv}$ or less. But 
because the model is linear, with no adjustment to density, the model error due to the variability in the density field must be included to obtain reliable estimates and uncertainties for the time-mean property fluxes. Figure 2-12 illustrates the effect of imposing stringent mass conservation between two sections in the presence of baroclinic noise in a two-dimensional case. The true average is an overturning of $5 \mathrm{~Sv}$ (upper panel). Because of baroclinic variability in the upper layer, a shear of only $5 \mathrm{~Sv}$ is observed in the first section (red dashed line). The second section is supposed to be perfectly measured. In the lower layer, the baroclinic variability is much weaker. If one enforces top-to-bottom mass conservation, the derived circulation (middle panel of Figure 2-12) has half the overturning strength and spurious vertical transfers are implied by mass conservation between the two sections. The weak overturning is probably the true circulation at the time of the hydrographic section; however, it is a misrepresentation of the time-mean, leading to erroneous vertical transfers. But according to the present GCM simulation, the baroclinic shear is more stable in the deep layers. By ensuring a better conservation in the deep layer at the expense of the top-to-bottom conservation, one gets a circulation which is representative of the time average but it contains mass residuals in the upper layer (lower panel of Fig. 2-12). This solution is correct as long as the mass residuals are indistinguishable from zero within uncertainties. A numerical illustration in a two-dimensional ocean is given in Appendix B.

Along with mass, the model error for the silica flux was estimated because silica is a useful tracer to constrain the circulation (e.g., Rintoul and Wunsch, 1991). The uncertainty due to the baroclinic variability in the GCM output was estimated at $\pm 50 \mathrm{kmol} \mathrm{Si} \mathrm{s}^{-1}$ top-to-bottom and at $\pm 10 \mathrm{kmol} \mathrm{Sis}^{-1}$ in individual layers.

The a priori reference velocity magnitude was evaluated from the GCM as lying between \pm 0.5 and $\pm 1 \mathrm{~cm} \mathrm{~s}^{-1}$. In shallow waters and near boundary currents, the values are much larger. Practically, an a priori value of $\pm 1 \mathrm{cms}^{-1}$ will be used as a lower bound and modified according to 1) current-meter measurements where available and 2) the local shear to account for the uncertainty due to the reference surface choice. In principle it is possible to use the spatial structure of the reference level velocity as prior information. For example, one can minimize the solution smoothness instead of its variance, which is 


\section{Conservation in the deep layers versus top-to-bottom}

\section{1) True average}
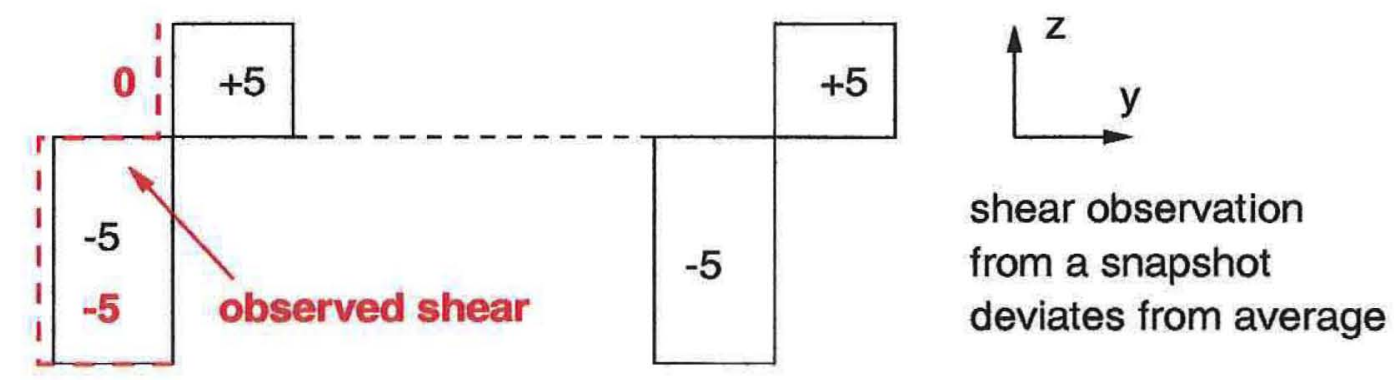

2) Enforce top-to-bottom conservation

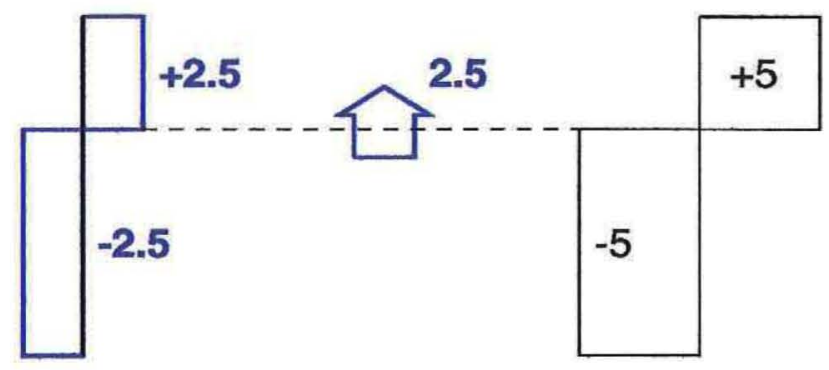

true instantaneous

circulation but

variability in upper

layers induces spurious

vertical transfers

\section{3) Enforce deep layer conservation}

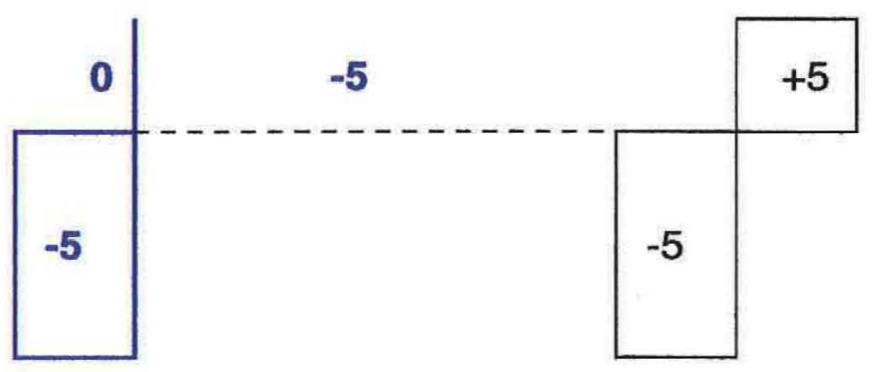

deep layer circulation is stable; residual due to noise in upper layers

Figure 2-12: Enhancing conservation in the deep layers at the expense of the top-to-bottom mass budget: two-dimensional example. Panel 1) shows the true average circulation, an overturning of $5 \mathrm{~Sv}$ at both sections. The red dotted line corresponds to the observation of the section to the left. Panel 2) and 3) are two estimates of the circulation that conserve mass (1) top-to-bottom and (2) in the deep layer. 


\begin{tabular}{|l|c|c|c|}
\hline & Top-to bottom & Layer above $2000 \mathrm{~m}$ & Layer below 2000 \\
\hline Variability & 5 & $1-2$ & 0.5 \\
\hline Internal waves & 3 & - & 0.2 \\
\hline Geostrophy (Gulf-Stream) & 2 & - & - \\
\hline Bottom wedge & 1 & - & - \\
\hline \hline Total error & 6 & $1-2$ & 0.5 \\
\hline
\end{tabular}

Table 2.2: Error budget across the $36^{\circ} \mathrm{N}$ Atlantic section. The a priori uncertainties are provided on the total flux and in layers above and below $2000 \mathrm{db}$ ( $\mathrm{Sv} \mathrm{rms}$ ). The geostrophic error comes from the Gulf-Stream curvature at $36^{\circ} \mathrm{N}$.

equivalent to requiring a solution in which the large scales are more energetic than the small scales by choosing accordingly the covariance $\left\langle\mathbf{b b}^{T}\right\rangle$. However, the spectrum varies substantially with location and a white noise assumption is the most practical prior choice.

The results are dependent upon the skill of the GCM in representing the true ocean. On the one hand, the GCM velocity variability is lower than the true variability by a factor of 2 to 4 (Stammer et al., 1996) although we do not know what are the respective fractions of baroclinic and barotropic variability that are represented. On the other hand, the GCM is known to contain a drift term. The drift was not corrected and will introduce spurious variations in the density field (Marotzke, personal communication, 1998). Nevertheless, the uncertainties are in agreement with an analysis of the few sections that were repeated in the Atlantic within a factor of two, which is sufficiently accurate for an a priori uncertainty estimate.

Another component of the model error is the Eulerian nature of the transports calculation. Eulerian tracer transports are an approximation to the true Lagrangian property transports. This approximation may be a source of noise in tracer transport calculations and budgets. The problem is too vast to be addressed in the present context and I am left with the assumption that the error implied does not greatly exceed the error from the variability.

The uncertainty attributable to a mass conservation equation involving several sections depends upon the correlation between the errors in the flux across each section. Measurement errors are probably uncorrelated from one section to another. In the model, the correlation between the net relative transport through $24^{\circ} \mathrm{N}, 36^{\circ} \mathrm{N}$ and $48^{\circ} \mathrm{N}$ in the 
GCM was calculated over the 8-year time series. The maximum correlation was found between $36^{\circ} \mathrm{N}$ and $48^{\circ} \mathrm{N}$ with a value of 0.24 , which is not significant as the $95 \%$ non-zero correlation is 0.35 . (Each time series of relative transport had a decorrelation scale of 100 days, leaving only 30 independent samples.) A coherence analysis showed no significant correlation at any time scale. Consequently, one can reasonably assume that the uncertainties are uncorrelated from one section to the next and sum the error variances of sections entering a mass conservation equation.

Conversely, variations in different layers of a section are probably correlated. Roemmich and Wunsch (1985) found systematic deviations in comparing data from sections taken years apart. This correlation is likely to depend upon many parameters like depth, presence of a boundary current in the layer, latitude, etc. For simplicity, and due to the lack of information on those correlations, it was decided to leave the a priori uncertainties uncorrelated between layers.

\subsection{Application to the global inversion}

In principle, with a global GCM, one could repeat the error budget analysis in each oceanic region. But to determine the a priori uncertainties that are needed, it was judged sufficient, to adapt the results in the North Atlantic to other oceans and to low latitudes.

Geostrophy has never been proved to fail either from scaling arguments or from observations (Eriksen, 1982), even within a few degrees of the Equator. To extrapolate a general rule based upon the present study, the uncertainty from measurement noise (mainly internal waves) was scaled by the inverse of the Coriolis parameter. The measurement noise goes from $\pm 2 \mathrm{~Sv}$ at $50^{\circ} \mathrm{N}$ to $\pm 20 \mathrm{~Sv}$ at $5^{\circ} \mathrm{N}$ (Table 2.3 , second column). Because of geostrophy, this transport error is independent of the distance between stations.

The model error due to variability does not seem to depend directly upon the Coriolis parameter in any of the three North Atlantic sections in the GCM and the extrapolation at low latitudes were obtained by assuming, as a zero-order guess, that the variability had about the same contribution as measurement errors on the total budget (Table 2.3, third column). The variability in individual layers was scaled with the Coriolis parameter. The 


\begin{tabular}{|c|c|c|c|c|c|}
\hline \multirow{2}{*}{ Latitude } & \multicolumn{2}{|c|}{ Total flux } & \multicolumn{3}{c|}{$\begin{array}{c}\text { Individual layers } \\
\text { variability }\end{array}$} \\
\cline { 2 - 6 } & $\begin{array}{c}\text { meas. } \\
\text { noise }\end{array}$ & (variability) & & \multicolumn{3}{|c|}{0 -1000db } & $1000-2000 \mathrm{db}$ & $2000 \mathrm{db}$-bottom \\
\hline \hline 50 & 2 & 2 & 2 & 1 & .5 \\
\hline 40 & 3 & 4 & 2 & 1 & .5 \\
\hline 30 & 3 & 6 & 2 & 1 & .5 \\
\hline 20 & 5 & 7 & 3 & 2 & 1 \\
\hline 10 & 10 & 10 & 7 & 3 & 2 \\
\hline 5 & 20 & 20 & 13 & 7 & 3 \\
\hline
\end{tabular}

Table 2.3: A priori uncertainty ( $\mathrm{Sv} \mathrm{rms}$ ) in the mass conservation equation for a single section. The results from the GCM are extrapolated at low latitudes.

baroclinic variability is potentially higher at low latitudes because of the fast baroclinic waves existing near the Equator, so that the variability in the wind field, which is associated with large Ekman transports, has a faster impact on the density field. Wijffels et al. (1996, Appendix B) used XBT time series to estimate baroclinic variability in the upper $400 \mathrm{~m}$ in the North Pacific Ocean at $10^{\circ} \mathrm{N}$ at roughly $\pm 7 \mathrm{~Sv}$, which agrees with my a priori value in the upper layers at $10^{\circ} \mathrm{N}$ (Table 2.3). However, it will be verified, a posteriori, that this extrapolation to low latitudes is adequate.

Table 2.3 was used to calculate the a priori uncertainties through each section, each layer and in each box. Those are listed in Appendix C, Table C-1 and C-2. In the case of outcropping layers, the uncertainty contribution from the section was made proportional to the actual length of the lower interface of the layer.

At $36^{\circ} \mathrm{N}$ in the Atlantic, the variability in the baroclinic silica fluxes was estimated to about $\pm 50 \mathrm{kmol} \mathrm{Sis}^{-1}$. The a priori uncertainty on the total silica conservation in a North Atlantic box between two sections is thus of $\sqrt{50^{2}+50^{2}+50^{2}}=86 \mathrm{kmol} \mathrm{Sis}^{-1}$, where the third term $\left( \pm 50 \mathrm{kmol} \mathrm{Si} \mathrm{s} \mathrm{s}^{-1}\right)$ accounts for net sources and sinks (discussed later in Section 4.3.1). The total a priori silica uncertainty in the North Atlantic was thus taken to $\Delta \mathrm{Si}^{\mathrm{N}}$. Atlantic $= \pm 100 \mathrm{kmol} \mathrm{Si}^{-1}$. This top-to-bottom silica uncertainty was extrapolated to other latitudes and oceans according to the average concentration of silica and the local mass variability $\Delta$ Mass $^{\text {local: }}$

$$
\Delta \mathrm{Si}^{\text {local }}=\Delta \mathrm{Si}^{36^{\circ} \mathrm{N}} \times \frac{\left\langle\mathrm{Si}^{\text {local }}\right\rangle}{\left\langle\mathrm{Si}^{36^{\circ} \mathrm{N}}\right\rangle} \times \frac{\Delta \text { Mass }^{\text {local }}}{\Delta \mathrm{Mass}^{36^{\circ} \mathrm{N}}},
$$

where $\Delta$ is the a priori uncertainty and $\langle\mathrm{Si}\rangle$ is the concentration. (For a given uncertainty 
in the baroclinic mass flux $\Delta$ Mass $^{\text {local }}$, it is assumed that a larger average silica concentration implies larger silica differences over the water column and thus a larger variability in the silica flux).

The a priori uncertainty in silica conservation equations in deep layers used by Macdonald (1998) was of the same order as the one obtained from variability, that is, 16 to $40 \mathrm{kmol} \mathrm{Si} \mathrm{s}^{-1}$. On the other hand, Robbins and Toole (1997) did not address oceanic variability and used a more stringent constraint for the silica budget in the Indian Ocean, ( $\pm 100 \mathrm{kmol} \mathrm{s}^{-1}$ compared with $\pm 700 \mathrm{kmols}^{-1}$ that is obtained from Eq.2.19 for a budget over the Indian Ocean, including the Pacific-Indonesian Throughflow section and the $32^{\circ} \mathrm{S}$ section).

Salinity and phosphate-oxygen "PO" concentrations do not vary greatly with location, and their uncertainties were derived from the uncertainty in the mass flux, as described in the anomaly equation formulation, Section 1.2.4 (page 22). 


\section{Chapter 3}

\section{Physical circulation}

The method described in Chapter 1 along with the a priori statistics from Chapter 2 (Section 2.6) are used to estimate the ocean circulation. In this chapter, the physical circulation is discussed individually for the Indian, Pacific, Atlantic and Southern Ocean with a focus on the meridional overturning circulation (MOC). (Here the expression "meridional overturning circulation" is used to denote the net zonally integrated stream function of the depth/meridional plane. There is no implication that it is restricted to a single top-to-bottom cell; the vertical structure is to be determined. Its strength will however, sometimes be measured by the net inflow of deep waters.) A summary of the global circulation follows, including global averages of dianeutral velocities and diffusivities. Each discussion starts with an introductory literature review (first section); it is followed by a presentation of the regional model constraints (second section), and a circulation description (third section). Global features of the freshwater divergences, heat, nutrient and oxygen fluxes are discussed in the following chapter.

The vertical structure of the net flux across the hydrographic sections is presented graphically as an overturning streamfunction which is defined as the cumulative sum of the transport in individual layers starting from the bottom. [This convention contrasts with some of the literature where the results are usually presented as individual layer

fluxes.] But it has the advantage of making the useful quantities readily accessible to the eye, the sum of the fluxes over several layers being just the difference of value of the streamfunction between the first and last layer. The direction of the flow is thus given by 
the slope with depth of the overturning streamfunction.

The results are compared with the existing literature. However, when the error bars were not provided by the author, the comparison remains qualitative. I assume that their error bar is zero, and qualify the number as "compatible" when inside my error bars and as "incompatible" when outside.

I start with a relatively extensive discussion about the circulation in the Indian Ocean as this work represents the first time that the high-quality WOCE data are used to estimate its basin-scale. Shorter presentations of the derived circulation in the Pacific, Atlantic and Southern Ocean follow, with a focus on basin-scale integrated transports.

A number of solutions were explored by changing the model configuration. The "standard", or "preferred" solution employs property anomalies and the constraints listed in Table 1.2 (page 17), that is, top-to-bottom silica is conserved as described in Section 2.6; salt is conserved top-to-bottom and within individual layers; heat, and the tracer combination "PO" $=170 \times \mathrm{PO}_{4}+\mathrm{O}_{2}$ (Redfield et al., 1963; Anderson and Sarmiento, 1994), are conserved only in layers below the surface. (The surface layer is non-conservative because of heat and oxygen exchanges with the atmosphere.) The freshwater flux was not constrained, but was solved for through the inversion. Again the a priori uncertainties are consistent with the error budget of Section 2.6, taking into account the oceanic circulation variability. Conservation equations for heat, salt and PO are written as anomaly equations, so that the mass divergence time the average property concentration is subtracted from the original conservation equation (see Chapter 1). Some constraints (e.g., "PO" in the South Atlantic) were given a lower weight when large mass residuals were obtained in the first-try solution. Alternative solutions are described in the discussion for each ocean.

Although water mass distributions guided my choice of the "first guess" circulation, the flow was not explicitly constrained to force the net layer fluxes to be advected away from their supposed sources. In several places, the estimated flow, although indistinguishable from zero within error bars, does not follow the large-scale property distributions in the sense suggested by Wüst. Away from the core of boundary currents, evidence is relatively weak that the large-scale property distributions of a time-dependent flow require the mean advective flux to follow the water mass pathways. Here, near-conservation on the large- 
scale is enforced, which is all that appears compelling to do when using such a model.

\subsection{The Indian Ocean}

\subsubsection{Introduction}

My discussion starts, somewhat arbitrarily, with the Indian Ocean. The Indian Ocean has at least four unique features compared to all other oceans: 1) a strong monsoonal variability; 2) a southward average Ekman transport on both sides of the Equator; 3) a low latitude northern boundary with 4) a net influx of warm water from the Indonesian Throughflow. This section is also the subject of a publication (Ganachaud et al., 1999) that is focused on the meridional overturning circulation.

Since the studies of Warren (1981) and Fu (1986), the meridional overturning circulation of the Indian Ocean has been the subject of a number of recent papers (Toole and Warren, 1993; Robbins and Toole, 1997 ; Lee and Marotzke, 1997, 1998; Macdonald, 1998; Zhang and Marotzke, 1999). Although the meridional overturning rate and structure are a basic descriptive feature of the general circulation as well as a determinant of the heat and other property fluxes, there remains significant disagreement both about its qualitative and quantitative features in the Indian Ocean.

From a hydrographic section at $18^{\circ} \mathrm{S}$, Warren (1981) estimated a northward flow of $19 \mathrm{~Sv}$ in the deep western boundary currents. $\mathrm{Fu}(1986)$, using the same section, but, combining it with four other sections in the Indian Ocean and doing a formal inverse calculation, found weak net deep water inflow of $3.6 \mathrm{~Sv}$, with the bulk of the net northward flow occurring at intermediate levels (1000-2000 decibars). In contrast, Macdonald (1998), again with the same $18^{\circ} \mathrm{S}$ section but combined with a global data set containing more recent sections than were available to $\mathrm{Fu}$, (notably at $32^{\circ} \mathrm{S}$ ), obtained $10 \mathrm{~Sv}$ of deep water northward flow at $18^{\circ} \mathrm{S}$ with a $5 \mathrm{~Sv}$ southward flow at intermediate levels.

Using the pre-WOCE 1987 section at $32^{\circ} \mathrm{S}$, and tracer properties to suggest a zerovelocity surface, Toole and Warren (1993) estimated the surprisingly large bottom water flux below 2000 decibars of $27 \pm 10 \mathrm{~Sv}$ geostrophically. Robbins and Toole (1997) recognized that this large flux would cause problems with the silica budget, and added silica 
conservation as a constraint. They obtained, through an inverse calculation, a weaker deep influx of $12 \pm 3 \mathrm{~Sv}$. At the two standard deviation level, this value is inconsistent with the non-silica-conserving Toole and Warren (1993)-type circulation, $29 \pm 5 \mathrm{~Sv}$, according to the Robbins and Toole (1997) error analysis. Assuming the error estimates are appropriately computed, the inconsistency implies systematic (non-random) errors present in the models. In both circulations, the intermediate waters (1000-2000 decibars) moved southward. Bryden and Beal (personal communication, 1999) showed that the inferred overturning was sensitive to the assumed strength of the Agulhas Current, and notably to the presence of a northward flowing undercurrent as detected by ADCP measurements.

Lee and Marotzke $(1997,1998)$, using a GCM, climatological hydrography and surface fluxes, inferred a mean northward overturning flow of $14 \mathrm{~Sv}$ in the upper $1000 \mathrm{~m}$ with a weak south-to-north deep water flow. Their solution is consistent with several other GCM calculations that exhibit weak deep inflow, but a vigorous shallow overturning (see Zhang and Marotzke, 1999, for a review). Such a shallow overturning conflicts with the direction of the flow as would be suggested by a classical water mass analysis (e.g., Toole and Warren, 1993).

\subsubsection{Indian Ocean Problems}

The Indian Ocean is complex, and a full discussion of the details of the circulation as previously described would be very lengthy. Here I only summarize some of the major uncertainties most directly affecting the inversion.

\section{Monsoonal Effects}

Because of the intense monsoonal shifts, estimation of the time-average circulation from synoptic sections is problematic; my main excuse for nonetheless doing so is that below the top few hundred meters, where my uncertainties are large, and away from the Somali Current, there is no evidence of a significant annual shift in hydrography or baroclinic flow

field (e.g., Warren and Johnson, 1992, Fieux et al., 1986). (Fieux et al. (1986) found a variation of $30 \%$ in the deep western boundary current transport at $10^{\circ} \mathrm{N}$, which may be due to internal wave noise (Section 2.3.1)). 
The most spectacular time-dependent feature is the establishment and reversal of the Somali Current whose net transport at the Equator ranges from 0 to $21 \mathrm{~Sv}$ (including undercurrents) (Schott et al., 1990). However, the GCM of Lee and Marotzke (1998) suggested that the upper ocean variability decreases toward the south (away from the Mozambique Channel). They estimated a monsoonal variability in the depth-latitude streamfunction of $\pm 20 \mathrm{~Sv}$ with associated changes in the meridional energy flux of $\pm 1.8 \mathrm{PW}$. But most of the streamfunction variations were explained by a barotropic response to Ekman transport variations, with a relatively small contribution from the vertical shear $( \pm 2 \mathrm{~Sv}$ or less). (The Somali Current region was exceptional; there the vertical shear contribution was large, of order $10 \mathrm{~Sv}$.) Barotropic fluctuations are not aliased in the hydrography so that a model using the mean Ekman flux can be combined consistently with sections taken at different seasons. In the present analysis, internal waves and variability in the baroclinic field are accounted for in the uncertainties (Chapter 2).

Non-linear interactions of the time-dependent depth-independent transports could conceivably generate significant rectified mean flows. Lee and Marotzke (1998) could, however, find no such effect in the energy.

\section{Agulhas Current and Mozambique Channel}

The gyral subtropical circulation is closed by the Agulhas Current, believed to transport between 66 and $70 \mathrm{~Sv}$ (Beal and Bryden, 1997). Stramma and Lutjeharms (1997) suggest that most of the Agulhas waters are fed by the subtropical gyre (e.g. their Figure 7), but with a fraction (20-25 Sv) from either the Mozambique Channel or the East Madagascar Current. Of these Stramma and Lutjeharms (1997) and Sætre and Da Silva (1984) suggested the latter was the more important, in contrast with Lutjeharms (1976) who concluded that the Mozambique Current is the major source of Agulhas waters. Sæetre and Da Silva (1984) found no continuous upper ocean current in the Mozambique Channel and little contribution to the Agulhas Current. This inference was in agreement with the study of Ménaché (1963) who believed that the Mozambique Current curved eastward and flowed back to the north along the west coast of Madagascar. Recent CTD data and satellite imagery showed that the Agulhas Current is partly fed by the Mozambique Current, and 
partly by a westward flow south of Madagascar (Gründlingh, 1993).

Fu's (1986) inverse box model produced a net southward flux of $6 \mathrm{~Sv}$ in the Mozambique Channel, similar to that of Stramma and Lutjeharms (1997). The global inversion of Macdonald (1998) did not derive a significant flux there.

The flow through the Mozambique Channel appears to be highly variable on seasonal time scales. Measurements from tide gauges combined with hydrography (Donguy and Piton, 1991) show an annual cycle with \pm 10 Sv amplitude in the Channel. Altimetric data (not shown) examined by Marotzke (personal communication, 1999) show fluctuations of $\pm 25 \mathrm{~cm}$ in the sea surface height difference between South Madagascar and Africa $\left(25^{\circ} \mathrm{S}\right)$. Assuming a linear decrease of the velocity down to 1000 decibars, the implied variations in the net geostrophic transport are $\pm 20 \mathrm{~Sv}$ (This calculation gives only an order of magnitude estimate, but the 1000 decibar vertical scale and the linear decrease are consistent with the solution described below. Baroclinic Kelvin waves may account for these rapid fluctuations. Seasonal variability is probably also present in the density field, as observed in the GCM of Lee and Marotzke (1998) in the Somali region.)

\section{Pacific-Indonesian Throughflow (PIT)}

The Pacific-Indonesian Throughflow (hereafter PIT) is highly variable and poorly sampled, but a potentially important element of the Indian and Pacific oceans circulation. If the PIT average transport were $10 \mathrm{~Sv}$ at $20^{\circ} \mathrm{C}$ and assuming that it ultimately leaves the Indian Ocean at $10^{\circ} \mathrm{C}$, it would produce a convergence of $0.4 \mathrm{PW}$ in the Indian Ocean heat transport budget. This flux convergence is of the same size as the total climatological heat input to the Indian Ocean by air-sea exchanges from Oberhuber (1988).

The published estimates of the net PIT mass transport range from $18 \pm 7 \mathrm{~Sv}$ westward to $2.6 \mathrm{~Sv}$ eastward (Fieux et al., 1996), with a host of estimates between these two extremes, depending upon the measurement time and method (Godfrey, 1996). Macdonald (1998) constrained her inverse model to a net PIT of $10 \pm 10 \mathrm{~Sv}$ and obtained, after inversion, a flux of $9 \pm 7 \mathrm{~Sv}$. If not specifically constrained, her model produced a net PIT of $11 \pm 14 \mathrm{~Sv}$. Interestingly, her sensitivity experiments $(\mathrm{PIT}=0 \mathrm{~Sv} ; \mathrm{PIT}=20 \mathrm{~Sv})$ also showed that variations in the PIT strength had no effect on her estimated Agulhas Current and 
Drake Passage fluxes. Nevertheless, such changes affect dramatically property budgets and derived air-sea exchanges in the Indian and Pacific oceans. Zhang and Marotzke (1999) estimated the PIT from climatological Indian Ocean hydrography and a GCM, and found that a small mean inflow $(2.7 \mathrm{~Sv})$ was sufficient to close the Indian Ocean heat and salt balance.

The strength of the PIT is plausibly dependent upon the phase of ENSO (e.g., Wajsowicz, 1994, 1997, who used a numerical model). From XBT time series and historical data, Meyers et al. (1995) found a total mean transport in the upper $400 \mathrm{~m}$ of $5 \mathrm{~Sv}$ to the west with a $\pm 2.5 \mathrm{~Sv}$ variation in the annual cycle. They estimated a top-to-bottom mean transport of $7 \mathrm{~Sv}$. Moorings in the Makassar Strait, which is believed to be the main path of the Throughflow, showed an annual average transport of $9 \mathrm{~Sv}$ in 1997 with variations from $5 \mathrm{~Sv}$ during El Niño to $12 \mathrm{~Sv}$ during La Niña (Gordon and Susanto, 1998). For present purposes, I use a Throughflow of $7 \pm 7 \mathrm{~Sv}$ as the initial best estimate. (But to account for variability and measurement noise from my error budget, the actual constraint on the PIT between Indonesia and Australia is $7 \pm 15 \mathrm{~Sv}$, as explained below.)

\subsubsection{Model constraints}

Figure 3-1 shows the Indian Ocean sector of the model which is made of the WOCE sections I2 (Dec. 1995-Jan. 1996, e.g., Johnson et al., 1998); I3 (Apr.-June 1995); I4 (June 1995); I10 (Nov.-Dec. 1995, Bray et al. (1997); the pre-WOCE section I5 (Nov. 1987; Toole and Warren, 1993; Robbins and Toole, 1997) and the Java Australia Dynamics Experiment (JADE) section (Aug. 1989; Fieux et al., 1994; Coatanoan et al., 1999). (The 1989 JADE data were used instead of the 1992 data because they included nutrient measurements. However, the 1989 data were combined with a station of the 1992 data to complete the section, as discussed below (Section 3.1.6.)) I2, I3 and I4 were obtained from the WHPO data center with permission of the respective PIs B. Warren, G. Johnson, W. Nowlin and

J. Toole. I10 was provided by G. Packard with permission of the PIs J. Toole, N. Bray and J. Sprintall. The data from the Franco-Indonesian JADE cooperation were provided by M. Fieux and A. G. Ilahude (PIs); B. Coste and C. Coatanoan (nutrients).

Some unrealistically large geostrophic velocities were found in the thermocline waters 
of 12 due to narrow station spacing and small Coriolis parameter. An attempt was made to apply a horizontal filter to the temperature and salinity fields in the thermocline waters. The filter wavenumber cut-off was set so that large velocities $(>2 \mathrm{~m} / \mathrm{s})$ were removed. The filtering did not change the integrated mass transports so I decided to leave the original temperature and salinity fields to avoid the arbitrary treatment of edges in the filtering process. Some velocities are unrealistic when station spacing is narrow, but the implied transport is accounted for in the error budget.

Constrained boxes are defined for the Indian Ocean as the "subtropical box" (I5, I4, I3), the "Mozambique Channel box" (I4, I2W), the "Tropical box" (I3, I2, I10) and the "North Indian box" (I2W, I2, I10, J89). Note that the North Indian box includes the area between $\mathrm{I} 10$ and J89 because the I10 section could not be completed up to the Indonesian coast. The layers, defined by neutral surfaces (Table 3.1), were chosen to facilitate the comparison with the existing literature, to match the Southern Ocean layers of the global model, and to have a relatively homogeneous thickness over the basin. Mass conservation was required consistent with the error budget of Chapter 2 (see Table C-2, page 194). The resulting a priori uncertainties that characterize the net transports range from $\pm 7 \mathrm{~Sv}$ at $32^{\circ} \mathrm{S}$ to $\pm 15 \mathrm{~Sv}$ on the JADE 89 PIT section. The Ekman transports from the NCEP reanalysis (Kalnay et al., 1996) with their a priori uncertainties are listed in Table 3.2.

Because of the large baroclinic variability in the East African Coastal Current (EACC) on section I2W, a large adjustment $( \pm 20 \mathrm{~Sv})$ was allowed to the flow in the first layer.

The Indian Ocean topography is complex (Figure 3-1) and the deep layer fluxes were broken down and constrained according to the bathymetric features. These "bathymetric constraints" are listed in Table 3.3. Unlike the RT 97 inversion, the net bottom water flow into the Crozet and Perth Basins is not constrained to be northward. This change is discussed further below. The flux into the Mozambique basin was weakly constrained to be northward ( $1 \pm 2 \mathrm{~Sv}$ ). The flux below 1300 decibars in the PIT was constrained to be small as this level corresponds to the limit of the waters of Indonesian origins (Fieux et al., 1994). 


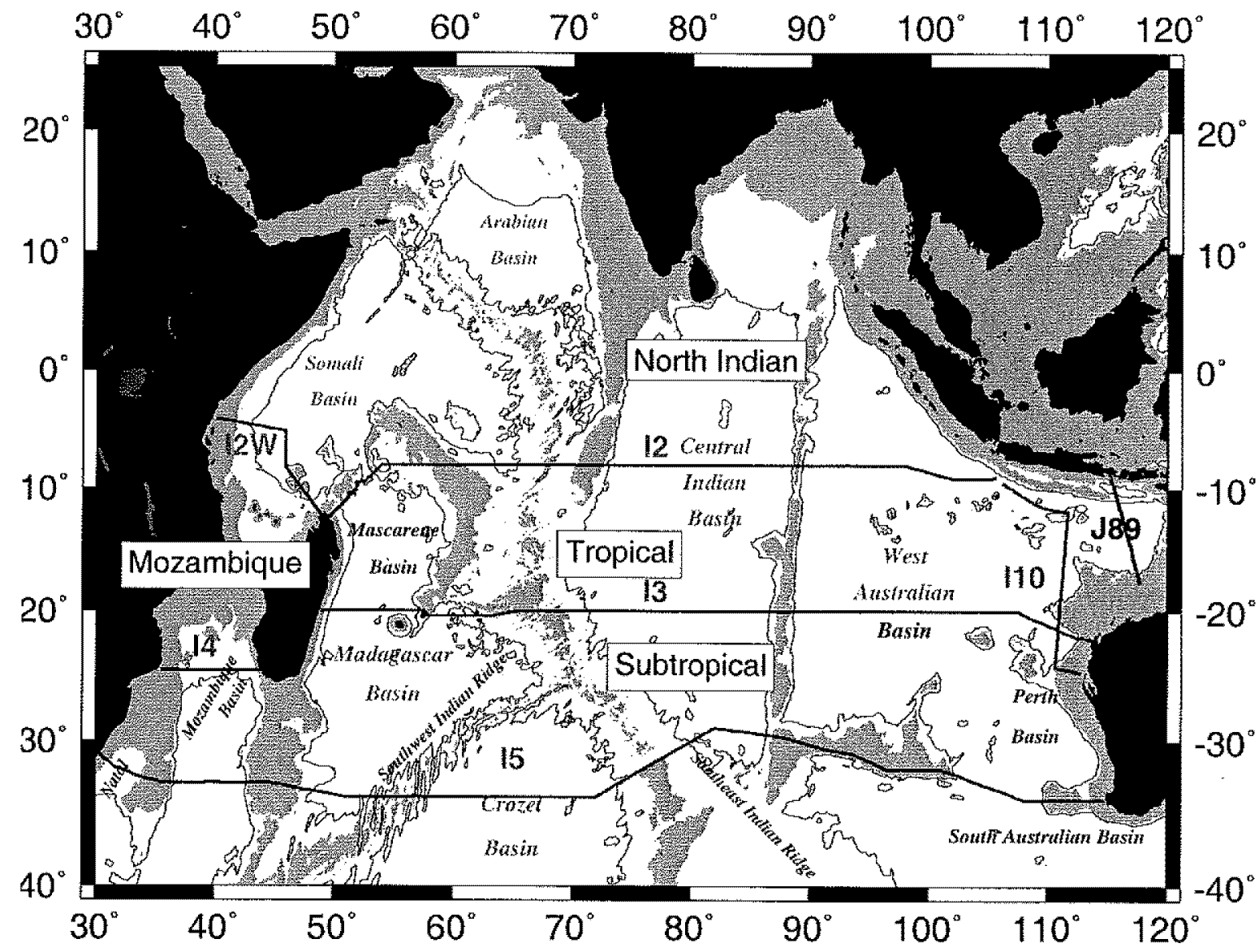

Figure 3-1: Indian Ocean sections and topography. The $4000 \mathrm{~m}$ isobath is contoured and areas shallower than $3000 \mathrm{~m}$ are shaded. Box names are indicated in the shaded rectangles. 


\begin{tabular}{|c|c|c|c|c|}
\hline Layer & $\begin{array}{l}\text { Upper } \\
\text { Interface }\end{array}$ & $\begin{array}{l}\text { RT97 } \\
\text { Layer }\end{array}$ & $\begin{array}{l}\text { I5 Interface Property } \\
\text { from RT97 }\end{array}$ & $\begin{array}{l}\text { Water Mass } \\
\text { Name }\end{array}$ \\
\hline 1 & Surface & \multirow[t]{2}{*}{1} & & \multirow[t]{2}{*}{ Surface Water } \\
\hline 2 & $\gamma^{n}=25$ & & & \\
\hline 3 & $\gamma^{n}=26.2$ & 2 & & Subantarctic Mode Water \\
\hline 4 & $\gamma^{n}=26.9$ & 3 & $\sigma_{\theta}=26.80 ; \theta=9.85$ & Upper Antarctic Mode Water \\
\hline 5 & $\gamma^{n}=27.36$ & 4 & $\sigma_{\theta}=27.20 ; \theta=4.65$ & Lower Antarctic Mode Water \\
\hline 6 & $\gamma^{n}=27.7$ & 5 & $\sigma_{2}=36.65 ; \theta=3.26$ & Upper Deep Water \\
\hline 7 & $\gamma^{n}=27.96$ & \multirow[t]{2}{*}{6} & \multirow[t]{2}{*}{$\sigma_{2}=36.92 ; \theta=2.20$} & \multirow{2}{*}{ Lower Deep Water } \\
\hline 8 & $\gamma^{n}=28.07$ & & & \\
\hline 9 & $\gamma^{n}=28.11$ & \multirow[t]{2}{*}{7} & \multirow[t]{2}{*}{$\sigma_{4}=45.89 ; \theta=1.28$} & \multirow[t]{2}{*}{ Upper Bottom Water } \\
\hline 10 & $\gamma^{n}=28.15$ & & & \\
\hline 11 & $\gamma^{n}=28.23$ & 8 & $\sigma_{4}=46.01 ; \theta=0.43$ & Lower Bottom Water \\
\hline
\end{tabular}

Table 3.1: Indian Ocean layers $\left(\gamma^{n}\right.$ upper interface in $\left.\mathrm{kg} \mathrm{m}^{-3}\right)$. For comparison, the Robbins and Toole (1997) layers are indicated along with the potential density and temperatures at each interface (from their table 1). The italicized layer numbers separated by the line indicate a slightly different interface $\left(\gamma^{n}=26.20\right.$ instead of 26.50 in RT97).

\begin{tabular}{|c|c|c|c|}
\hline Section & Initial & Final & $\%$ correction \\
\hline I5 & $0.3 \pm 1$ & $0.5 \pm 1$ & - \\
\hline I4 & $-0.5 \pm 1$ & $-0.5 \pm 1$ & - \\
\hline I3 & $-11.5 \pm 5$ & $-10 \pm 4$ & $-8 \%$ \\
\hline I2W & $-4.5 \pm 20$ & $9 \pm 10$ & $-300 \%$ \\
\hline I2 & $-11.6 \pm 5$ & $-11 \pm 5$ & $-6 \%$ \\
\hline I10 & $-2.9 \pm 1$ & $-2.9 \pm 1$ & $0 \%$ \\
\hline J89 & $-0.65 \pm 1$ & $-0.6 \pm 1$ & - \\
\hline
\end{tabular}

Table 3.2: Initial Ekman transport and that derived from the model (Final). The adjustments apply to the total transport in the first layers, including in principle any deviation from the time-mean geostrophic transport (e.g., monsoonal variability). The relative amplitude of the adjustment is indicated for transports larger than $1 \mathrm{~Sv}$. 


\begin{tabular}{|c|c|c|c|c|}
\hline Name & Section & Layer Depth & Net Flux & Longitude \\
\hline Natal Valley & 15 & $9-10(3400)$ & $0 \pm 0.5$ & $30^{\circ} \mathrm{E}-34^{\circ} \mathrm{E}$ \\
\hline Mozamb. B. & $\mathrm{I} 5$ & $9-11(3500)$ & $1 \pm 2$ & $35^{\circ} \mathrm{E}-46^{\circ} \mathrm{E}$ \\
\hline Madagascar B. & $\mathrm{I} 5$ & $9-11(3600)$ & $0 \pm 5$ & $46^{\circ} \mathrm{E}-56^{\circ} \mathrm{E}$ \\
\hline Central B. & 15 & $9-11(4000)$ & $0 \pm 1$ & $78^{\circ} \mathrm{E}-89^{\circ} \mathrm{E}$ \\
\hline Mozamb. Sill depth & I4 & $8-11(2800)$ & $0 \pm 0.5$ & Full section \\
\hline $\begin{array}{r}\text { PIT sill, mass } \\
\text { silica }\end{array}$ & J89 & $\begin{array}{l}6-10(1300) \\
\text { each layer } \\
\text { total } \\
\text { each layer }\end{array}$ & $\begin{array}{l}0 \pm 6 \\
0 \pm 3 \\
0 \pm 140 \\
0 \pm 70\end{array}$ & $\begin{array}{l}\text { Full section } \\
\text { kmol Si s }^{-1} \\
\text { kmol Si s }^{-1}\end{array}$ \\
\hline Name & Sections & Layer Depth & Net divergence & Longitude \\
\hline Mozamb. B. & $15 / 14$ & $8-11(2800)$ & $0 \pm 2$ & $35^{\circ} \mathrm{E}-46^{\circ} \mathrm{E} / \mathrm{Fulll} \mathrm{I} 4$ \\
\hline Perth Basin & I5/I3 & $9-11(3500)$ & $0 \pm 4$ & $96^{\circ} \mathrm{E}$-end $/ 87^{\circ} \mathrm{E}$-end \\
\hline Mascarene B. & $\mathrm{I} 3 / \mathrm{I} 2$ & $8-11(3000)$ & $0 \pm 4$ & $49^{\circ} \mathrm{E}-58^{\circ} \mathrm{E} / 49^{\circ} \mathrm{E}-59^{\circ} \mathrm{E}$ \\
\hline West Aust. B. & $\mathrm{I} 3 / \mathrm{I} 2 \mathrm{I} 10$ & $8-11(3000)$ & $0 \pm 4$ & $96^{\circ}$ E-end $/ 89^{\circ} \mathrm{E}$-end \\
\hline
\end{tabular}

Table 3.3: "Bathymetric" constraints on the flux in layers. The constraints are imposed on either the total flux through a single section (upper part, positive northward) or on the divergence between two sections (lower part). The layer and depth below which the flux is constrained are indicated along with the uncertainty on the net flux (single section) or divergence (between sections) (in Sv). "B." denotes "basin".

\section{Reference Surfaces and Dianeutral terms}

The initial zero-velocity-surfaces, or "reference surfaces", are indicated in Table 3.4. The I5 reference surface was taken from the RT97/TW93 initial guess with a modification close to the coast in the Agulhas Current region to account for the presence of an Agulhas Undercurrent (Beal and Bryden, 1997, Fig. 3 ). In general, a deep reference surface was preferred to a shallow one to decrease the potentially large velocity errors at depth, which can result in an eddy-induced shear region. The northward moving bottom waters in the abyssal Indian Ocean lie in the potential temperature range -0.8 to $1.4^{\circ} \mathrm{C}$ in the western basin and 0 to $1^{\circ} \mathrm{C}$ in the eastern basin (Schmitz, 1996b, p.122). Therefore, the interface between the Circumpolar Deep Water and the "Indian Ocean Deep Water" defined by $\gamma^{n}=28.11\left(\theta=1.2^{\circ}\right.$ at $\left.20^{\circ} \mathrm{S}\right)$ was chosen as a "first guess" for the I2, I3 and I10 reference surface. (a similar surface was chosen by other authors, e.g. Johnson et al., (1998, Table 1.)) The model is not crucially dependent upon this choice: an experiment which started with a bottom reference surface at I2, I3, I10 and I5 (away from the Ag- 


\begin{tabular}{|l|l|l|}
\hline Section & Reference Surface & Remark \\
\hline \hline I5 & station-specific & TW93/Beal and Bryden $(1997)$ \\
\hline I4 & $2500 \mathrm{db}$ & Mozambique sill depth \\
\hline I2W & bottom & See text \\
\hline I2,I3,I10 & $\gamma^{n}=28.11$ & RT97/TW93 \\
\hline J89 & bottom & See text \\
\hline
\end{tabular}

Table 3.4: Initial zero-velocity-surface, or reference surfaces.

ulhas Undercurrent) produced a similar overturning after inversion. Strong shear down to 3000 decibars in the composite JADE section led to the choice of a bottom reference level, with the bathymetric constraint ensuring no net flow in individual layers below the sill depth $\left(\gamma^{n}=27.7,1300\right.$ decibars $)$ by adjusting the reference velocities accordingly.

In common with previous inverse solutions, the small scales of the reference velocity $\left(b_{i}\right)$ are not resolved, and the posterior uncertainties on the individual $b_{i}$ are almost unchanged from the prior values. Following the error budget (Section 2.5), the a priori uncertainties were given a minimum value of $\pm 1 \mathrm{~cm} \mathrm{~s}^{-1}$ and increased according to the local shear and when current-meter data suggested it (Dickson, 1989, page E-9, E-11 ; Schott et al., 1988).

A large range of adjustment was allowed to dianeutral transfers $w^{*}\left( \pm 10^{-3} \mathrm{~cm} \mathrm{~s}^{-1}\right)$ and mixing coefficients $\kappa^{*}\left( \pm 100 \mathrm{~cm}^{2} \mathrm{~s}^{-1}\right)$. These a priori values are near the upper limit of, or larger than, published estimates (e.g. TW93, RT97; Polzin et al., 1997; Munk and Wunsch, 1998), leaving the model free to determine the coefficients from the constraints. However, both upwelling and mixing turn out to be well-determined by heat and salt anomaly conservation requirements, and the final values have smaller amplitudes than the a priori ranges. No positivity requirement was set for $\kappa^{*}$.

\subsubsection{Indian Ocean Circulation: standard solution}

The resolution matrix of the Gauss-Markov estimate shows that heat and salt anomaly conservation in the layers are well resolved, i.e., provide independent information. The anomaly equations for the conservative tracer "NO" $\left(9.1 \times \mathrm{NO}_{3}+\mathrm{O}_{2}\right.$; Broecker (1974)) are, in general, indistinguishable form the "PO" equations and I choose to conserve "PO" because the nitrogen cycle is the more complex one. Mass conservation equations are only 
a) Subtropical $32 \mathrm{~S}-20 \mathrm{~S}$

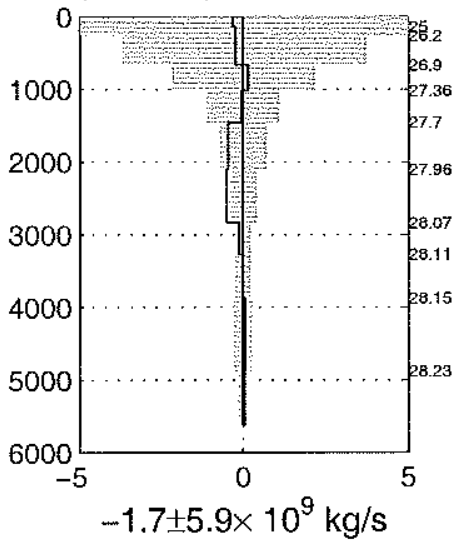

b) Tropical $20 \mathrm{~S}-8 \mathrm{~S}$

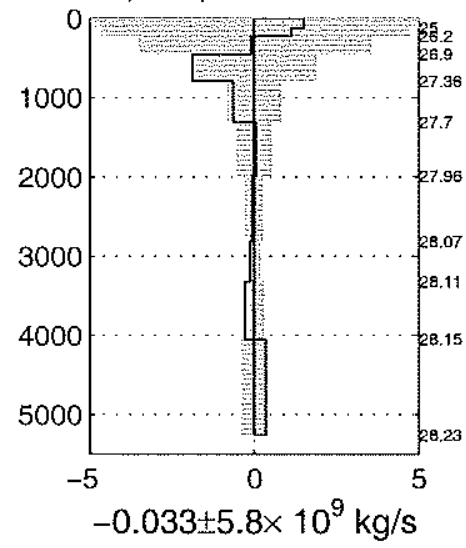

c) North (8S-JADE 89-Coast)

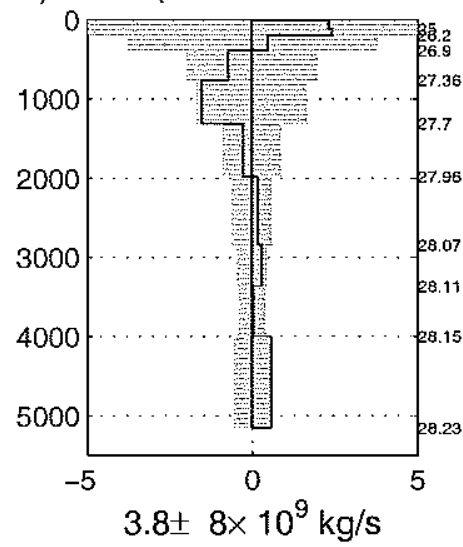

Figure 3-2: Standard solution mass residuals in the Indian Ocean in a) the subtropical box; b) the tropical box; and c) the North Indian box, which also includes the area between I10 and JADE 89. The line gives the residual in invidual layers (in $10^{9} \mathrm{~kg} \mathrm{~s}^{-1}$ ) and the shaded area give the one standard deviation uncertainty. The top-to-bottom residual is indicated below the graph.

weakly resolved. The solution elements (reference velocities; Ekman transport; freshwater flux; dianeutral mass transfers and mixing) were all within the a priori range. Except for silica, all constraints were met within uncertainties, and the solution appears to be both dynamically and statistically acceptable. Dissolved silica in the Indian Basin is conserved only at two standard deviations, with a net convergence of $-620 \pm 360 \mathrm{kmol} \mathrm{Si} \mathrm{s}^{-1}$, possibly associated with the high PIT flux (there is an equivalent divergence in the central Pacific, see Section 4.3.1). Mass residuals in individual layers are smaller than $1 \mathrm{~Sv}$ and all are indistinguishable from zero within two standard deviations (Fig. 3-2). The net residual in the Mozambique box (not shown) is also small at $0 \pm 7 \mathrm{~Sv}$. The I3 and I10 intersection near Australia (Fig. 3-1) generates a small closed box. Experience shows such boxes should not be constrained, owing to the potentially large hydrographic aliases at open-ocean intersections. Nevertheless, the residuals in this small area were confirmed, a posteriori, to be small.

Small adjustments were made to the initial NCEP Ekman transports (0\% to 8\%, Table 3.2), except in I2W (300\%) in the Mozambique Channel where the variability in the baroclinic transports is large. (The adjustment is not attributable solely to the Ekman transport, as it includes everything accommodated in the first layer(s) to meet the con- 
servation constraints.)

The reference velocities, absolute velocitics and transports cumulated from West (or from North along JADE 89, I2W and I10) from the standard solution are given in Appendix D (pages 196-209) but my discussion will focus on the net integrated fluxes and divergences.

\section{Net Fluxes}

The integrated flow for the standard solution, or net "overturning streamfunction" and the net flux across each section are shown on Figure 3-3. (Again, overturning streamfunctions are used, the slope of the curve indicating the direction of the flow, positive to the north for zonal sections, and to the east for JADE). The uncertainties, calculated from the error covariance of the solution vector, are given by the shaded area.

The net meridional mass fluxes, which include the Ekman transports, are interpreted as equal to the PIT flux and are given below each graph of Figure 3-3. These net mass flux estimates are the same, across each latitude within error bars. At $32^{\circ} \mathrm{S}$, the PIT flux is estimated as $16 \pm 5 \mathrm{~Sv}$ (Fig. 3-3a). Through J89, the net flux is $15 \pm 3 \mathrm{~Sv}$ westward (Fig. 33f), in the upper range of the a priori constraint on the PIT. (The final uncertainty of the PIT is surprisingly small, given the prior uncertainty. Its size results from the bathymetric constraints below the sill depth (Table 3.3). An uncertainty of $\pm 5 \mathrm{~Sv}$ is believed to be more realistic, as discussed later). In the Mozambique Channel, the flux is southward and of the same magnitude as the PIT, $14 \pm 6 \mathrm{~Sv}$ (I4 and I2W, Fig. 3-3d, e), implying a weak and uncertain net transport between Madagascar and Australia of $-4 \pm 8 \mathrm{~Sv}$ (I3, not shown). The net transport through $\mathrm{I} 10$ is westward but very uncertain, $2 \pm 14 \mathrm{~Sv}$, so that the mass flux between Java and the I2-I10 intersection is not known.

\section{Deep Inflow}

The estimated Indian Ocean overturning, or deep inflow below 2000 dbars at $32^{\circ} \mathrm{S}$ is $10.6 \pm 4 \mathrm{~Sv}$ (I5, Fig.3-3a). Similar values are obtained at $20^{\circ} \mathrm{S}$ (i.e., $\mathrm{I} 4+\mathrm{I} 3$ ) and $8^{\circ} \mathrm{S}$ $(\mathrm{I} 2 \mathrm{~W}+\mathrm{I} 2+\mathrm{I} 10)$, although the vertical structure changes due to dianeutral exchanges (described below). The net overturning (i.e., deep inflow) is about the same magnitude as 
a) 15 (32S)

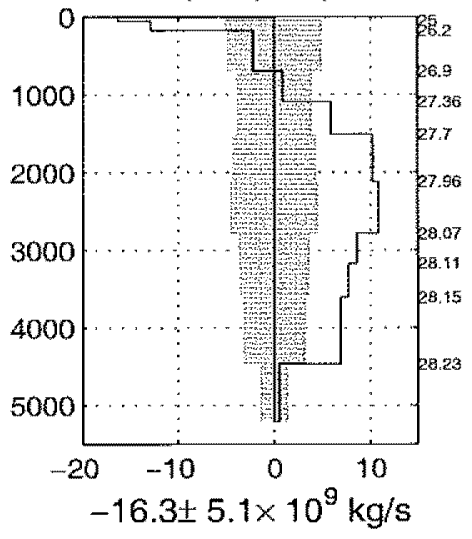

d) 14 (Mozambique S)

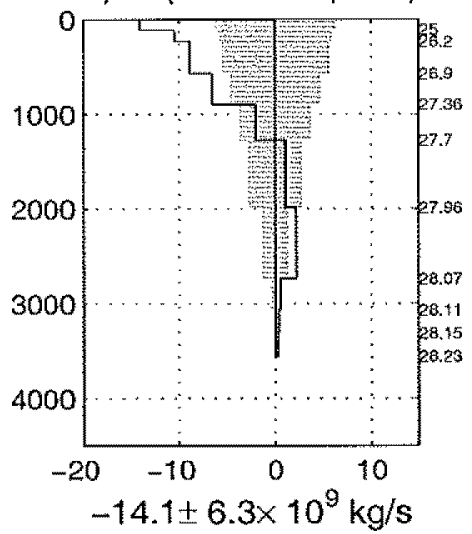

b) $13+14(20 S)$

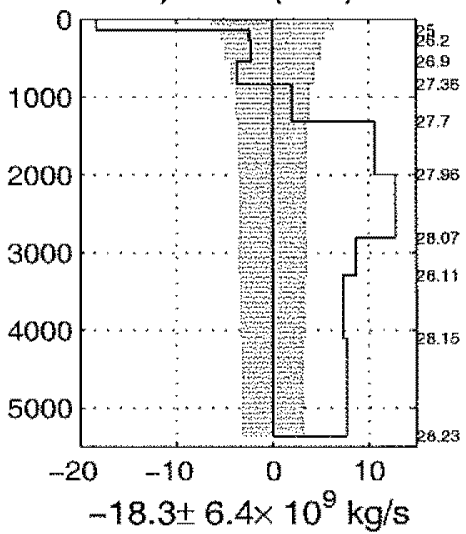

e) I2W (Mozambique N)

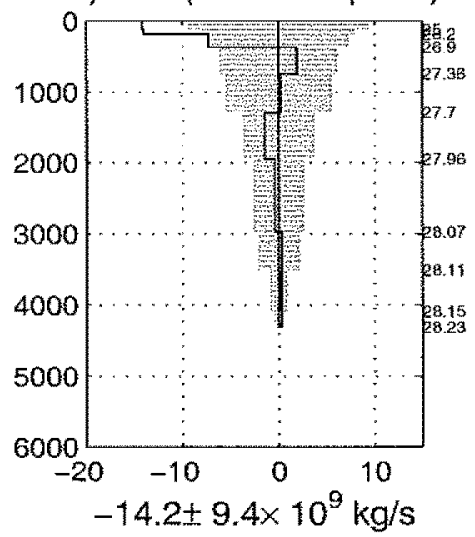

c) $12 W+12+110(8 S)$

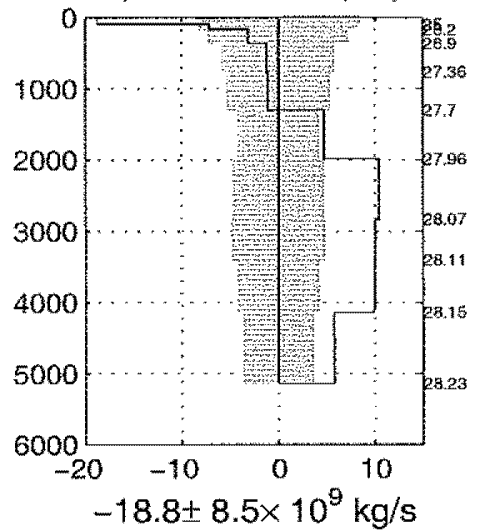

f) JADE 89 (116E)

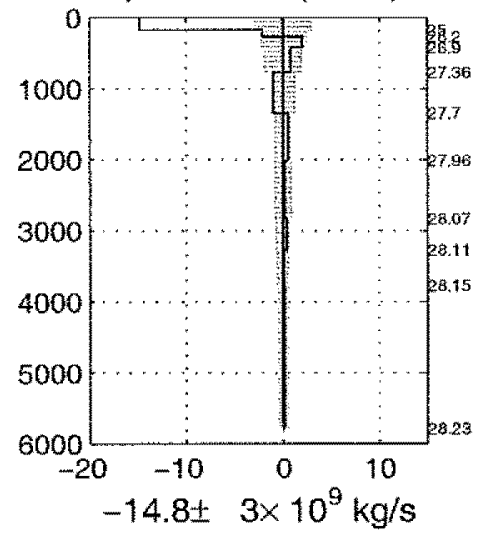

Figure 3-3: Overturning streamfunction for the standard solution across latitudes 2$) 32^{\circ} \mathrm{S}$; b) $20^{\circ} \mathrm{S}$; c) $8^{\circ} \mathrm{S}$; d) across the southern and e) northern Mozambique Channel; f) across the PIT. Each step of the streamfunction gives the direction and magnitude of the flow in the layer above (positive to the north/east). The layer interfaces are indicated to the right of each graph. 
the one obtained by RT97, the flow has a different structure. In my solution, the Agulhas Undercurrent carries $13 \pm 5 \mathrm{~Sv}$ to the north in layers 6 to 7 west of $31^{\circ} \mathrm{E}$ with an immediate southward recirculation to its east, producing an insignificant transport of $3 \pm 6 \mathrm{~Sv}$ to the north between the coast of Africa and $35^{\circ} \mathrm{E}$ in those layers. This feature was not present in the previous inversions. Due to the lack of resolution of the model short scales, the horizontal partition of the flow is not accurately known. However, in the Madagascar-Crozet basin and below 2000 dbars, there is no indication of an anticyclonic circulation as in RT97 (their Fig. 3), but a quasi-uniform northward flow east of $52^{\circ} \mathrm{E}$ (Fig. D-2, page 197). In the Perth basin, there is a weak and uncertain anticyclonic circulation and the net flux below 2000 dbars is indistinguishable from zero ( $1 \pm 8 \mathrm{~Sv}$ to the north).

The vertical flow structure differs with the RT97 as well (Fig. 3-3). Below $\gamma^{n}=28.11$, my northward flux is of $8 \pm 3.5 \mathrm{~Sv}$, whereas the deep inflow in the RT97 solution below this surface was $12 \pm 3 \mathrm{~Sv}$. But in my standard solution there is $2.6 \mathrm{~Sv}$ of northward flow just above $\gamma^{n}=28.11$, so that the total deep inflow below 2000 dbars is similar to the that of RT97. RT97 constrained the deep flow to move away from its sources in individual basins, resulting in an equivalent constraint on the Circumpolar Deep Water (CDW) flux of $18 \pm 5 \mathrm{~Sv}$ below $\gamma^{n}=28.11$ (their Table 3 , with the errors computed by assuming the constraints in the different basins are independent). The magnitudes and weights of these constraints were imposed subjectively (P. Robbins, personal communication, 1998) and account for their larger resultant inflow at depths.

At $32^{\circ} \mathrm{S}$, but in the bottom layers alone, (layers 9-11, 3200 dbars to bottom, not shown) the flux indicates northward-directed boundary currents along the ridges. The net flux across the Indian basin and below $3200 \mathrm{dbars}$ is $8 \pm 4 \mathrm{~Sv}$ to the north. Half of this occurs in the Madagascar-Crozet basins while the other half occurs in the Perth Basin. But the character of the horizontal structure of the deep flow is uncertain dependent upon depth.

At $20^{\circ} \mathrm{S}$ between Madagascar and Australia, the flux in layers below 2000 dbars, $11 \pm$ $4 \mathrm{~Sv}$, occurs mainly in the Mascarene Basin (Fig. D-6, $7 \pm 6 \mathrm{~Sv}$ northward-of which $4 \pm 5 \mathrm{~Sv}$ occurs west of Mauritius $\left(57^{\circ} \mathrm{E}\right)$ ). In both the Central Indian and West Australian basins, the flows are noisy and uncertain (respectively $0.5 \pm 7 \mathrm{~Sv}$ and $3 \pm 7 \mathrm{~Sv}$ northward below 2000 dbars), reflecting again the lack of horizontal resolution. Farther north, the fluxes in 
individual basins become more and more uncertain (Figs D-8-D-12).

The total deep water inflow into the Indian Ocean, $10.6 \pm 4 \mathrm{~Sv}$ is thus consistent with the RT97 result (12 $\pm 3 \mathrm{~Sv})$ and larger than the GCM results. This overturning is the sum of larger local horizontal fluxes that partially cancel, e.g., those in the Agulhas Undercurrent; a 15-Sv eddy near $39^{\circ} \mathrm{E}$, and the Madagascar-Crozet Basin circulation (Fig. 197). The present model would produce an uncertainty of $\pm 8 \mathrm{~Sv}$ with total mass and silica conservation only (cf., RT97). Addition of the other sections and constraints (heat, layers, etc.) accounts for the reduced uncertainty.

\section{Intermediate and Surface Flows}

The cumulative flow from the west across $32^{\circ} \mathrm{S}$ and above 2000 dbars (layers 1-6) is shown in Fig. D-2(upper). In the surface layers, at $32^{\circ} \mathrm{S}$ (I5), the Agulhas Current carries $74 \pm 7 \mathrm{~Sv}$ to the south in layers 1 to 5 west of $32^{\circ} \mathrm{E}$, which is thus consistent with the Beal and Bryden (1997) estimate of $69 \pm 2 \mathrm{~Sv}$. No obvious horizontal recirculation is observed, consistent with the RT97 results; the gyre northward wind-driven flow occurs over the whole interior.

At $25^{\circ} \mathrm{S}$ in the Mozambique Channel (I4, Fig. D-4d), a strong anticyclonic circulation results in a powerful southward current of $23 \pm 3 \mathrm{~Sv}$ at the western boundary in layers 1 to 5 between $36^{\circ} \mathrm{E}$ and $37^{\circ} \mathrm{E}$.

\section{Dianeutral Transfers and Mixing}

Dianeutral mass transfers (Fig. 3-4 upper panels) indicate downwelling at the base of the surface layers of the subtropical and North Indian boxes. Most of the deep upwelling appears to take place in the North Indian box, with about $10 \pm 5 \mathrm{~Sv}\left(3 \pm 1.5 \times 10^{-5} \mathrm{~cm} \mathrm{~s}^{-1}\right)$. The deep dianeutral transfers in the subtropical and tropical boxes are not significantly different from zero. The upwelling in the North is associated net dianeutral mixing equivalent to diffusivity values of 4 to $10 \mathrm{~cm}^{2} \mathrm{~s}^{-1}$ with larger values in the deepest layer approaching $30 \mathrm{~cm}^{2} \mathrm{~s}^{-1}$. (Owing to possible large errors in the horizontal area of neutral surfaces at depths, this latter value is very uncertain). In this box, downgradient mixing is found consistently over all the deep interfaces, although there was no requirement that the mix- 
a) Subtropical $32 S-20 S$
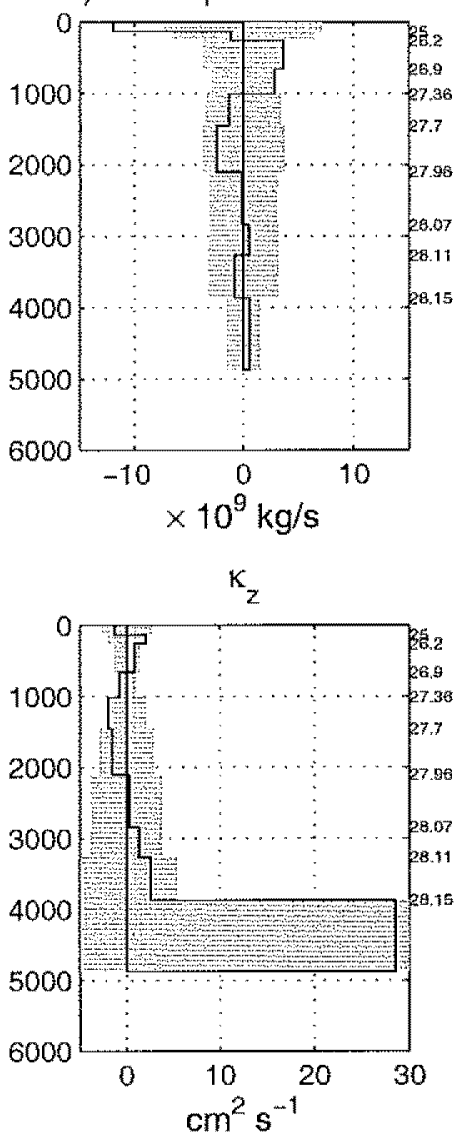

b) Tropical 20S-8S
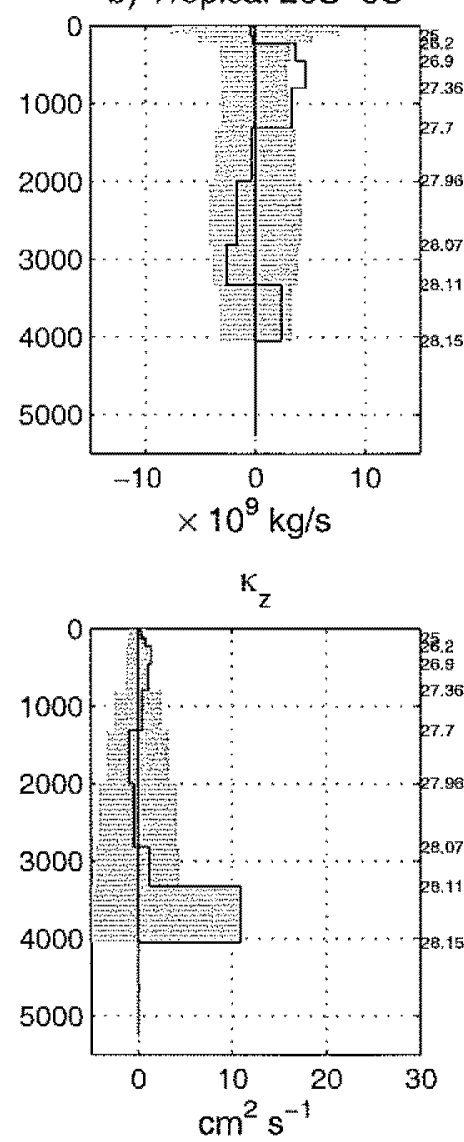

c) North (8S-JADE 89-Coast)
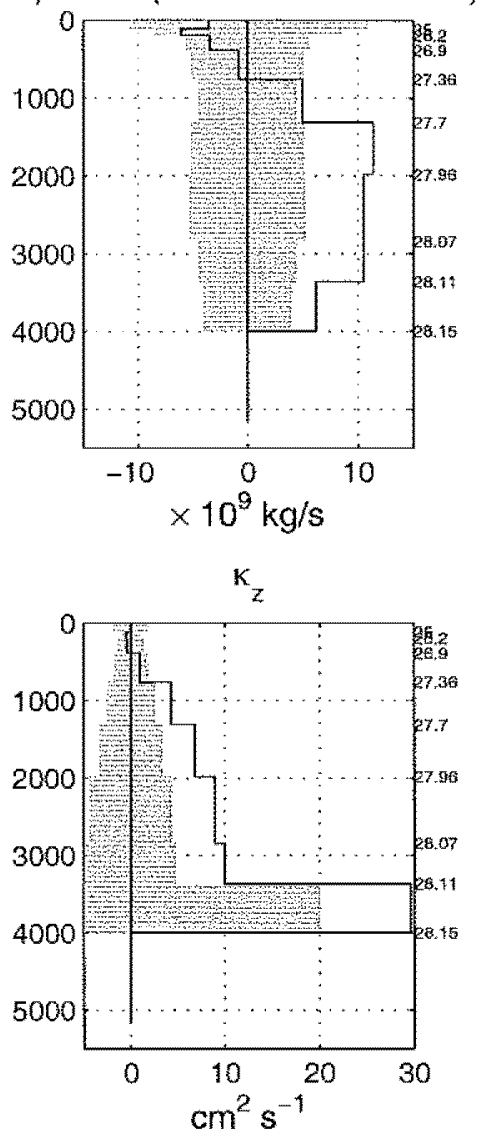

Figure 3-4: Dianeutral transfers (upper) and diffusivities (lower) in the Indian Ocean. The line indicates the transfer or diffusivity at the bottom of the layer.

ing coefficient be positive. The average diffusivity at interfaces below layer 5 and above the last interface is of $8.6 \pm 4 \mathrm{~cm}^{2} \mathrm{~s}^{-1}$ in the North Indian box. This value is much larger than the $1 \mathrm{~cm}^{2} \mathrm{~s}^{-1}$ global mixing required by a global 1-dimensional balance (Munk and Wunsch, 1998). The North Indian Ocean topography is relatively rough, which can lead to enhanced mixing, as suggested by microstructure measurements (e.g., Polzin et al., 1997). (In the Mozambique Channel, dianeutral exchanges are not significant owing to the large seasonal baroclinic variability there.) 


\section{Standard circulation summary}

The standard circulation is thus associated with an Agulhas Current of $74 \pm 7 \mathrm{~Sv}$ and with a relatively strong PIT of $15 \pm 5 \mathrm{~Sv}$. The overturning rate that I obtain is similar to the RT97 estimate. It is significantly larger than the Fu(1986) estimate (3.6 Sv) and smaller than the estimate of Macdonald (1998, $17 \pm 5 \mathrm{~Sv})$. On the other hand, a much weaker inflow is found consistently in GCM simulations (Zhang and Marotzke, 1999). But GCMs also have their limitations (weak vertical mixing, dependence upon climatological forcing, and low resolution of the deep boundary currents), and so their systematic error could easily explain my more vigorous result. Nevertheless, to test the hypothesis of small deep inflow, I tried to constrain the flow below $2000 \mathrm{dbars}$ to $0 \pm 2 \mathrm{~Sv}$. The model produced a $2 \pm 2 \mathrm{~Sv}$ northward flow below 2000 dbars by returning part of its $4 \pm 3$ Sv northward bottom inflow between 2500 and 3500 dbars, but the shallow meridional overturning of Lee and Marotzke $(1997,1998)$ was not found. There was no major violation in the conservation equations in the Indian Ocean. However the diancutral transfers were found significantly different from zero and oriented downwards in the subtropical and tropical regions, associated with negative diffusivities. Consequently, this solution was not retained.

In the standard solution, dianeutral advective and diffusive transfers are consistently determined and have magnitudes in the higher end of the previously published range.

\subsubsection{The Mozambique channel}

Obtaining a time-average circulation in the Mozambique channel is a challenge because the $\mathrm{I} 4$ and $\mathrm{I} 2 \mathrm{~W}$ sections were taken at different seasons and the $\mathrm{I} 2 \mathrm{~W}$ section is close to the Equator $\left(4^{\circ} \mathrm{S}\right)$, enhancing the noise in the geostrophic balance. Initial inversions produced a large and significant convergence in the intermediate and surface layers of the Mozambique Channel box. This convergence was found to be insensitive to the reference surface: the model adjusted the reference velocities to produce the same integrated flow for an initial sill-depth reference (2500 dbars) and an initial bottom reference. Because the convergence was significant, the original model was rejected. The error budget that I adapted from the Atlantic is too optimistic for this seasonally variable region. One could increase the a priori noise to account for the large variability in this region. The 
alternative that I adopted was to allow a large ageostrophic adjustment in the shallowest layer of $\mathrm{I} 2 \mathrm{~W}$ to accommodate the variability. This adjustment formally appears as the "Ekman" transport adjustment, Table 3.2.

After adjustment, mass is balanced within $1 \mathrm{~Sv}$ in individual layers (not shown). Because of the high variability, the vertical structure of the flow in the first layers is probably unrealistic. Nevertheless, the $14 \pm 6 \mathrm{~Sv}$ net flux through the Channel (Fig.3-3d, e) is relatively robust and consistent, within error bars with the different experiments that have been done in this region. DiMarco et al. (1998) obtained a bit larger southward transport (18 to $21 \mathrm{~Sv}$ ) assuming a zero velocity at the sill depth and below. But the relative transport below 2500 dbars was not included in their calculation (S. F. DiMarco, personal communication, 1998). To produce no flow below sill depth on $12 \mathrm{~W}$, important reference velocity adjustments were required, resulting in the smaller transport that I report.

\subsubsection{The Pacific Indian Throughflow (PIT)}

The mass flux through the PIT $(15 \pm 3 \mathrm{~Sv})$ is on the high side of the commonly accepted values (e.g., Meyers et al., 1995; Godfrey, 1996), but consistent with the preferred Fieux et al. (1994) value from the same JADE data. The silica flux through the JADE section is larger by a factor of $2\left(400 \pm 200 \mathrm{kmol} \mathrm{Sis}^{-1}\right)$ than what was estimated by RT97 from climatological silica concentration in the PIT region and a throughflow of $7 \mathrm{~Sv}$. The section was constructed by adding a station from the 1992 JADE section to the 1989 JADE data to close the box at the northern end. Such an addition is artificial and may introduce a bias because of the large seasonal variability in the Java Current. Sensitivity experiments revealed however that the presence of the added station was essential to permit enough range of adjustment to the model (a large a priori uncertainty and reference velocity adjustment were allowed at the added station pair). In particular, removing this station produced large silica fluxes below the PIT sill depth and increased the net silica flux through the section.

The PIT (Fig. 3-3f) shows a weak, uncertain net mass flux of intermediate waters flowing eastward ( $3 \mathrm{~Sv}$, layers $3-4,300$ to $800 \mathrm{dbars}$ ) in those layers. There is an apparent contradiction with the tongue of silica emanating from the PIT in the Wyrtki (1970) atlas. 
Fieux et al. (1994) discuss this eastward intermediate flux from those same 1989 data and find no inconsistency with the local water properties, the net flux being the sum of large and opposite fluxes of different waters types. (But as already described above, property tongues do not necessarily imply net mass flux. Any zero-mean time-dependent mass flux between the Pacific and the Indian Oceans which leaves behind fluid particles on the return flow would generate such a tongue (see Wunsch, 1996, p.79)). In any case, the silica flux in these layers is indistinguishable from zero. This intermediate water flux is enhanced when one removes the 1992 JADE station near Bali, and forcing the net intermediate silica flux to be westward causes large eastward silica and mass fluxes below sill depth on the JADE section, an unrealistic circulation. It was thus decided to leave the intermediate silica flux unconstrained.

The local formal uncertainty of $\pm 3 \mathrm{~Sv}$ on the PIT is probably optimistic because of the large baroclinic variability there (e.g., Meyers et al., 1995). A more realistic PIT uncertainty is given by the net mass flux uncertainty at $32^{\circ} \mathrm{S}$, that is, $\pm 5 \mathrm{~Sv}$.

\subsubsection{Full Property Equations and PO constraints}

A number of experiments were performed with the full property equations (that is, salt, heat and PO not expressed in terms of property anomalies). Use of full property equations has several drawbacks: The system is of lower rank, and it is difficult to weight the heat conservation equation properly. An uncertainty of $\pm 0.1 \mathrm{PW}$ in the heat equation is plausible in the thermocline; it is an overestimate in the deep layers characterized by smaller temperature gradients. In the anomaly equation formulation, the uncertainty can be attributed more consistently (Section 1.2.4), but the solution is, as always, directly dependent upon the reliability of the estimated error structure of the equations.

The solution with the full property equations (not shown) indicates a similar deep water inflow at $32^{\circ} \mathrm{S}$ but with increased transport $15-18 \mathrm{~Sv}$ as one goes north. For the practical reason mentioned above, heat conservation was not required. To balance mass, the model creates downward dianeutral fluxes in the subtropical and tropical boxes, with a larger upwelling occurring in the North Indian box. Several experiments showed that with the full property equations, the model could not produce a consistent circulation without 
those downward dianeutral fluxes. Although the circulations were not statistically different from the standard solution, those solutions were discarded.

An experiment with anomaly equations (including heat) but no PO constraints indicated a similar circulation with downward fluxes in the subtropical and tropical boxes.

\subsubsection{Indian Ocean summary}

Figure 3-3 shows my best-estimate of the net meridional (a function of depth and latitude) overturning streamfunction of the Indian Ocean as determined from the WOCE hydrographic sections both locally and globally. This estimate is consistent with both dynamics and simple biogeochemical principles (silica and "PO" conservation). Deep upwelling is returned primarily in the $800-1500$ dbars depth range, rather than near-surface.

1. A meridional overturning strength (measured by the rate of deep inflow) of $11 \pm 4 \mathrm{~Sv}$ at all three latitudes $\left(32^{\circ} \mathrm{S}, 20^{\circ} \mathrm{S}\right.$ and $\left.8^{\circ} \mathrm{S}\right)$ is consistently obtained, compatible with the RT97 estimate at $32^{\circ} \mathrm{S}$. Nevertheless, the global circulation requires modifications in the zonal and vertical partition of the deep flow found by RT97. Results from general circulation models have consistently found a weak deep inflow (Zhang and Marotzke, 1999) which is barely compatible, within an uncertainty of two standard deviations, with my results.

2. My estimate of the average Pacific-Indonesian Throughflow during the WOCE period (early $90 \mathrm{~s}$ ) is $15 \pm 5 \mathrm{~Sv}$ westward. The result is consistent with the global circulation, but I believe largely dictated by the use of the 1989 JADE cruise. The southward flux in the Mozambique Channel is of the same magnitude, implying a weak or null net flow between Madagascar and Australia.

3. Upwelling and down-gradient dianeutral mixing are found in the northern part of the Indian Ocean below the surface layers, with insignificant dianeutral transfers south of about $8^{\circ} \mathrm{S}$. The deduced "equivalent-horizontal average" diffusivity ranges between 2 and $10 \mathrm{~cm}^{2} \mathrm{~s}^{-1}$ while the upwelling velocity ranges between 1 and $3 \times 10^{-5} \mathrm{~cm} \mathrm{~s}^{-1}$. 


\subsection{The Pacific Ocean}

\subsubsection{Introduction}

While the Indian Ocean discussion was relatively extensive, I choose to keep the Pacific Ocean circulation description brief, the main focus being on the meridional overturning circulation (MOC). A large literature describes the Pacific circulation (e.g., Talley and Roemmich, 1991), and other studies are based on the same sections as the ones used here in the North Pacific (Roemmich and McCallister, 1989, hereafter RMC89; Macdonald, 1998) and in the South Pacific (Tsimplis et al., 1998; Wijffels et al., 1999, Wunsch et al., 1983).

\section{Deep water inflow}

In the South Pacific, regional 'estimates of the deep water inflow are relatively consistent. Wunsch et al. (1983) estimated net northward deep water flow of about $12 \mathrm{~Sv}$ using the Scorpio sections (Stommel et al., 1973; Warren, 1973) at $28^{\circ} \mathrm{S}$ and $43^{\circ} \mathrm{S}$. At $17^{\circ} \mathrm{S}$, a similar northward bottom flow of 11 to $14 \mathrm{~Sv}$ below $\theta=1.1^{\circ} \mathrm{C}$ was found from current meter and hydrographic measurements (Roemmich et al.,1996; Banks et al., 1995). Recent measurements in the western Southwest Pacific basin at $32^{\circ} \mathrm{S}$ evidenced a net northward flow of $15 \mathrm{~Sv}$ across a current meter array between the Tonga-Kermadec Ridge and $169^{\circ} \mathrm{W}$ and below 3250dbars, with a $\pm 10 \mathrm{~Sv}$ variability (Whitworth et al., 1999). Tsimplis et al. (1998), using the same WOCE sections that I use in the South Pacific, obtained a similar inflow of about $12 \mathrm{~Sv}$ at both latitudes. However, the global model of Macdonald (1998) yielded a moderate bottom water inflow of $8 \pm 5 \mathrm{~Sv}$ at $43^{\circ} \mathrm{S}$ from the older Scorpio data.

At $10^{\circ} \mathrm{N}$, Wijffels et al. (1996) estimated a deep inflow of $8 \mathrm{~Sv}$. In the North Pacific north of $24^{\circ} \mathrm{N}$, RMC89 estimated the circulation using an inverse model combing zonal and meridional sections and requiring top-to-bottom mass conservation. They obtained a net transport of bottom water of $10 \mathrm{~Sv}$ to the north across $24^{\circ} \mathrm{N}$ and of only $0.7 \mathrm{~Sv}$ to the north across $47^{\circ} \mathrm{N}$. In this same region, the Macdonald (1998) model yielded a weaker bottom water flux of $5 \pm 3 \mathrm{~Sv}$ at $24^{\circ} \mathrm{N}$, although it could be consistently constrained to a 10-Sv flow. She found no significant flow at $47^{\circ} \mathrm{N}$. As will be seen, a significant northward 
bottom water flow at $24^{\circ} \mathrm{N}$ is incompatible with conservation of heat and salt in the North Pacific.

\subsubsection{Model constraints}

\section{Data and treatment}

Figure 3-5 shows the Pacific Ocean sector of the model which is made of the pre-WOCE sections P1 (Aug. 1985) and P3 (Apr.-May 1985) and the WOCE sections P21 (Apr.Jun 1994) and P6 (May-Jul. 1992). I also attempted to use P4 (10N, Feb.-May 1989) but it produced large downward mass transfers at intermediate levels, probably because of the baroclinic variability in the Mindanao Current (S. Wijffels, personal communication, 1998) which made the section incompatible with the rest of the circulation. It was thus decided not to use $\mathrm{P} 4$ in the present model.

The hydrographic data were provided by the PIs L. Talley (P1), D. Roemmich and J. Swift (P3), M. McCartney (P6, P21, P4), H. Bryden (P6, P21, P4), Brady (P4) and J. Toole (P4, P6) either directly or through the WHPO center. I chose not to use P2 (Bando, Fukasawa and Okuda; Nov.-Feb 1994) because of the large time offset from the surrounding sections P3 and P1.

A gap over a few stations in the phosphate data set in P6 was filled by using a local nitrate-phosphate Redfield relation. As described above, relative geostrophic velocity was computed using horizontal extrapolation in the bottom triangles. In trenches where no horizontal extrapolation was possible, a polynomial was fit to the temperature. Any unrealistic-appearing shear in the triangles was set to zero shear.

\section{Layers and constraints}

The Pacific boxes defined by these sections are respectively the South subtropical box (P6P21); the central Pacific box (P21-P3-JADE89); the North Pacific box (P3-P1) and the Aleutian box (north of P1). Conservation equations were written for deep-water layers in the Aleutian box because water properties suggest that there is no deep water formation in the North Pacific (e.g., Warren, 1983), the deepest winter convection affecting layers 


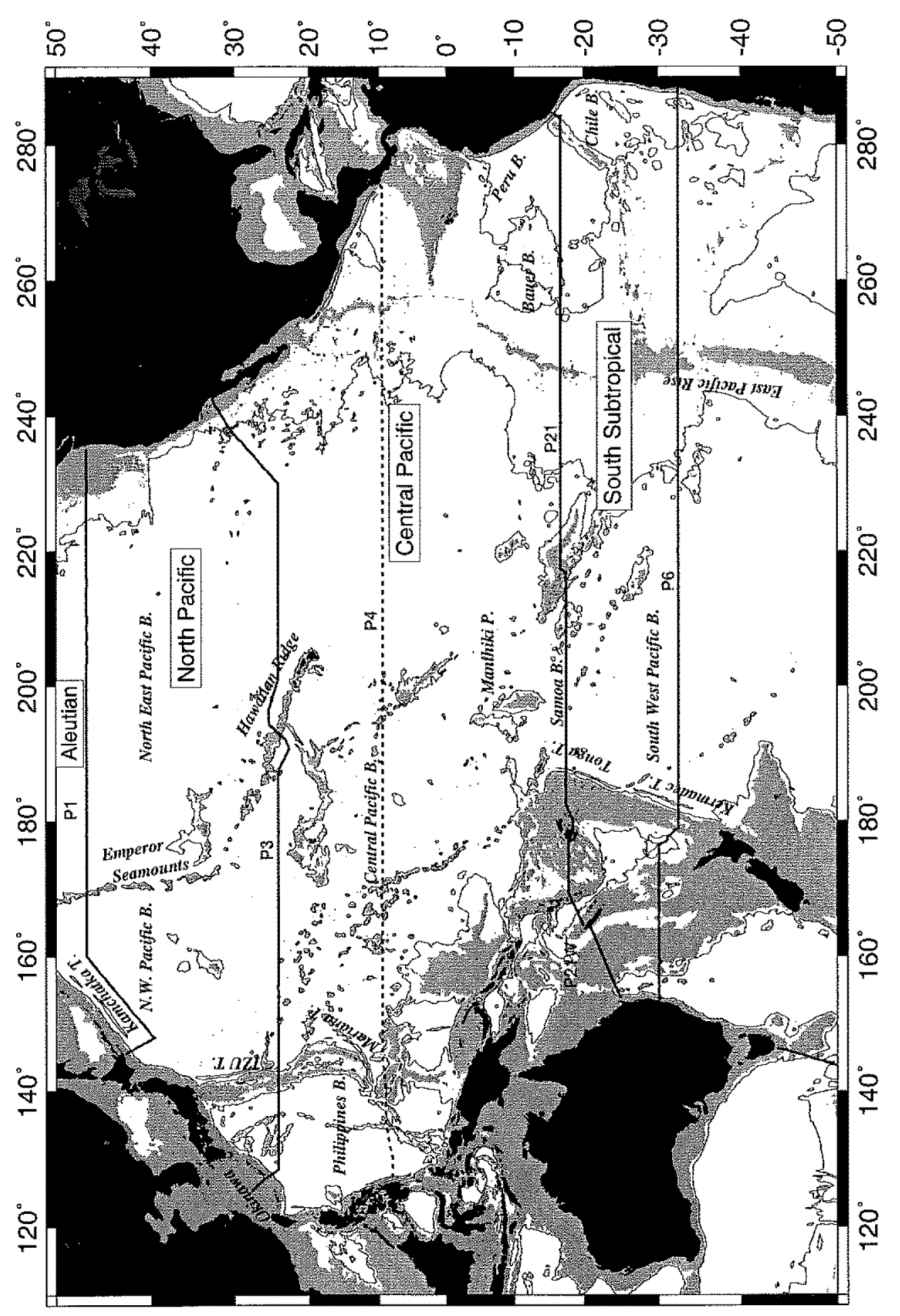

Figure 3-5: Pacific Ocean sections and topography. The $4000 \mathrm{~m}$ isobath is contoured and areas shallower than $3000 \mathrm{~m}$ are shaded. Box names are indicated in the shaded rectangles. 


\begin{tabular}{|c|c|c|c|}
\hline Layer & $\begin{array}{l}\text { Upper } \\
\text { interface }\end{array}$ & $24^{\circ} \mathrm{N}$ Properties & $\begin{array}{c}\text { Water mass } \\
\text { name }\end{array}$ \\
\hline 1 & surface & $\sigma_{\theta}=24.0 ; \theta=22.7$ & \multirow{2}{*}{ Surface Water } \\
\hline 2 & $\gamma^{n}=24.3$ & $\sigma_{\theta}=24.3 ; \theta=21.9$ & \\
\hline 3 & $\gamma^{n}=26$ & $\sigma_{\theta}=26.0 ; \theta=12.6$ & \multirow{5}{*}{ North Pac. Int. Water } \\
\hline 4 & $\gamma^{n}=26.5$ & $\sigma_{\theta}=26.4 ; \theta=9.1$ & \\
\hline 5 & $\gamma^{n}=26.8$ & $\sigma_{\theta}=26.7 ; \theta=7.1$ & \\
\hline 6 & $\gamma^{n}=27$ & $\sigma_{\theta}=26.9 ; \theta=5.9$ & \\
\hline 7 & $\gamma^{n}=27.15$ & $\sigma_{\theta}=27.0 ; \theta=5.2$ & \\
\hline 8 & $\gamma^{n}=27.35$ & $\sigma_{\theta}=27.2 ; \theta=4.4$ & \multirow{4}{*}{ Antarctic Int. Water } \\
\hline 9 & $\gamma^{n}=27.6$ & $\sigma_{2}=36.56 ; \theta=3.5$ & \\
\hline 10 & $\gamma^{n}=27.8$ & $\sigma_{2}=36.77 ; \theta=2.5$ & \\
\hline 11 & $\gamma^{n}=27.9$ & $\sigma_{2}=36.88 ; \theta=2.0$ & \\
\hline 12 & $\gamma^{n}=27.95$ & $\sigma_{2}=36.92 ; \theta=1.75$ & \multirow{5}{*}{ North Pac. Deep Water } \\
\hline 13 & $\gamma^{n}=28$ & $\sigma_{2}=36.97 ; \theta=1.5$ & \\
\hline 14 & $\gamma^{n}=28.05$ & $\sigma_{2}=37.01 ; \theta=1.3$ & \\
\hline 15 & $\gamma^{n}=28.09$ & $\theta=1.15$ & \\
\hline 16 & $\gamma^{n}=28.1$ & $\theta=1.10$ & \\
\hline 17 & $\gamma^{n}=28.11$ & $\sigma_{4}=45.885 ; \theta=1.06$ & \multirow{4}{*}{ Antarctic Bot. Water } \\
\hline 18 & $\gamma^{n}=28.125$ & $\sigma_{4}=45.900 ; \theta=1.02$ & \\
\hline 19 & $\gamma^{n}=28.15$ & $\theta=0.85$ & \\
\hline 20 & $\gamma^{n}=28.18$ & $\theta=0.85$ & \\
\hline
\end{tabular}

Table 3.5: Pacific Ocean neutral surface layers $\Gamma$ (in $\mathrm{kg} \mathrm{m}^{-3}$ ). Interfaces 15 and 17 have been used in the North Pacific only. The Macdonald (1998) water mass definition are indicated (from her table 17).

above 1000 dbars at $47^{\circ} \mathrm{N}$ (e.g. Reid, 1965, pp. 50-51; Reid, 1973; Talley et al., 1998, Fig. 10). Heat and salt are thus conserved in layers north of $47^{\circ} \mathrm{N}$ below $1000 \mathrm{dbars}$, allowing for dianeutral advective and diffusive fluxes. (With no constraints north of $47^{\circ} \mathrm{N}$, the model had an unphysical albeit non-significant southward net flow of $-2 \pm 2 \mathrm{~Sv}$ below 3000 dbars. Inclusion of the Aleutian box reduced this flow.)

The layers were suggested by John Toole (personal communication, 1998), optimized to match the Macdonald (1998) layers and to keep similar thicknesses over the whole Pacific (Table 3.5). The initial Ekman transports from the NCEP re-analysis are given in the second column of Table 3.6 .

As in other oceans, the circulation is required to conserve mass, salt, silica (top-tobottom); and "PO" and heat away from the surface. The net northward flux across P6 and P21 was constrained to the Bering strait $(0.8 \mathrm{~Sv}$, Coachman and Aagaard, 1988) +PIT 


\begin{tabular}{|c|r|r|c|}
\hline Section & Initial & \multicolumn{1}{|c|}{ Final } & \% correction \\
\hline P6 & $1.4 \pm 1$ & $1.3 \pm 1$ & $-10 \%$ \\
\hline P21 & $-18 \pm 8$ & $-20 \pm 6$ & $15 \%$ \\
\hline P3 & $7.5 \pm 3$ & $9 \pm 3$ & $13 \%$ \\
\hline P1 & $-4 \pm 2$ & $-3.9 \pm 1.6$ & $-3 \%$ \\
\hline
\end{tabular}

Table 3.6: Pacific initial Ekman transport and correction

flux ( $7 \mathrm{~Sv}$, see the discussion in the Indian Ocean section), resulting in a net constraint of $7.8 \pm 10 \mathrm{~Sv}\left[10=\sqrt{7^{2}+7^{2}}\right.$, where the first ' 7 ' is the a priori uncertainty in the throughflow and the second ' 7 ' is the variability in the baroclinic field according to the error budget]. The total flux across $\mathrm{P} 1$ and $\mathrm{P} 3$ was constrained to $0.8 \pm 3 \mathrm{~Sv}$ and $0.8 \pm 6 \mathrm{~Sv}$ respectively.

The deep water flow across $\mathrm{P} 6$ between $179^{\circ} \mathrm{W}$ and $168^{\circ} \mathrm{W}$ was constrained with the current meter measurements of Whitworth et al. (1999) with $16 \pm 5 \mathrm{~Sv}$ northward below $\gamma^{n}=27.95 ; \theta=1.75^{\circ}$. (The uncertainty of $\pm 5 \mathrm{~Sv}$ corresponds to their estimated variability in the baroclinic transport from repeat sections, which is half the total variability as measured from the current meters. Importantly, the authors found the P6 baroclinic transport to be representative of the average of 3 repeat sections.) Additional northward constraints were applied for each depth range, from the Whitworth et al. (1999) measurements and following Wijffels et al. (1999): 2000-3250 dbars: $1 \pm 3 \mathrm{~Sv} ; 3250-4250: 9 \pm 3 \mathrm{~Sv}$; 4250-bottom: $6 \pm 2.5 \mathrm{~Sv}$. (Because of my layer definition, the depth range separation is taken at 4250 dbars instead of the original 4750 dbars for which Whitworth et al. (1999) provided the transports (their Fig. 17). The uncertainties are assumed to take into account possible shifts in the transport.) Uncertainties correspond to half the total variability mentioned by Whitworth et al. (1999). The deep flow through P21 was similarly constrained with $12 \pm 4 \mathrm{~Sv}$ below $\gamma^{n}=27.95$, according to the analysis of Roemmich et al. (1996) in the Samoa Passage region. (Note that while Whitworth et al. (1999) estimated only the transport on the Western part of the deep water, Roemmich et al. (1996) estimated the total net northward transport of water colder than $\theta=1.1^{\circ} \mathrm{C}$.)

The deep flow was also constrained according to observed bathymetric features (Table 3.7). A relatively large tolerance of $\pm 5 \mathrm{~Sv}$ was given to the South West Basin, Yupanqui Basin $\left(20^{\circ} \mathrm{S}-105^{\circ} \mathrm{W}\right)$, and Peru-Chile Basin to allow dianeutral exchanges with layers above. 


\begin{tabular}{|l|l|l|l|l|}
\hline Name & Section & Layer depth & Net flux & Longitude \\
\hline \hline N. Tasmanian & P21W & $\gamma^{n}=28.1(3900)$ & $0 \pm 2 \mathrm{~Sv}$ & $153^{\circ} \mathrm{E}-156^{\circ} \mathrm{E}$ \\
\hline S. NewCal Trough & P6 & $\gamma^{n}=27.9(2000)$ & $0 \pm 2 \mathrm{~Sv}$ & $162^{\circ} \mathrm{E}-168^{\circ} \mathrm{E}$ \\
\hline Yupanqui Basin & P21 & $\gamma^{n}=28.05(3700)$ & $0 \pm 5 \mathrm{~Sv}$ & $94^{\circ} \mathrm{W}-78^{\circ} \mathrm{W}$ \\
\hline Bauer Basin & P21 & $\gamma^{n}=28.05(3900)$ & $0 \pm 2 \mathrm{~Sv}$ & $107^{\circ} \mathrm{W}-100^{\circ} \mathrm{W}$ \\
\hline N. Peru Basin & P21 & $\gamma^{n}=28.05(3900)$ & $0 \pm 2 \mathrm{~Sv}$ & $94^{\circ} \mathrm{W}-75^{\circ} \mathrm{W}$ \\
\hline Okinawa Trough & P3 & $\gamma^{n}=27.6(1200)$ & $0 \pm 2 \mathrm{~Sv}$ & $125^{\circ} \mathrm{E}-127^{\circ} \mathrm{E}$ \\
\hline N. Philippines Bas. & P3 & $\gamma^{n}=28.05(3100)$ & $0 \pm 2 \mathrm{~Sv}$ & $127^{\circ} \mathrm{E}-141^{\circ} \mathrm{E}$ \\
\hline Kuril Trench & P1 & $\gamma^{n}=28.11(4900)$ & $0 \pm 2 \mathrm{~Sv}$ & $145^{\circ} \mathrm{E}-147^{\circ} \mathrm{E}$ \\
\hline Name & Box & Layer depth & Net Div. & Longitudes \\
\hline \hline Tasmanian & P6/P21W & $\gamma^{n}=28.1(3900)$ & $0 \pm 2 \mathrm{~Sv}$ & $153^{\circ} \mathrm{E}-157^{\circ} \mathrm{E} / 153^{\circ} \mathrm{E}-156^{\circ} \mathrm{E}$ \\
\hline NewCal Trough & P6/P21W & $\gamma^{n}=27.9(2000)$ & $0 \pm 2 \mathrm{~Sv}$ & $162^{\circ} \mathrm{E}-168^{\circ} \mathrm{E} / 162^{\circ} \mathrm{E}-164^{\circ} \mathrm{E}$ \\
\hline S. Fiji Basin & P6 / P21 & $\gamma^{n}=28.0(2700)$ & $0 \pm 2 \mathrm{~Sv}$ & $173^{\circ} \mathrm{E}-179^{\circ} \mathrm{E} / 165^{\circ} \mathrm{E}-168^{\circ} \mathrm{E}$ \\
\hline South West Basin & P6 / P21 & $\gamma^{n}=28.05(3600)$ & $0 \pm 5 \mathrm{~Sv}$ & $179^{\circ} \mathrm{W}-122^{\circ} \mathrm{W} / 173^{\circ} \mathrm{W}-114^{\circ} \mathrm{W}$ \\
\hline Peru-Chile Basin & P6/P21 & $\gamma^{n}=28.05(3900)$ & $0 \pm 5 \mathrm{~Sv}$ & $112^{\circ} \mathrm{W}-71^{\circ} \mathrm{W} / 77^{\circ} \mathrm{W}-75^{\circ} \mathrm{W}$ \\
\hline
\end{tabular}

Table 3.7: Constraints imposed on the deep layer flux by bathymetric features. The constraints are imposed on either the net flux through a single section for a longitude range (upper part of the table, positive northward) or on the net divergence (Div.) in a box between two sections (lower part of the table). The layer and its approximate depth below which the flux is constrained are indicated, along with the uncertainty on the net flux (section) or divergence (box) (in Sv).

\section{Reference surfaces and dianeutral terms}

The reference surfaces, indicated in Table 3.8, were chosen roughly at the top of the northward-flowing Circumpolar Deep Water. At $32^{\circ} \mathrm{S}$, near the deep western boundary current against the slope of the Kermadec ridge, the current meter results of Whitworth et al. (1999) showed that the limit of the northward-flowing waters did not coincide with any "water-mass boundary" as classically used as reference surfaces. The initial velocity field was thus adjusted to match their 2-year mean absolute velocity. At $17^{\circ} \mathrm{S}$ against the Tonga Ridge, the reference surface was moved just above the high shear region of the deep western boundary current. Away from the boundary currents, I chose $\theta=1.1^{\circ}$ or $1.3^{\circ}$ as a reference (i.e., Banks et al., 1995; Tsimplis et al., 1998). However, an experiment with reference surface on $\gamma^{n}=27.95\left(\theta \simeq 2^{\circ}\right)$ showed that integrated mass transports after inversion are relatively insensitive to reference surface choice in this area. Along sections P3 and P1, the reference surfaces were chosen based on RMC89. Dianeutral terms and reference velocity adjustment were chosen as for the Indian Ocean, page 80 . 


\begin{tabular}{|l|l|l|}
\hline Section & Reference surface & Remark \\
\hline P6 & $\gamma^{n}=28.10 ; \theta \simeq 1.1^{\circ}$ & match to Whitworth et al. (1999) in DWBC \\
\hline P21 & $\gamma^{n}=28.10 ; \theta \simeq 1.3^{\circ}$ & Banks et al. (1995) \\
\hline P3 & $\gamma^{n}=27.95 ; \theta \simeq 1.6^{\circ}$ & 2000 dbars Roemmich and McCallister (1989) \\
\hline P1 & $\gamma^{n}=28.09 ; \theta \simeq 1.1^{\circ}$ & 4000 dbars initial velocity similar to RMC89 \\
\hline
\end{tabular}

Table 3.8: Reference surfaces in the Pacific Ocean.

a) South Subtropical $32 \mathrm{~S}-17 \mathrm{~S}$

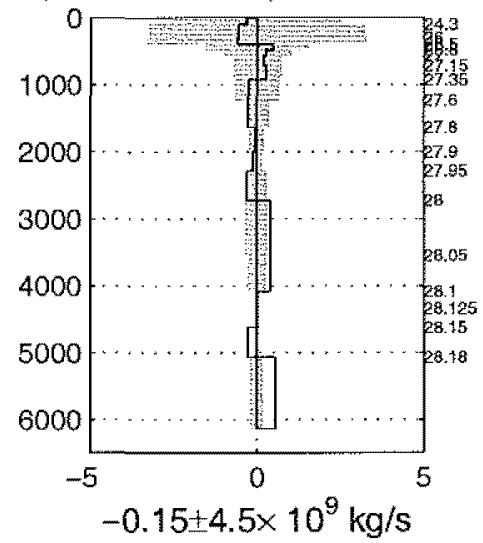

b) Central Pacific 17S-24N

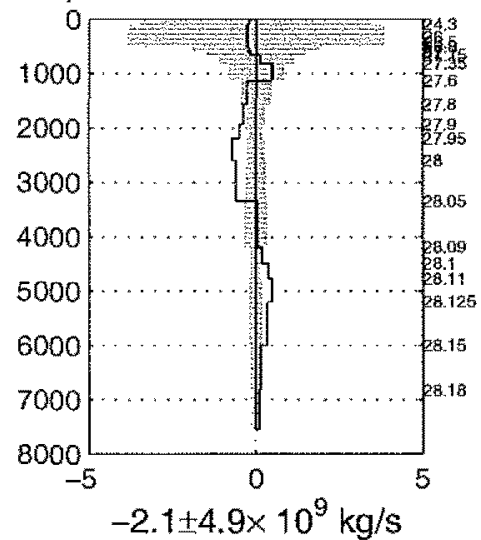

c) North Pacific $(24 \mathrm{~N}-47 \mathrm{~N})$

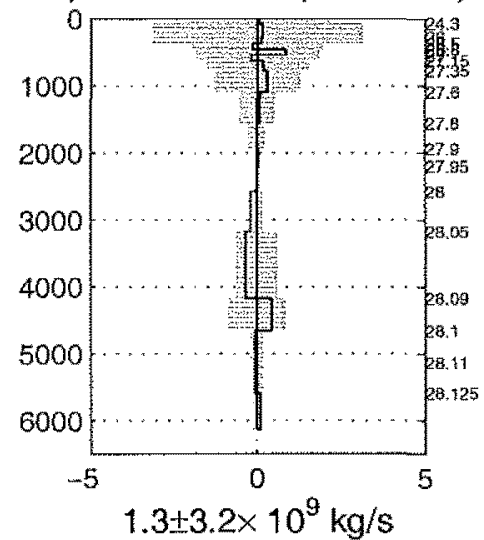

Figure 3-6: Standard solution mass residuals in a) the South Pacific box, $30^{\circ} \mathrm{S}-17^{\circ} \mathrm{S}$; b) the Central Pacific box, $17^{\circ} \mathrm{S}-24^{\circ} \mathrm{N}$ and c) the North Pacific box, $24^{\circ} \mathrm{N}-47^{\circ} \mathrm{N}$. The line gives the residual in invidual layers (in $10^{9} \mathrm{~kg} \mathrm{~s}^{-1}$ ) and the shaded area give the one standard deviation uncertainty. The top-to-bottom residual is indicated below the graph.

\subsubsection{Pacific Ocean circulation}

Almost all residuals of the equations of the "standard" solution were within the one standard deviation a priori range. The Whitworth et al. (1999) constraint of net flow of $16 \pm 5 \mathrm{~Sv}$ across their current meter array was met with $11 \pm 3 \mathrm{~Sv}$; however, constraints on the vertical partition of the flow were not met within one standard deviation, but within two standard deviations of the a priori: the model produced northward transport higher than the ones prescribed below $4250 \mathrm{dbars}, 10 \pm 2 \mathrm{~Sv}$, and lower just above, $4 \pm 1 \mathrm{~Sv}$.) Mass residuals in individual layers are smaller than $0.5 \mathrm{~Sv}$ and all indistinguishable from zero within two standard deviations (Fig, 3-6). Small adjustments were made to the initial Ekman transport ( 3 to $15 \%$, Table 3.6 ).

Again, the discussion that follows is focused on the large scale integrated fluxes. However, reference velocities, absolute velocities and water-mass transports accumulated from 
a) P6 (32S)

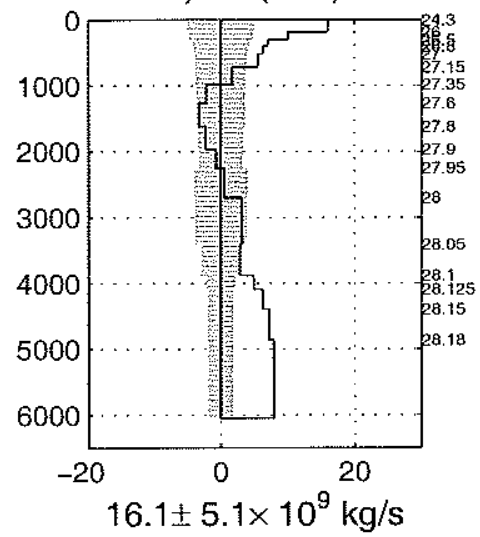

d) P3 (24N)

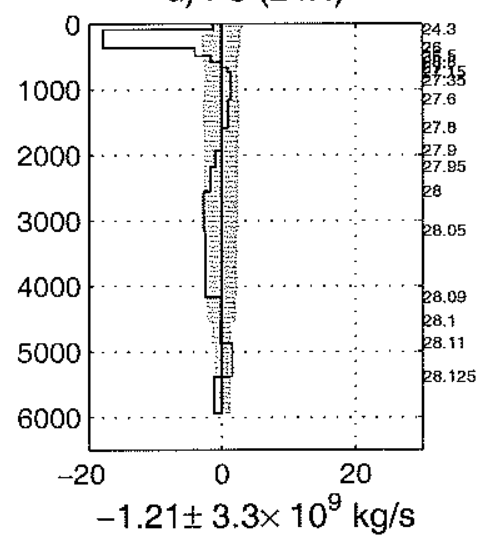

b) $\mathrm{P} 21 \mathrm{~W}+\mathrm{P} 21(17 \mathrm{~S})$

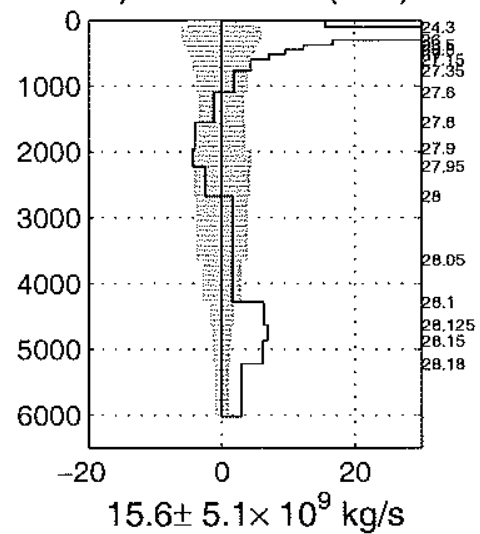

e) $\mathrm{P} 1(47 \mathrm{~N})$

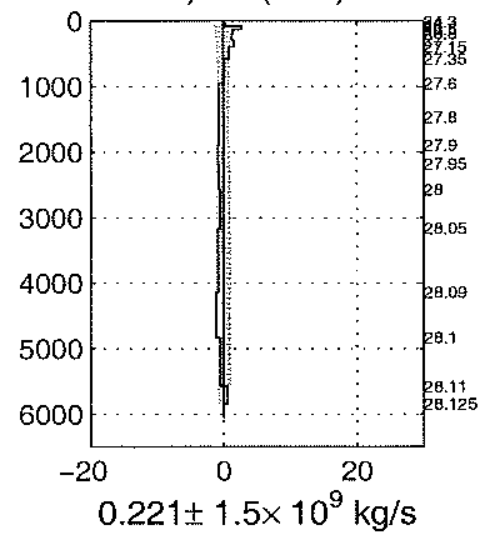

c) P21W only

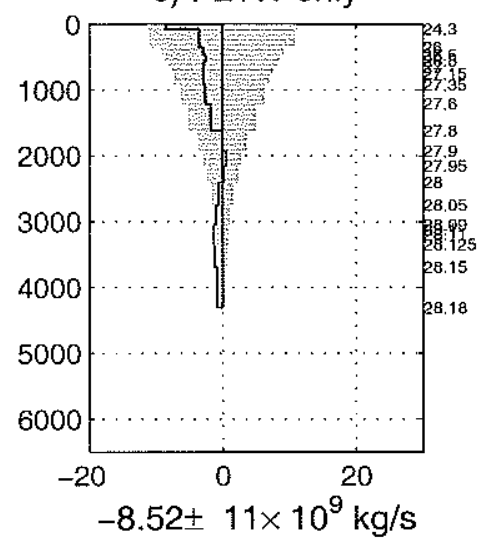

Figure 3-7: Pacific Ocean standard circulation. The mass transport across each section is shown, integrated from the bottom (the overturning streamfunction). The shaded area corresponds to the uncertainty (one standard deviation). The net transport is indicated below each graph. While panel b) gives the total transport across $17^{\circ} \mathrm{S}$, panel c) gives the transport between New Caledonia and Australia only (P21W).

west to east are given in Appendix D (pages 210-219) for reference. The integrated flow for the standard solution for the Pacific Ocean circulation is shown on Figure 3-7. (Again, overturning streamfunctions are used, the slope of the curve indicating the direction of the flow).

\section{Net fluxes}

The net fluxes across $32^{\circ} \mathrm{S}$ and $17^{\circ} \mathrm{S}$ corresponding to the Pacific-Indonesian Throughflow (PIT) plus Bering Strait transport are estimated to be $16 \pm 5 \mathrm{~Sv}$ going north. The total 
flow between New Caledonia and Australia is uncertain and to the south, $-8 \pm 11 \mathrm{~Sv}$ (Fig. 37c), implying a $24 \pm 12 \mathrm{~Sv}$ northward flux between New Caledonia and Chile. Tsimplis et al. (1998) obtained similar transports. At $32^{\circ} \mathrm{S}$ in the Tasman Sea, however, the total transport between Australia and $170^{\circ} \mathrm{E}$ is also southward, with about $-10 \mathrm{~Sv}$, in apparent contradiction with Wijffels et al. (1999) who found a closed circulation in the Tasman Sea implying no net transport.

\section{Deep inflow}

In the South Pacific, net northward flow of deep water is found at approximately $8 \mathrm{~Sv}$ (Fig. 3-7a, b). At $32^{\circ} \mathrm{S}$, most of the flow occurs in the bottom layer, below $\gamma^{n}=28.18$ $\left(\theta \simeq 0.8^{\circ}\right)$ at $8 \pm 2 \mathrm{~Sv}$, while at $17^{\circ} \mathrm{S}$ the bulk of the northward flow occurs higher in the water column, with $7 \pm 1.3 \mathrm{~Sv}$ below $\gamma^{n}=28.1\left(\theta \simeq 1.2^{\circ}\right)$. Dianeutral transfers (described below) account for this shift. The deep western boundary current against the TongaKermadec ridge at $32^{\circ} \mathrm{S}$ has a strength of $17 \pm 3 \mathrm{~Sv}$ below $\gamma^{n}=27.95\left(\theta \simeq 1.9^{\circ}\right)$ (page 211). (The DWBC was actually flowing south at the time of section P6, but probably as a result of a strong barotropic fluctuation, thus not visible in the hydrographic data.) The total flow between the Tonga-Kermadec Trench and $168^{\circ} \mathrm{W}$ is weaker but still consistent within error bars with the Whitworth et al. (1998) current meter results with $11 \pm 3 \mathrm{~Sv}$ between $179^{\circ} \mathrm{W}$ and $168^{\circ} \mathrm{W}$ below $\gamma^{n}=27.95\left(\theta \simeq 1.9^{\circ}\right)$.

In the North Pacific at $24^{\circ} \mathrm{N}$ (Fig. 3-7d) the deep inflow is indistinguishable from zero in the bottom layers below $\gamma^{n}=28.09\left(\theta \simeq 1.15^{\circ}\right)$, thus much weaker than the RMC89 flow of $10 \mathrm{~Sv}$. Still, there is a total eddy ${ }^{1}$ transport of northward-flowing water of $7 \pm 6 \mathrm{~Sv}$ below $\theta=1.15^{\circ}$, but this same amount returns south in the same isopycnal depth range.

The deep inflow into the Pacific Ocean estimated with this model is thus lower than most of the published estimates mentioned above that found 10 to $13 \mathrm{~Sv}$ from regional analysis (e.g., Wijffels et al., 1999; Whitworth et al. 1999; Tsimplis et al., 1998; Wunsch et al., 1983) and 5 to $8 \mathrm{~Sv}$ from the global Macdonald model. The RMC89 circulation conserved mass only for the top-to-bottom integrated transport within boxes enclosed

\footnotetext{
${ }^{1}$ Northward eddy transport is defined here as the sum, over all station pairs, of the flow that is oriented northward within the considered depth range.
} 
by zonal and meridional sections: no conservation statements were applied in individual layers. (They used the same $24^{\circ} \mathrm{N}$ and $47^{\circ} \mathrm{N}$ sections as I do but added a zonal section at $36^{\circ} \mathrm{N}$ and several meridional sections.) Their estimated $10 \mathrm{~Sv}$ inflow implies a large dianeutral velocity of $3 \times 10^{-4} \mathrm{~cm} \mathrm{~s}^{-1}$ (RMC89, page 201) and an associated diapycnal diffusivity of around $60 \mathrm{~cm}^{2} \mathrm{~s}^{-1}$. Such a diffusivity is in the upper range of local measurements in the presence of rough topography (e.g., Polzin et al., 1997) whereas the North Pacific topography is relatively smooth.

The net deep inflow in the standard solution is limited mainly by the conservation equations in the North Pacific deep layers, and in particular by the salinity anomaly conservation equations. An experiment with heat conservation only, produced a $5 \mathrm{~Sv}$ deep inflow with strong upwelling $\left(w^{*} \simeq 4 \times 10^{-5} \mathrm{cms}^{-1}\right)$ and vertical mixing $\left(60 \mathrm{~cm}^{2} \mathrm{~s}^{-1}\right)$ at $4000 \mathrm{dbars}$ between $24^{\circ} \mathrm{N}$ and $47^{\circ} \mathrm{N}$. An experiment with a deep reference surface $\left(\gamma^{n}=\right.$ 28.11, which is close to the upper limit of the deep inflow found by RMC89) produced a qualitatively similar circulation. It was tried to constrain the deep infow to $10 \pm 5 \mathrm{~Sv}$ to the north, but the model could not meet the constraint and the circulation and property fluxes remained almost unchanged. The RMC89 circulation in the North Pacific could be reproduced only by suppressing conservation in individual layers. The Macdonald (1998) circulation, which produced a more moderate inflow of $5 \pm 3 \mathrm{~Sv}$ at, $24^{\circ} \mathrm{N}$, had no heat conservation constraints, and the fact that anomaly equations were not used gave the salt conservation equation a much lower weight in her result. Salt and heat conservation thus greatly limit the net inflow of bottom water into the North Pacific basin north of $24^{\circ} \mathrm{N}$, and in turn also limit the inflow in the South Pacific. As with the case of the Indian Ocean meridional overturning, it is not known whether this low inflow is an actual feature or whether it is model dependent, for instance due to poor representation of vertical exchanges.

Because no net deep flow enter the North Pacific, the deep inflow in the South Pacific Ocean is all upwelled between $17^{\circ} \mathrm{S}$ and $24^{\circ} \mathrm{N}$. The estimate of the South Pacific deep inflow is related with the weight of the PO constraint. An experiment with no PO constraints yielded a $14.8 \pm 3 \mathrm{~Sv}$ deep inflow associated with larger mass residuals at intermediate and deep levels. This solution is physically acceptable as well. However, the two solutions 
are in apparent contradiction (the uncertainties on the deep inflow of the two solutions hardly overlap within two standard deviations at $10 \mathrm{~Sv}$ ). This contradiction suggests that the a priori weights on some of the constraints may be too high. In particular, there is a trade-off between the constraints on the deep inflow derived from the current meter data of Whitworth et al. (1999), the PO constraint, and mass residuals in the Central Pacific. The current meter data may be hampered by the shortness of the time series (22 months), while the weight on the PO constraints was chosen analogously to the salinity, according to the "ad-hoc" method described on Section 1.2.4 (page 22). I chose the standard solution as the one of low-overturning because of the smaller mass residuals, but this choice is, to some extent, arbitrary.

\section{Deep, intermediate and surface flow}

The horizontal flow structure in the density class defining the different water masses defined in Table 3.5 are given in Appendix D (Figures D-16-D-24). The deep water flow in the South Pacific is directed southward as found by Wunsch et al. (1983), Macdonald (1998) and Tsimplis et al. (1998). Most of this flow occurs between $170^{\circ} \mathrm{W}$ and $140^{\circ} \mathrm{W}$ at $32^{\circ} \mathrm{S}$ (Fig.D-16). Such a deep flow contrasts with the speculative interior circulation that Whitworth et al. (1999) inferred based on a Stommel and Arons (1960) schematic and current meter measurements near the Kermadec Trench. But the present circulation is consistent with tracer conservation, including silica conservation over the Pacific Basin (Section 4.3.1).

In the South Pacific at $30^{\circ} \mathrm{S}$ (P6, Fig. D-16), the East Australian Current closes the subtropical gyre with a strength of $-36 \pm 10 \mathrm{~Sv}$ southward in the region between Australia and $154.5^{\circ} \mathrm{E}$ and from the surface to $\gamma^{n}=27.95$, similar to previous estimates (e.g., Macdonald, 1998, page 348; Chiswell et al., 1997). (The formal uncertainty of the boundary current transports are underestimated because the error budget did not take into account baroclinic variability on small scales.) At $25^{\circ} \mathrm{S}$, the estimated East Australia Current strength is somewhat smaller at $-25 \pm 6 \mathrm{~Sv}$ (from Australia to $155^{\circ} \mathrm{E}$ ). Both estimates are also consistent with those of Tsimplis et al. (1998). At both latitudes, an immediate recirculation returns about $80 \%$ of the flow to the north within $500 \mathrm{~km}$ of the current. The 
Peru-Chile Current is apparent on the eastern side of P6 (Fig. D-16), carrying 8.5 44 Sv to the north at $30^{\circ} \mathrm{S}$ (surface to $\gamma^{n}=27.95 ; 75^{\circ} \mathrm{W}$ to Peru). At $16^{\circ} \mathrm{S}$ (P21E), the flow is to the south against the coast $(-8 \pm 2 \mathrm{~Sv}$ ), with a $22 \pm 2 \mathrm{~Sv}$ northward jet offshore (Fig. D-20) and eddy-like motion farther west.

In the North Pacific (Figs. D-22-D-24), the intermediate and surface flow field is very similar to that of Macdonald (1998) at both sections, the differences arising mainly in the deep layers. The subtropical gyre at $24^{\circ} \mathrm{N}$ is closed by the Kuroshio (68 $17 \mathrm{~Sv}$ to the north between the surface and $\gamma^{n}=27.95$ and from the western boundary to $130^{\circ} \mathrm{E}$ ). A similar flow of $60 \mathrm{~Sv}$ was reported from earlier data (Worthington and Kawai, 1972, page 373). About $2 / 3$ of this flow is returned south between $132^{\circ} \mathrm{E}$ and $134^{\circ} \mathrm{E}$ and $1 / 3$ over the rest of the basin. The Kuroshio transport in the East China Sea alone, $28 \pm 6 \mathrm{~Sv}$, is consistent with estimates from Macdonald (1998) and RMC89. However, recent current meter observations from the WOCE PCM-1 array $400 \mathrm{~km}$ upstream suggest a somewhat smaller flux of $22 \pm 2.5 \mathrm{~Sv}$ across the strait between Taiwan and the Ryukyu Islands (Lee et al., 1999; Johns et al., 1999). It is not known whether the difference is due to interannual variability (e.g., Ichikawa and Beardsley, 1993), or to additional mass influx through the Ryukyu Islands. At $47^{\circ} \mathrm{N}$, a northward flow is found close to the coast ( $31 \pm 7 \mathrm{~Sv}$ from the surface and $\gamma^{n}=27.95$ and west of $146.5^{\circ} \mathrm{E}$ ), adjacent to the Oyashio that carries $-37 \pm 2 \mathrm{~Sv}\left(146.5^{\circ} \mathrm{E}\right.$ to $\left.148.4^{\circ} \mathrm{E}\right)$ south. On average, southward flow is expected against the coast just north of this region, but the $\mathrm{P} 1$ section reaches the coast close to the Kuroshio Front, which generates warm eddies (Kawai, 1972, page 248). The northward flow that is found against the coast is probably associated with such an eddy (i.e., Talley et al., 1991).

\section{Dianeutral transfers and diffusivity}

Dianeutral mass transfers indicate upwelling at the surface in the Central Pacific (Fig.3$8 \mathrm{~b}, 45 \pm 6 \mathrm{~Sv})$ and downwelling in the subtropical gyres (-16 $\pm 5 \mathrm{~Sv}$ in the South Pacific; $-17 \pm 3 \mathrm{~Sv}$ in the North Pacific)(Fig.3-8a,c). At depth, and in the South and Central Pacific, there is upwelling below 2000 dbars associated with systematic and significant diffusivities ranging from 1 to $8 \pm 2 \mathrm{~cm}^{2} \mathrm{~s}^{-1}$ between 2000 and 4500 dbars (Fig.3-8a,b). Diffusivities across the deepest layer interface are larger at about $40 \mathrm{~cm}^{2} \mathrm{~s}^{-1}$. The large diffusivities 
a) South Subtropical $32 S-17 \mathrm{~S}$
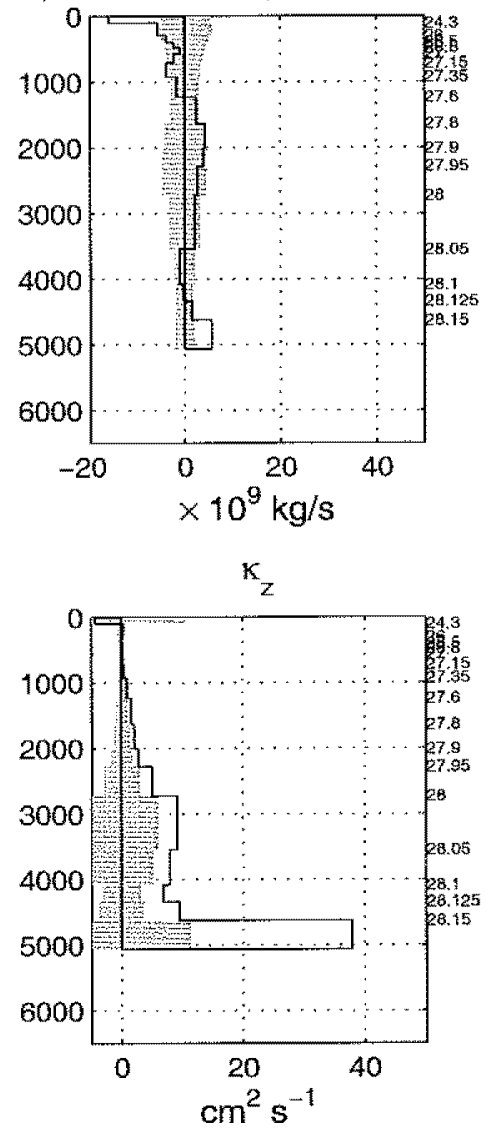

b) Central Pacific $17 \mathrm{~S}-24 \mathrm{~N}$
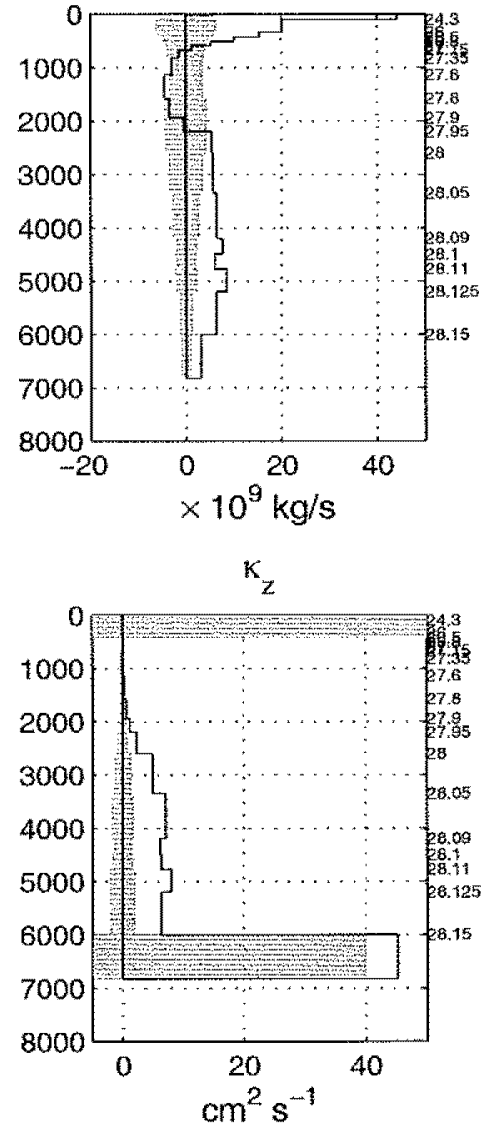

c) North Pacific $(24 \mathrm{~N}-47 \mathrm{~N})$
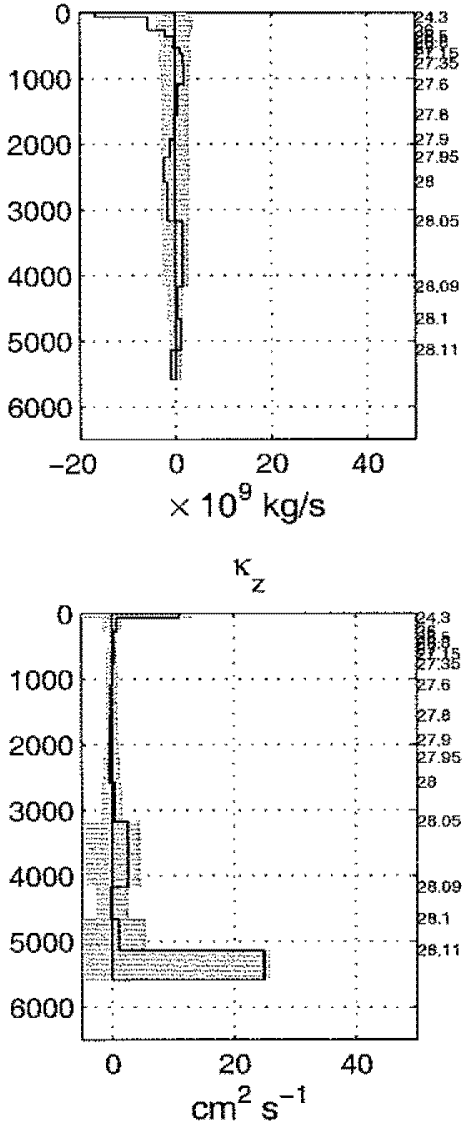

Figure 3-8: Dianeutral transfers (upper) and diffusivities (lower) in the Pacific Ocean. The line indicates the transfer or diffusivity at the bottom of the layer. 
are related to small horizontal interface areas. But the area calculation is uncertain for the deep layers as it was derived using Levitus (1994). A back-of-the-envelope heat balance by RMC89 and Roemmich et al.(1996) suggested possibly dianeutral diffusivities of 60 to $1000 \mathrm{~cm}^{2} \mathrm{~s}^{-1}$ in the deep layers downstream from the Samoan Passage. In the deep North Pacific, dianeutral diffusivities are positive, but not significant. North of $47^{\circ} \mathrm{N}$ (not shown), dianeutral transfers are not distinguishable from zero. Average values for dianeutral diffusivities and velocities are given at the end of this chapter.

The magnitudes of the vertical transfers and diffusivities are within the range of previously published values (e.g., Tsimplis et al., 1998; Macdonald, 1998; Wijffels, 1993). Tsimplis et al. (1998) obtained a smaller upwelling of a few tenths of a Sverdrup below 3500 dbars and downwelling above 3500 dbars. Macdonald (1998) obtained no significant vertical exchanges there. On the other hand, she estimated significant upwelling in the North Pacific which I do not because of the weaker deep inflow discussed above.

\subsubsection{Pacific circulation summary}

The standard estimate of the Pacific circulation is associated with an inflow of CDW that is weaker than most previous estimates. The small size of the inflow is related to conservation of heat and salt in the deep North Pacific layers, which precludes significant net inflow across $24^{\circ} \mathrm{N}$. As a result, the inflow from the South is limited also, and most of the circumpolar deep water (CDW) moving northward across $32^{\circ} \mathrm{N}$ upwells in the Central Pacific. By removing the PO conservation constraint, a solution with higher CDW inflow in the South Pacific was obtained, but the associated mass residuals increased in the Central Pacific. I chose, arbitrarily to some extent, to retain the PO-conserving circulation as my standard solution. The bulk of the bottom water inflow returns at deep levels so that the deep overturning cell is isolated, in the integrated sense, from the intermediate and surface water circulation. The South Pacific thermocline flow is directed northward, suggesting that the density class found in the Pacific-Indonesian Throughflow ultimately originates in the South Pacific Ocean south of $32^{\circ} \mathrm{S}$. (However, the pathway is complicated, e.g., Fine et al. (1994)). Such a circulation was also inferred by previous authors (e.g., Macdonald, 1998; Wijffels et al., 1996; RMC89; Wunsch et al., 1983). 
Dianeutral diffusivities in the deep layers indicate significant and consistent values in the range $2-8 \mathrm{~cm}^{2} \mathrm{~s}^{-1}$, with higher values in the bottom layers. The model transports of surface boundary currents are consistent with previous estimates. 


\subsection{The Atlantic Ocean}

\subsubsection{Introduction}

The North Atlantic Ocean is the best documented in comparison with the other oceans. The meridional overturning circulation consists of 13 to $30 \mathrm{~Sv}$ northward flow of thermocline water coming from the South Atlantic that eventually sinks at high latitudes to form the southward flowing North Atlantic Deep Water (NADW). The NADW flows to the south, mainly in the Deep Western Boundary Current (DWBC) whose transport increases owing to the contribution of Atlantic Antarctic Intermediate Water (AAIW) from above and Antarctic Bottom Water (AABW) from below. The net NADW export to the Southern Ocean is approximately $20 \mathrm{~Sv}$ according to most authors. AABW is formed in the Weddell Sea and around the continental shelf of Antarctica and flows north in the Atlantic at a rate of about $8 \mathrm{~Sv}$ at $30^{\circ} \mathrm{S}$, according to current meter measurements (Holfort et al., 1999b, $8 \mathrm{~Sv}$; Hogg et al., 1999, $7 \mathrm{~Sv}$ ). Part of this flow is entrained into southward flowing NADW within the Brazil Basin; about 2 Sv enters the Guiana Basin (Hall et al., 1997) and 1.2 Sv enters the Eastern Atlantic through the Romanche Fracture Zone (Mercier and Speer, 1998).

Elements of this large-scale circulation has been estimated quantitatively in several studies, including the inverse box model approaches of Wunsch (1978, western North Atlantic); Wunsch and Grant (1982, whole North Atlantic); Hall and Bryden $\left(1982,24^{\circ} \mathrm{N}\right)$; Roemmich and Wunsch (1985) and Rintoul and Wunsch (1991) (subtropical North Atlantic). In the South Atlantic, the circulation was estimated by Fu (1986); Lux (1997, equatorial region); Holfort et al. (1999); and Saunders and King (1995, 45 $\left.{ }^{\circ} \mathrm{S}\right)$. But the most comprehensive circulation picture probably comes from the global model of Macdonald (1998). Besides box section models, a variety of other inverse models has been used, e.g., Martel and Wunsch (1993, linear, finite-difference, steady); Mercier et al. (1993, non-linear) and Schlitzer (1988, 1993, coarse resolution, intermediate complexity model), each bearing its own skills and drawbacks.

In addition to those estimates, recent qualitative pictures of the North Atlantic circulation and its connection with other basins have emerged from water mass analysis and 
current meter measurements by Reid $(1994,1996)$ and a literature review by Schmitz and McCartney (1993) and Schmitz (1996a). (The two later reviews include quantitative estimates from many authors, but the resulting estimate of the circulation is presented with no error bars.)

The different water mass transports presented in these papers are discussed below in a quantitative comparison with my results.

\subsubsection{Model constraints}

\section{Data and treatment}

Figure 3-9 shows the Atlantic Ocean sector of the model which is made of the WOCE sections A2 (Gauss 226, Jul. 1993, Koltermann et al., 1999); A5 (Jul. 1992, Parilla et al., 1994); A6 (Feb. 1993) and A7 (Jan 1993) (Arhan et al., 1998; Oudot et al., 1998); A8 (Mar. 1994), A9 (Feb. 1991) and A10 (Dec. 1993) (Siedler et al., 1996); and A11 (Dec. 1993)(Saunders and King, 1995). I was not able to incorporate the available non-WOCE-standard data at $36^{\circ} \mathrm{N}$ (see page 14). At $26^{\circ} \mathrm{N}$ in the Florida Strait (a continuation of section A5), the 1992 data did not contain nutrients, so that CTD and nutrient data from the 1981 AT109 section of Roemmich and Wunsch (1985) were used. The low latitude and small station spacing enhanced the noise in geostrophic velocities across the A6 and A7 sections, in particular against the coast of Brazil. To decrease the noise, a horizontal Gaussian filter with cutoff at $70 \mathrm{~km}$ was applied to the temperature and salinities in the upper $1000 \mathrm{dbars}$. Because the A6 section does not include the part of the North Brazil Current that flows on the continental shelf, a 5-Sv transport was added to the section (see below).

The hydrographic data were provided by the PIs P. Koltermann (A2); G. Parilla and R. Millard (A5); M.Arhan, A. Morlière, C. Oudot, C.Colin and Y.Gouriou (A6, A7); G. Siedler, T. Mueller and R. Onken (A8, A9, A10) ; and P. Saunders (A11), either directly or through the WHPO Center when public. 


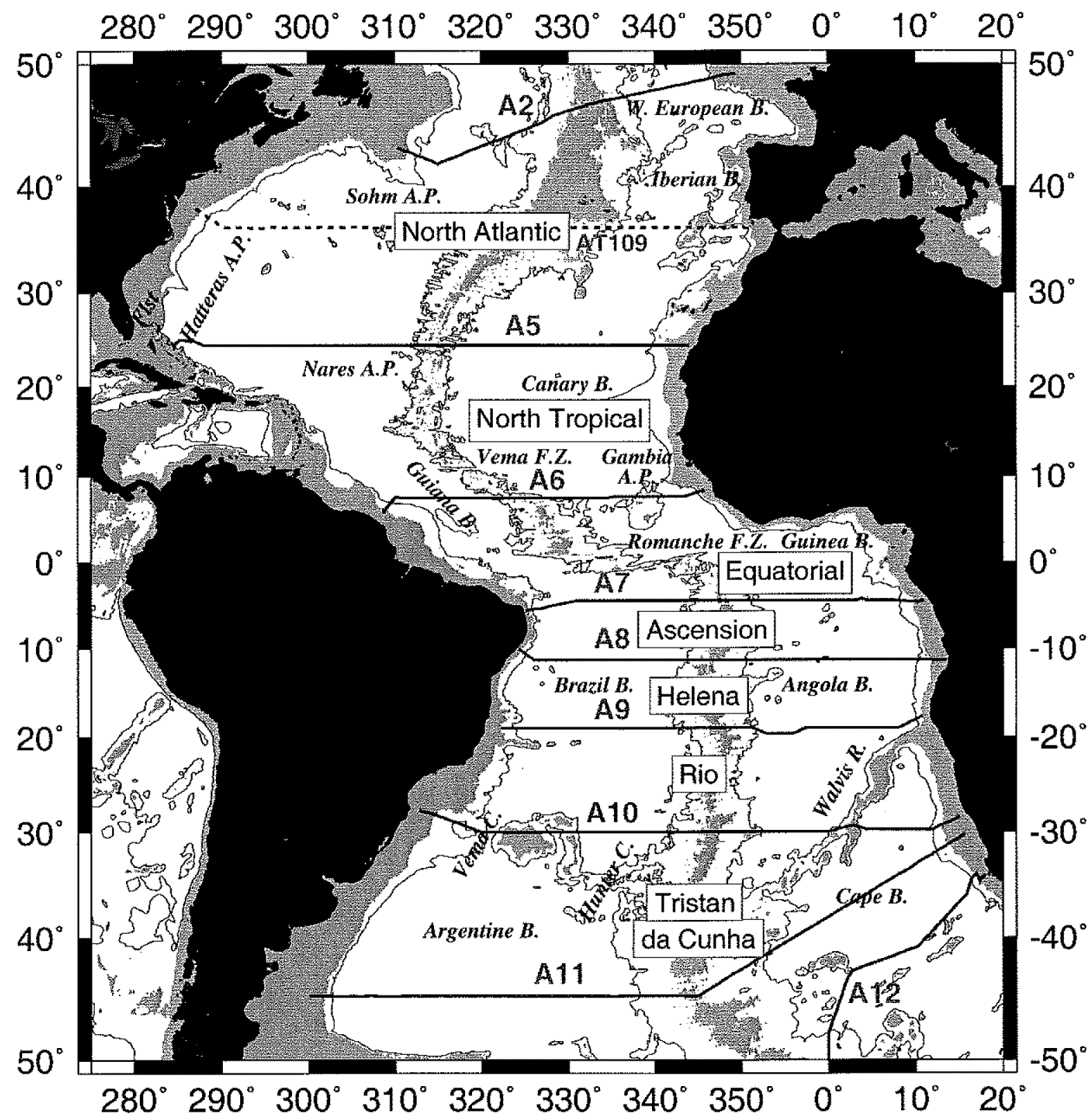

Figure 3-9: Atlantic Ocean sections and topography. The $4000 \mathrm{~m}$ isobath is contoured and areas shallower than $3000 \mathrm{~m}$ are shaded. The light rectangles indicate the box labels. 


\section{Layers and constraints}

The Atlantic boxes (Figure 3-9) are respectively the North Atlantic box (A2-Florida StraitA5); the North Tropical box (A5-A6); the Equatorial box (A6-A7); the South Tropical boxes "Ascension" (A7-A8) and "Helena" (A8-A9); the South subtropical "Rio" box (A9A10) and the South subtropical "Tristan da Cunha" box (A10-A11). In addition, a super "Central Atlantic" box was defined between A5 and A9 to impose tighter conservation constraints between those two sections. Without this super box, coupling between the flow north and south of the Equator is loose because of large noise in the equatorial region transport calculations.

In the North Atlantic, the neutral surface layers were chosen to match the Macdonald (1998) layers and water mass definition (Table 3.9) for comparison with her results and other authors (e.g., Rintoul and Wunsch, 1991). The Macdonald nominal layers at $24^{\circ} \mathrm{N}$ $\left(\sigma_{\mathrm{i}}\right)$ are given in the second column for reference. (Note that Macdonald changed the reference pressure when the potential density surface departed far from it. Her resulting layers consistently matched neutral surfaces, in contrast with the original $\sigma$ layers which occasionally led to large departure from neutral surfaces, e.g., a 500 dbar deviation was found near the Mediterranean waters.)

In the South Atlantic, the layer and water mass definitions were based on both Macdonald and Speer et al.(1996). The nominal interface between NADW and AABW, $\gamma^{n}=28.11$, remains close to $\theta=2^{\circ}$ between $30^{\circ} \mathrm{S}$ and $7^{\circ} \mathrm{N}$, but varies from $1.4^{\circ}$ to $1.8^{\circ}$ at $\mathrm{A} 11\left(45^{\circ} \mathrm{S}-30^{\circ} \mathrm{S}\right)$.

The initial Ekman transports derived from the NCEP re-analysis are given on the second column of Table 3.11 .

Conservation equations are applied as described in Chapter 1, and weighted according to Table C-2 (page 194). The "PO" conservation constraints were given lower weights in boxes "Rio", "Tristan da Cunha" and superbox A5-A9 because with the initial weight from Section 1.2.4 (page 22), the derived circulation contained mass residuals in deep layers and systematic negative diffusivities in several boxes. The net flux across all latitudes was constrained to the Bering Strait volume flux and salt fluxes. Coachman and Aagaard (1988) estimated the Bering Strait flow to $-0.8 \pm 0.6 \mathrm{~Sv}$ for the mass and $26.7 \times 10^{6} \mathrm{~kg} \mathrm{~s}^{-1}$ for the 
salt. However, the uncertainties applied on the net flux constraints across individual sections are much larger, according to the error budget (Table C-1, page 193). The Florida Strait transport was constrained to $31 \pm 1 \mathrm{~Sv}$, following the measurements of Schott et al. (1988a) ${ }^{2}$. Section A6 does not include the part of the North Brazil Current (NBC) that is on the continental shelf. Current meters deployed near $7^{\circ} \mathrm{N}$ produced an estimate of this transport of $4.8 \mathrm{~Sv}$ (Johns et al., 1998). Similarly to Lux (1997), the 5-Sv continental NBC was included in my tracer budgets by using the average tracer concentration in the first 100 dbars of the westernmost station. Because the shelf water is warm $\left(26^{\circ} \mathrm{C}\right)$, the influence of the NBC transport is important $(0.5 \mathrm{PW})$.

In the south, the AABW flux was constrained to $4 \pm 0.4 \mathrm{~Sv}$ northward in the Vema Channel (Hogg et al., 1982) and to $2.9 \pm 1 \mathrm{~Sv}$ in the Hunter Channel (Zenk et al., 1999). The total flux was constrained to $7 \pm 2 \mathrm{~Sv}$. Further North, the AABW flux was constrained to $5.5 \pm 2 \mathrm{~Sv}$ in the western part of the basin at $11^{\circ} \mathrm{S}$ (A8), according to McCartney and Curry (1993); to $3 \pm 6 \mathrm{~Sv}$ at $4.5^{\circ} \mathrm{S}(\mathrm{A} 7)$ and $2 \pm 4 \mathrm{~Sv}$ at $7.5^{\circ} \mathrm{N}(\mathrm{A} 6)$, in the western basin according to the current meter measurements made in the same basin but at the Equator by Hall et al. (1997). (There too, the weights were defined by the error budget.) The AABW flux through the Romanche and Chain Fracture zones was constrained to 1.4 $\pm 3.6 \mathrm{~Sv}$ (Mercier and Speer, 1998).

The Deep Western Boundary Current was weakly constrained at $7.5^{\circ} \mathrm{N}$ to $20 \pm 10 \mathrm{~Sv}$, according the SOFAR float measurements of Richardson and Schmitz (1993) (between 900 and $2800 \mathrm{dbars}$ and within $700 \mathrm{~km}$ of the coast). At $19^{\circ} \mathrm{S}$, the DWBC transport was constrained according to the current meter measurements of Weatherly et al. (1999), that is, $-15 \pm 7 \mathrm{~Sv}(1200-2000 \mathrm{dbars}) ;-11 \pm 4 \mathrm{~Sv}(2000-2800 \mathrm{dbars})$; and $-13 \pm 6 \mathrm{~Sv}$ (28003600 dbars). The "bathymetric constraints", essentially due to the Mid-Atlantic Ridge, are listed on Table 3.12 .

\footnotetext{
${ }^{2}$ An uncertainty of $\pm 1 \mathrm{~Sv}$ is lower than the one suggested in the preceeding error budget. But I kept this original uncertainty from independent measurements because, from the many estimates derived from hydrographic data in the Florida Strait, all seem to be consistent with the direct measurement to this accuracy.
} 


\begin{tabular}{|c|c|c|c|}
\hline Layer & $\begin{array}{l}\text { Upper } \\
\text { interface }\end{array}$ & $\begin{array}{c}24^{\circ} \mathrm{N} \text { (A5) Properties } \\
\sigma_{x} \text { from Macdonald (1998) }\end{array}$ & $\begin{array}{l}\text { Water mass } \\
\text { label }\end{array}$ \\
\hline 1 & surface & $\theta=17.9$ & Surface Water \\
\hline 2 & $\gamma^{n}=26.44$ & $\sigma_{\theta}=26.40 ; \theta=15.8$ & \multirow{3}{*}{$\begin{array}{c}\text { Thermocline } \\
\text { Water }\end{array}$} \\
\hline 3 & $\gamma^{n}=26.85$ & $\sigma_{\theta}=26.80 ; \theta=13.6$ & \\
\hline 4 & $\gamma^{n}=27.162$ & $\sigma_{\theta}=27.10 ; \theta=11.3$ & \\
\hline 5 & $\gamma^{n}=27.38$ & $\sigma_{\theta}=27.30 ; \theta=8.80$ & \multirow{2}{*}{$\begin{array}{c}\text { Intermediate } \\
\text { Water }\end{array}$} \\
\hline 6 & $\gamma^{n}=27.62$ & $\sigma_{0}=27.50 ; \theta=6.90$ & \\
\hline 7 & $\gamma^{n}=27.82$ & $\sigma_{0}=27.70 ; \theta=5.20$ & \multirow{8}{*}{ NADW } \\
\hline 8 & $\gamma^{n}=27.922$ & $\sigma_{2}=36.87 ; \theta=3.60$ & \\
\hline 9 & $\gamma^{n}=27.975$ & $\sigma_{2}=36.94 ; \theta=3.20$ & \\
\hline 10 & $\gamma^{n}=28.008$ & $\sigma_{2}=36.98 ; \theta=3.00$ & \\
\hline 11 & $\gamma^{n}=28.044$ & $\sigma_{2}=37.02 ; \theta=2.76$ & \\
\hline 12 & $\gamma^{n}=28.072$ & $\sigma_{4}=45.81 ; \theta=2.50$ & \\
\hline 13 & $\gamma^{n}=28.0986$ & $\sigma_{4}=45.85 ; \theta=2.24$ & \\
\hline 14 & $\gamma^{n}=28.11$ & $\sigma_{4}=45.87 ; \theta=2.11$ & \\
\hline 15 & $\gamma^{n}=28.1295$ & $\sigma_{4}=45.90 ; \theta=1.93$ & \multirow{3}{*}{$\begin{array}{l}\text { Bottom } \\
\text { Water }\end{array}$} \\
\hline 16 & $\gamma^{n}=28.141$ & $\sigma_{4}=45.91 ; \theta=1.85$ & \\
\hline 17 & $\gamma^{n}=28.154$ & $\sigma_{4}=45.92 ; \theta=1.81$ & \\
\hline
\end{tabular}

Table 3.9: North Atlantic Ocean neutral surface layers $\Gamma$ (in $\mathrm{kg} \mathrm{m}^{-3}$ ). The Macdonald (1998) water mass definition are indicated (from her Table 11). NADW stands for North Atlantic Deep Water.

\begin{tabular}{|c|c|c|c|}
\hline Layer & $\begin{array}{l}\text { Upper } \\
\text { interface }\end{array}$ & $11^{\circ} \mathrm{S}(\mathrm{A} 8)$ Temperature & $\begin{array}{c}\text { Water mass } \\
\text { label }\end{array}$ \\
\hline 1 & surface & $\theta=27$ & \multirow{3}{*}{$\begin{array}{l}\text { Surface } \\
\text { Water }\end{array}$} \\
\hline 2 & $\gamma^{n}=26.2$ & $\theta=16.6$ & \\
\hline 3 & $\gamma^{n}=26.8$ & $\theta=11.8$ & \\
\hline 4 & $\gamma^{n}=27.3$ & $\theta=6.20$ & AAIW \\
\hline 5 & $\gamma^{n}=27.5$ & $\theta=4.30$ & \multirow{3}{*}{$\begin{array}{l}\text { Upper } \\
\text { NADW }\end{array}$} \\
\hline 6 & $\gamma^{n}=27.72$ & $\theta=3.90$ & \\
\hline 7 & $\gamma^{n}=27.903$ & $\theta=3.70$ & \\
\hline 8 & $\gamma^{n}=27.97$ & $\theta=3.30$ & \multirow{2}{*}{$\begin{array}{l}\text { Middle } \\
\text { NADW }\end{array}$} \\
\hline 9 & $\gamma^{n}=28.03$ & $\theta=2.75$ & \\
\hline 10 & $\gamma^{n}=28.07$ & $\theta=2.40$ & Lower NADW \\
\hline 11 & $\gamma^{n}=28.11$ & $\theta=2.00$ & \multirow{6}{*}{ AABW } \\
\hline 12 & $\gamma^{n}=28.20$ & $\theta=1.44$ & \\
\hline 13 & $\gamma^{n}=28.23$ & $\theta=0.60$ & \\
\hline 14 & $\gamma^{n}=28.25$ & $\theta=0.40$ & \\
\hline 15 & $\gamma^{n}=28.27$ & $\theta=0.22$ & \\
\hline 16 & $\gamma^{n}=28.296$ & $\theta=0.11$ & \\
\hline
\end{tabular}

Table 3.10: South Atlantic Ocean neutral surface layers $\Gamma$ (in $\mathrm{kg} \mathrm{m}^{-3}$ ). Speer et al. (1996) layer definition are used. Water mass definition are indicated (from their Table 4). 


\begin{tabular}{|c|c|c|c|}
\hline Section & Initial & Final & \% correction \\
\hline A2 & $-2.7 \pm 1$ & $-2.7 \pm 1$ & $0 \%$ \\
\hline Florida Strait & 0 & 0 & - \\
\hline A5 & $+4.6 \pm 2$ & $3 \pm 1.7$ & $-32 \%$ \\
\hline A6 & $+8.2 \pm 4$ & $8.3 \pm 3.6$ & $1 \%$ \\
\hline A7 & $-17 \pm 8$ & $-19 \pm 6.8$ & $13 \%$ \\
\hline A8 & $-10 \pm 5$ & $-8.6 \pm 4$ & $-14 \%$ \\
\hline A9 & $-5.5 \pm 3$ & $-6.3 \pm 2.6$ & $13 \%$ \\
\hline A10 & $-0.5 \pm 1$ & $-0.6 \pm 1$ & - \\
\hline A11 & $+6 \pm 3$ & $5.8 \pm 2.8$ & $-3 \%$ \\
\hline
\end{tabular}

Table 3.11: Atlantic initial Ekman transport and correction

\begin{tabular}{|l|l|l|l|l|}
\hline Name & Section & Layer depth & Net flux & Longitude \\
\hline Walvis R. North & A10 & $\gamma^{n}=28.11(4000)$ & $0 \pm 1 \mathrm{~Sv}$ & $7.3^{\circ} \mathrm{W}-1.7^{\circ} \mathrm{E}$ \\
\hline Walvis R. South & A10 & $\gamma^{n}=28.11(4200)$ & $0 \pm 1 \mathrm{~Sv}$ & $2.2^{\circ} \mathrm{E}-13.4^{\circ} \mathrm{E}$ \\
\hline \hline Name & Box & Layer depth & Net Div. & Longitudes \\
\hline \hline East N. Atlantic & A2/A5 & $\gamma^{n}=28.0986(3500)$ & $0 \pm 3 \mathrm{~Sv}$ & $27^{\circ} \mathrm{W}-11^{\circ} \mathrm{W} / 45^{\circ} \mathrm{W}-16^{\circ} \mathrm{W}$ \\
\hline East S. Atlantic 1 & A8/A9 & $\gamma^{n}=28.11 \quad(4000)$ & $0 \pm 2 \mathrm{~Sv}$ & $13.2^{\circ} \mathrm{W}-13.4^{\circ} \mathrm{E} / 12.4^{\circ} \mathrm{W}-10.8^{\circ} \mathrm{E}$ \\
\hline East S. Atlantic 2 & A9/A10 & $\gamma^{n}=28.11 \quad(4000)$ & $0 \pm 2 \mathrm{~Sv}$ & $12.4^{\circ} \mathrm{W}-10.8^{\circ} \mathrm{E} / 13.6^{\circ} \mathrm{W}-14.7^{\circ} \mathrm{E}$ \\
\hline East S. Atlantic 3 & A10/A11 & $\gamma^{n}=28.20 \quad(4500)$ & $0 \pm 2 \mathrm{~Sv}$ & $13.6^{\circ} \mathrm{W}-14.7^{\circ} \mathrm{E} / 15^{\circ} \mathrm{W}-15.6^{\circ} \mathrm{E}$ \\
\hline
\end{tabular}

Table 3.12: Constraints imposed on the deep layer flux by bathymetric features. The constraints are imposed on either the net flux through a single section for a longitude range (upper part, positive northward) or on the net divergence (Div.) in a box between two sections (lower part). The layer and depth below which the flux is constrained are indicated along with the uncertainty on the net flux (section) or divergence (box) (in Sv). 


\begin{tabular}{|l|l|l|}
\hline Section & Reference surface & Remark \\
\hline \hline A2 & bottom (LCD) & match to Clarke et al. (1998) in the DWBC \\
\hline F1. Strait & bottom (LCD) & $25 \pm 20 \mathrm{~cm} \mathrm{~s}^{-1}$ initial (Schott et al., 1988a, Figure 2) \\
\hline A5 & $\gamma^{n}=28.1295 ; \theta \simeq 1.90^{\circ}$ & NADW $/$ AABW \\
\hline A6 & Pair-Specific & Lux (1997) \\
\hline A7 & Pair-Specific & \\
\hline A8 & Pair-Specific & Speer et al. (1996) \\
\hline A9 & Pair-Specific & Weatherly et al. (1999) \\
\hline A10 & $\gamma^{n}=28.11 ; \theta \simeq 1.90^{\circ}$ & NADW /AABW \\
\hline A11 & $\gamma^{n}=28.11 ; \theta \simeq 1.40^{\circ}-1.8^{\circ}$ & NADW /AABW - Saunders and King (1995) \\
\hline
\end{tabular}

Table 3.13: Reference surfaces in the Atlantic Ocean. LCD stands for Last Common Depth of station pairs. MAR=Mid-Atlantic Ridge.

\section{Reference surfaces and dianeutral terms}

The reference surfaces, indicated in Table 3.13, were chosen approximately at the interface between AABW and NADW, or according to current meter measurements or previous studies. At $19^{\circ} \mathrm{S}$, the reference surface was taken at the bottom in the first $300 \mathrm{~km}$ of the western end, based on the current meter measurements of Weatherly et al. (1999). Farther east, the reference was taken at 3000 dbars to avoid a high shear region below that depth west of the Mid-Atlantic Ridge. Still at $19^{\circ} \mathrm{S}$ but in the eastern Basin, the reference was taken at the bottom. Integrated fluxes after inversion were insensitive to this choice versus the $\gamma^{n}=28.11$ surface.

\subsubsection{Atlantic Ocean Circulation}

Except for the DWBC transport across $19^{\circ} \mathrm{S}$ between 2800 and 3000 dbars and the Romanche Fracture Zone transports, the standard model solution met all Atlantic constraints within one standard deviation of the a priori noise range. The model's lower DWBC transport at $19^{\circ} \mathrm{S}$ was found to be weaker than the Weatherly et al. (1999) constraint (3Sv southward), but still within 2 standard deviations. Mass residuals are indicated on Fig. 3-10. These are notable only in the upper 1000 dbars, but indistinguishable from zero everywhere. There is a little more noise in the deep layers of the Rio and Tristan da Cunha boxes (Fig. 3-10f, g), probably due to enhanced time variability there. The maximum adjustments in the net fluxes in the surface layer from the initial guess (including 
the Ekman transport) is in the $20 \%-30 \%$ range (Table 3.11 ).

Absolute velocities and transports integrated from the west are given in Appendix D (pages 220-235). The NADW flow does not appear to take place exclusively in a welldefined deep western boundary current across every section (e.g. at $24^{\circ} \mathrm{N}$, page 223 ), as

suggested by ocean circulation theory and current meter measurements. This was already pointed out by other authors who used different sections (Macdonald, 1998; Rintoul and Wunsch, 1991). It is not clear whether such an interior flow is an accident of a particular section time or reference surface choice. Nevertheless, in my model, net integrated property fluxes appear to be insensitive to the details of the horizontal circulation. That is, forcing the velocity field with results from a current meter array can change the horizontal structure as was done at A2, but does not necessarily affect the integrated fluxes.

The zonally integrated mass flux streamfunctions of the standard circulation are shown on Figure 3-11 for each section. The net fluxes (numbers below the plots) are indistinguishable from the Bering Strait outflow but are much more uncertain because of the baroclinic variability in the upper layers. Integrated transports over chosen sets of layers are indicated on Table 3.14. (The layers of integration do not correspond exactly to the approximate water masses defined above. Rather, the flow is summed over consecutive layers for which the integrated transport is flowing in the same direction.)

\section{AABW}

A net northward flux of $\mathrm{AABW}$ (defined as the total flow in the northward-flowing bottom layers) is indicated in the South Atlantic, gradually decreasing from $6 \pm 1 \mathrm{~Sv}$ at $30^{\circ} \mathrm{S}$ to $1 \pm 1 \mathrm{~Sv}$ at $24^{\circ} \mathrm{N}$. At $45^{\circ} \mathrm{S}$, the $\mathrm{AABW}$ flux is more uncertain with $5 \pm 2 \mathrm{~Sv}$. Within my error bars, the estimated transport in the North Atlantic is consistent with the Schmitz and McCartney (1993) circulation schematic (their Figure 13). In the South Atlantic, the AABW flow is slightly smaller than but fully consistent with that suggested by Hogg et al. (1999). 

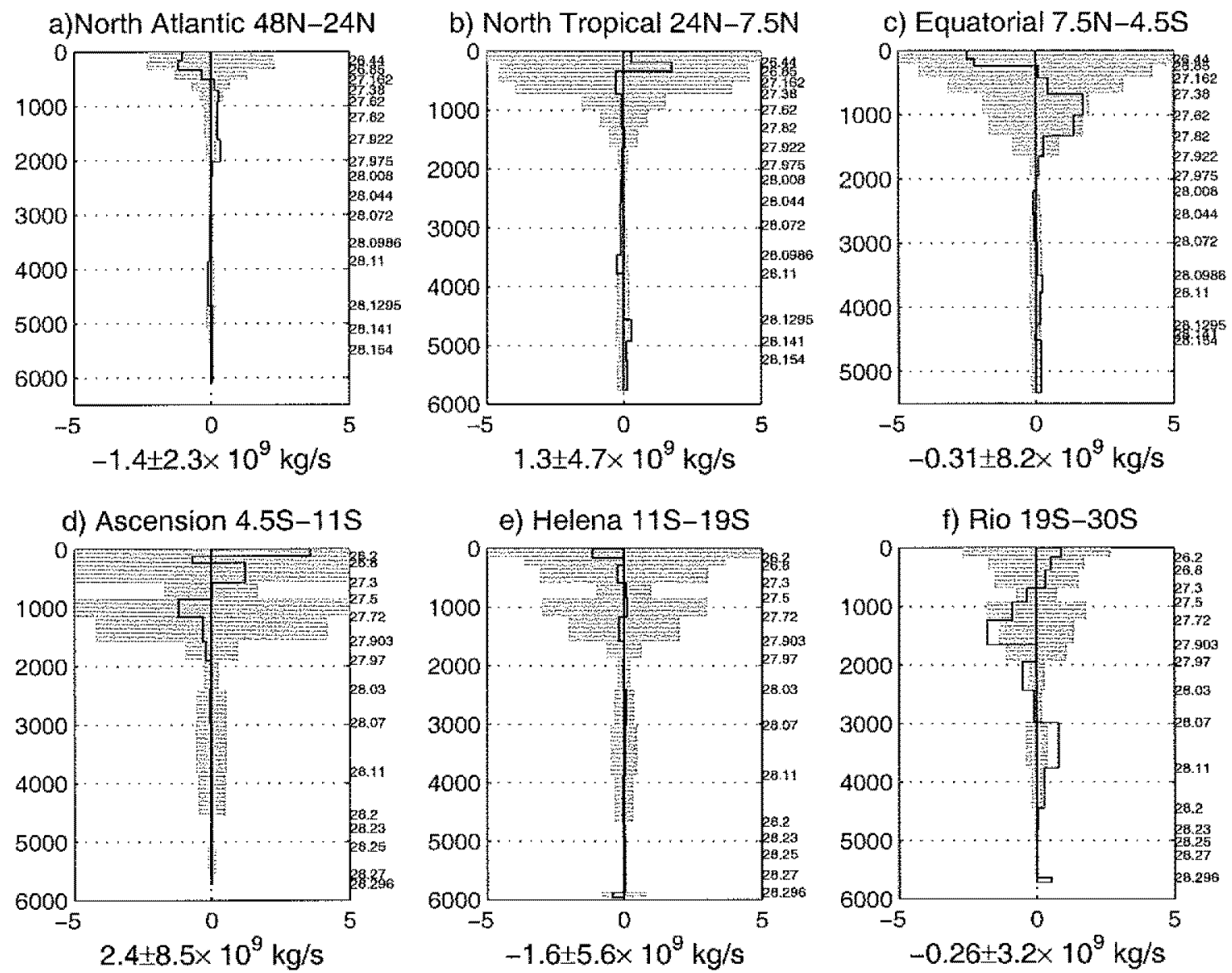

g) Tristan Da Cunha $305-45 \mathrm{~S}$

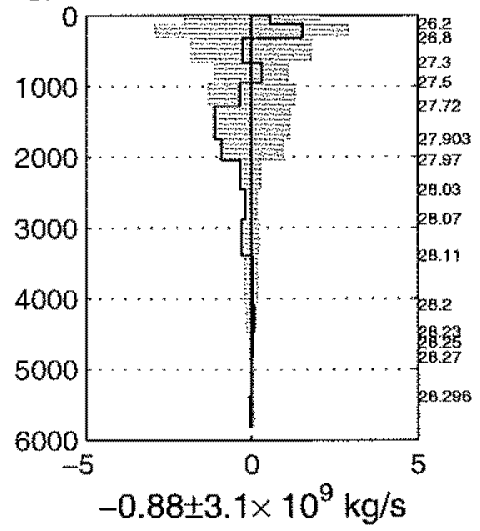

Figure 3-10: Standard solution mass residuals in the Atlantic Ocean. The line gives the residual in invidual layers (in $10^{9} \mathrm{~kg} \mathrm{~s}^{-1}$ ) and the shaded area give the one standard deviation uncertainty. The top-to-bottom residual is indicated below the graph. 
a) $\mathrm{A} 2(48 \mathrm{~N})$

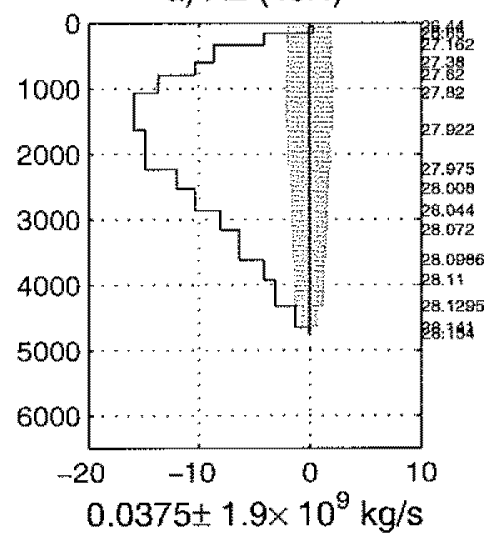

d) $A 6(7.5 \mathrm{~N})$

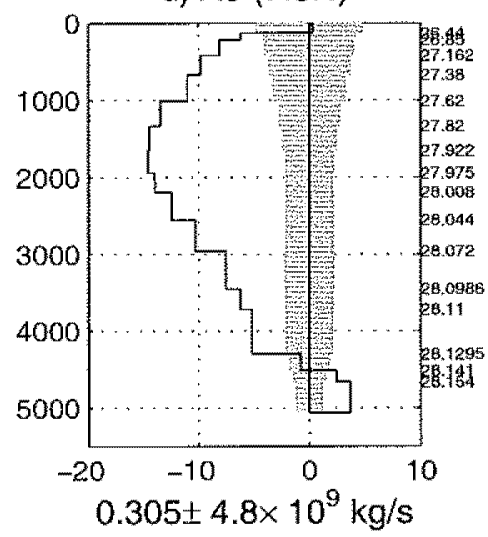

g) A9 (19S)

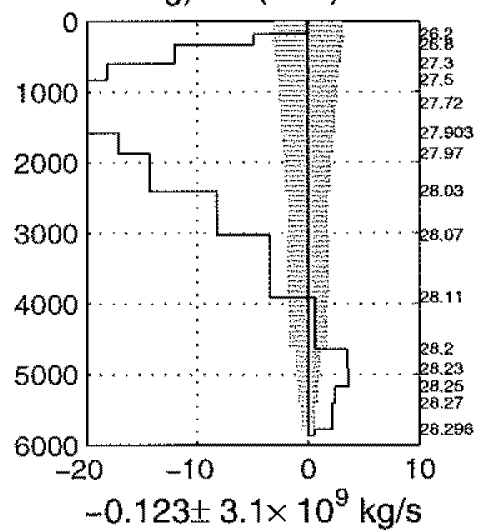

b) A5+Flor. St. $(24 \mathrm{~N})$

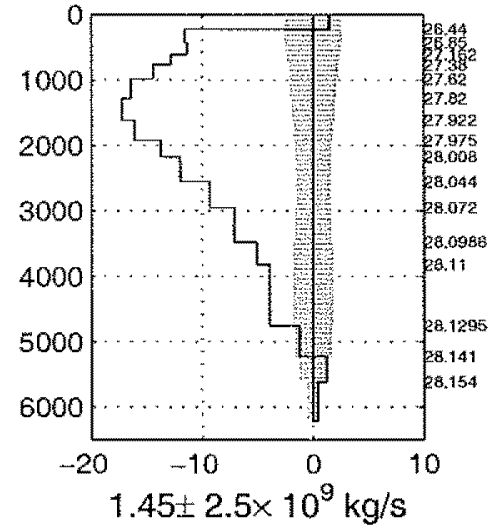

e) A7 (4.5S)

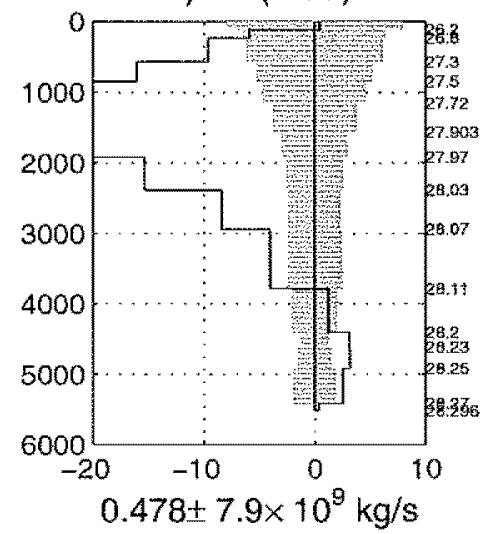

h) $\mathrm{A10}(30 \mathrm{~S})$

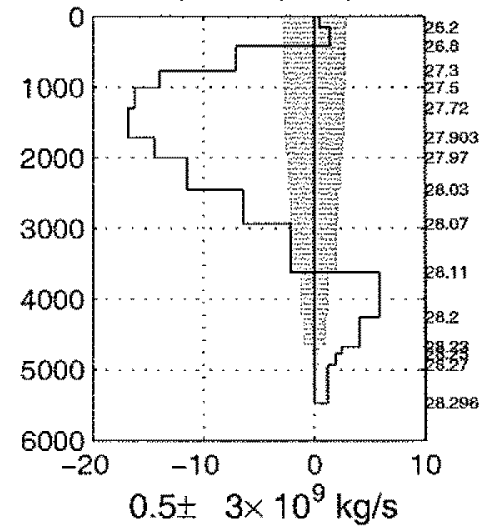

c) Florida.St.

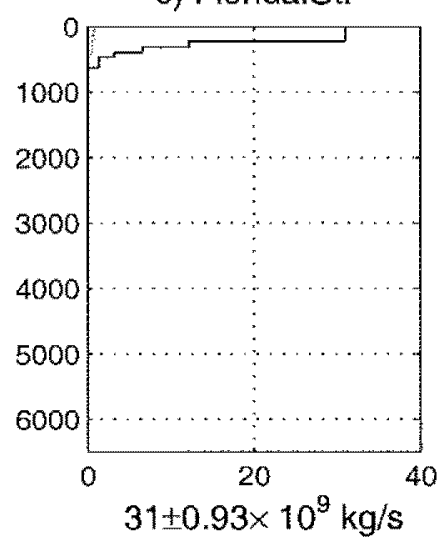

f) $A 8(11 S)$

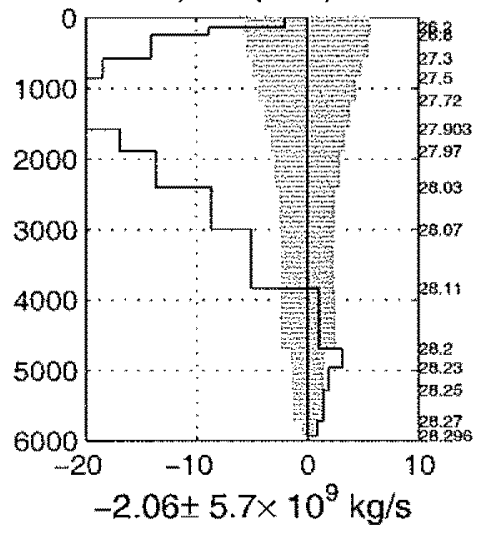

i) A11 (45S)

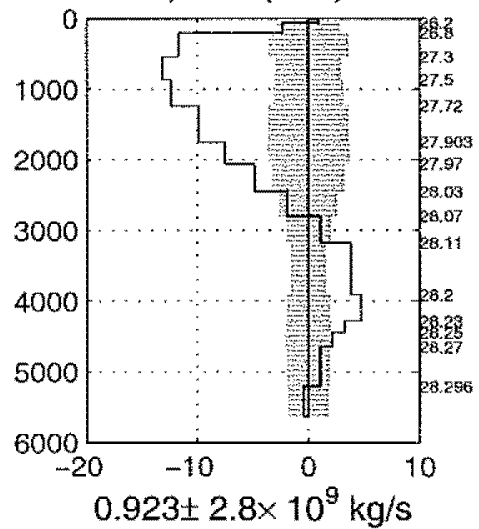

Figure 3-11: Atlantic Ocean standard circulation. The mass transport across each section is shown, integrated from the bottom (the overturning streamfunction). The shaded area corresponds to the uncertainty (one standard deviation). The net transport is indicated below each graph. While panel c) gives the total transport in the Florida strait alone, panel d) gives the total transport across $24^{\circ} \mathrm{N}$. Note the different scales on c). 


\begin{tabular}{|c|c|c|c|}
\hline Latitude & "AABW" & "NADW" & "Intermediate+thermocline" \\
\hline $48^{\circ} \mathrm{N}(\mathrm{A} 2)$ & 0 & $-16 \pm 2$ & $19 \pm 2$ \\
\hline $24^{\circ} \mathrm{N}(\mathrm{A} 5)$ & $1 \pm 1$ & $-18.5 \pm 2$ & $14 \pm 2$ \\
\hline $7.5^{\circ} \mathrm{N}(\mathrm{A} 6)$ & $3.6 \pm 1.2$ & $-18 \pm 3$ & $1 \pm 4$ \\
\hline $4.5^{\circ} \mathrm{S}(\mathrm{A} 7)$ & $3.4 \pm 1.2$ & $-29 \pm 4$ & $42 \pm 7$ \\
\hline $11^{\circ} \mathrm{S}(\mathrm{A} 8)$ & $3.1 \pm 1.5$ & $-23 \pm 4$ & $28 \pm 4$ \\
\hline $19^{\circ} \mathrm{S}(\mathrm{A} 9)$ & $3.4 \pm 1.1$ & $-23 \pm 3$ & $26 \pm 3$ \\
\hline $30^{\circ} \mathrm{S}(\mathrm{A} 10)$ & $6 \pm 1.3$ & $-23 \pm 3$ & $18 \pm 3$ \\
\hline $45^{\circ} \mathrm{S}(\mathrm{A} 11)$ & $5 \pm 2$ & $-18 \pm 4$ & $8 \pm 3$ \\
\hline
\end{tabular}

Table 3.14: Atlantic "water mass" transports (Sv). Transports are summed over consecutive layers of same integrated flow direction (see Fig.3-11), which does not correspond exactly to the approximate water mass definitions of Tables 3.9 and 3.10. Ekman transport is not included.

\section{NADW}

The NADW flow increases to the south, from $-16 \mathrm{~Sv}$ at $48^{\circ} \mathrm{N}$ to $-23 \mathrm{~Sv}$ at $30^{\circ} \mathrm{S}$ (Table 3.14 ). The increase in NADW transport can be explained by entrainment of AABW and AAIW (i.e., Schmitz and McCartney, 1993). The NADW flow at $45^{\circ} \mathrm{S}$ is marginally weaker and more uncertain, at $18 \pm 4 \mathrm{~Sv}$.

The present NADW transport estimates across the different sections are generally consistent with previous studies, e.g., Wunsch and Grant $\left(1982,14 \mathrm{~Sv}\right.$ at $48^{\circ} \mathrm{N} ; 18 \mathrm{~Sv}$ at $\left.24^{\circ} \mathrm{N}\right)$; Roemmich and Wunsch $\left(1985,24^{\circ} \mathrm{N},-20 \pm 5 \mathrm{~Sv}\right) ; \mathrm{Fu}(1986$, South Atlantic, $-20 \mathrm{~Sv})$; Speer et al. $\left(1996,-14 \mathrm{~Sv}\right.$ between $\gamma^{n}=27.5$ and $\gamma^{n}=28.11$ at $11^{\circ} \mathrm{S}$, versus $-20 \pm 4 \mathrm{~Sv}$ here); Holfort and Siedler $\left(1999,30^{\circ} \mathrm{S},-23 \mathrm{~Sv}\right)$; and Saunders and King (1995, $\left.45^{\circ} \mathrm{S},-22 \mathrm{~Sv}\right)$. Transports are also, within my error bars, relatively close to the Schmitz and McCartney (1993) and Schmitz(1996a) schematics.

Koltermann et al. (1999) estimated variable NADW transport at $48^{\circ} \mathrm{N}$ between -15 and $-20 \mathrm{~Sv}$ and at $24^{\circ} \mathrm{N}$ from -17 to $-24 \mathrm{~Sv}$. But the model that they used relied upon Sverdrup balance in the interior to compute the reference velocity adjustment. Luyten et al. (1985) suggested that Sverdrup balance was not a good approximation in the northern Atlantic. It is additionally difficult to distinguish between the parts of their circulation that is due to model configuration (e.g., reference surface choice) and the parts due to actual variability in the density field. 
The NADW transports agree quantitatively with the global Macdonald (1998) estimates everywhere except at $48^{\circ} \mathrm{N}$ and $11^{\circ} \mathrm{S}$. At $48^{\circ} \mathrm{N}$, Macdonald's large NADW transport of $-26 \mathrm{~Sv}$ could be due to temporal variability (e.g., Koltermann, 1999) or to a bottom triangle problem. (Transports are sensitive to the bottom triangles across this section when the reference surface is taken at the deepest common level of station pairs, see Figure 2-9, page 53). At $11^{\circ} \mathrm{S}$, I do not know what is responsible for Macdonald's large $-28 \pm 1 \mathrm{~Sv}$ transport-possibly time variability. Nevertheless, her high transport figure is within the present uncertainties.

At $4.5^{\circ} \mathrm{S}$, the NADW flux is the largest (29 $\left.\pm 4 \mathrm{~Sv}\right)$. From this same section, Lux (1997) obtained a similar flow associated with large dianeutral transfers between $7.5^{\circ} \mathrm{N}$ and $4.5^{\circ} \mathrm{S}$ that accounted for the difference in the overturning structure between the two latitudes. Geostrophic transports computed at $4.5^{\circ} \mathrm{S}$ are most subject to noise because of the small Coriolis parameter there, i.e., Chapter 2).

I did not attempt to compare in detail the deep flow with Reid (1994) because he gives transport values integrated from the upper limit of NADW all the way to the bottom, thus including AABW. But his qualitative picture of the circulation, with a large production of NADW (29 Sv flowing South at $60^{\circ} \mathrm{N}$ ), and a decrease of NADW flow to the south clearly conflicts with the standard circulation.

\section{Intermediate and surface flow}

Integrated intermediate and surface flows are indicated in Table 3.14, third column (Ekman transport is not included). Discussion of those flows is, by conservation of mass, equivalent to discussing the combined NADW and AABW transport. The total northward flow in the upper layers, including the Ekman flux, is in the range of 14 to $20 \mathrm{~Sv}$ outside the equatorial box, thus compatible with most authors' estimates, including Macdonald (1998, Tables 12 and 14); Schmitz and McCartney (1993) and Schmitz (1996a). The vertical structure of the flow (Fig. 3-11) generally indicates an equipartition between AAIW and surface flow, contrary to the results of de las Heras and Schlitzer (1999), who suggest that intermediate water flow dominates the return flow into the North Atlantic. 


\section{Boundary Currents}

The horizontal flow structure in the density classes defining the different water masses defined in Tables 3.9-3.10 is given in Appendix D (Figures D-26-D-40). Stations with common flow direction were selected to estimate boundary current transports. Again, the formal uncertainty of the boundary current transports is underestimated because the error budget did not take into account baroclinic variability on small scales.

In the North Atlantic at $48^{\circ} \mathrm{N}$, there is a northward flow between $50^{\circ} \mathrm{W}$ and $43^{\circ} \mathrm{W}$ of $47 \pm 2 \mathrm{~Sv}$ of waters above $\gamma^{n}=27.82\left(\theta=5^{\circ}\right)$. This flow, composed of the North Atlantic Drift plus recirculating currents, is larger than the Macdonald (1998) estimate (about $28 \mathrm{~Sv}$ from her Fig. 5) and the Schmitz and McCartney (1993) sketch that suggests $37 \mathrm{~Sv}$.

At $24^{\circ} \mathrm{N}$ above $\gamma^{n}=27.82\left(\theta=5.4^{\circ}\right)$, the upper part of the Antilles Current flows against the Bahamas Bank, with $27 \pm 3 \mathrm{~Sv}$ between $72^{\circ} \mathrm{W}$ and the Bank. This flow is more than twice that estimated by Macdonald (1998) and Schmitz and McCartney (1993), but is present in the baroclinic field before inversion. Current meter observations to the north at $26.5^{\circ} \mathrm{N}$ indicate a flux of about $5 \mathrm{~Sv}$ (Lee et al., 1996), but over a different longitude range (to my knowledge, there are no current meter data along the western part of the $24^{\circ} \mathrm{N}$ WOCE section).

The A6 section ( $6^{\circ} \mathrm{N}$ in the western end) is extremely noisy against the coast of Brazil. A northward flow of $5 \pm 1.5 \mathrm{~Sv}$ is found through the first station pair, that totals $10 \mathrm{~Sv}$ when the North Brazil Current on the shelf is included. But east of the first station pair, the eddy field dominates the transport signal, with transport variations of $\pm 8 \mathrm{~Sv}$ from one station to the next (above $\gamma^{n}=27.82, \theta=4.5^{\circ}$ ). At $11^{\circ} \mathrm{N}$, Macdonald (1998) obtained a northward flow (27 Sv) against the coast. But at that latitude, the North Brazil Current lies against the coast, in contrast with the highly variable retroflexion region at $6^{\circ} \mathrm{N}$ (Johns et al. 1990).

Farther south along the coast, the North Brazil Current can be identified with $44 \pm 7 \mathrm{~Sv}$ flowing north at $4.5^{\circ} \mathrm{S}$ (above $\gamma^{n}=27.82, \theta=4.2^{\circ}$, west of $34^{\circ} \mathrm{W}$ ) and $18 \pm 4 \mathrm{~Sv}$ at $11^{\circ} \mathrm{S}$ (above $\gamma^{n}=27.5, \theta=3.9^{\circ}$, west of $34.7^{\circ} \mathrm{W}$ ). The transport estimate at $11^{\circ} \mathrm{S}$ is similar to that obtained by Speer et al. (1996) from the same section, but only half the $40 \mathrm{~Sv}$ obtained by Macdonald (1998) from earlier data. 
At $19^{\circ} \mathrm{S}$ and $30^{\circ} \mathrm{S}$, one finds the southward-flowing Brazil Current, with a strength of $-15 \pm 3 \mathrm{~Sv}$ at $19^{\circ} \mathrm{S}$ (above $\gamma^{n}=27.5, \theta=3.6^{\circ}$, west of $36.9^{\circ} \mathrm{W}$ ) and $-14 \pm 4 \mathrm{~Sv}$ at $30^{\circ} \mathrm{S}$ (above $\gamma^{n}=27.5, \theta=3.5^{\circ}$, west of $40^{\circ} \mathrm{W}$ ), or $-9 \pm 3$ Sv west of $46.5^{\circ} \mathrm{W}$, depending upon the boundary current definition. Macdonald (1998) obtained a transport of similar magnitude at $23^{\circ} \mathrm{S}(-13 \pm 1 \mathrm{~Sv})$. My estimate at $30^{\circ} \mathrm{S}$ is lower than the current meter results of Muller et al. $\left(1998,-16 \mathrm{~Sv}\right.$ at $30^{\circ} \mathrm{S}$ and west of $\left.45^{\circ} \mathrm{W}\right)$ and Zemba $(1991,-22 \mathrm{~Sv}$ against the coast, based on earlier hydrographic data). It is not clear whether the difference is significant. There is little correction to the initial (relative) flow field in this region (Fig. D-38), so the difference with Zemba likely reflects real oceanic variability. At $45^{\circ} \mathrm{S}$, one identifies the Falkland Current, with $30 \pm 10 \mathrm{~Sv}$ flowing north (top-to-bottom, west of $58.4^{\circ} \mathrm{W}$ ), consistent with Saunders and King $(1995,40 \mathrm{~Sv})$.

Along the eastern boundary in the South Atlantic, the Benguela Current flows northward at $30^{\circ} \mathrm{S}$ (A10 and A11). Across A10, the strength of the flow is of $14 \pm 2 \mathrm{~Sv}$ east of $11.8^{\circ} \mathrm{E}$. Across A11, it is of $18 \pm 1.6 \mathrm{~Sv}$ east of $12.3^{\circ} \mathrm{E}$ (both above $\gamma^{n}=27.5, \theta=3.5^{\circ}$, that is, including AAIW). This magnitude is in agreement with independent estimates (e.g., Shannon and Nelson, $1996,15 \mathrm{~Sv}$ at $30^{\circ} \mathrm{S}$; Macdonald, $23 \pm 10 \mathrm{~Sv}$ at $27^{\circ} \mathrm{S}$, Garzoli et al., $1996,16 \mathrm{~Sv}$ at $\left.30^{\circ} \mathrm{S}\right)$.

In the South Atlantic, the present boundary current estimates are thus in agreement with estimates from the literature that are based on the same section or on modern current meter arrays. However in the North Atlantic, substantial differences with previous results are found. The differences may be simply due to changes in the circulation structure due to natural oceanic variability. But again the actual transport uncertainties (particularly those due to time variability) are much larger than the ones quoted here because of unaccounted baroclinic variability on small scales and the intense eddy field in western boundary regions that makes transport estimates extremely sensitive to the choice of integration boundaries.

\section{Dianeutral transfers and diffusivity}

Dianeutral transfers are shown on Fig. 3-12, upper panels. Upwelling in the bottom layers is found almost everywhere. At deep levels, upwelling is found mainly in the Equatorial $\left(4.5^{\circ} \mathrm{S}-7.5^{\circ} \mathrm{N}\right)$, Rio $\left(30^{\circ} \mathrm{S}-19^{\circ} \mathrm{S}\right)$ and Tristan da Cunha $\left(45^{\circ} \mathrm{S}-30^{\circ} \mathrm{S}\right)$ boxes, totaling $5-7 \mathrm{~Sv}$, 
a) North Atlantic $W^{*}$
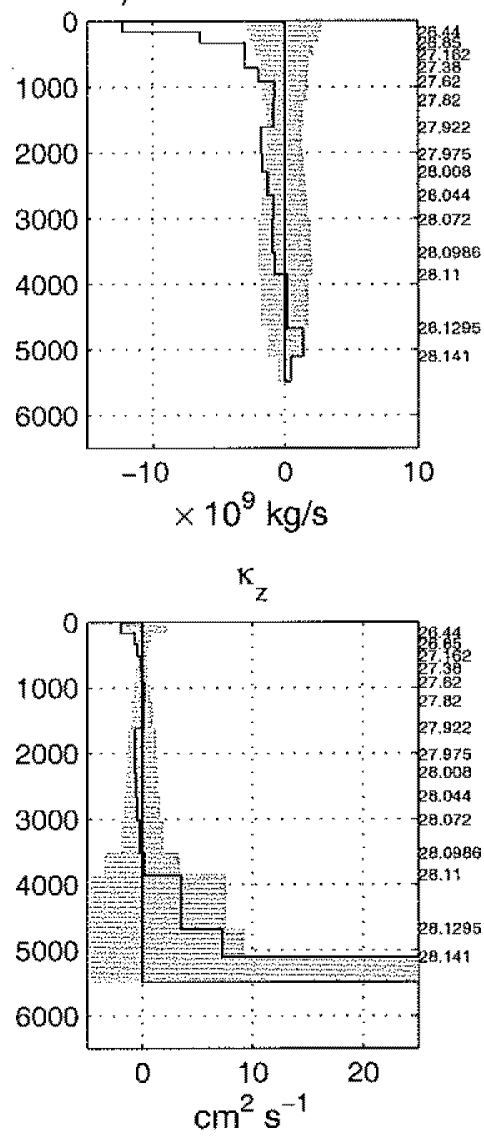

b) North Tropical W*
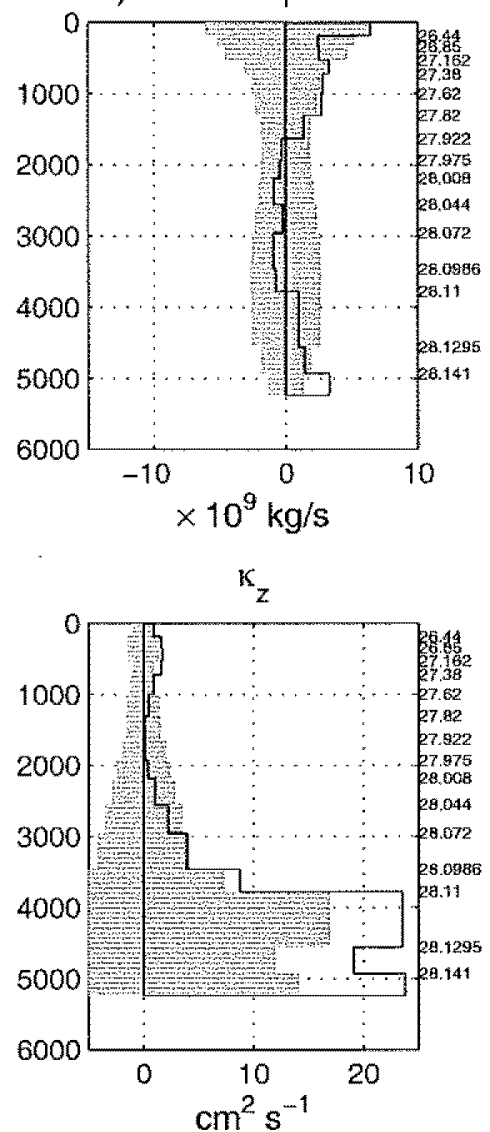
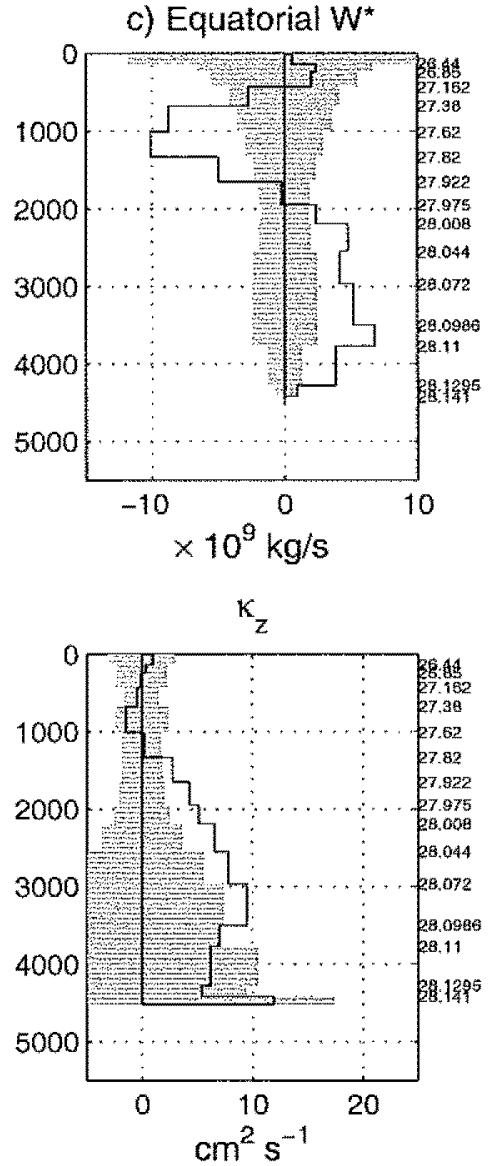

Figure 3-12: Dianeutral transfers (upper) and diffusivities (lower) in the Atlantic Ocean. The line indicates the transfer or diffusivity at the bottom of the layer. 
d) Helena $W^{*}$
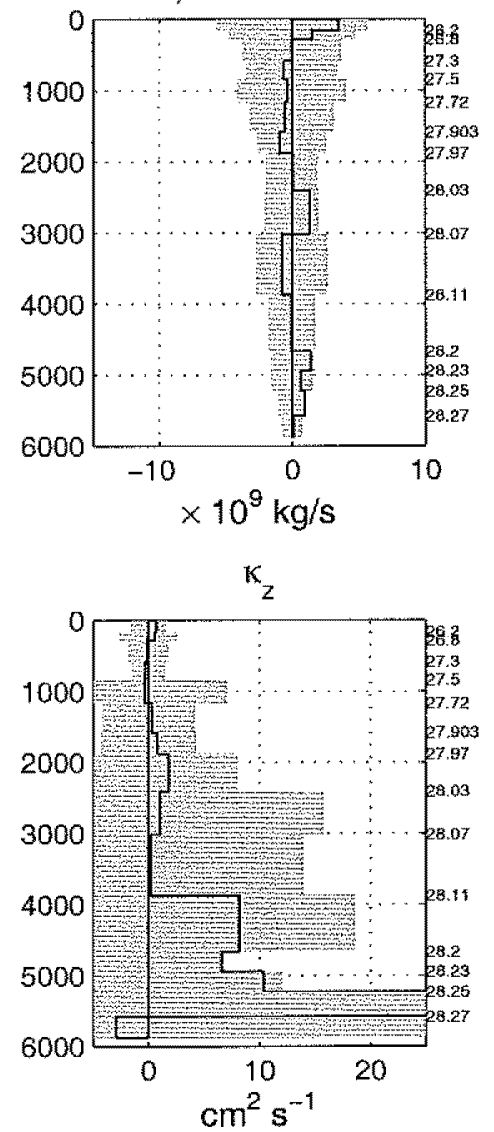

e) Rio $W^{*}$
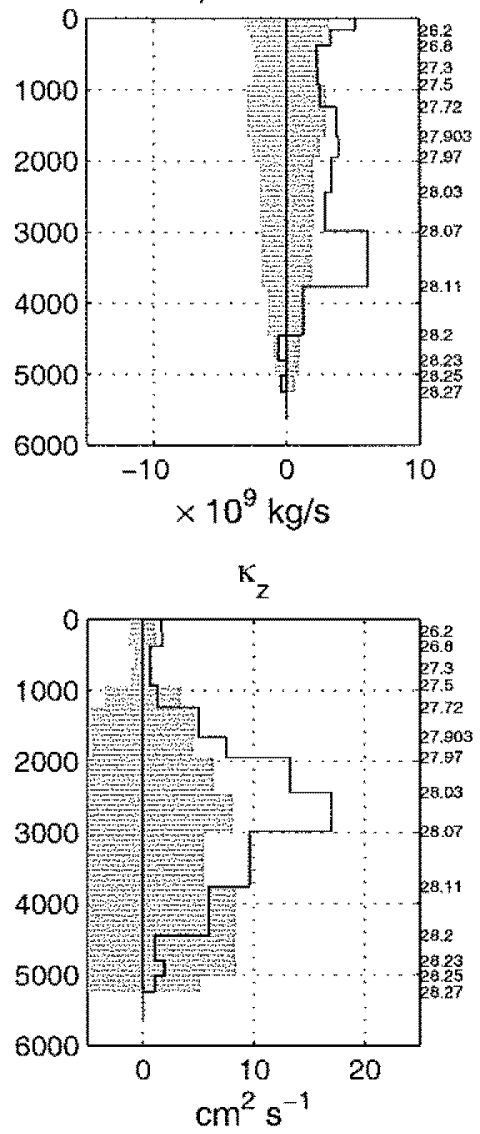

Figure 3-12: (continued)
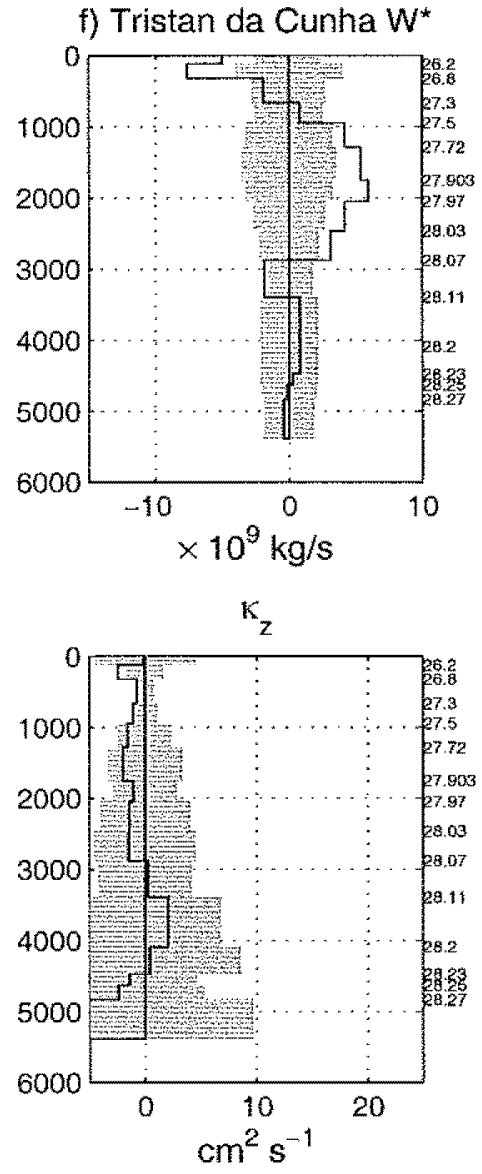
depending upon depth. At intermediate levels (1000-1500 dbars), upwelling is found in the Tristan da Cunha $\left(45^{\circ} \mathrm{S}-30^{\circ} \mathrm{S}\right.$, Fig.3-12f) box while downwelling is found in the Equatorial box, with $-10 \pm 2 \mathrm{~Sv}$ at $1300 \mathrm{dbars}$. This pattern is consistent with the NADW southward increase from entrainment of AABW and to some extent AAIW. In the other boxes, transfers are more uncertain at intermediate and deep levels.

In the surface layers, a large downwelling is found over the North Atlantic box, with $-13 \pm 3 \mathrm{~Sv}$, then upwelling in most other boxes, except in the Tristan da Cunha box, $-5 \pm$ 2 Sv downwelling. In the North Tropical and Rio boxes, the Ekman transport convergence would suggest a downwelling (in Cartesian coordinates). The geostrophic flux in the upper layer and possibly horizontal advection across outcropping isopycnals are thus strong enough to cancel and reverse this tendency.

Similarly, Macdonald (1998) found a consistent upwelling over the deep Equatorial layers and downwelling of AAIW. In the South Atlantic, her results are very noisy while in the North Atlantic she obtained upwelling, where my estimate does not produce significant dianeutral transfers.

Dianeutral diffusivities in the deep layers are positive and systematic in most boxes (Fig. 3-12, lower panels). Their values are in the range $0-50 \mathrm{~cm}^{2} \mathrm{~s}^{-1}$, depending upon depth and location. The highest diffusivities are found in the bottom layers (up to $50 \pm 50 \mathrm{~cm}^{2} \mathrm{~s}^{-1}$ in the North Atlantic), where the area of the layer interfaces are small-and subject to uncertainties in their calculation from Levitus (1994). In the North Tropical, Equatorial and Rio boxes, diffusivities are significant and in the range $5-20 \mathrm{~cm}^{2} \mathrm{~s}^{-1}$. The average diffusivity in the Rio box is of $4.6 \pm 3 \mathrm{~cm}^{2} \mathrm{~s}^{-1}$, which is similar to the $1-5 \mathrm{~cm}^{2} \mathrm{~s}^{-1}$ calculated by Hogg et al. (1982; 1999a) from a heat budget in the deep Brazil Basin. In the Equatorial and Rio boxes, highest diffusivities are found approximately at the depth of the crest of the Mid-Atlantic Ridge, over which the sharp topography is known to induce enhanced mixing (Polzin et al., 1997).

Fig. 3-13 shows the average diffusivities and dianeutral transfers in the central Atlantic superbox between $24^{\circ} \mathrm{N}$ and $19^{\circ} \mathrm{S}$ (see above). The average diffusivity in this superbox below $1000 \mathrm{dbars}$ is $7 \pm 2 \mathrm{~cm}^{2} \mathrm{~s}^{-1}$ ). There is no evidence for enhanced dianeutral mixing above 1000 dbars in the Atlantic Ocean; estimates are within the range allowed by the 

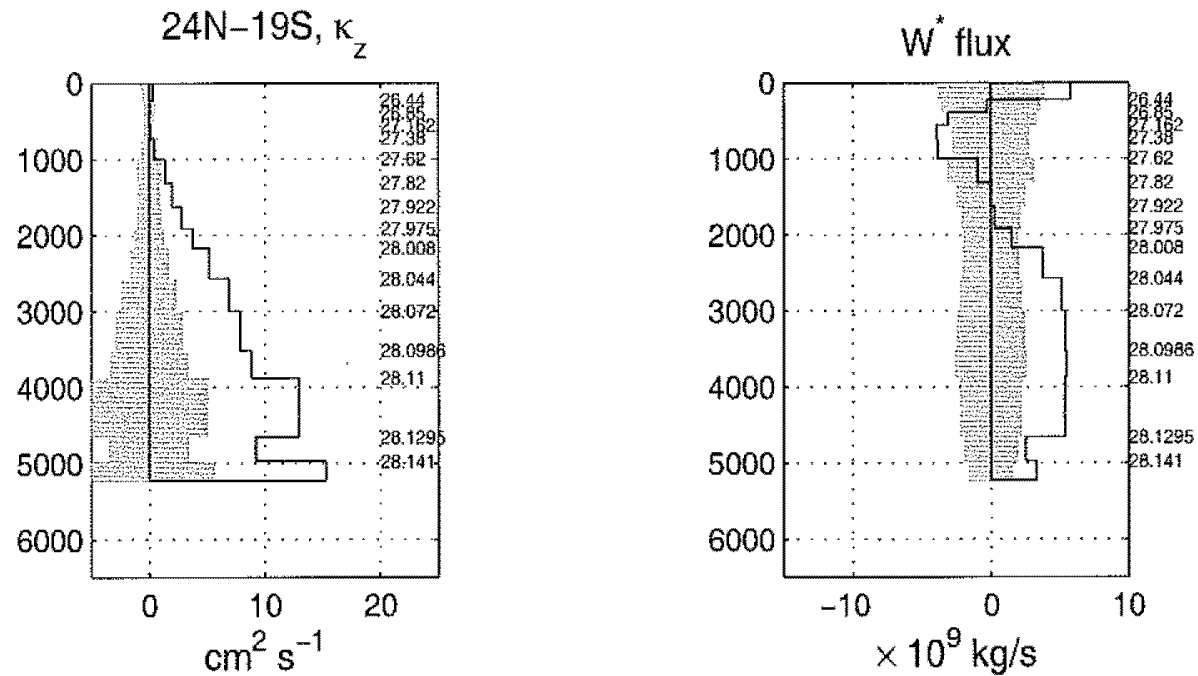

Figure 3-13: Diffusivities (left) and dianeutral transfers (right) in the central Atlantic box $\left(24^{\circ} \mathrm{N}-19^{\circ} \mathrm{S}\right)$.

uncertainties less than $1-2 \mathrm{~cm}^{2} \mathrm{~s}^{-1}$.

Dianeutral velocities and diffusivities are thus well resolved in the Atlantic, with average values for the diffusivities generally within the range of those previously reported. Basin-average diffusivities and dianeutral velocities are given at the end of this Chapter (page 138).

\subsubsection{Atlantic circulation summary}

The Atlantic circulation estimate is relatively well determined, compared with the larger uncertainties in other oceans. In particular, uncertainties of derived water mass are relatively small. North Atlantic Deep Water flows southward at $-16 \pm 2 \mathrm{~Sv}$ at $48^{\circ} \mathrm{N}$; it entrains Antarctic Bottom Waters and Antaretic Intermediate Waters on its way to the south, resulting is a flux of $-23 \pm 3 \mathrm{~Sv}$ at $30^{\circ} \mathrm{S}$. A northward flux of $6 \pm 1.3 \mathrm{~Sv}$ of Antarctic Bottom Waters is found to enter the Atlantic at $30^{\circ} \mathrm{S}$ west of the Mid Atlantic Ridge, progressively decreasing to $1 \pm 1 \mathrm{~Sv}$ at $24^{\circ} \mathrm{N}$ and, $0 \mathrm{~Sv}$ at $48^{\circ} \mathrm{N}$. The flux at $45^{\circ} \mathrm{S}$ is slightly different, although indistinguishable within uncertainties from the $30^{\circ} \mathrm{S}$ flow. The circulation in the intermediate and upper layers provides the 14 to $17 \mathrm{~Sv}$ flow of warm water to balance the deep water export. This circulation is generally consistent with the existing literature. 
However, pointwise differences were found, both in integrated transports and horizontal structure. A large part of those differences is probably attributable to natural oceanic variability; but because of the different methods used, it is difficult to determine how much. A possible extension of this work is to produce, with the same model, an estimate of the circulation based on the earlier data.

The strength of the Benguela Current that I diagnosed has about the same magnitude as independent estimates but, as is the case for most boundary currents, its transport is very uncertain due to an enhanced eddy field. As a result, no attempt was made to distinguish between the provenance of warm and cold waters, i.e., "warm water path" versus "cold water path" (i.e., Gordon, 1986; Rintoul, 1991).

The horizontal structure of the flow was analyzed succinctly and shows that locally, the flow direction is highly variable and generally does not correspond to the one suggested by water property distribution. However, the direction of the integrated flow does correspond roughly to that suggested by traditional water mass analysis.

Consistent upwelling, with significantly non-zero diffusivities, were found in the deep layers, with $\kappa^{*}$ values in the range 2 to $5 \mathrm{~cm}^{2} \mathrm{~s}^{-1}$ over most of the Atlantic. Strongest mixing and upwelling occur over different depth ranges, depending upon the region. 


\subsection{The Southern Ocean}

\subsubsection{Introduction}

The Southern Ocean is the highway of the global circulation. In addition to the oneway eastward flow in the Antarctic Circumpolar Current of about $140 \mathrm{~Sv}$ (Whitworth et al., 1982), there are major interbasin exchanges between the Indian and Atlantic Oceans (e.g., Georgi and Toole, 1982; Gordon, 1986). Recent estimates of the Southern Ocean circulation from inverse calculations based on hydrographic sections include the work of Macdonald (1998, global), Sloyan and Rintoul(1999a,b, south of $\left.12^{\circ} \mathrm{S}\right)$; Gille (1999, southeastern Pacific); and Rintoul (1991, Southern Atlantic).

Because most layers are in contact with the atmosphere, the Southern Ocean is a place of important dianeutral transfers, that is, of water mass transformation (e.g., Macdonald, 1998; Sloyan and Rintoul, 1999a; Schmitz and McCartney, 1993). In the present data set, all layers that are found above $4000 \mathrm{dbars}$ north of $30^{\circ} \mathrm{S}$ outcrop in the Southern Ocean. In that view, a large part of the diapycnal transfers in the global ocean could take place in the layer outcropping regions in the Southern Ocean. The Antarctic Bottom Waters $(\mathrm{AABW})$, the densest waters in the global ocean, are formed along the shelf of Antarctica. The Weddell Sea has been in the past regarded as the main formation site. However, recent observations indicate formation in various places around Antarctica including the Amery Basin, Adélie Land, Ross Sea and western Weddell Sea (Fig. 3-14), e.g., Rintoul (1998); Sloyan and Rintoul (1999); Orsi et al. (1999) and references therein. However, the total amount of AABW being produced is poorly known. Existing literature cites a range of 7 to 20 Sv. But estimates based on either heat budgets (Gordon, 1975) or inverse models forced by existing surface flux estimates (Sloyan and Rintoul, 1999a,b) indicate a much higher production rate of $\mathrm{O}(40-50 \mathrm{~Sv})$. The bottom water production rate will be discussed in light of my global model results. 


\subsubsection{Model constraints}

\section{Data and treatment}

Figure 3-14 shows the Southern Ocean meridional lines, namely, A21 (Drake, Jan. 1990, Roether et al., 1993); A12 (May 1992, Lemke, 1994); I6 (Feb. 1996, Park et al. 1999); I9S (Dec. 1995, Hufford et al., 1997); P12 (Jan 1995, Rosenberg et al., 1997) and P14S (Jan. 1996, McTaggart and Johnson, 1997). The hydrographic data were provided by the PIs W.Roether (A21); P. Lemke (A12); Y.-H. Park, N. Metzl and C. Jeandel (I6); M. McCartney and A. Gordon (I9S); S. Rintoul (P12); J. Bullister and G. Johnson (P14S).

\section{Layers and constraints}

The Southern boxes (Fig. 3-14) are respectively the Georgia-Weddell box (A21-A11-A12); the South Agulhas box (A12-I6); the Southern Indian box (I6-I5-I9S); the Southern Australia box (I9S-P12); and the South Pacific box (P12-P6-A21). A box called the "Macquarie" box defined by P12, P14S and the western part of P6 was explored, but the conservation constraints were weaker there because P14S did not reach the Antarctic continent. Moreover, this box was not closed between P6 and New Zealand, leaving a possible leak down to 2000 dbars to the north. An additional super box was defined in the Southern Indian between A12-I5-I9S.

The neutral surfaces defining water masses were again based on the Macdonald (1998) isopycnal surfaces (Table 3.16). But in the Southern Ocean, the formal separation between AABW and CDW required a somewhat lighter neutral density, $\gamma^{n}=28.27$, that corresponds to the sill depth of Drake Passage (i.e., Orsi et al., 1999). The initial Ekman transports are relatively small (Table 3.16) and their uncertainty was given the lower bound of $\pm 1 \mathrm{~Sv}$.

Because of the possible freshwater input to most layers as they outcrop, the a priori noise in the salt anomaly equations was increased to $\pm(35 \mathrm{~g} / \mathrm{kg}) \times(1 \mathrm{~Sv})$. No heat and PO conservation constraints were imposed. Thus only mass and total silica had an effective influence. Consequently, the uncertainties on the diagnosed dianeutral transfers and mixing are large relative to other oceans. 

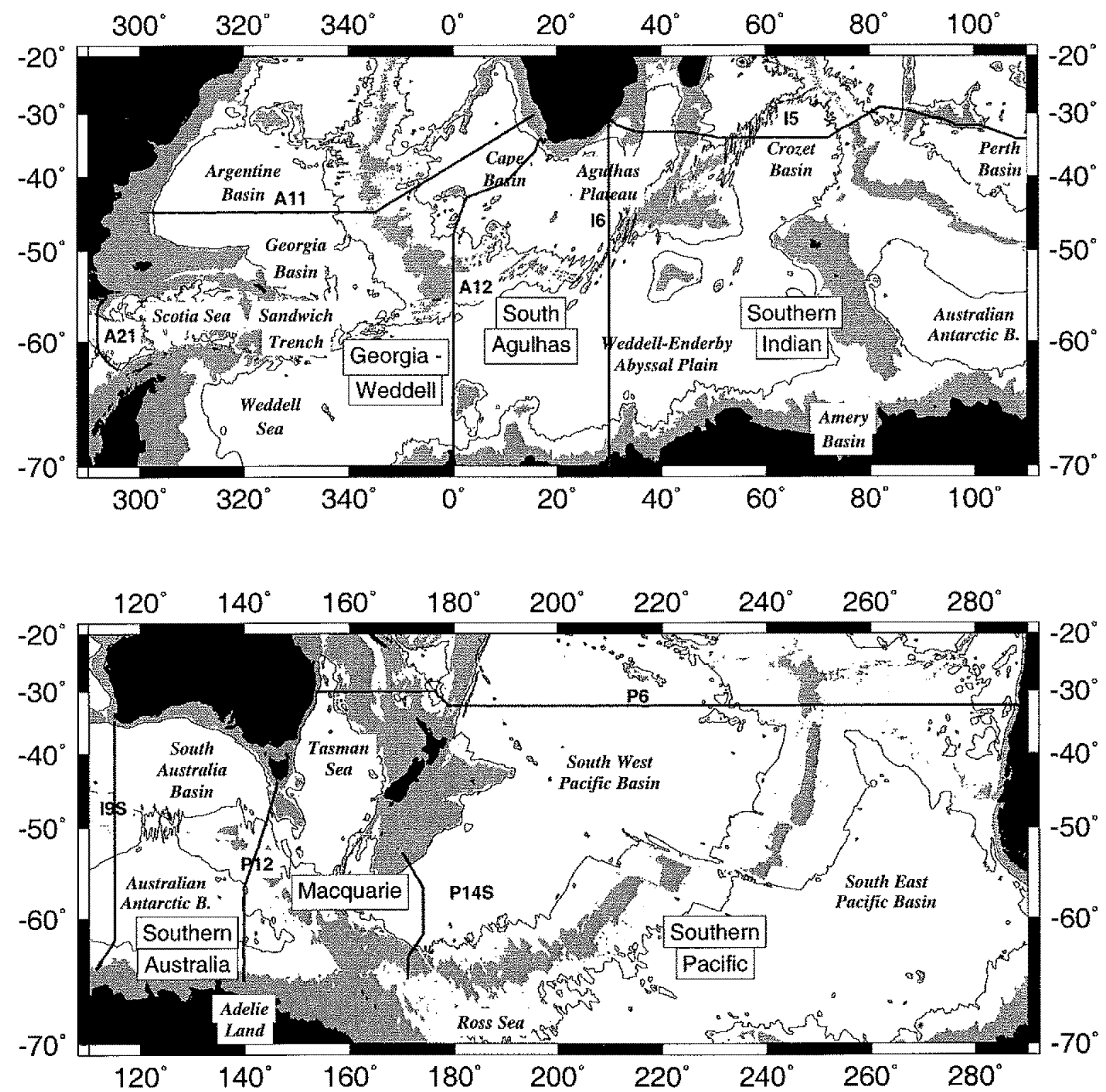

Figure 3-14: Southern Ocean sections and topography. The $4000 \mathrm{~m}$ isobath is contoured and areas shallower than $3000 \mathrm{~m}$ are shaded. Box names are indicated in the shaded rectangles. 


\begin{tabular}{|c|l|c|c|}
\hline Layer & $\begin{array}{l}\text { Upper } \\
\text { interface }\end{array}$ & $\begin{array}{c}0^{\circ} \mathrm{E}(\mathrm{A} 12) \text { Properties } \\
\theta=5.6^{\circ}\end{array}$ & $\begin{array}{c}\text { Water mass } \\
\text { name }\end{array}$ \\
\hline 1 & surface & $\theta=15^{\circ}$ & \multirow{2}{*}{ AArface Water } \\
\hline 2 & $\gamma^{n}=26.2$ & $\theta=10^{\circ}$ & \\
\hline 3 & $\gamma^{n}=26.8$ & $\theta=4.3^{\circ}$ & \multirow{2}{*}{ UCDW } \\
\hline 4 & $\gamma^{n}=27.32$ & $\theta=2.5^{\circ}$ & \\
\hline 5 & $\gamma^{n}=27.57$ & $\theta=1.7^{\circ}$ & \\
\hline 6 & $\gamma^{n}=27.72$ & $\theta=1.4^{\circ}$ & \multirow{2}{*}{ LCDW } \\
\hline 7 & $\gamma^{n}=27.903$ & $\theta=1.5^{\circ}$ & \\
\hline 8 & $\gamma^{n}=28.03$ & $\theta=1.45^{\circ}$ & \\
\hline 9 & $\gamma^{n}=28.07$ & $\theta=1^{\circ}$ & \multirow{2}{*}{ AABW } \\
\hline 10 & $\gamma^{n}=28.15$ & $\theta=0.6^{\circ}$ & \\
\hline 11 & $\gamma^{n}=28.20$ & $\theta=0.4^{\circ}$ & \\
\hline 12 & $\gamma^{n}=28.23$ & $\theta=0.15^{\circ}$ & \\
\hline 13 & $\gamma^{n}=28.25$ & $\theta=0^{\circ}$ & \\
\hline 14 & $\gamma^{n}=28.27$ & $\theta=-0.16^{\circ}$ & \\
\hline 15 & $\gamma^{n}=28.296$ & $\theta=-0.7^{\circ}$ & \\
\hline
\end{tabular}

Table 3.15: Southern Ocean neutral surface layers $\gamma^{n}$ (in $\mathrm{kg} \mathrm{m}^{-3}$ ). The apparent temperature reversal between layers 7 and 8 is due to outcrop and salinity effects. Water masses: AAIW=Antarctic Intermediate Waters; UCDW $/$ LCDW $=$ Upper/Lower Circumpolar Deep Waters. AABW=Antarctic Bottom Water.

The net flux through the Drake Passage (P21) was constrained to $140 \pm 6 \mathrm{~Sv}$ eastward, according to the current meter and hydrographic transport estimate of Whitworth et al. (1982) and the error budget of the preceding chapter. (140 $\pm 5 \mathrm{~Sv}$ corresponds to a weighted average of their estimates with respective uncertainties, i.e., Macdonald (1995); and an extra Sverdrup, rms, comes from the error budget.) The bathymetry in the Southern Ocean does not suggest imposing additional transport constraints.

\begin{tabular}{|c|c|c|c|}
\hline Section & Initial & Final & $\%$ correction \\
\hline A21 & $0.06 \pm 1$ & $0 \pm 1$ & - \\
\hline A12 & $-1.3 \pm 1$ & $-1.4 \pm 1$ & $1 \%$ \\
\hline I6 & $0.6 \pm 1$ & $0.6 \pm 1$ & $0 \%$ \\
\hline I9S & $-0.9 \pm 1$ & $-0.9 \pm 1$ & - \\
\hline P12 & $0 \pm 1$ & $0 \pm 0.5$ & - \\
\hline P14S & $-0.5 \pm 1$ & $-0.46 \pm 1$ & - \\
\hline
\end{tabular}

Table 3.16: Southern Ocean initial Ekman transport and correction (in Sv, positive eastward 


\section{Reference surfaces and dianeutral terms}

All reference surfaces were taken at the bottom (last common depths of station pairs), that is, where the velocitics are likely to be the weakest. As for the other oceans, a large range is allowed in the dianeutral transfers $\left( \pm 10^{-3} \mathrm{~cm} \mathrm{~s}^{-1}\right.$ and $\left.\pm 100 \mathrm{~cm}^{2} \mathrm{~s}^{-1}\right)$ because little is known about their actual size for outcropping layers. Moreover, the diffusivity formalism may not appropriately represent water mass modification by air-sea exchange.

\subsubsection{Southern Ocean Circulation}

All residuals of the equations of the "standard" solution were within the a priori range. Mass residuals in individual layers are tiny and all are indistinguishable from zero (Fig. 3$15)$. But the uncertainties of the residuals are close to the a priori values, reflecting the low weight of the salt equations and the absence of heat and PO constraints. Residuals in the Macquarie box indicate a systematic convergence, with $-5 \pm 7 \mathrm{~Sv}$ in total, possibly due to an eastward flux between P14S and Antarctica (or just noise). Because the wind is nearly perpendicular to the sections, the Ekman transports (and their corrections) play a minor role in the Southern Ocean (Table 3.16).

Absolute velocities and water-mass transports cumulated from the north are given in Appendix D (pages 236-247) for reference. The integrated flow for the standard solution of the Southern Ocean circulation is shown on Figure 3-16. The net fluxes through the "choke point" sections are close to the Drake Passage flow, $140 \pm 6$ Sv eastward across A21. South of Australia, the net flux is increased by about the size of the Pacific-Indonesian throughflow, adding up to $154 \pm 9 \mathrm{~Sv}$, which is similar to the regional calculation of Rintoul and Bullister (1999). Above 3000 dbars, the transports increase in a quasi-linear way, giving a parabolic shape to the streamfunction. Macdonald (1998) obtained a similar structure based on earlier data.

\section{Dianeutral transfers and diffusivity}

Dianeutral mass transfers are indicated on Fig.3-17. (In the Weddell region, the first six layers outcrop north of the ACC.) Because of the low property conservation weights, 

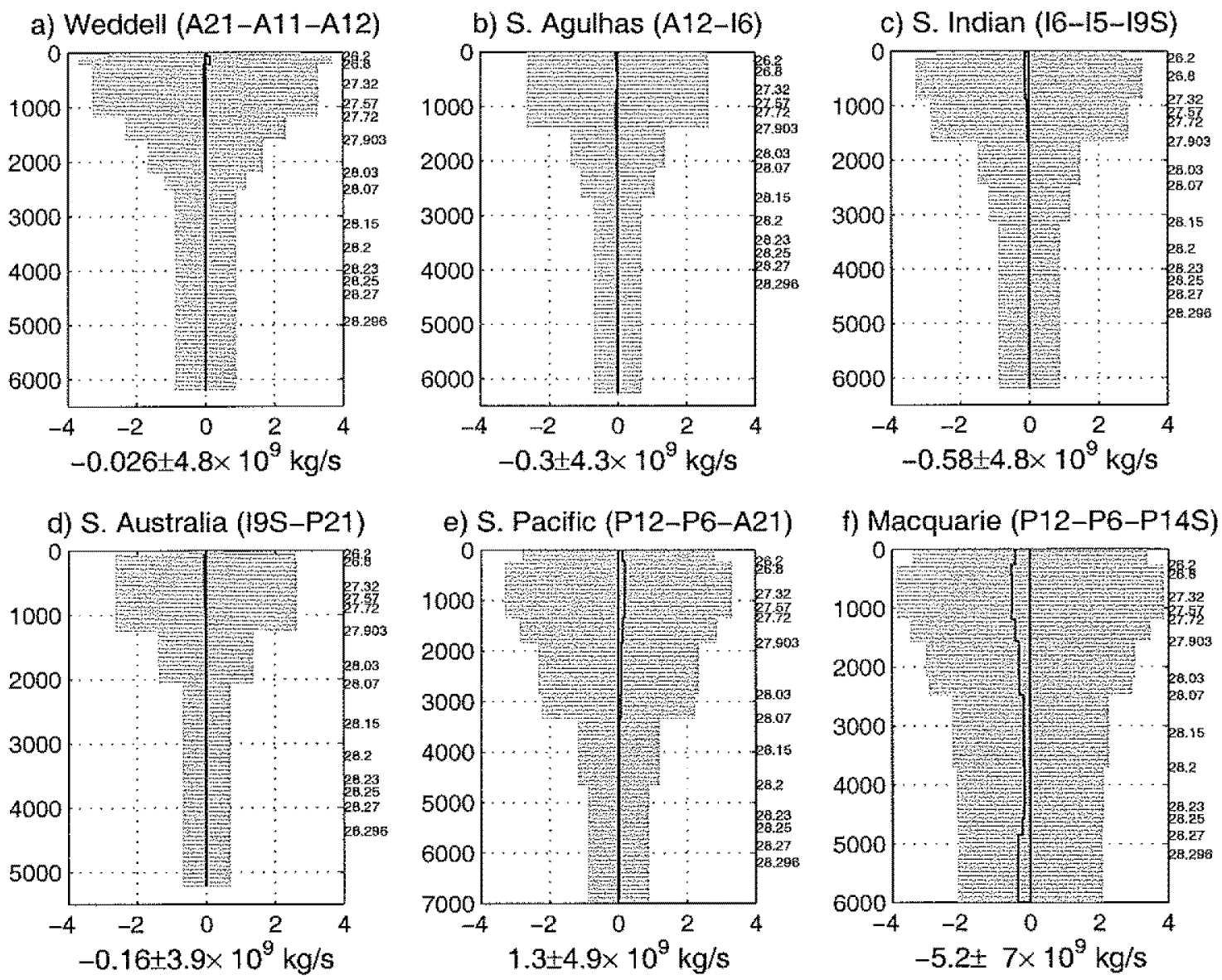

Figure 3-15: Mass residuals in the Southern Ocean boxes. 
a) A21 (Drake)

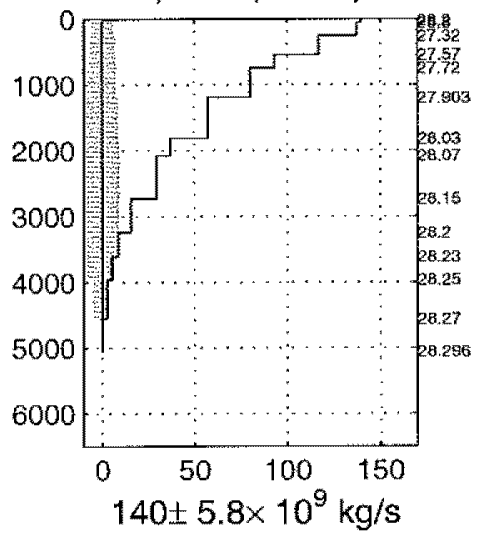

d) I9S (115E)

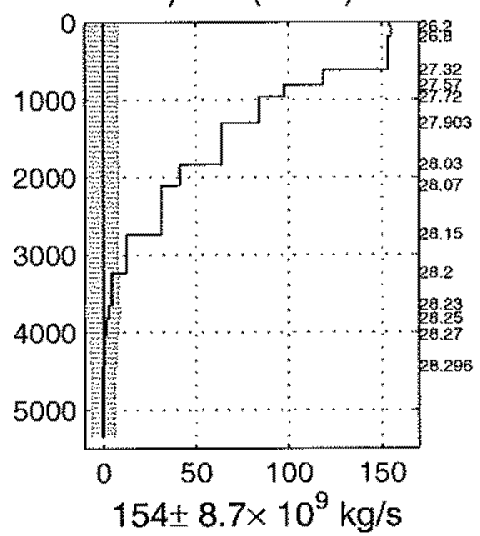

b) A12 (OE)

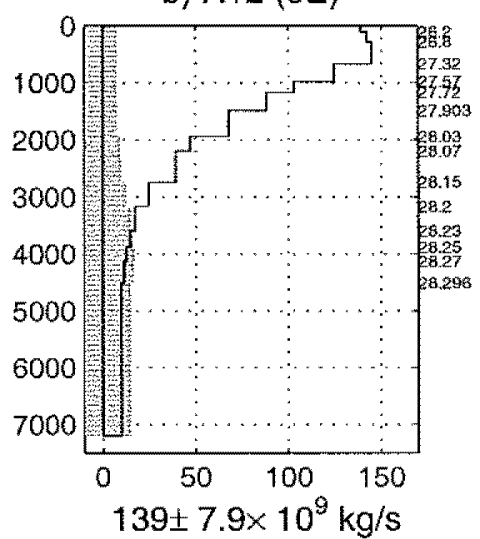

e) P12 (140E)

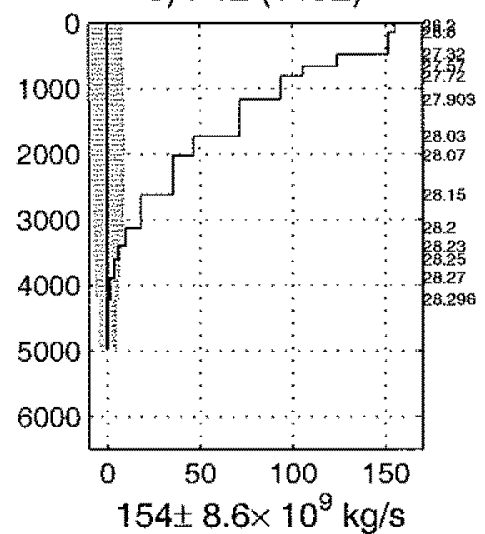

c) $16(30 \mathrm{E})$

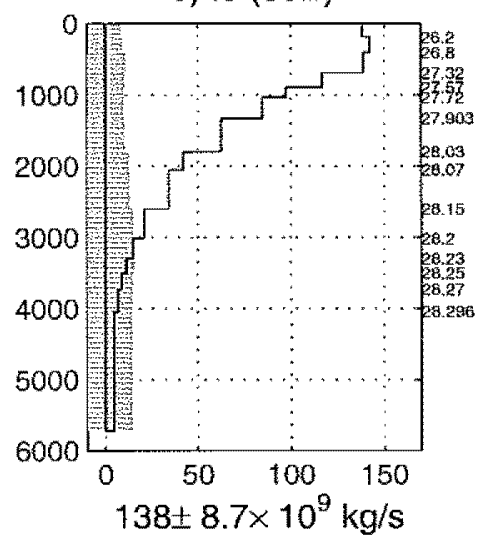

f) $\mathrm{P} 14 \mathrm{~S}(172 \mathrm{E})$

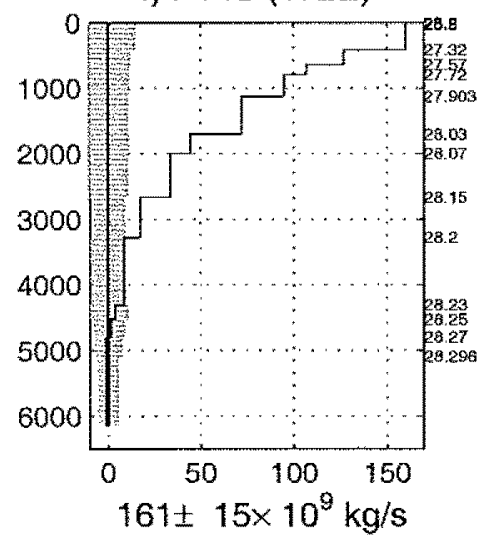

Figure 3-16: Southern Ocean standard circulation. The mass transport across each section is shown $\left(\times 10^{9} \mathrm{~kg} \mathrm{~s}^{-1}\right)$, integrated from the bottom. The shaded area corresponds to the uncertainty (one standard deviation). The net transport is indicated below each graph. The meridional slope of the layers are steep (all layers outcrop), and the depth on the graph indicates the cumulated average thickness of the layers. 
dianeutral diffusivities are uncertain. In the Weddell sea (Fig.3-17a), there is a weak indication of a water mass transfer from dense water to light water of about $6 \pm 7 \mathrm{~Sv}$ near the surface (Hereafter I label such a transfer "upwelling" for convenience, but bearing in mind that the water may as well be transferred horizontally by warming at the outcrop.) Similar upwellings are observed in the "Agulhas" box (Fig.3-17b) and in the Southern Pacific (Fig.3-17e, $13 \pm 5 \mathrm{~Sv}$ ). However, there is dense water production, or downwelling, in the Southern Indian and Southern Australia boxes in this same density range (Fig.317c, d) so that the resulting net diapycnal flux through $\gamma^{n}=27.72$ in the Southern Ocean is not significantly different from zero at $6 \pm 9 \mathrm{~Sv}$.

The surface $\gamma^{n}=27.72$ corresponds to the upper part of NADW in the South Atlantic. Conversion of NADW into AAIW has been suggested in the past (see Schmitz and McCartney, 1993, for a review) and more recently by numerical simulations (Shriver and Hulburt, 1997). However, neither the inverse box models of Macdonald (1998), Sloyan and Rintoul (1999a,b) produced net upwelling across that interface. Here, there an upwelling is found, although not significant.

In deeper layers, deep and bottom water formation is inferred in the Weddell Sea with $-14 \pm 11 \mathrm{~Sv}$ crossing $\gamma^{n}=28.15$. In comparison, Rintoul (1991) estimated AABW production at $9 \mathrm{~Sv}$ based on earlier data. Deep transfers are very uncertain in all other boxes. However, because the uncertainties are correlated, the total southern deep water formation (including entrainment) across $\gamma^{n}=28.15$ is significant, at $-20 \pm 6 \mathrm{~Sv}$. While Macdonald (1998) did not quantify the bottom water formation rate, Sloyan and Rintoul (1999a) obtained a comparable value across $\gamma^{n}=28.20$. However, their water mass transfer just above-across $\gamma^{n}=28.0$, was much larger $(50 \mathrm{~Sv})$. Such a large deep water production would imply a much larger deep inflow into the Pacific and Indian basins than the one that is permitted by this model, as discussed in Sections 3.1 and 3.2.

Existing estimates of bottom water formation in the Southern Ocean bear large uncertainties. The high Sloyan and Rintoul estimate may be related to the COADS heat flux forcing they adopted, and which is very uncertain in the Southern Ocean. Other water mass formation estimates are available from tracers and CFC measurements [e.g., Jacobs et al. (1985), Orsi et al. (1999)], but those depend upon uncertain assumptions about the 
entrainment rates.

Orsi et al. (1999) estimated the total Southern Ocean production of water below $\gamma^{n}=$ 28.27 at $9 \mathrm{~Sv}$ (providing no error bars). But because $\gamma^{n}=28.27$ intersects the bottom south of the northern boundaries of my boxes, the net integrated dianeutral transfer across $\gamma^{n}=28.27$ is null in my model.

In conclusion, my estimate of the Southern Ocean bottom water formation is in agreement with most previous estimates-given the large existing uncertainties. However, I do not recover the large dianeutral fluxes obtained by Sloyan and Rintoul (1999a,b) which would be incompatible with my circulation at lower latitudes. Dianeutral diffusivities in the Southem Ocean are not resolved because of the weak weights on conservation equations, other than mass.

d) S. Australia
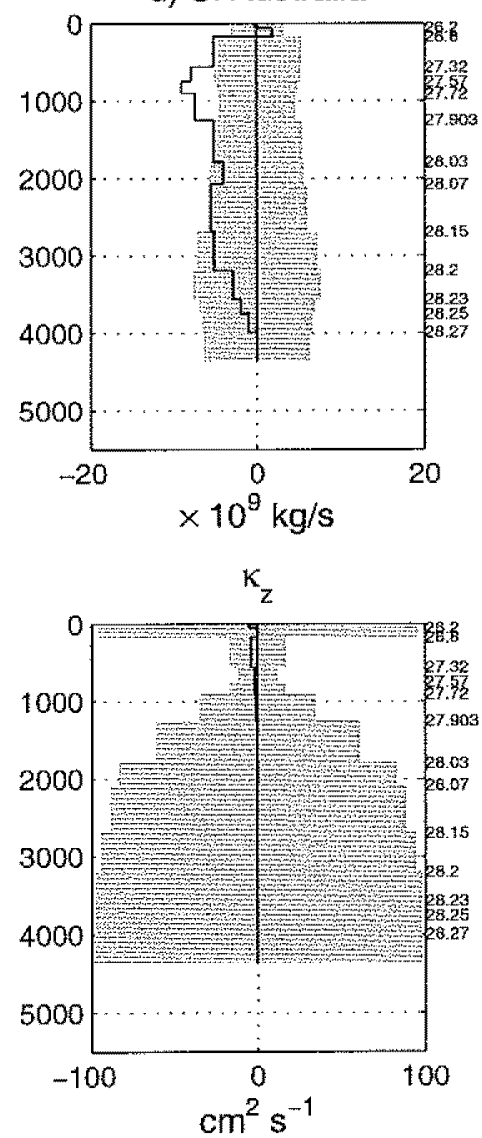

e) S. Pacific
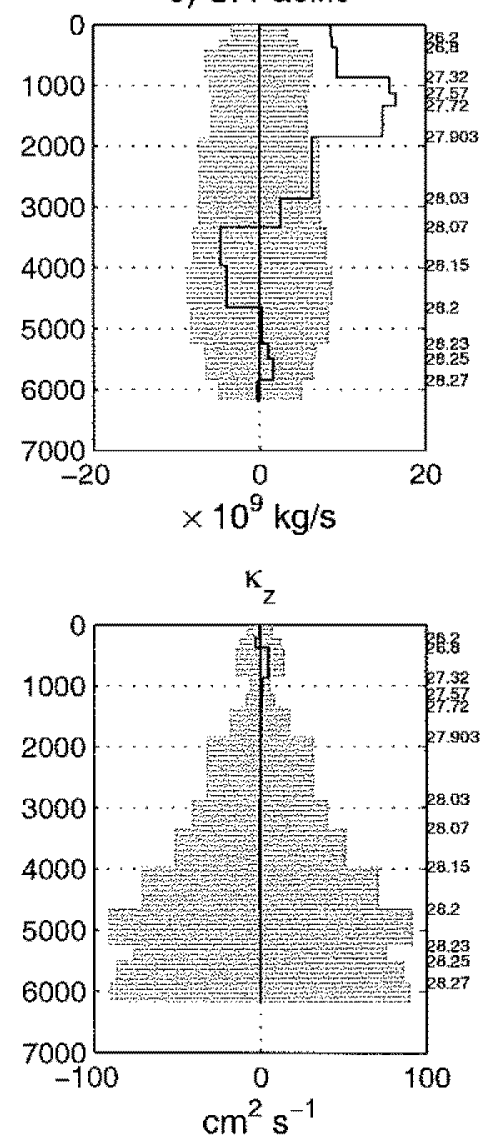

Figure 3-17: (contimued)
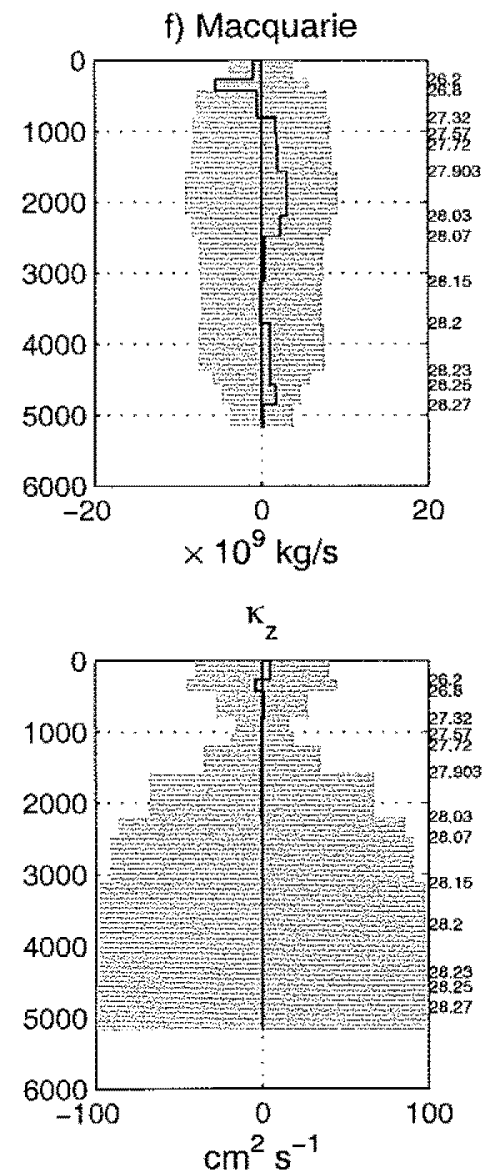
a) Weddell
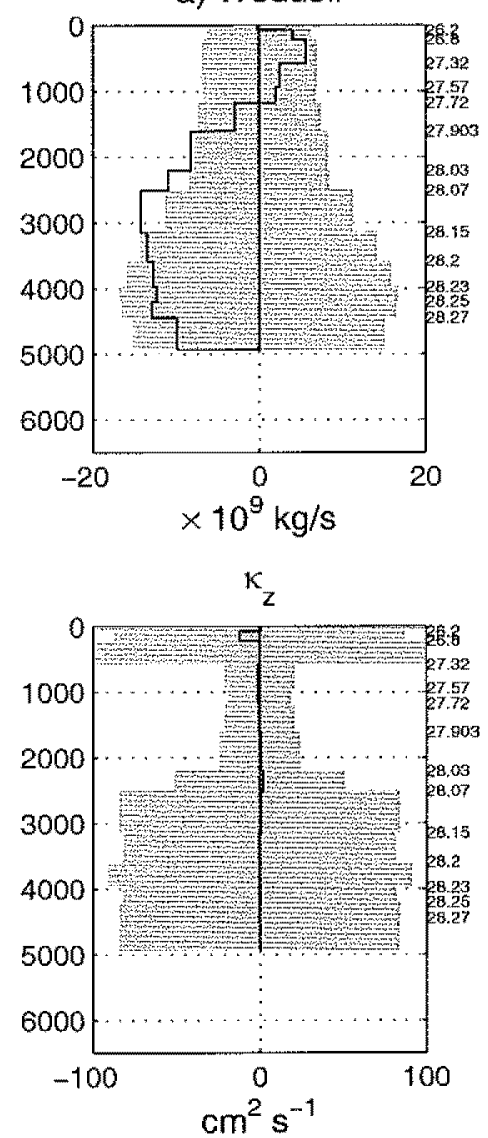

b) S. Agulhas
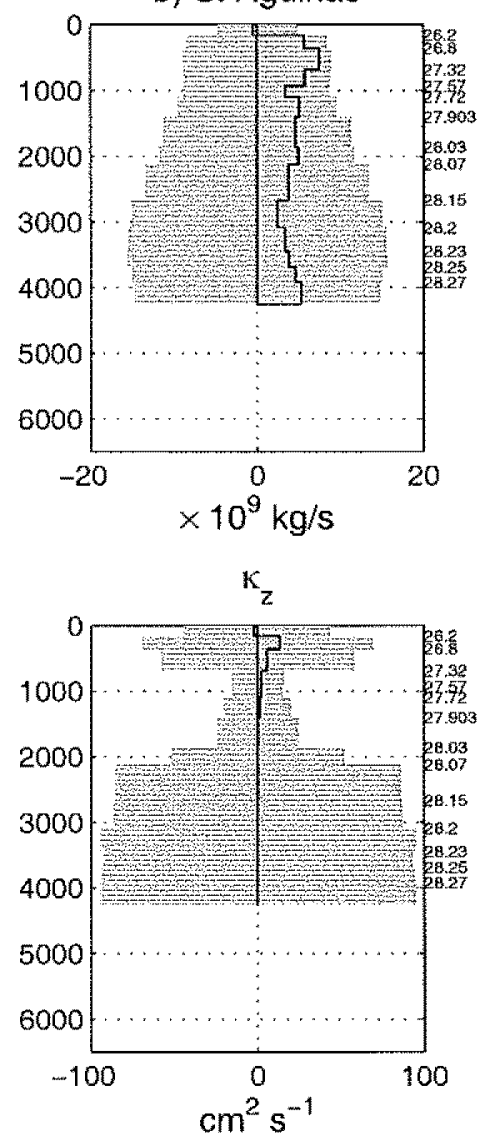

c) S. Indian
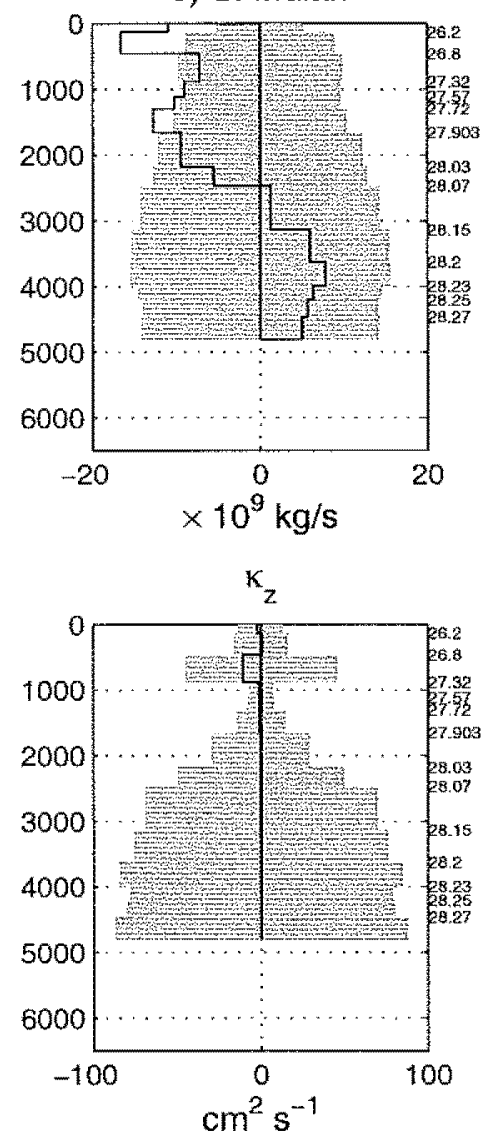

Figure 3-17: Dianeutral transfers (upper) and diffusivities (lower) in the Southern Ocean. The line indicates the transfer or diffusivity at the bottom of the layer. Again, layer depth is just an average. 


\subsubsection{Southern Ocean circulation summary}

The zonal circulation in the Southern Ocean is dominated by the Antarctic Circumpolar Current, with a (constrained) flux of $140 \pm 6 \mathrm{~Sv}$ at Drake Passage. The analysis of dianeutral fluxes gives estimates for the deep and bottom water transformations that are consistent with my global circulation estimate. While the conversion of NADW into AAIW is rather uncertain with $6 \pm 9 \mathrm{~Sv}, 20 \pm 6 \mathrm{~Sv}$ of lower NADW is transformed into bottom water. According to Levitus (1988), northward Ekman transports in the range 15-25 Sv are found around latitude circles in the Southern Ocean, within the range my $6 \pm 9 \mathrm{~Sv}$ water mass conversion estimate. But it is not clear how the Ekman transport, the nonzonality of isopycnal outcrops and other oceanic processes interact to produce the water mass conversion. 


\subsection{Global circulation summary}

In a very idealized summary of the integrated deep water fluxes, my best estimate of the average overturning circulation for the period 1985-1996 can be described as follows. A volume of $16 \pm 2 \mathrm{~Sv}$ of North Atlantic Deep Water was being produced in the northern North Atlantic and moved southward. During its transit through the Atlantic Ocean, Antarctic Bottom Water and Antarctic Intermediate Waters were entrained, increasing the NADW transport to $23 \pm 3 \mathrm{~Sv}$ as it exited the South Atlantic at $30^{\circ} \mathrm{S}$. In the Southern Ocean, a total of $22 \pm 8 \mathrm{~Sv}$ of AABW was formed from lower Circumpolar Deep Water-which corresponds approximately to the lower NADW density range. Bottom water inflows (NADW+AABW mixture) to the Atlantic, Indian and Pacific Oceans are respectively $6 \pm 1.3 \mathrm{~Sv}, 11 \pm 4 \mathrm{~Sv}$ and $8 \pm 2 \mathrm{~Sv}$. In the Indian and Pacific Oceans, most of this water returns southward at deep and intermediate levels.

Due to enhanced variability, the circulation in the upper layers is more uncertain. A northward flow of $18 \pm 3 \mathrm{~Sv}$ of thermocline water from the South Atlantic Ocean balances the NADW southward flow; and the strength of Pacific-Indonesian-Throughflow is estimated at $15 \pm 5 \mathrm{~Sv}$, which is in the higher range of previous estimates.

Generally speaking, the circulation in the Atlantic Ocean is remarkably close to estimates obtained from previous data, suggesting little change in integrated mass transports over the past 30 years. (However, some of the changes or non-changes may be modeldependent, and a more stringent test would be to produce an estimate of the circulation from earlier data using the same treatment and model as used here.) The deep inflow into the Indian Ocean is consistent with previous studies as well. My standard model estimate of the inflow in the South Pacific Ocean is in the lower range of previously published values and depends upon the weight of the PO constraints. Non-conservation of PO yields a higher inflow but produces larger mass residuals in the deep Pacific layers. Closely related, the deep inflow to the North Pacific Ocean is weaker than previously believed, as a consequence of heat and salt conservation in the northern parts of those basins, which 


\begin{tabular}{|l|c|c|c|}
\hline & $W^{*}$ at top $(\mathrm{Sv})$ & $\overline{w^{*}}\left(\times 10^{-4} \mathrm{~cm} \mathrm{~s}^{-1}\right)$ & $\overline{\kappa^{*}}\left(\mathrm{~cm}^{2} \mathrm{~s}^{-1}\right)$ \\
\hline Atlantic bottom & $5 \pm 2$ & $0.6 \pm 0.5$ & $7 \pm 6$ \\
\hline Indian bottom & $4 \pm 2$ & $0.5 \pm 0.5$ & $12 \pm 16$ \\
\hline Pacific bottom & $9 \pm 2$ & $0.4 \pm 0.2$ & $14 \pm 4$ \\
\hline Southern bottom & $-20 \pm 6$ & $-0.4 \pm 0.3$ & - \\
\hline \hline Atlantic deep & $8 \pm 3$ & $0.2 \pm 0.05$ & $3 \pm 2$ \\
\hline Indian deep & $9 \pm 4$ & $0.26 \pm 0.15$ & $3.5 \pm 2$ \\
\hline Pacific deep & $7 \pm 3$ & $0.08 \pm 0.06$ & $4.4 \pm 1.3$ \\
\hline Southern deep & $6 \pm 9$ & $0 \pm 0.2$ & - \\
\hline
\end{tabular}

Table 3.17: Basin-averaged dianeutral velocities and diffusivities. The average is calculated on neutral surfaces from $\gamma^{n}=28.1$ to the bottom (generally 3800 dbars to the bottom) for the "bottom layers" and from $\gamma^{n}=27.96$ to $\gamma^{n}=28.07$ for the "deep layers". The second column indicates the total transport in the basin through the top interface, while the third and forth columns indicate the average dianeutral velocities and upwelling.

were not generally considered in previous models.

Associated with the circulation, significant dianeutral exchanges were found. Table 3.17 summarizes the dianeutral exchanges basin-wise in the deep and bottom layers. Dianeutral velocities are in the range $0.1-0.6 \times 10^{-4} \mathrm{~cm} \mathrm{~s}^{-1}$ while the diffusivities are in the range $3-4 \mathrm{~cm}^{2} \mathrm{~s}^{-1}$ in the deep layers, and larger near the bottom. (Diffusivities were not resolved in the Southern Ocean, where isopycnals outcrop.) Bottom water formation in the Southern Ocean could be diagnosed consistently with the global circulation, implying a dianeutral velocity of $-0.4 \pm 0.3 \times 10^{-4} \mathrm{~cm} \mathrm{~s}^{-1}$. The global average dianeutral velocity over the Atlantic, Pacific and Indian Oceans North of $30^{\circ} \mathrm{S}$ is of $\overline{w^{*}}=0.56 \pm 0.36 \times 10^{-4} \mathrm{~cm} \mathrm{~s}^{-1}$ in the bottom layers and of $\overline{w^{*}}=0.16 \pm 0.04 \times 10^{-4} \mathrm{~cm} \mathrm{~s}^{-1}$ in the deep layers. The average diffusivity is of $\overline{\kappa^{*}}=9 \pm 5 \mathrm{~cm}^{2} \mathrm{~s}^{-1}$ in the bottom layers and of $\overline{\kappa^{*}}=3.4 \pm 1.3 \mathrm{~cm}^{2} \mathrm{~s}^{-1}$ in the deep layers (these averages were not weighted by the area, however).

This simplified circulation scheme is based on integrated mass transports calculations, and, as we will see in the next Chapter, does not always directly determine the actual property transports. 


\section{Chapter 4}

\section{Global Property Fluxes and}

\section{Divergences}

In this chapter, fluxes of freshwater, heat, nutrients and oxygen as diagnosed from the circulation scheme are analyzed. The analysis starts with the freshwater flux divergences (Section 4.1); energy flux estimates are then discussed (Section 4.2) followed by silica (4.3.1), nitrate and phosphate (Section 4.3.2) and oxygen (Section 4.4) transports. Transports and residuals of nutrients and oxygen are discussed in relation to biological activity. In particular, the residuals allow an estimate of the export production. Net property transports across sections are listed in Appendix E.

\subsection{Freshwater flux divergences}

Freshwater divergences are calculated as part of the solution (Eq.1.8 and 1.9, page 20) through the anomaly formulation, subject to the assumptions about the errors described in Chapter 2. Inferred precipitation plus runoff minus evaporation (P-E) in the standard solution are given for the different oceans and compared with other authors in Tables 4.1 to 4.3 .

In the Indian Ocean (Table 4.1), freshwater divergences are indistinguishable from zero in the North Indian Ocean sector, north of $8^{\circ} \mathrm{S}$ (I2-I10, $0.1 \pm 0.3 \mathrm{~Sv}$ net precipitation). South of $8^{\circ} \mathrm{S}$, the solution shows net evaporation in the tropical $\left(20^{\circ} \mathrm{S}-8^{\circ} \mathrm{S}\right)$ and subtropical 


\begin{tabular}{|l|c|c|c|}
\hline & Indian & \\
\hline Between & $32^{\circ} \mathrm{S}$ & $20^{\circ} \mathrm{S}$ & $8^{\circ} \mathrm{S}$ \\
and & $20^{\circ} \mathrm{S}$ & $8^{\circ} \mathrm{S}$ & Coast \\
\hline Present study & $-0.35 \pm 0.25$ & $-0.3 \pm 0.2$ & $+0.1 \pm 0.3$ \\
\hline Jourdan et al. (1997) & -0.3 & -0.5 & \\
\hline Wijffels et al. (1992) & -0.2 & -0.45 & \\
\hline Macdonald (1996) & -0.4 & & \\
\hline Oberhuber (1988) & $\longleftarrow-0.4 \longrightarrow$ & +0.25 \\
\hline
\end{tabular}

Table 4.1: Precipitation (+) minus evaporation (-) estimates for the different oceanic regions bounded by the model sections in the Indian Ocean. Units are in Sv.

\begin{tabular}{|l|l|l|c|l|}
\hline & \multicolumn{5}{|l|}{ Pacific } & \multicolumn{3}{l|}{} \\
\hline Between & $30^{\circ} \mathrm{S}$ & $17^{\circ} \mathrm{S}$ & $24^{\circ} \mathrm{N}$ & $47^{\circ} \mathrm{N}$ \\
and & $17^{\circ} \mathrm{S}$ & $24^{\circ} \mathrm{N}$ & $47^{\circ} \mathrm{N}$ & Coast \\
\hline Present study & $-0.3 \pm 0.26$ & $+0.1 \pm 0.3$ & $+0.14 \pm 0.2$ & $+0.1 \pm 0.7$ \\
\hline Jourdan et al. (1997) & -0.5 & -1.2 & 0 & +0.3 \\
\hline Wijffels et al. (1992) & -0.2 & +0.3 & 0 & \\
\hline Macdonald (1996) & & & 0 & \\
\hline Tsimplis et al. (1998) & -0.4 & & & \\
\hline RMC89 & & & 0 & \\
\hline
\end{tabular}

Table 4.2: Same as Table 4.1 but for the Pacific Ocean. RMC89=Roemmich and McCallistair (1989)

boxes $\left(32^{\circ} \mathrm{S}-20^{\circ} \mathrm{S}\right)$ (a minus sign denotes net evaporation). These two latter estimates are consistent with the estimates from satellite radiometric data of Jourdan et al. (1997), (Table 4.1, error bars are not given for these latitude bands, but they probably do not exceed 100\%). Wijffels et al. (1992), Macdonald (1995) and Oberhuber (1988) report estimates in the same range. (Note that the Wijffels et al.(1992) is essentially an integration of the Baumgartner and Reichel (1975) estimate.) Oberhuber's (1988) values have a greater contrast north and south of $10^{\circ} \mathrm{S}$, but are within our error bars. Overall, the Indian Ocean is evaporative, with $-0.6 \pm 0.4 \mathrm{~Sv}$ net air-sea exchange. As for all property fluxes, the model's large Pacific-Indonesian Throughflow may affect substantially the estimated freshwater divergences over the Indian Ocean.

In the South Pacific (Table 4.2), net evaporation characterizes the subtropical region, in agreement with the estimates of Jourdan et al. (1997) and the inversion of Tsimplis et al. (1998). It encompasses the Wijffels et al. (1992) estimate within uncertainties. North 


\begin{tabular}{|c|c|c|c|c|c|c|c|}
\hline & \multicolumn{7}{|l|}{ Atlantic } \\
\hline From & $48^{\circ} \mathrm{N}$ & $24^{\circ} \mathrm{N}$ & $7.5^{\circ} \mathrm{N}$ & $4.5^{\circ} \mathrm{S}$ & $11^{\circ} \mathrm{S}$ & $19^{\circ} \mathrm{S}$ & $30^{\circ} \mathrm{S}$ \\
\hline to & $24^{\circ} \mathrm{N}$ & $7.5^{\circ} \mathrm{N}$ & $4.5^{\circ} \mathrm{S}$ & $11^{\circ} \mathrm{S}$ & $19^{\circ} \mathrm{S}$ & $30^{\circ} \mathrm{S}$ & $45^{\circ} \mathrm{S}$ \\
\hline Present & $0 \pm 0.15$ & $-0.1 \pm 0.3$ & $0.1 \pm 0.4$ & $0.2 \pm 0.5$ & $-0.3 \pm 0.3$ & $-0.36 \pm 0.2$ & $0.45 \pm 0.2$ \\
\hline JPG1997 & -0.2 & -0.4 & +0.2 & -0.15 & -0.25 & -0.25 & \\
\hline SBD1989 & -0.3 & -0.2 & & & & & \\
\hline WSB1992 & -0.3 & -0.2 & +0.2 & -0.1 & -0.2 & -0.2 & \\
\hline M1995 & -0.1 & 0 & $\longleftarrow+0$ & $15 \longrightarrow$ & -0.4 & & \\
\hline
\end{tabular}

Table 4.3: Same as Table 4.1but for the Atlantic Ocean (JPG1997= Jourdan et al. (1997); SBD1989=Schmitt et al. (1989); WSB1992= Wijffels et al. (1992); M1995=Macdonald (1995))

of $17^{\circ} \mathrm{S}$, freshwater divergences are more uncertain. Our result in the Central Pacific is incompatible with the large evaporation found by Jourdan et al. (1997)-although the uncertainty on the later is probably at least $100 \%$. In contrast, the model estimate for the Pacific Ocean north of $17^{\circ} \mathrm{S}$ is for net precipitation: a total of $0.3 \pm 0.3 \mathrm{~Sv}$. The net freshwater budget over the Pacific Ocean north of $32^{\circ} \mathrm{S}$ is balanced within the uncertainties, $0 \pm 0.4 \mathrm{~Sv}$.

The North Atlantic Ocean freshwater flux divergences are weak and uncertain (Table 4.3). Our estimates agree with Macdonald's (1995) within one standard deviation. The evaporation-precipitation magnitudes reported by Jourdan et al. (1997), Schmitt et al. (1989) and Wijffels et al. (1992) are larger, although within two standard deviations of our estimate. In the South Atlantic, freshwater flux divergence estimates are larger-but still encompass zero within an uncertainty of two standard deviations. Both size and magnitude are consistent with previous estimate. Over the central Atlantic between $24^{\circ} \mathrm{N}$ and $19^{\circ} \mathrm{S}$, the model estimates a net evaporation of $-0.1 \pm 0.2 \mathrm{~Sv}$, in agreement with the Wijffels estimate $(-0.2 \mathrm{~Sv})$, but smaller than the radiometric measurements $(-0.55 \mathrm{~Sv})$. Overall, the model's Atlantic freshwater flux divergence is generally uncertain, but predicts net evaporation of $-0.5 \pm 0.3 \mathrm{~Sv}$ between $30^{\circ} \mathrm{S}$ and $47^{\circ} \mathrm{N}$.

In the Southern Oceans, freshwater flux divergences (not shown) are even more uncertain due to the low weight of the salinity equations there. The net air-sea flux estimate south of $30^{\circ} \mathrm{S}$ is of precipitation at $0.8 \pm 0.9 \mathrm{~Sv}$, which is approximately in balance with the net global evaporation between $30^{\circ} \mathrm{S}$ and $47^{\circ} \mathrm{N},-1.2 \pm 0.5 \mathrm{~Sv}$. 
Except in few places, freshwater divergences are relatively uncertain. Given the large error bars, there is overall agreement with previous studies. Figure 4-1 summarizes the freshwater divergences (numbers in the boxes). For completeness, those divergences were integrated from arbitrary references to compute the so-called "freshwater transports" across each section. But any non-divergent field could be added to those transports, and only the divergence is of physical interest. Nevertheless, for comparison the integration of the freshwater divergences was done as follows: 1) The "freshwater flux" through the Bering Strait was taken equal to $0.79 \pm 0 \mathrm{~Sv}$ at $47^{\circ} \mathrm{N}$ in the Pacific Ocean and to $-0.95 \pm 0 \mathrm{~Sv}$ at $47^{\circ} \mathrm{N}$ in the Atlantic Ocean, that is, following the Wijffels et al. (1992) calculation. Through the Pacific-Indonesian Throughflow and the Drake Passage, the freshwater flux was set to $0 \pm 0 \mathrm{~Sv}$ (the uncertainties are taken to zero, because of the arbitrariness of adding a non-divergent field). 2) Freshwater divergences were integrated, starting, in the Atlantic Ocean, at $30^{\circ} \mathrm{S}$; in the Pacific Ocean, at $47^{\circ} \mathrm{N}$; and in the Indian Ocean, at the northern boundary. Those starting points were chosen to minimize the uncertainties on the freshwater transports. In the Southern Ocean, freshwater transports were calculated from the southern Atlantic box. In principle, the calculation in the Pacific Ocean is a continuation of the integration from the Southern Ocean. But because of the large uncertainty in the Southern Ocean, it was preferred to integrate the Pacific Ocean freshwater divergences from the North Pacific, resulting in a small shift in the freshwater flux at $30^{\circ} \mathrm{S}$ in the Pacific Ocean. 


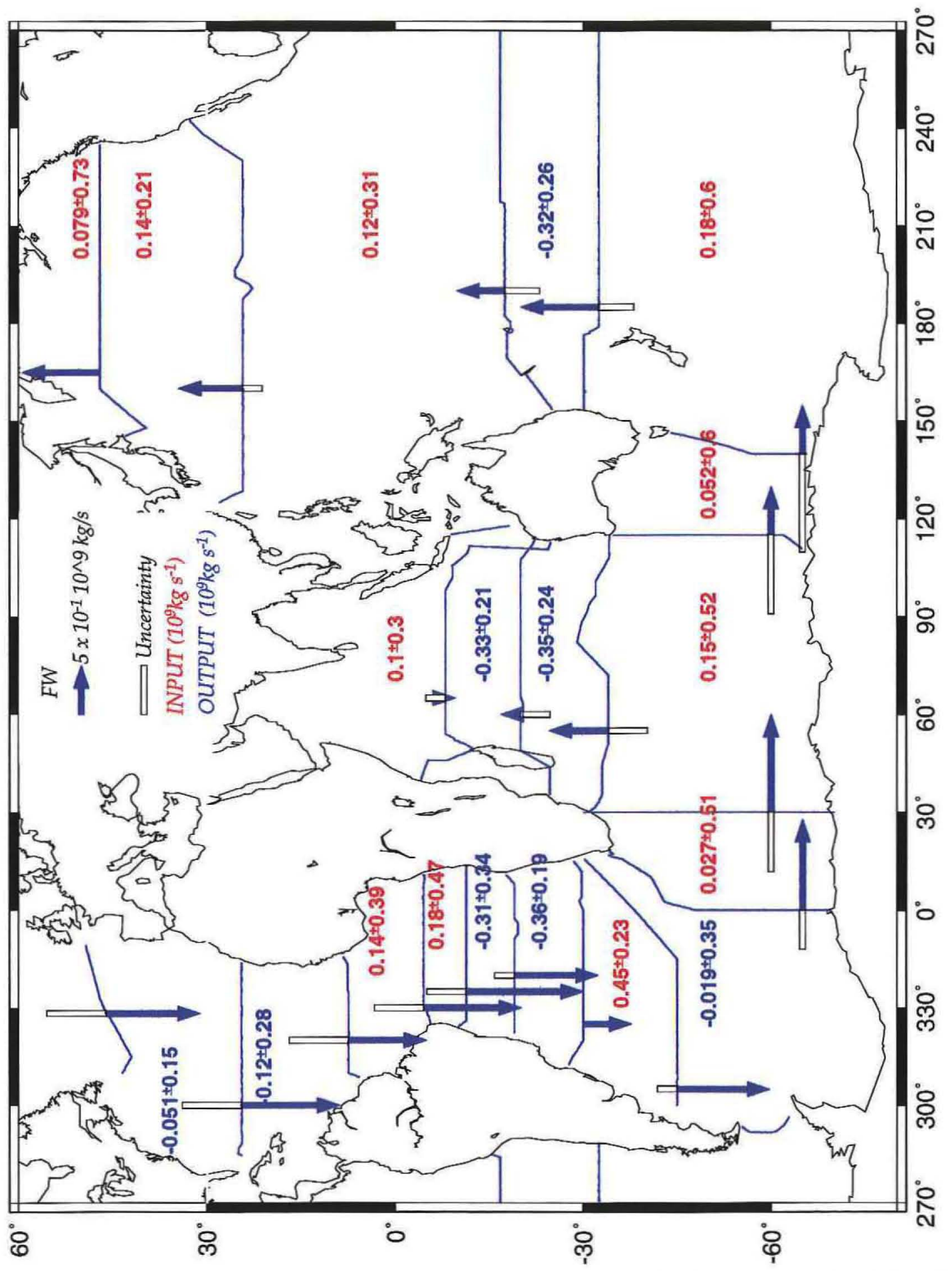

Figure 4-1: Freshwater divergences and transports. While the divergences (positive for precipitation/negative for evaporation) are diagnosed from the model, net transports are integrated arbitrarily and rely upon stringent assumptions (see text). 


\subsection{Heat transports}

The oceanic energy flux substantially regulates the Earth's climate. Simulations suggest that changes in the thermohaline circulation strength have a large impact on atmospheric temperature gradients which control climate (e.g., Manabe and Stouffer, 1988; Marotzke, 1996 ). Existing estimates from surface observations are highly uncertain (Gleckler and Weare, 1997; Josey et al., 1999). For instance, Josey et al. (1999) found a positive bias of 10 to $15 \mathrm{Wm}^{-2}$ over large oceanic regions. Integrated over the Indian Ocean, the bias would imply an overestimate of about 0.4 Petawatts $\left(1 \mathrm{PW}=10^{15} \mathrm{~W}\right)$, that is, $100 \%$ of the Indian Ocean heat gain as estimated, for instance, by Hastenrath and Greishar (1993). But even after correction, Josey et al. (1999) found a global heat imbalance of $30 \mathrm{~W} \mathrm{~m}^{-2}$, suggesting large residual uncertainties.

Analyses from numerical weather prediction centers provide oceanic surface fluxes that are often used as a boundary condition to force ocean models. But meteorological models do not provide uncertainties. Using a heuristic approach, Trenberth and Solomon (1994)

proposed an uncertainty of $30 \mathrm{~W} \mathrm{~m}^{-2}$ over the oceans, based on the European Centre for Medium Range Weather Forecasts (ECMWF) outputs. The consequent uncertainties in global oceanic heat transports were calculated as 0.3 to 0.5 PW. From a comparison between ECMWF, National Meteorological Center (NMC) and net radiative fluxes from satellite data, Keith (1995) did a similar calculation and obtained larger uncertainties around $0.6 \mathrm{PW}$ for the meridional oceanic energy transport at most latitudes. Recently, Garnier et al. (1999) studied the new ECMWF 15-year re-analysis and found a remaining global heat imbalance of $8 \mathrm{~W} \mathrm{~m}^{-2}$, implying a systematic error over the ocean.

Arguably, the most accurate energy flux estimations come from circulation estimates based on hydrographic sections. Reported flux uncertainties are generally in the range \pm 0.3 PW (e.g., Macdonald, 1998; Holfort and Siedler, 1999). The present estimate of the global heat transports indicates uncertainties that depend on latitude, ranging between $\pm 0.1 \mathrm{PW}$ and $\pm 0.5 \mathrm{PW}$. 


\subsubsection{Global energy transports and heat transfers}

Energy transports are given across each section. However, those are expressed in units of Petawatts referred to the Celsius scale for unit consistency with most of the literature. Warren (1999) argues, convincingly, that the terminology "heat flux" is inappropriate in this context (see also, Bohren and Albrecht, 1998, pp.24-28) and one should refer to the "energy flux", although it does not include the kinetic energy-which is a small contribution in this case.

Because the model privileges mass conservation in the deep layers at the expense of the top-to-bottom mass conservation (i.e., Fig. 2-12, page 64), the net flux through individual sections is not strictly mass-conservative in semi-closed basins. This noise increases towards low latitudes. But in contrast with net fluxes, estimated heat anomaly residuals are mass-conserving by definition, and provide a more reliable diagnostic of atmospheric heat exchanges. The energy flux divergence in a layer that is in contact with the sea surface may be written

$$
\mathrm{D}_{0}=\oint \rho v \mathrm{C}_{\mathrm{p}} \theta \mathrm{ds}+\kappa \int_{\text {lower interface }} \frac{\partial \rho \mathrm{C}_{\mathrm{p}} \theta}{\partial z} \mathrm{ds}+n_{\theta},
$$

where the first integral is made over the lower and side boundaries of the layer and the second integral is made over the lower interface. $\rho$ is in situ density; $v$ is the velocity across the boundary; $\mathrm{C}_{\mathrm{p}}$ is heat capacity; $\theta$ is potential temperature; $\kappa$ is dianeutral diffusion and $n_{\theta}$ is the noise in the heat divergence. The contribution from spurious mass divergences is removed from $n_{\theta}$, defining the heat anomaly residuals (see also Section 1.2.4).

$$
\Delta_{\theta}=\mathrm{D}_{\theta}-\bar{\theta} \oint \rho v \mathrm{ds}
$$

Where $\vec{\theta}$ is the average potential temperature in the layer. There is no assumption that the noise cancels out (i.e., $n_{\theta} \simeq \bar{\theta} \oint \rho v$ ds) in this diagnostic, and the uncertainty of $\Delta_{\theta}$ is computed explicitly from the solution. In contrast with $\Delta_{\theta}, \mathrm{D}_{\theta}$ does not depend upon the temperature scale because the mass divergence has been removed. Diagnosing $\Delta_{\theta}$ instead of $\mathrm{D}_{\theta}$ is an arbitrary choice because the uncertainty in $\mathrm{D}_{\theta}$ already takes into account the spurious mass divergence. However, several simple examples (e.g., Appendix B) suggest that $\Delta_{\theta}$ is closer to the true energy divergence in the presence of spurious mass noise. 
The uncertainty on $\Delta_{\theta}$ and on the divergences of all tracers is computed using the full covariance matrix of the solution. Because of the existing correlations between errors the uncertainties of divergences are occasionally much lower than suggested by a visual differentiation of the fluxes.

The formal uncertainties of energy transports and residuals include the part due to oceanic variability in the mass flux. However, the uncertainty due to temporal variations in the temperature field is not taken into account. Holfort and Siedler (1999) gave a rough estimate of $\pm 0.2 \mathrm{PW}$ for this contribution in the South Atlantic. The authors do not provide much detail on the method used to obtain this number, and a more accurate estimate would be useful, from XBT data or GCM simulations for instance.

Figure 4-2 shows the total energy fluxes across latitudes or longitude lines (arrows, the white boxes giving the uncertainty). The energy flux is northward everywhere in the Atlantic Ocean.

Heat residuals in each region were computed as the sum of anomaly residuals of each . layer of the model (outcropping and interior layers). [Because of the large differences in the average temperature in surface and deep layers, heat anomalies must be defined using the average temperature of individual layers rather than the top-to-bottom value (e.g., see the discussion on silica anomalies Section 1.2.4, page 24).]

There is a net and significant cooling in the North Atlantic and North Pacific between $24^{\circ} \mathrm{N}$ and $48^{\circ} \mathrm{N}$ (respectively $-0.5 \pm 0.1 \mathrm{PW}$ and $-0.6 \pm 0.2 \mathrm{PW}$, Figure 4-2). For completeness, this figure was also produced with the divergences given in units of $\mathrm{W} \mathrm{m}^{-2}$ (Figure 4-3). At low latitudes, warming is observed in both Atlantic and Pacific. In the Atlantic, heat residuals in individual basins are uncertain but coherent, so that the total warming between $30^{\circ} \mathrm{S}$ and $24^{\circ} \mathrm{N}$ in the Atlantic is significant, at $0.8 \pm 0.2 \mathrm{PW}$. In the Indian Ocean, residuals are noisy everywhere. The Indian Ocean heat budget is tightly related to the strength of the Pacific-Indian throughflow, as discussed below. In the central Pacific Ocean, there is a net heat gain of $1.75 \pm 0.4 \mathrm{PW}$.

In the Southern Ocean there is a marginal indication of a net cooling in the Indian sector and south of Australia. But in the southern Pacific, there is a significant heating $(0.45 \pm 0.2 \mathrm{PW})$ in Layer 3 , which outcrops south of $50^{\circ} \mathrm{S}$ (south of Tasmania). 


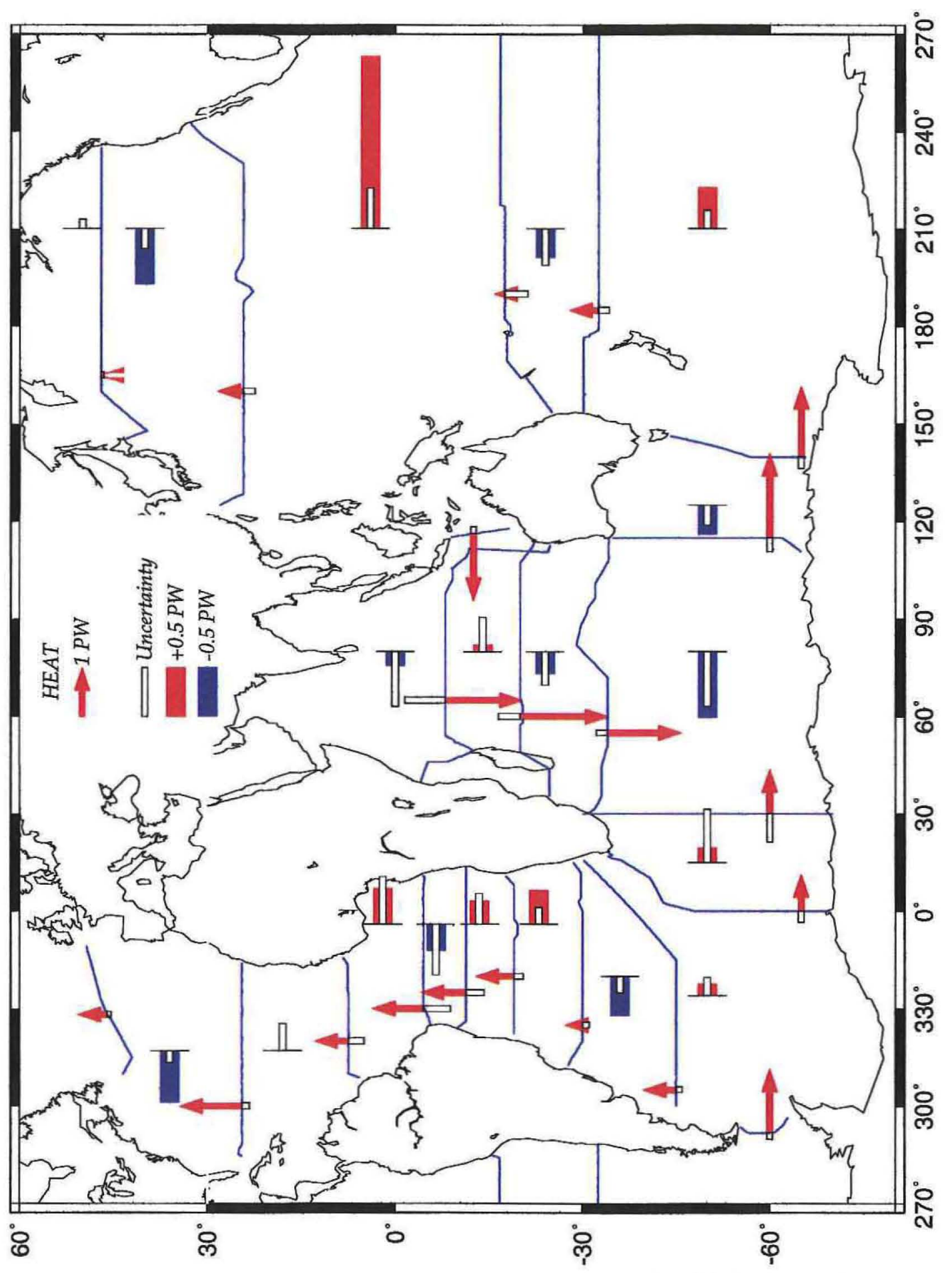

Figure 4-2: Global temperature transports and heat residuals. Each arrow denotes the temperature transports between continents. The white boxes correspond to the uncertainties (one standard deviation). Between sections, heat residual anomalies are indicated by the full boxes. 


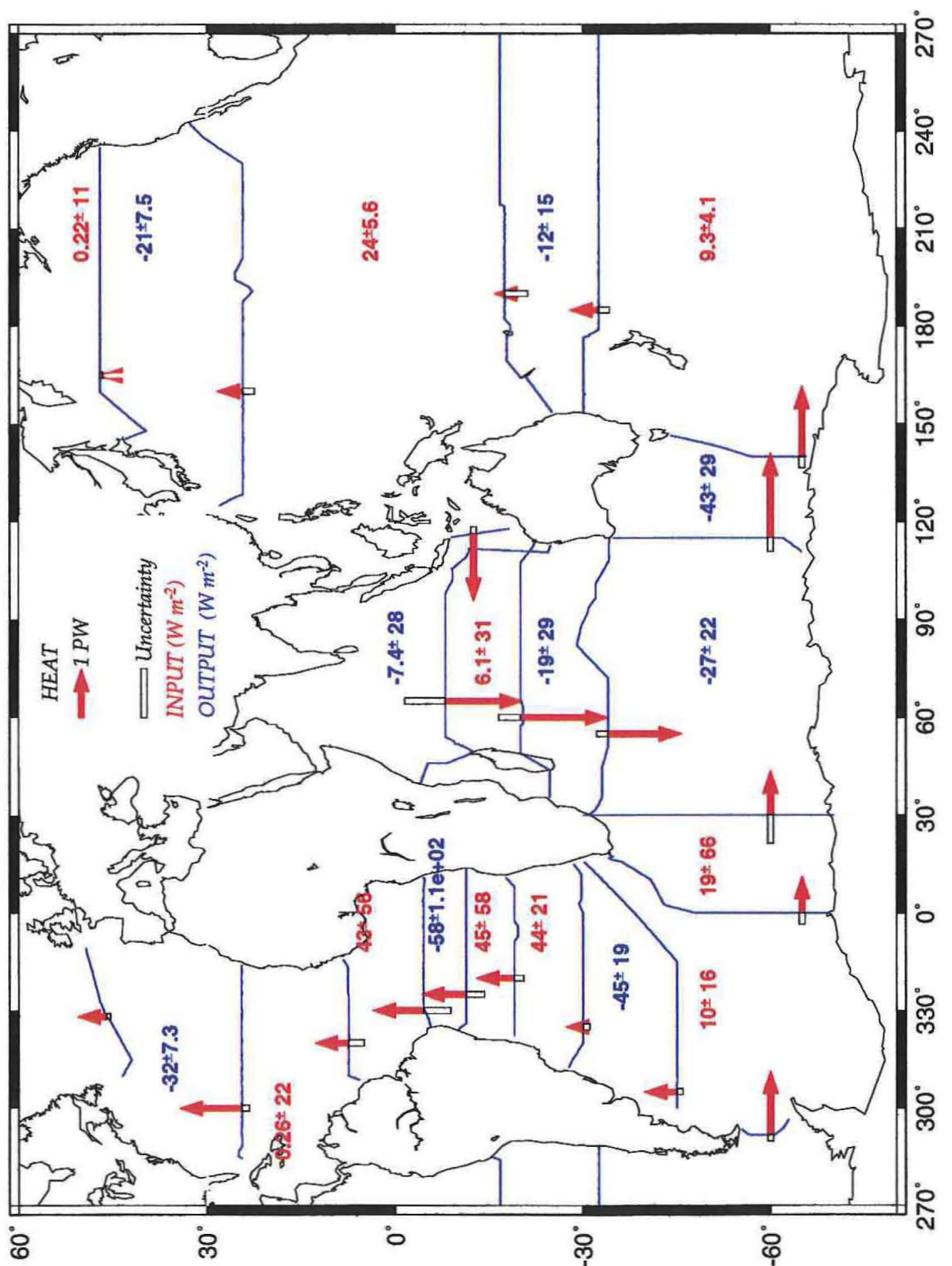

Figure 4-3: Same as Fig. 4-2, but the residuals are expressed in Watts per square meter. 
The map of heat transfers derived from the ECMWF re-analysis (Garnier et al., 1999) bears qualitative similarities to our estimates, with most of the oceanic heating occurring in eastern and equatorial upwelling regions, while most of the oceanic cooling occurs in the western boundary current regions of the northern hemisphere and at high latitudes. In the southern hemisphere, cooling was also indicated in the western boundary currents but the geographic distribution of the currents is such that there is an equivalent heating in the same latitude bands, so that the total heat transfers in each of our boxes are relatively small. (Generally, oceanic heating or cooling seems to be correlated with ocean currents more than it is with latitude.) In particular, the ECMWF heat transfers indicate oceanic heating between $40^{\circ} \mathrm{S}$ and $65^{\circ} \mathrm{S}$, thus consistent with the southern Pacific heating that is calculated here. The Comprehensive Ocean-Atmosphere Data Set (COADS, da Silva et al., 1996) also indicates global heating around $50^{\circ} \mathrm{S}$, but a strong cooling between $43^{\circ} \mathrm{S}$ and $15^{\circ} \mathrm{S}$ as well. (Robbins et al. (personal communication, 1999) suggest that COADS estimates is erroneous in this region because of the lack of data.)

In summary, given the large range of existing energy flux estimates and uncertainties, we find no qualitative incompatibilities with our estimate. We now turn to the quantitative comparison.

\section{Atlantic energy fluxes}

Figure 4-4 compares our estimate of the Atlantic energy fluxes (black stars) with other published estimates. The Trenberth and Solomon (1994) uncertainties were derived heuristically, assuming that spurious heat residuals on land were representative of the energy flux uncertainties everywhere. In this view, they provide a lower bound on the actual uncertainty of the ECMWF model. The Semtner and Chervin estimate corresponds to the "POCM-4B:" integration (Semtner and Chervin, 1992; Stammer et al., 1996; Jayne, 1999).

There are no incompatibilities, within one standard deviation, with the previous hydrographic estimates. The present net energy flux across $7.5^{\circ} \mathrm{N}$ is probably underestimated because of the high variability in the western part of this section (e.g., Johns et al., 1998). Heat residuals, because they are corrected for the spurious mass imbalances that 


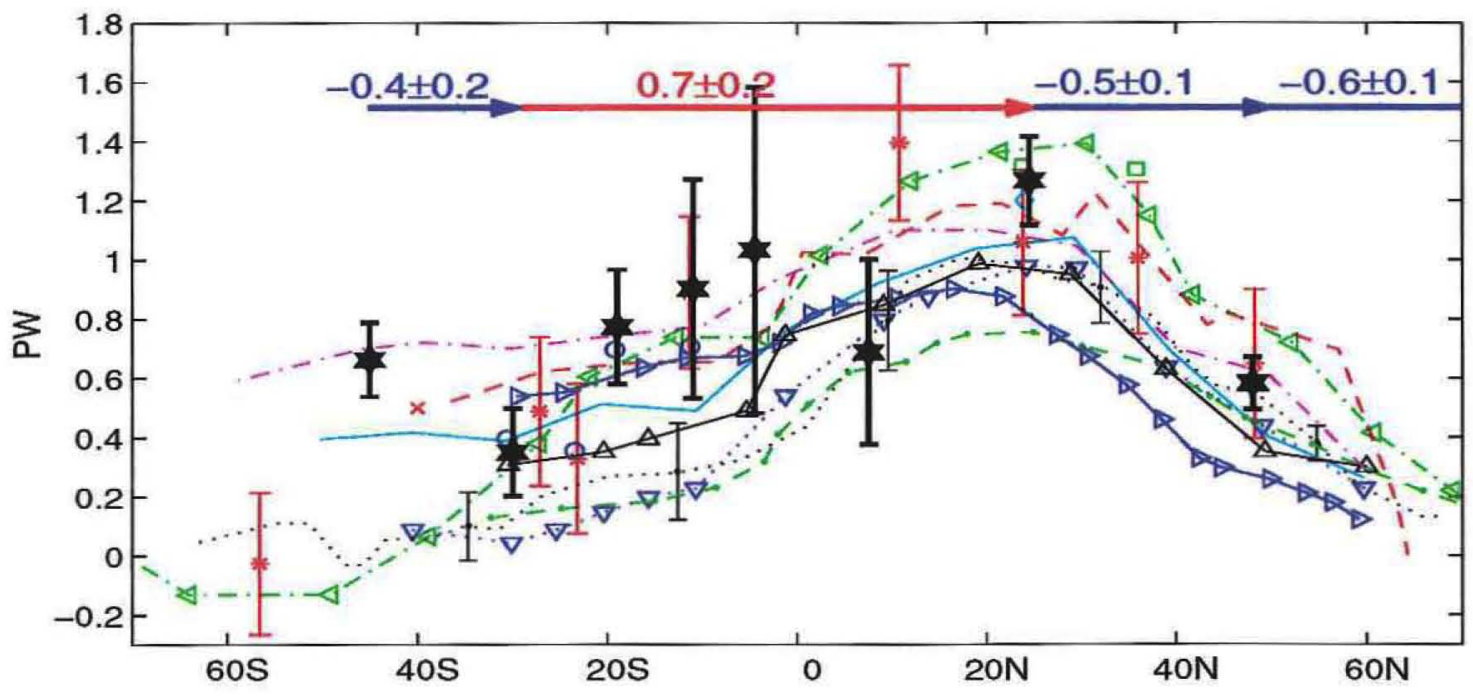

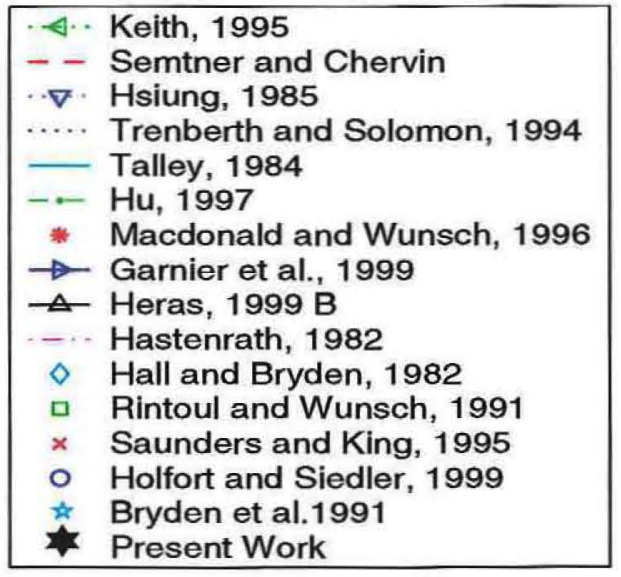

Figure 4-4: Atlantic energy transports (black stars with thick error bars) and residuals between selected latitudes (numbers above the northward-pointing arrows, positive for heating). (The energy flux at $7.5^{\circ} \mathrm{N}$ is probably noisy due to variability in the North Brazil Current region.) Errorbars from Holfort and Siedler (1999) are of $\pm 0.3 \mathrm{PW}$; Rintoul and Wunsch (1991), \pm 0.2 PW; Hall and Bryden (1982), \pm 3 PW; and Saunders and King (1995), $\pm 0.1 \mathrm{PW}$. (Adapted and completed from Macdonald and Wunsch (1996) and Heras and Schlitzer (1999).) 
are present in the surface layers, indicate an uncertain ocean warming between $4.5^{\circ} \mathrm{S}$ and $7.5^{\circ} \mathrm{N}(0.35 \pm 0.5 \mathrm{PW})$.

Heat residuals integrated over large latitude bands are indicated on Figure 4-4 by the numbers on the blue and red arrows. While residuals in individual boxes are uncertain and noisy at low latitudes, the total warming over the central Atlantic $\left(30^{\circ} \mathrm{S}-24^{\circ} \mathrm{N}\right)$ is significant, with $0.7 \pm 0.2 \mathrm{PW}$ warming. Poleward of this latitude band, net cooling is observed, with $-0.3 \pm 0.15 \mathrm{PW}$ in the South Atlantic (A11 to $30^{\circ} \mathrm{S}$ ); $-0.5 \pm 0.1 \mathrm{PW}$ in the North Atlantic $\left(24^{\circ} \mathrm{N}-48^{\circ} \mathrm{N}\right)$ and $-0.6 \pm 0.1 \mathrm{PW}$ in the northern North Atlantic (north of $\left.48^{\circ} \mathrm{N}\right)$.

Except for the Keith (1995) estimate in the North Atlantic, most other estimates from GCM, COADS and National Weather Prediction centers are lower than ours in the Atlantic Ocean. In the North Atlantic, there is a fair agreement about the heat loss between $24^{\circ} \mathrm{N}$ and $48^{\circ} \mathrm{N}$ (except for Hu, 1997 and Semtner and Chervin). The warming inferred in the central Atlantic is compatible with most estimates, except $\mathrm{Hu}$ (1997) and Hastenrath (1982), which have a lower heat gain. Between A11 (plotted at $45^{\circ} \mathrm{S}$ on Fig. 4-4) and $30^{\circ} \mathrm{S}$, no climatology indicates the same cooling that I found. But because section A11 is not entirely zonal (Fig.3-9), the discrepancy may be due to the differences in area of heat flux integration. Saunders and King (1995) obtained a slightly lower energy transport across A11: $0.5 \mathrm{PW}$.

\section{Indian Ocean heat budget and Pacific-Indonesian Throughflow}

As a consequence of the large inflow of warm water from the PIT (1.36 \pm 0.15 PW energy transport across JADE), the net estimated heat gain over the Indian Ocean is indistinguishable from zero $(0.1 \pm 0.2 \mathrm{PW})$. The heat residual anomalies in individual boxes (Fig.4-2) are also not significantly different from zero. Only in the subtropical box (detail not shown) is there a marginal indication of oceanic heat loss in the surface layer $(0.5 \pm 0.4 \mathrm{PW})$ and heat gain in the second layer $(0.4 \pm 0.6 \mathrm{PW})$ which outcrops almost completely by $32^{\circ} \mathrm{S}$.

The ECMWF energy flux estimates of Siefridt et al. (1999) and Garnier et al. (1999) effectively show no significant heating over the northern part of the Indian basin; neither 
does the NCEP/NCAR re-analysis of Kalnay et al. (1996, shown in Garnier et al. 1999). However, both indicate a cooling of $0.4 \mathrm{PW}$ between $20^{\circ} \mathrm{S}$ and $32^{\circ} \mathrm{S}$. On the other hand, the COADS data of da Silva et al. (1996) and the climatology of Oberhuber (1988) and Hsiung (1985) indicate a warming of about $1.2 \mathrm{PW}$ in the Indian Ocean north of $20^{\circ} \mathrm{S}$. The GCM assimilation computations of Lee and Marotzke (1997, 1998; hereafter LM97,98) which are forced with the Oberhuber (1988) climatology, indicate a consistent heating between $20^{\circ} \mathrm{N}$ and $14^{\circ} \mathrm{S}(0.8 \mathrm{PW})$ and a cooling in the subtropical region $\left(14^{\circ} \mathrm{S}-32^{\circ} \mathrm{S}, 0.3 \mathrm{PW}\right)$. Several other GCM studies based on different surface forcing show heating magnitudes north of $14^{\circ} \mathrm{S}$ that are similar to those of LM97,98 (i.e., their Fig. 19). All GCM-based inversions have a relatively weak or non-existent PIT, so that local atmospheric heating accounts for most of the temperature flux leaving the basin at $32^{\circ} \mathrm{S}$.

The estimate of heat divergence over the Indian Ocean is thus largely determined by the size of the PIT (e.g., Godfrey, 1996). Based on a PIT varying randomly from 1 to $11 \mathrm{~Sv}$, Robbins and Toole (1997) estimated the cnergy flux divergence for the Indian Ocean north of $32^{\circ} \mathrm{S}$ at $0.4 \pm 0.2 \mathrm{PW}$, similar to the result of Hastenrath and Greischar (1993) which was based on a climatological combination of ship observation, radiation measurements and sub-surface temperature.

My lower heat gain over the Indian basin is the consequence of the relatively large PIT temperature flux combined with a relatively weak overturning circulation. Similarly, the energy transport in the South Pacific Ocean is oriented northward because of the large PIT (Fig. 4-2). Most other estimates obtained a weak (and 100\% uncertain) southward transport (see Tsimplis et al. (1998), Fig.17, for a review). Tsimplis et al. (1998) actually obtained northward energy transport at $32^{\circ} \mathrm{S}$ assuming a $10-\mathrm{Sv}$ PIT.

\section{Indo-Pacific energy flux}

To circumvent the uncertainty related to the PIT energy transport, the net meridional temperature transport is traditionally summed over the Pacific and Indian Oceans. Figure 4-5 shows the Indo-Pacific energy fluxes. I find a net heat gain of $1.6 \pm 0.4 \mathrm{PW}$ between $30^{\circ} \mathrm{S}$ and $24^{\circ} \mathrm{N}$, with a $-0.6 \pm 0.2 \mathrm{PW}$ heat loss (largely in the North Pacific) between $24^{\circ} \mathrm{N}$ and $48^{\circ} \mathrm{N}$. Between $30^{\circ} \mathrm{S}$ and $20^{\circ} \mathrm{S}$, the model has a marginal indication of cooling, 


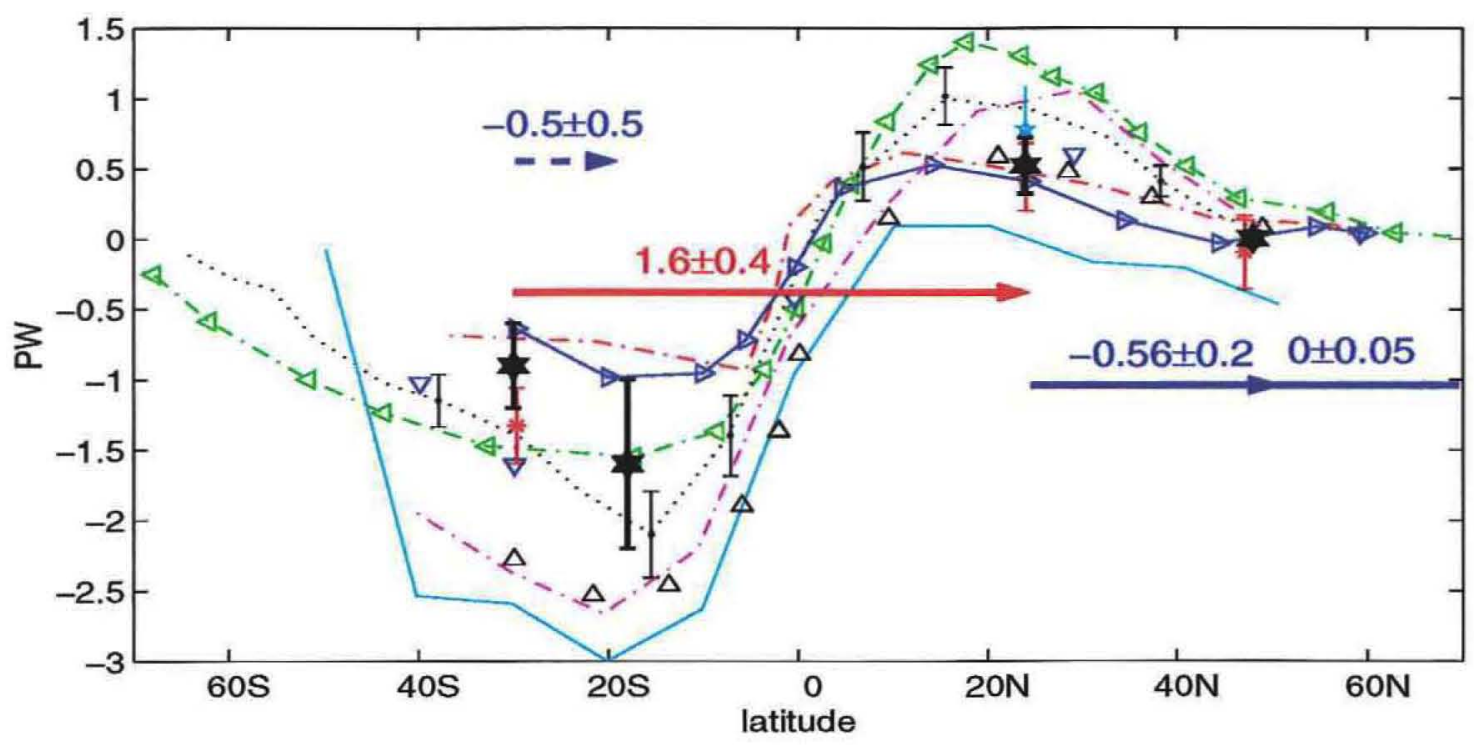

Figure 4-5: Indo-Pacific energy transports and residuals. See Fig. 4-4 for the legend.

$-0.5 \pm 0.5 \mathrm{PW}$.

Those estimates of the net energy transports are in agreement with Macdonald and Wunsch (1996) and Bryden et al. (1991) in the North Pacific within one standard deviation. In the south Indo-Pacific, our southward energy flux is smaller than the Macdonald and Wunsch (1996) estimate, but still compatible within two standard deviations.

The heat divergences obtained by Garnier et al. (1999) and Semtner and Chervin are similar to ours; but all other estimates suggest a larger heat gain of up to $3.5 \mathrm{PW}$ in the tropical band (i.e., Hastenrath, 1982), which are incompatible with our estimates.

\section{Global energy fluxes}

Figure 4-6 shows the global energy transports and residuals. Globally, the ocean heated $(2.3 \pm 0.4 \mathrm{PW})$ between $30^{\circ} \mathrm{S}$ and $24^{\circ} \mathrm{N}$ and cooled poleward of those latitudes, with $-1.7 \pm$ $0.2 \mathrm{PW}$ in the northern hemisphere and $-0.7 \pm 0.3 \mathrm{PW}$ in the southern oceans. The Macdonald and Wunsch (1996) estimate indicated a similar warming, as did the $\mathrm{Hu}(1997)$ isopycnal model. Most other estimates from numerical models and in situ data indicate a larger warming of around 3.5 PW in the tropical band. But the large uncertainties in the ECMWF and NMC model products provided by Keith (1995, green shaded area) indicate 


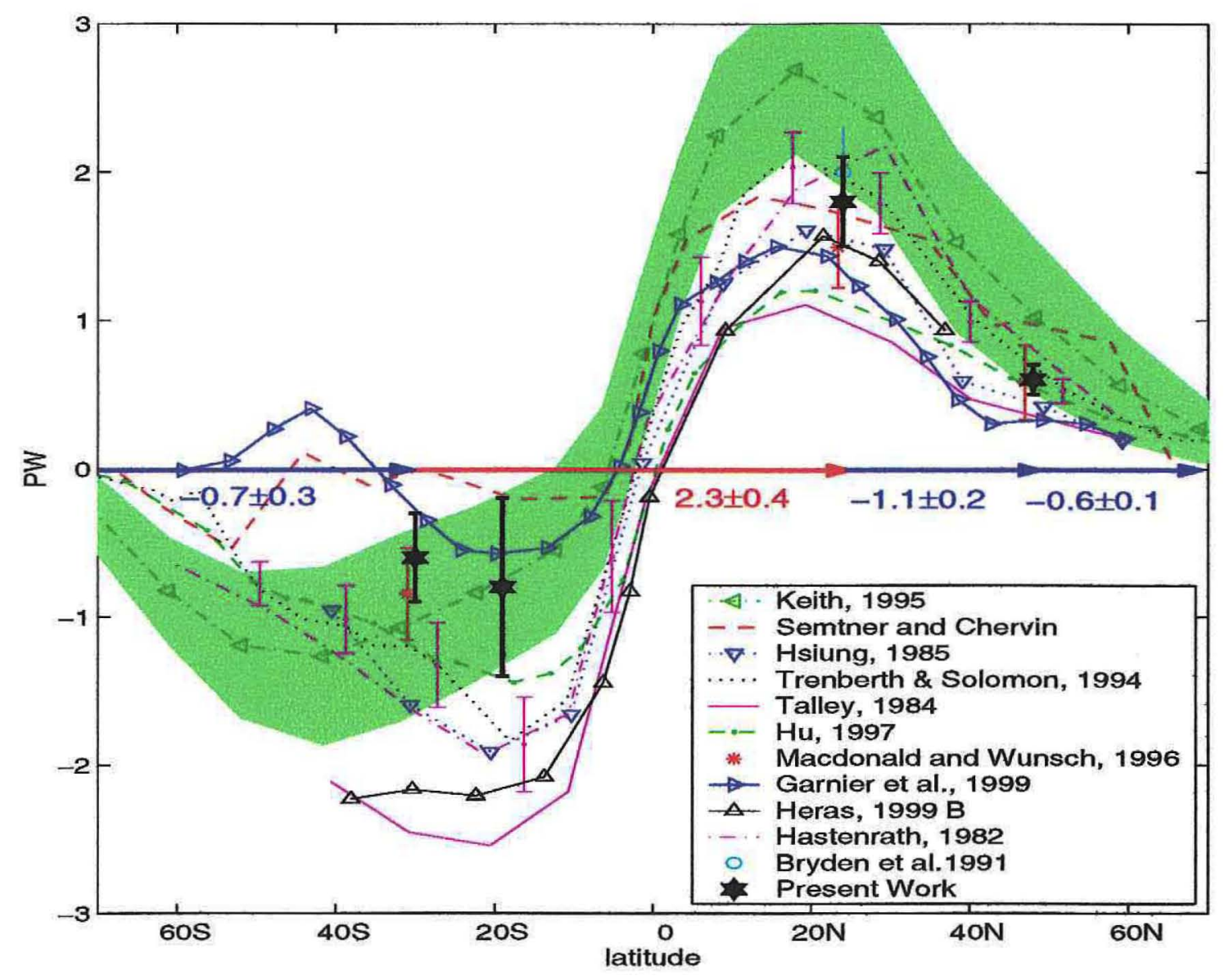

Figure 4-6: Same as Fig.4-4, but for the global energy transports and residuals. The green band indicates the Keith (1995) uncertainties. 
that there are no incompatibilities with our estimates. Garnier et al. (1999), from the same ECMWF model but using the 15-year re-analysis, obtained a much lower oceanic warming at low latitudes than Keith (about 2.5 PW). The Semtner and Chervin estimate is also smaller, with about $1.75 \mathrm{PW}$ warming between $30^{\circ} \mathrm{S}$ and $24^{\circ} \mathrm{N}$.

\section{Conclusions for energy fluxes}

Apart from hydrographic estimates, the existing estimates of oceanic energy transports and ocean-atmosphere energy fluxes span a large range of conflicting values. The degree of agreement between previous estimates and my model varies with location. Generally speaking, the ECMWF 15-year re-analysis (Garnier et al., 1999) is in best agreement with my energy flux estimates over most oceanic regions. Many past estimates were provided with no uncertainties, but are incompatible, within my model's uncertainties, with the present estimates. However, according to the uncertainty estimate of Keith (1995), all meteorologically-based energy flux estimates are consistent with ours within one standard deviation over the northern hemisphere and within two standard deviations over the southern hemisphere. Furthermore, according to Keith (1995), this uncertainty is a lower bound on the actual uncertainty from NWP centers.

On the other hand, our estimate is compatible within error bars with the published estimates from hydrographic sections. Nevertheless, the cooling that we obtain in the Southern Ocean is weaker than most other estimates, resulting in a smaller poleward energy flux at $30^{\circ} \mathrm{S}$. 


\subsection{Export production and nutrient cycle}

Quantification of the global biogenic production in the ocean is one of the major issues in climate prediction. Photosynthesis associated with primary production in the surface layers uses carbon dioxide to form unicellular algae. Carbon is thus fixed, forming organic matter in the surface layers. A large part of the organic matter produced in the surface waters, (also called the total primary production) is recycled in the euphotic zone (regenerated production), with the balance exported towards the deep ocean (export production). This export represents a flux of carbon towards the deep ocean which is believed to control and regulate air-sea $\mathrm{CO}_{2}$ exchanges with a direct implication on climate. Few methods exist to estimate this "export production". Satellite measurements of ocean color provide an estimate of the biomass in upper layers (e.g., Antoine et al., 1996), from which the primary production can be derived. Export production is related to the primary production. (The difference between the export and primary production is the "regenerated production".) But not only is the satellite-derived primary production uncertain, but also its relationship to export production is poorly known, and probably changes with time and location. Export production can apparently reach up to $50-80 \%$ of the primary production during episodic blooms or in high productivity areas; otherwise, it varies in the range 5-10\% (Buesseler, 1998).

The existing measurements of export production from sediment traps vary by one or two orders of magnitude, depending upon region and regime. Lampitt and Antia (1997) reviewed the existing measurements normalized at $2000 \mathrm{~m}$. Using their normalization function, the annual averages exported production at $150 \mathrm{~m}$ is in the range 0.3 to $3 \mathrm{~mol} C$ $\mathrm{m}^{-2} \mathrm{yr}^{-1}$ in all oceanic regions except polar oceans (there is at least $100 \%$ uncertainty from this conversion, K. Buesseler, personal communication, 1999). In polar oceans, the variations are more extreme, with values reported between 0.08 and $5 \mathrm{~mol} \mathrm{C} \mathrm{m} \mathrm{m}^{-2} \mathrm{yr}^{-1}$. But different biases can affect sediment trap results (Siegel and Deuser, 1997), leading to underestimation of the true particle flux by a factor of 3 to 10 (Buesseler, 1991). Export production estimates based upon the ${ }^{234}$ Th method (Michaels et al., 1994; Buesseler, 1998) are about ten times higher, ranging from 1 to $10 \mathrm{~mol} \mathrm{~m}^{-2} \mathrm{yr}^{-1}$ for all oceans excluding 
the polar regions, and from 0.3 to $30 \mathrm{~mol} \mathrm{C} \mathrm{m}^{-2} \mathrm{yr}^{-1}$ in the polar oceans.

On the other hand, export production is directly related to dissolved nutrient fluxes. A flux of particles from the surface to the deep layers creates a sink of dissolved nutrients in the upper layers by consumption and a source in the lower layers through dissolution (also called remineralization). In the hypothetical steady-state, those divergences are balanced by horizontal and vertical transports. Similar divergences may occur as a result of horizontal advection of dissolved organic matter.

In this section, flux divergences of dissolved nutrients are estimated and discussed in terms of particle fluxes and biogeochemical cycles. Redfield ratios of dissolved nutrients are used to convert the results between the different dissolved properties to compare my results with the literature (e.g., Redfield et al., 1963). I use the Anderson and Sarmiento (1994) stoichiometric relation $\mathrm{P} / \mathrm{N} / \mathrm{C} /-\mathrm{O}_{2}=1 / 16 / 117 / 170$. The discussion is started with the silica cycle, where I justify the assumption of quasi-conservation of dissolved silica from surface to bottom over the water column. Following that, nitrate and phosphate flux divergences are analyzed. Oxygen flux divergences, which are tightly related to the biological cycle, are discussed at the end.

\subsubsection{Silica}

Silica is a major nutrient that feeds biological production in the oceans. Siliceous planktonic organisms, mainly diatoms and radiolaria, consume dissolved silica to build their skeletons (e.g., Broecker and Peng, 1982 ). In the opaline form, silica goes through the food chain and ends up in sinking fecal pellets and skeletons. Most of the opal is dissolved in deep waters, although a fraction of the particles are buried on the sea floor. Opal burial occurs mostly in highly productive areas, forming remarkable opaline bands on the sea floor under upwelling areas (Lisitzin, 1972; Aston et al., 1983; Archer, 1993).

The dissolution process within the water column is poorly known. Edmond(1974) suggested that most dissolution takes place on the sea floor. Using sediment traps near Guinea, Takahashi and Honjo (1981) also found that radiolarian shells did not dissolve substantially between about $1000 \mathrm{~m}$ and the sea floor. Sayles et al. (1996) reached a similar conclusion in the Sargasso Sea, where they found that below the first $150 \mathrm{~m}$, where $66 \%$ 
of the dissolution had taken place, no apparent dissolution occurred before particles reach the sea floor. Particles particles reaching the bottom remain on the sea floor for a few hundred years; most eventually remineralize with only a small fraction fully buried and lost to the ocean system. River runoffs are believed to provide an equivalent gain of silica to the ocean (Tréguer et al., 1995)).

These results are uncertain. Not only are they localized to a few places, but also sediment trap measurements can be biased by a factor of 10 (e.g., Michaels et al., 1994). Such studies are complicated by both the diversity of siliceous species and the vertical nature of the data (i.e. along the wire of the mooring) in a situation where horizontal advection and inhomogeneous surface production may bias the measurement (e.g., Buesseler, 1991; Siegel and Deuser, 1997).

Tréguer et al. (1995) and Nelson et al. (1995) established a global budget for the silica in the oceans. Their estimate for the total silica input to the oceans, mainly through river runoff and erosion rate, was close to $200 \mathrm{kmol} \mathrm{Si} \mathrm{s}^{-1}$. They estimated the upper bound for the global primary production of siliceous organisms at $9000 \mathrm{kmol} \mathrm{Si} \mathrm{s}^{-1}$, using existing ${ }^{14} \mathrm{C}$ production estimates combined with silica/carbon ratio in siliceous organisms. Their lower bound estimate, $6400 \mathrm{kmol} \mathrm{Si} \mathrm{s}^{-1}$, was obtained from a model.

\section{Should dissolved silica be conserved over the water column?}

As mentioned in Chapter 1, quasi conservation of dissolved silica over the water column is required as a constraint on the physical circulation. The rational for this constraint is given here.

In the upper $100 \mathrm{~m}$ (the euphotic zone), dissolved silica is almost totally depleted by diatoms and radiolarians. According to Nelson et al. (1995), 50\% of the global opal production is recycled in the euphotic zone, allowing a maximum global particle flux of $4500 \mathrm{kmol} \mathrm{Si} \mathrm{s}^{-1}$ to enter the deep layers (those numbers are highly uncertain). As noted above, some of these particles are buried on the sea floor. The total burial/production ratio is, on average, $3 \%$ and varies strongly with location. The burial is believed to be almost, negligible in the large oligotrophic regions (e.g., Tréguer et al. 1995) . Similarly, in high-productivity regions such as the Southern Oceans, recent isotopic measurements from 
the JGOFS Antarctic program indicate that little burial has occurred for at least the last 5000 years (a maximum of $5 \%$ of the total production, F. Sayles, personal communication, 1999). This result contradicts previous beliefs that up to $25 \%$ of the total production was buried there (De Master, 1991).

Globally, the net sources and sinks of silica thus appear to be much smaller than the biogenic fluxes. The global sink of silica estimated from sediment data is about $220 \mathrm{kmol} \mathrm{Si} \mathrm{s}^{-1}$, which is close to the total input from river runoff, at $160 \mathrm{kmol} \mathrm{Si} \mathrm{s}^{-1}$ (Tréguer et al., 1995). Other sources of silica, such as hydrothermal activity, basaltic erosion and eolian inputs, are probably even smaller $\left(40 \mathrm{kmol} \mathrm{Si} \mathrm{s}^{-1}\right.$ in total, Tréguer et al., 1995) although little is known about them. Regionally, those sources and sinks may be more important, but still smaller than biological fluxes.

Two additional processes may lead to non-conservation of dissolved silica between hydrographic sections. Namely, the erosion of the sea floor during a transient period, or the horizontal advective transport of siliceous particles across an oceanic section.

Is sea floor erosion able to provide a large flux? In the steady state, it is believed that silica is buried in the sediments, i.e., there is a net opal flux to sediments. The burial can lead to a sediment accumulation up to $17 \mathrm{~cm} / \mathrm{Kyr}$ in the Southern Oceans (De Master, 1981). In my view, a net erosion would have to correspond to the reversal of the accumulation during a hypothetical episodic (on climate time scales) event. A $100 \mathrm{kmol} \mathrm{Si} \mathrm{s}^{-1}$ source over an area such as the North Atlantic between $24^{\circ} \mathrm{N}$ and $36^{\circ} \mathrm{N}$ corresponds to an area average erosion rate of $0.4 \mathrm{~mol} \mathrm{Si} \mathrm{yr}^{-1} \mathrm{~m}^{-2}$. In this case, taking a sediment density of $0.4 \mathrm{~g} \mathrm{~cm}^{-3}$ (De Master, 1981), with a $1.5 \%$ opal content (Sayles et al., 1996 , p.20), the sea floor erosion rate would be

$$
\begin{aligned}
& 0.4 \mathrm{~mol} \mathrm{Si} \mathrm{yr}{ }^{-1} \mathrm{~m}^{-2} \times 60 \frac{\mathrm{g} \mathrm{Si}}{\mathrm{mol} \mathrm{Si}} \times \frac{1}{0.015} \frac{\mathrm{g} \mathrm{Tot}}{\mathrm{g} \mathrm{Si}} \times \frac{1}{0.4} \frac{\mathrm{cm}^{3}}{\mathrm{~g} \mathrm{Tot}^{-}} \\
& =4 \mathrm{~m} / \mathrm{Kyr} .
\end{aligned}
$$

An erosion rate of $4 \mathrm{~m} / \mathrm{Kyr}$ (meter per Kilo-year) seems unreasonably large. Besides, direct measurements of the benthic silica flux with benthic chambers indicates maximum fluxes of $0.036 \mathrm{~mol} \mathrm{Si} \mathrm{yr}{ }^{-1} \mathrm{~m}^{-2}$ near Bermuda (Sayles et al., 1996). Their calculation based on the Si gradient in the pore water samples near the sediment-water interface led to the 
same result. In the Equatorial Pacific, similar measurements indicated larger fluxes (up to $0.25 \mathrm{~mol} \mathrm{SiO}_{2} \mathrm{yr}^{-1} \mathrm{~m}^{-2}$, McManus et al., 1995). The largest dissolution rates are found near Antarctica, with up to $1.5 \mathrm{~mol} \mathrm{SiO}_{2} \mathrm{yr}^{-1} \mathrm{~m}^{-2}$ (F. Sayles, personal communication, 1999). Still, measurements indicate that those fluxes from the sea floor derive from the opal rain, thus implying net deposition and no net erosion to the sea floor. As a result, the net source of silica near the sea floor should be approximately in balance with the opal rain.

Can siliceous particles be advected across sections? Horizontal advection of siliceous particles may create an apparent source or sink in the dissolved silica budget. Values of vertically integrated rates of biogenic silica production vary between $0.2 \mathrm{~mol} \mathrm{Si} \mathrm{yr}{ }^{-1} \mathrm{~m}^{-2}$

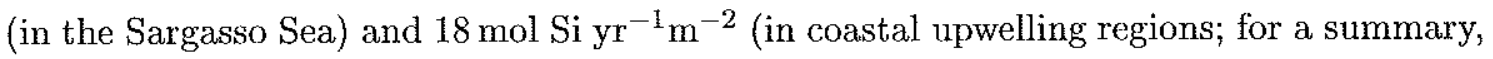
see Nelson et al., 1995).

If the particles rained with a downward velocity of $100 \mathrm{~m} /$ day (Banse, 1990 ), the average particulate silica concentration over the water column would range from $5 \times$ $10^{-3} \mu \mathrm{mol} / \mathrm{kg}$ to $500 \times 10^{-3} \mu \mathrm{mol} / \mathrm{kg}$. Multiplied, for instance, by the Florida Current flow $\left(30 \mathrm{~Sv}\right.$ ), the silica flux would range 0.15 to $15 \mathrm{kmol} \mathrm{Si} \mathrm{s}^{-1}$. Taking into account that $50 \%$ of the particles are permanently recycled in the upper layer would increase the par ticle concentration in the fast-moving layers-and thus the flux by a few fold. Honjo and Manganini (1993) argued for a slower downward particle velocity of $50 \mathrm{~m} / \mathrm{day}$, which, for the same production, would double the particle concentration and the resulting horizontal flux.

The particle flux across a whole section is difficult to estimate, but it is likely to be the same order of magnitude as the above estimated Florida Current flux because the net flux is likely dominated by regions of simultaneous high productivity and strong currents. Particle advection thus appears to be, within our knowledge, smaller than our reference uncertainty of $100 \mathrm{kmol} \mathrm{Si} \mathrm{s}^{-1}$ (i.e., Section 2.2.4), although the magnitude is not so weak that it can be completely ignored. Note that within the steady state assumption, strong particle advection would be associated with a large silica depletion on one side of the section and an enrichment on the other side.

Seasonal effect may also be an issue. Williams and Follows (1998) found that important 
seasonal correlation between nitrate and Ekman transports at the edge of the subtropical gyre may produce "eddy" fluxes that are not captured by analyses based on annual averages. But for nitrate this effect is weak in comparison to the magnitude of our uncertainties (see the discussion below, page 168), and it is anticipated to be small for the silica too.

To first order therefore, sea floor erosion and particle advection do not appear to create large sources or sinks of dissolved silica. Other sources or sinks are a priori relatively small. Within present knowledge, dissolved silica can thus be taken as conserved within $\pm 100 \mathrm{kmol} \mathrm{Si} \mathrm{s}^{-1}$ over large oceanic areas such as the North Atlantic between $24^{\circ} \mathrm{N}$ and $36^{\circ} \mathrm{N}$. (Practically, uncertainties are larger due to oceanic variability and measurement noise, Section 2.6.) (For practical reasons, no constraint was imposed to the global silica budget.)

\section{Silica flux divergences}

Silica, and other nutrient flux divergences were examined in each box. Most of the residuals in individual layers have a $100 \%$ uncertainty. However, when a sign change was noticed between the surface and the deep layers, nutrient residuals were summed separately surface/deep. In boxes where residuals within individual layers were more than $150 \%$ uncertain, the summation was done over all layers (top-to-bottom). Figure 4-7 shows those silica fluxes and divergences. (Note that for technical reasons, and in contrast with all other tracers, silica divergences are not corrected for mass divergences, i.e., the anomaly formulation was not used (Section 1.2.4, page 24). As a result, some of the silica divergences may be associated with mass imbalances).

Silica fluxes (arrows) are directed southward within the Atlantic Ocean, and poleward elsewhere. In the Atlantic, the flux is of about $-150 \pm 75 \mathrm{kmol} \mathrm{Si} \mathrm{s}^{-1}$. In other regions, meridional fluxes are more uncertain. Silica residuals are indistinguishable from zero within one standard deviation in most places, i.e., the conservation constraint was met. But there are exceptions in the central Pacific and subtropical and tropical Indian Oceans where the residuals are between one and two standard deviations. In the central Pacific Ocean, there is an apparent source of dissolved silica $\left(+0.35 \pm 0.15 \mathrm{~mol} \mathrm{SiO}_{2} \mathrm{yr}^{-1} \mathrm{~m}^{-2}\right)$ while 


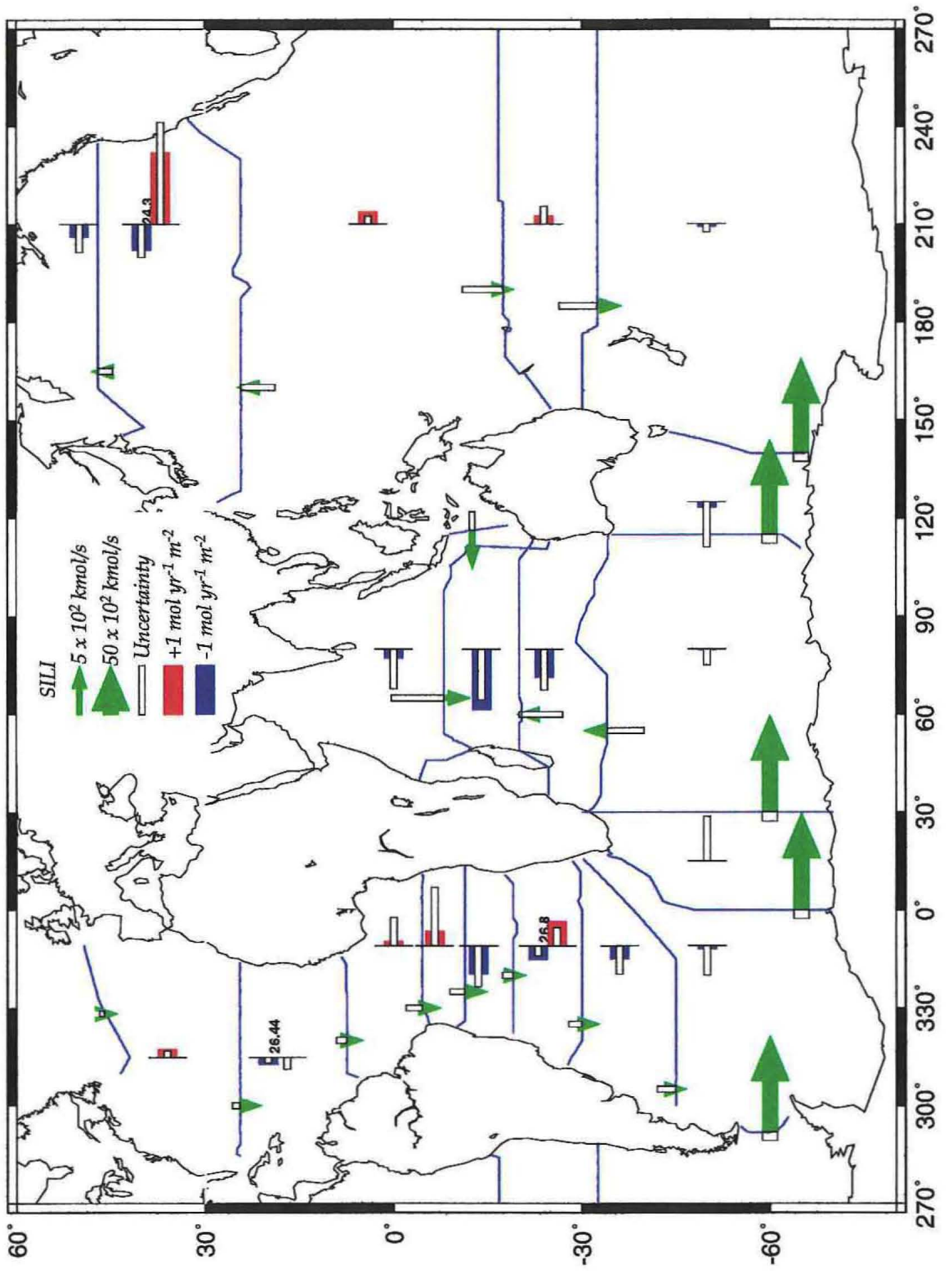

Figure 4-7: Global dissolved silica transports and residuals. The length of each arrow corresponds to the silica transports between continents. The white boxes behind each arrow indicate the uncertainty (one standard deviation). Between sections, silica divergences are indicated by the full boxes, either top-to-bottom (single box) or surface/deep (double box). In the later case, the number indicates the interface (in neutral density units). Residuals are not given where the uncertainty exceeds $150 \%$. 
a sink is found in the Indian Ocean $\left(-0.28 \pm 0.24 \mathrm{~mol} \mathrm{SiO}_{2} \mathrm{yr}^{-1} \mathrm{~m}^{-2}\right)$. This imbalance may be a consequence of the high Pacific-Indonesian Throughflow. (The model's PIT transports $400 \pm 300 \mathrm{kmol} \mathrm{Si} \mathrm{s}^{-1}$ to the west, implying a source in the Pacific and a sink in the Indian Ocean that may be erroneous-although both are indistinguishable from zero.)

The Southern Ocean is an uncertain net sink of silica at $-480 \pm 380 \mathrm{kmol} \mathrm{Si} \mathrm{s}^{-1}$ (equivalent to $-0.18 \pm 0.15 \mathrm{~mol} \mathrm{SiO}_{2} \mathrm{yr}^{-1} \mathrm{~m}^{-2}$ overall). This rate is greater than the largest burial rates measured in the high production regions by the JGOFS Antarctica program: $0.05 \mathrm{~mol} \mathrm{SiO}_{2} \mathrm{yr}^{-1} \mathrm{~m}^{-2}$ (F. Sayles, personal communication, 1999).

In most of the temperate regions, there is a depletion of silica in the upper layers ranging from $-0.1 \pm 0.05$ to $-1 \pm 0.6 \mathrm{~mol} \mathrm{SiO}_{2} \mathrm{yr}^{-1} \mathrm{~m}^{-2}$, with a corresponding source in the underlying deep layers. Although highly uncertain, this residual structure corresponds to what one expects from silica consumption and opal dissolution.

The global average production rate of silica was estimated to be $0.6 \mathrm{~mol} \mathrm{SiO} \mathrm{yr}^{-1} \mathrm{~m}^{-2}$ by Nelson et al. (1995), with variations between 0.2 and $8.3 \mathrm{~mol} \mathrm{SiO}_{2} \mathrm{yr}^{-1} \mathrm{~m}^{-2}$, the highest production rates occurring in coastal upwelling areas. Between $10 \%$ and $50 \%$ (e.g., Honjo and Manganini, 1993; Nelson et al., 1995) of this is exported to the deep layers, resulting in an opal dissolution rate ranging 0.02 to $4 \mathrm{~mol} \mathrm{SiO}_{2} \mathrm{yr}^{-1} \mathrm{~m}^{-2}$. Lampitt and Antia (1997) reported opal rain fluxes from particle traps in the same range $\left(0.1\right.$ to $1.2 \mathrm{~mol} \mathrm{SiO}_{2} \mathrm{yr}^{-1} \mathrm{~m}^{-2}$, excluding polar oceans, where low export production attains $0.01 \mathrm{~mol} \mathrm{SiO}_{2} \mathrm{yr}^{-1} \mathrm{~m}^{-2}$ ). The magnitude of our large-scale silica sources in the deep layers in temperate regions is thus within the previously measured range.

\subsubsection{Nitrate and Phosphate}

Figs. 4-8 and 4-9 show the dissolved nitrate and phosphate net fluxes and residuals. As for silica, residuals of dissolved inorganic nutrients were integrated, either top-to-bottom (single red or blue box) or within surface and deep layers if there was a coherent sign change. In contrast with silica, nitrate and phosphate residuals are estimated from anomaly equations, i.e., corrected for mass imbalances. Net fluxes of nitrate and phosphate (arrows) are uncertain overall. There is an indication of southward nitrate flux from the Atlantic Ocean at $45^{\circ} \mathrm{S}$ (A11) (i.e., a nitrate divergence of $340 \pm 120 \mathrm{kmol} \mathrm{N} \mathrm{s}^{-1}$ between $48^{\circ} \mathrm{N}$ and $45^{\circ} \mathrm{S}$ ) 
as well as phosphate $\left(16 \pm 8 \mathrm{kmolP} \mathrm{s}^{-1}\right) .70 \%$ of the corresponding nutrient regeneration occurs in the South Atlantic between $30^{\circ} \mathrm{S}$ and $45^{\circ} \mathrm{S}$.

In the combined tropical Atlantic and "Rio" $\left(19^{\circ} \mathrm{S}-30^{\circ} \mathrm{S}\right)$ boxes, residuals indicate a depletion of nutrients in the surface layers and a source in the deep layers (Fig. 4-8). This same feature is present in the Central and North Pacific. All residuals are highly uncertain but this pattern interestingly corresponds to what would be expected from a hypothetical particle transport that consumes nutrients at the surface and restores them in the deep layers through remineralization. In the rest of the Ocean, the residuals were too incoherent and too uncertain to make this distinction. Net sources or sinks of nitrate and phosphate are found in several oceanic regions, noticeably in the North Atlantic $\left(0.1 \pm 0.1 \mathrm{molN} \mathrm{m}^{-2} \mathrm{yr}^{-1}\right)$. Such a source was also found by Rintoul and Wunsch (1991) using older data, between $24^{\circ} \mathrm{N}$ and $36^{\circ} \mathrm{N}$ (versus $24^{\circ} \mathrm{N}$ and $48^{\circ} \mathrm{N}$ here). Rintoul and Wunsch (1991) found that the circulation derived from the older data could not be made compatible with nitrate conservation. The apparent source led them to suggest the advection of significant dissolved organic matter into the region, where it subsequently remineralized.

Nitrogen fixation is another possible explanation for the apparent sources. However, phosphate (Fig. 4-9) exhibits a similar source in the North Atlantic $\left(0.02 \pm 0.007 \mathrm{~mol} \mathrm{P} \mathrm{m}^{-2} \mathrm{yr}^{-1}\right)$, and no equivalent to fixation is possible. In general, phosphate residuals are similar to nitrate residuals (Figs. 4-8 and 4-9). A few exceptions exist (e.g., south of Africa), but the differences are too uncertain to draw conclusions implying different processes. Consequently, the nutrient divergences that I find are likely to be due to either advection of dissolved organic matter, or to an alias of the seasonal cycle in the hydrographic sections.

The nitrate and phosphate residuals were integrated over latitude bands (Table 4.4). Both exhibit similar features, with about $100 \%$ uncertainty. High latitude oceans experience nitrate and phosphate sources while the tropical band is a sink. These inferred sources and sinks may be the result of advection of dissolved organic matter, or temporal aliasing of the nutrient fluxes across the sections, but all are indistinguishable from zero. Dissolved nitrate and phosphate are both balanced globally.

Net sources of dissolved nutrients in the deep layers range from $0.5 \pm 0.24 \mathrm{~mol} \mathrm{~N} \mathrm{~m}^{-2} \mathrm{yr}^{-1}$ (tropical North Atlantic) to $0.7 \pm 0.2 \mathrm{~mol} \mathrm{~N} \mathrm{~m}^{-2} \mathrm{yr}^{-1}$ (South Atlantic). Moored sediment 


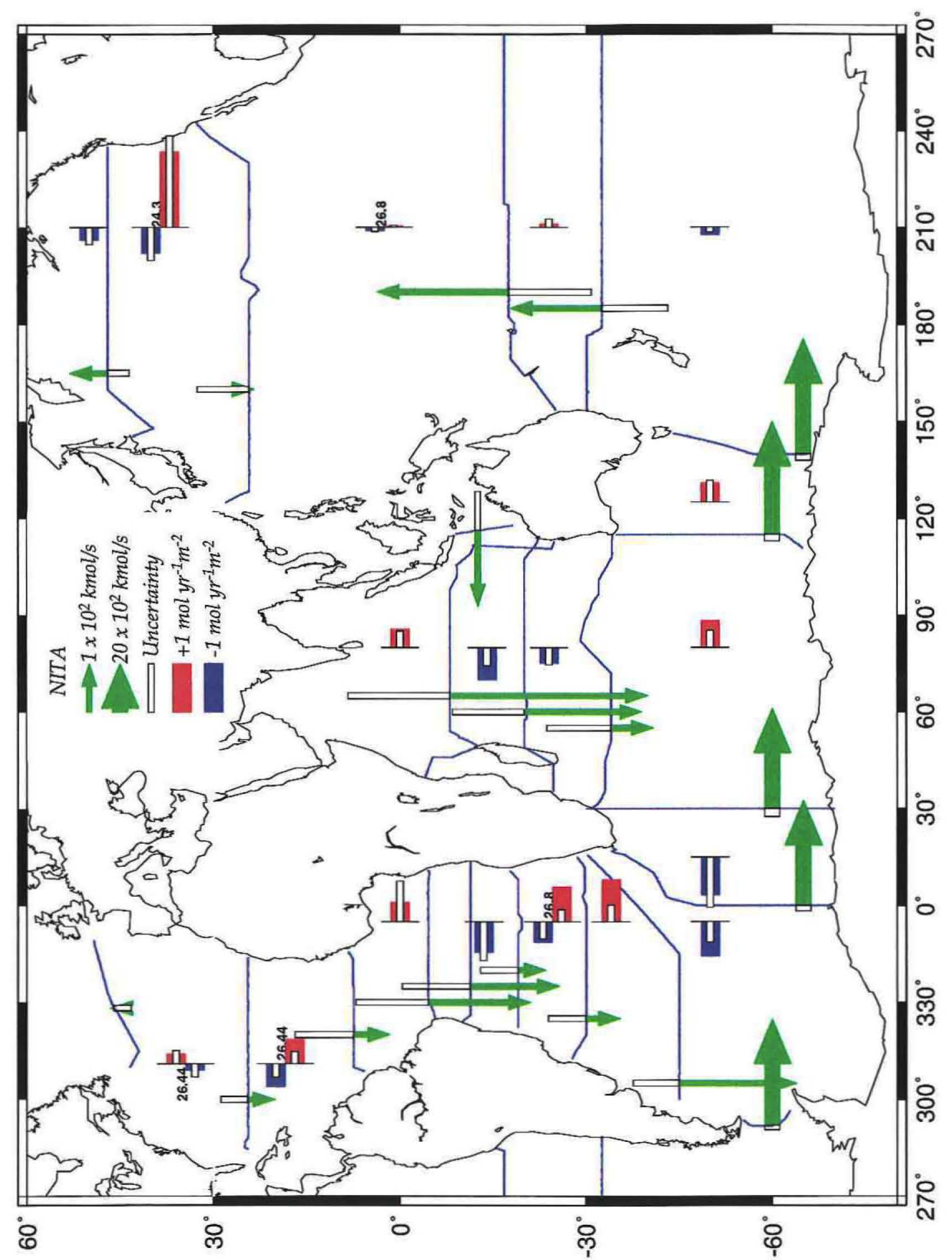

Figure 4-8: Same as Fig. 4-7, but for dissolved nitrate. 


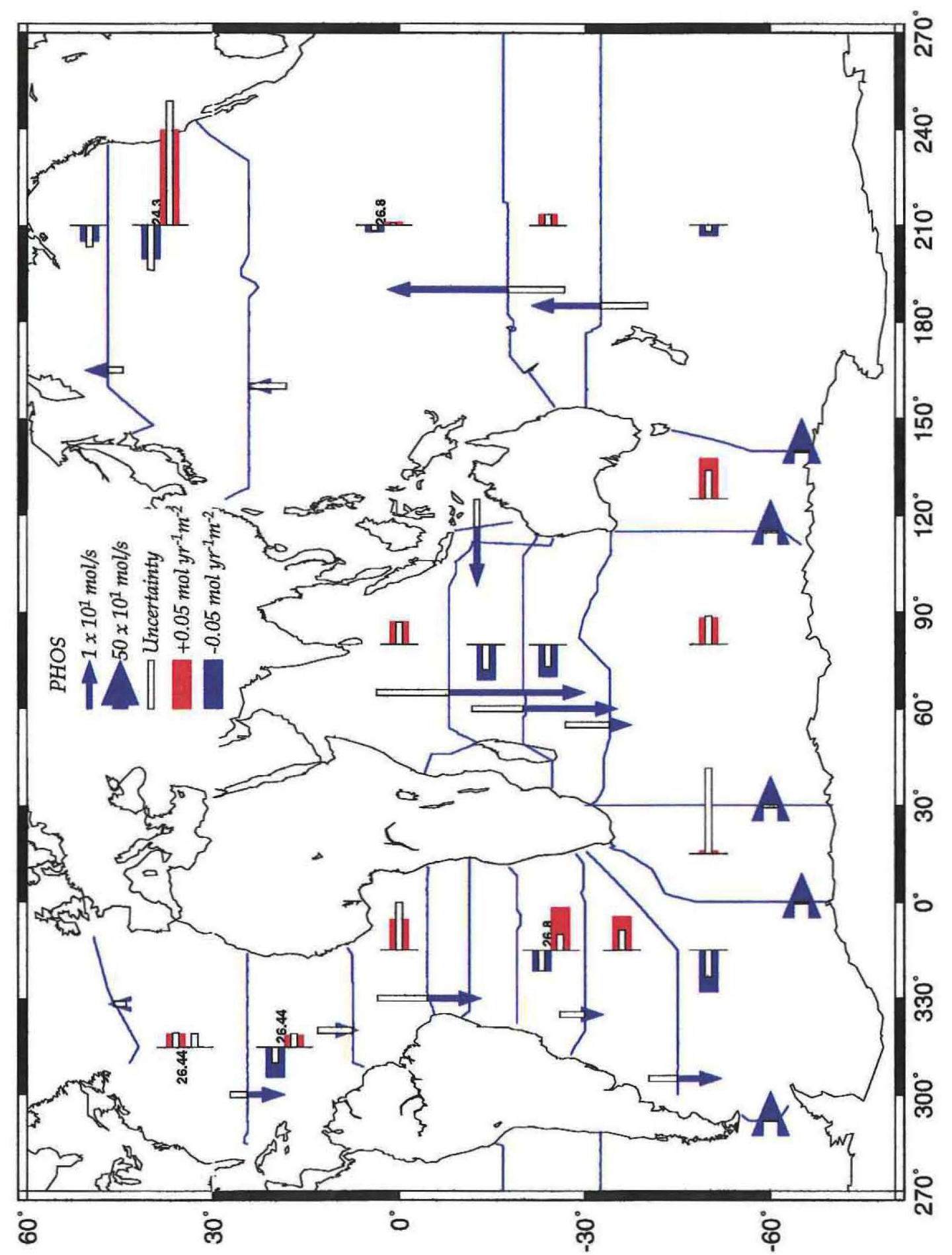

Figure 4-9: Same as Fig. 4-7, but for dissolved phosphate.

trap measurements of particle export from various oceanic regions are of the same order, ranging from 0.04 to $0.4 \mathrm{molN} \mathrm{m}^{-2} \mathrm{yr}^{-1}$ (Lampitt and Antias, 1997). Isotopic mea- 


\begin{tabular}{|l|c|c|}
\hline Latitude & Nitrate $\left(\mathrm{kmol} \mathrm{N} \mathrm{s}^{-1}\right)$ & Phosphate $\left(\mathrm{kmol} \mathrm{P} \mathrm{s}^{-1}\right)$ \\
\hline $24^{\circ} \mathrm{N}-48^{\circ} \mathrm{N}$ & $100 \pm 110$ & $10 \pm 8$ \\
\hline $30^{\circ} \mathrm{S}-24^{\circ} \mathrm{N}$ & $-90 \pm 200$ & $-13 \pm 13$ \\
\hline south of $30^{\circ} \mathrm{S}$ & $100 \pm 250$ & $10 \pm 15$ \\
\hline Global total & $40 \pm 300$ & $-3 \pm 20$ \\
\hline
\end{tabular}

Table 4.4: Nitrate and phosphate residuals integrated globally over latitude bands.

surements based on ${ }^{234} \mathrm{Th}$ suggest that sediment traps on sediment traps may underestimate the actual particle flux by a factor of up to 10 (e.g., Michaels et al., 1994; Buesseler, 1998). Export production estimates from isotopic measurements in temperate and tropical oceanic regions range from 0.05-1.4 mol $\mathrm{m}^{-2} \mathrm{yr}^{-1}$. New production ${ }^{1}$ estimates are in the same range, with about $0.5 \mathrm{~mol} \mathrm{~N} \mathrm{~m}^{-2} \mathrm{yr}^{-1}$ (e.g., Jenkins and Goldman, 1985), which is in rough agreement with the nutrient sources and sinks found in the surface layers in the Atlantic, namely, $-0.4 \pm 0.3 \mathrm{~mol} \mathrm{~N} \mathrm{~m}^{-2} \mathrm{yr}^{-1}$ in the South Atlantic and $-0.5 \pm 0.25 \mathrm{~mol} \mathrm{~N} \mathrm{~m}^{-2} \mathrm{yr}^{-1}$ in the tropical North Atlantic.

The consistency between local measurements and large scale averages may be fortuitous. For instance, a single layer may outcrop so that both consumption and remineralization occur in the same box with the two regimes cancelling. Also, neutral surfaces do not correspond to the bottom of the sun-lit layer (or 150-m, the level to which sediment trap measurements were normalized). Nevertheless, the magnitudes of the nitrate residuals are in close agreement with independent measurements. Given the large uncertainties, the inverse model estimate provides at best an upper bound for the average remineralization process. Overall, nitrate residuals (including deep/surface and net in a box) are in the range $-0.8 \pm 1 \mathrm{~mol} \mathrm{~N} \mathrm{~m}^{-2} \mathrm{yr}^{-1}$ to $-1.8 \pm 1.8 \mathrm{~mol} \mathrm{~N} \mathrm{~m}^{-2} \mathrm{yr}^{-1}$.

\subsubsection{Discussion of nutrient fluxes and divergences}

Despite the large uncertainties, the magnitude of the maximum export production agrees closely with independent measurements from moored sediment traps and isotopic data. A (possible) signature of time-mean, large-scale average export production is found for silica,

\footnotetext{
${ }^{1}$ New production is defined here as the amount of production deriving from the nutrient supply from the deep layers in a hypothetical one-dimensional balance. In the absence of horizontal advection, new production is equivalent to export production.
} 
nitrate and phosphate in several temperate regions, with the exception of the Indian Ocean. Net sources and sinks of nutrients are found in several regions too, but all are indistinguishable from zero within a two-standard-deviation uncertainty. Slight differences between nitrate and phosphate flux divergences were noted. These are, in general, not significant, thus providing no evidence for nitrogen fixation or denitrification. In the Indian Ocean, there is an (uncertain) source of nutrients in the north and an (uncertain) sink in the tropical and subtropical regions. It is not clear whether this pattern is an artifact of an exceptionally large Pacific Indonesian Throughflow on JADE 1989 that created episodic nutrient imbalances, or a consequence of particle advection across the sections.

Imbalances may also relate to alias of the seasonal or interannual variability in the biological activity (particle flux) and/or the advective nutrient fluxes. At the edge of the subtropical gyre, Williams and Follows (1998) suggested that seasonal correlations between nutrient concentrations and Ekman transports could be important. But their biases in the nitrate fluxes are of order $4 \mathrm{kmol} \mathrm{N} \mathrm{s}^{-1}$ (McLaren, personal communication, 1999), which is 10 times smaller than my uncertainties.

Time variability in biological activity can also introduce additional biases when estimating a time-average because of the different time scales between production and dissolution. For instance, Deuser et al. (1995) found a 50\% change in the opal export flux to the deep Sargasso Sea between 1978 and 1991. Such a change should be paralleled in the consumption rate, but, if the opal residence time is of order 100 year (Sayles et al., 1996), the change would not be seen in the dissolution on the sea floor. 


\subsection{Oxygen fluxes and divergences}

\subsubsection{The oxygen cycle}

The oceanic oxygen cycle is closely related to production and to the $\mathrm{CO}_{2}$ cycle through photosynthesis and remineralization. Photosynthesis produces oxygen and consumes $\mathrm{CO}_{2}$, while remineralization consumes oxygen. But in contrast with nutrients, the oceanic oxygen cycles include important air-sea fluxes. The amount of oxygen that is pumped or outgassed by the ocean depends upon the partial pressure of oxygen which, in the ocean, is controlled by (1) temperature and, to a minor extent, salinity (e.g., Garcia and Gordon, 1992); (2) biological production and respiration; and, locally (3) oceanic transport and upwelling. Control number (1) is called the "solubility pump" (e.g., Stephens et al., 1998): when water is warmed, partial pressure is increased and oxygen (and $\mathrm{CO}_{2}$ ) are released to the atmosphere. The opposite happens when water is cooled. Control (2) is called the "biological pump", as it consumes oxygen at depth and produces oxygen at the surface.

Depending upon the oceanic region, the solubility pump, the biological pump and the oceanic transports have either reinforcing or opposing effects (e.g., Keeling and Peng, 1995). For instance, in equatorial upwelling regions, where productivity is limited by other factors than nutrient supply, surface warming suggests (provided that the surface oxygen concentration is saturated) an oxygen efflux (ocean $\rightarrow$ atmosphere) while upwelling of low-oxygen, cold deep waters creates an influx. On the other hand, in subtropical gyres, warming, productivity and advection create an effux while at high latitudes cooling and convection both imply an influx.

While several studies address the seasonal cycle of oceanic oxygen, relatively few publications address the average flux on longer time scales. Stephens et al. (1998) compared the time-average oxygen and $\mathrm{CO}_{2}$ concentration and fluxes using ocean biological general circulation models, an atmospheric model and atmospheric observations from 9 stations. They suggested that the ocean GCMs (combined with an atmospheric tracer model) underestimated the southward transports of oxygen and $\mathrm{CO}_{2}$, yielding a large gain (air $\rightarrow$ sea) of oxygen in the Southern Ocean which they suggested was not present in the observations. 
As presented below, my analysis indeed suggests important oxygen air $\rightarrow$ sea flux in the Southern Ocean.

\subsubsection{Oxygen fluxes and divergences}

Figure 4-10 shows the net fluxes and residuals of dissolved oxygen from my standard solution. In contrast with the nutrients, oxygen fluxes and divergences are significantly different from zero in many regions. There is a large influx (atmosphere to ocean) of oxygen in polar and temperate regions and a loss in tropical regions. As a result, oxygen fluxes are directed equatorward almost everywhere (except in the Indian Ocean-because of the oxygen transport associated with the Indonesian Throughflow).

Analysis of the flux divergences (also called influx or efflux, in contrast with flux which is used for horizontal transport across sections) show that in the $24^{\circ} \mathrm{N}-48^{\circ} \mathrm{N}$ latitude band (globally) there is an oxygen influx of $1050 \pm 600 \mathrm{kmol} \mathrm{O}_{2} \mathrm{~s}^{-1}$. North of $48^{\circ} \mathrm{N}$, there is an additional influx of $2400 \pm 650 \mathrm{kmol} \mathrm{O}_{2} \mathrm{~s}^{-1}$. At lower latitudes $\left(24^{\circ} \mathrm{N}-30^{\circ} \mathrm{S}\right)$, oxygen is consumed or/and restored to the atmosphere at a rate of $-7200 \pm 800 \mathrm{kmol} \mathrm{O}_{2} \mathrm{~s}^{-1}$, while in the Southern Ocean there is an influx of the same magnitude as the northern counterpart, $3400 \pm 700 \mathrm{kmol} \mathrm{O}_{2} \mathrm{~s}^{-1}$. Figure 4-11 shows those sources and sinks (blue columns). The global oxygen budget is in balance within the uncertainty $\left(-300 \pm 800 \mathrm{kmol} \mathrm{O}_{2} \mathrm{~s}^{-1}\right)$.

Locally, net loss of oxygen reaches values of up to $8 \pm 1 \mathrm{~mol} \mathrm{O}_{2} \mathrm{~m}^{-2} \mathrm{yr}^{-1}$ in the South Atlantic. In this particular region the oxygen loss occurs over the first $1000 \mathrm{dbars}$, consistent with remineralization. (Note that if particles are created and remineralized in the same oceanic region, oxygen consumption in the subsurface layers would correspond, in the steady state, to an equivalent oxygen production and possibly outgassing in the surface layers through photosynthesis. By conservation of oxygen, a net oxygen utilization observed in a given oceanic region, even at depth, ultimately corresponds to outgassing.)

In the North Atlantic, South Atlantic and North Pacific temperate regions, distinctive regimes can be identified in surface and deep layers, with an oxygen gain in the surface layers, associated with atmospheric influx and, possibly, a contribution from photosynthesis. In deeper layers, an uncertain loss is found, probably associated with remineralization. In layers with oxygen gain, there is no correlation with nutrient loss and the biological pump 


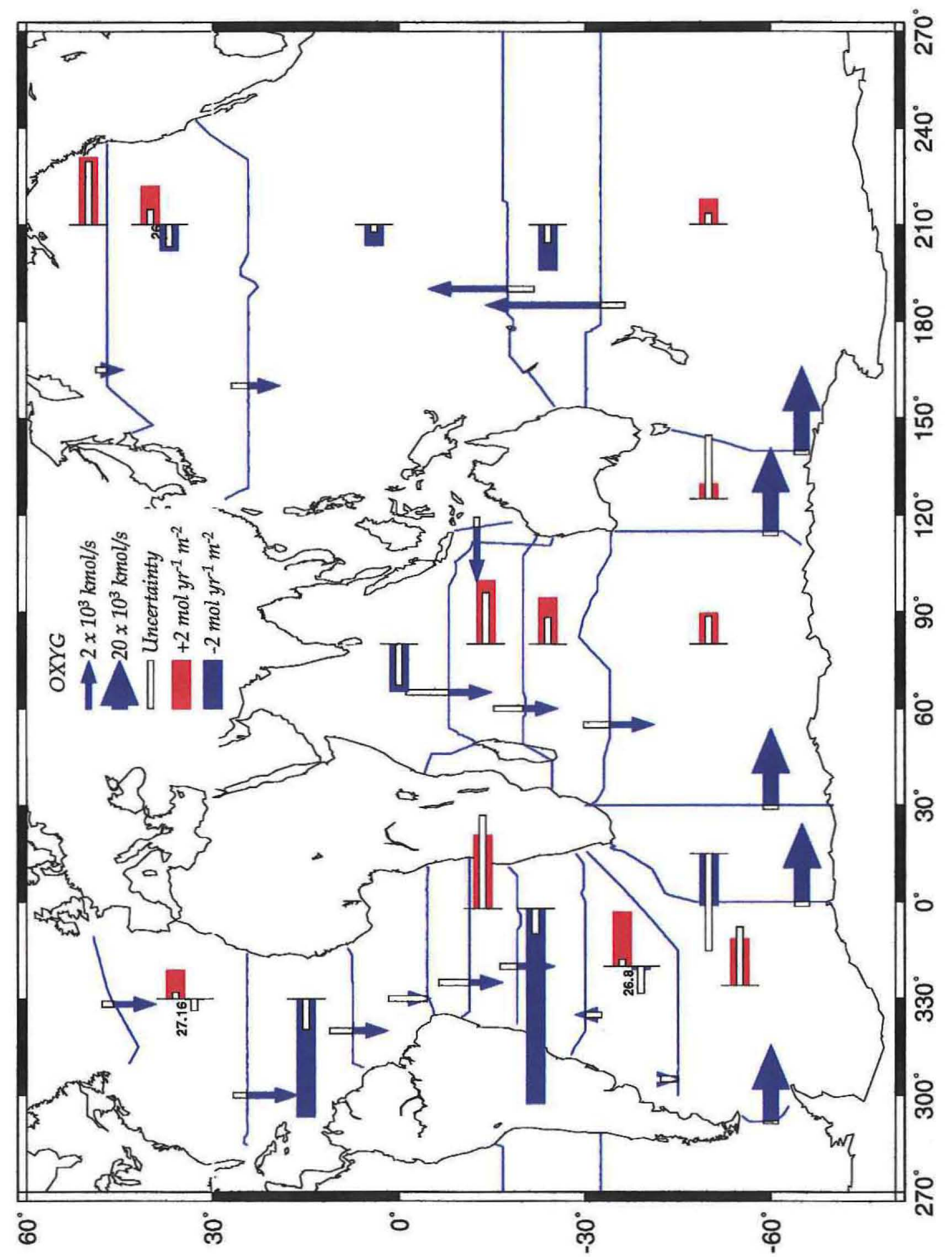

Figure 4-10: Dissolved oxygen fluxes and flux divergences. The caption is the same caption as for nutrients (Fig. 4-7), i.e., residuals were summed individually in deep/shallow layers when the distinction was possible, top-to-bottom otherwise. 


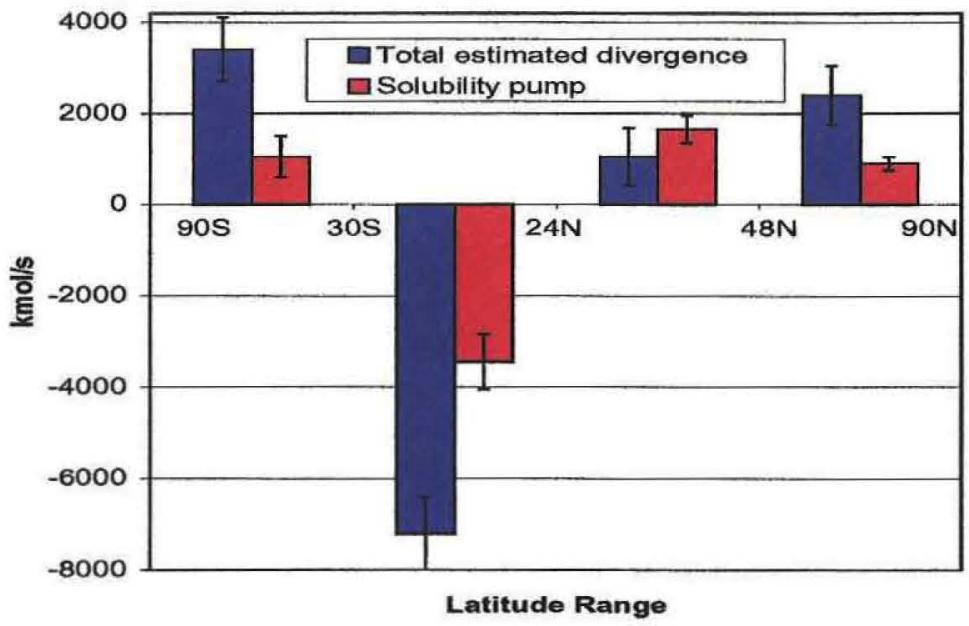

Figure 4-11: Global oxygen sources and sinks for selected latitude ranges. The blue bars show the inverse model estimate, while the red bars correspond to the hypothetical contribution from the solubility pump, estimated from the energy flux divergences $\left(-1500 \mathrm{kmol} \mathrm{O}_{2} \mathrm{~s}^{-1} / \mathrm{PW}\right.$, Holfort et al., 1999).

is probably masked by the solubility pump. In deeper layers (e.g., in the South Atlantic, $19^{\circ} \mathrm{S}-30^{\circ} \mathrm{S}$ ), oxygen utilization is correlated with nutrient input.

Table 4.5 shows a list of previous estimates of oxygen utilization rate (OUR) and production from different methods (adapted from Sarmiento et al., 1990). The OUR estimate from Sarmiento et al. (1990) is $2.4-2.5 \mathrm{~mol} \mathrm{O}_{2} \mathrm{~m}^{-2} \mathrm{yr}^{-1}$, when derived from tritium inventories, and $8.5 \pm 0.8 \mathrm{~mol} \mathrm{O}_{2} \mathrm{~m}^{-2} \mathrm{yr}^{-1}$ when derived from ${ }^{228} \mathrm{Ra}$ measurements. Those authors argue that the ${ }^{228} \mathrm{Ra}$-values are more reliable than those from the tritium inventory. The magnitude of oxygen loss that I obtained in the South Atlantic $\left(20^{\circ} \mathrm{S}-30^{\circ} \mathrm{S}\right)$ is of the same size $\left(\simeq 8 \mathrm{~mol} \mathrm{O}_{2} \mathrm{~m}^{-2} \mathrm{yr}^{-1}\right)$, although, again, I did not attempt to separate OUR from atmospheric exchanges in the surface layers. In the tropical Atlantic, the model's OUR is lower, with $2 \pm 1 \mathrm{~mol} \mathrm{O}_{2} \mathrm{~m}^{-2} \mathrm{yr}^{-1}$ below $\gamma^{n}=26.44$. In the subtropical North Atlantic, OUR is not significantly different from zero beneath $\gamma^{n}=27.16\left(0.3 \pm 0.3 \mathrm{~mol} \mathrm{O}_{2}\right.$ $\left.\mathrm{m}^{-2} \mathrm{yr}^{-1}\right)$ while there is a significant influx above that surface $\left(1.1 \pm 0.2 \mathrm{~mol} \mathrm{O}_{2} \mathrm{~m}^{-2} \mathrm{yr}^{-1}\right)$ (The uncertainty is small compared with the uncertainties of the oxygen transports at $24^{\circ} \mathrm{N}$ and $48^{\circ} \mathrm{N}$ because of the correlations between errors, e.g., page 146). This contrasts 


\begin{tabular}{|l|l|l|l|l|}
\hline Location & Method & OUR & New Prod. & Reference \\
\hline Subtrop. N. Atl. & Tritium budget & 2.4 to 3.5 & & Sarmiento et al. (1990) \\
\hline Subtrop. N. Atl. & ${ }^{228} \mathrm{Ra}$ & $8.5 \pm 0.8$ & & Sarmiento et al. (1990) \\
\hline $54^{\circ} \mathrm{S}-45^{\circ} \mathrm{N}$ & $\begin{array}{l}\text { hydrographic } \\
\text { based model }\end{array}$ & $2.5 \pm 0.8$ & & Riley (1951) \\
\hline Sargasso Sea & Tritium ${ }^{3} \mathrm{He}$ & 7.2 & & Jenkins (1980) \\
\hline $30^{\circ} \mathrm{N}, 30^{\circ} \mathrm{W}$ & He age & $6.4 \pm 0.3$ & & Jenkins (1987) \\
\hline $64^{\circ} \mathrm{N}, 28^{\circ} \mathrm{W}$ & $\begin{array}{l}\mathrm{O}_{2} \text { production } \\
\text { in mixed layer }\end{array}$ & & 5.2 & Peng et al. (1987) \\
\hline near Bermuda & $\mathrm{O}_{2}$ balance & & 3 to 4 & Musgrave (1989) \\
\hline near Bermuda & $\mathrm{O}_{2}$ balance & & $5.6 \pm 1.5$ & Spitzer and Jenkins (1989) \\
\hline
\end{tabular}

Table 4.5: Estimates of oxygen utilization rates and new production $\left(\operatorname{mol~} \mathrm{yr}^{-1} \mathrm{~m}^{-2}\right.$, adapted from Sarmiento et al. (1990).

with the large average OUR of Sarmiento et al. (1990). According to GCM simulations, most of the oxygen input occurs in regions of large oceanic cooling such as the Gulf-Stream north of $36^{\circ} \mathrm{N}$ (e.g., Stephens et al., 1998). The oxygen influx taking place there may dominate the whole signal between $24^{\circ} \mathrm{N}$ and $48^{\circ} \mathrm{N}$. Such a signal is probably underestimated in the Sarmiento et al. (1990) average because of limited data coverage.

In the North Atlantic between $24^{\circ} \mathrm{N}$ and $36^{\circ} \mathrm{N}$, Rintoul and Wunsch (1991) reported a net, "barely significant", loss of oxygen of $1.3 \mathrm{~mol} \mathrm{O}_{2} \mathrm{~m}^{-2} \mathrm{yr}^{-1}$. But again, the gain that we estimate between $24^{\circ} \mathrm{N}$ and $48^{\circ} \mathrm{N}$ may be dominated by the large oxygen influx north of $36^{\circ} \mathrm{N}$, which is not part of the Rintoul and Wunsch (1991) estimate.

Following the work of Watson et al. (1995), Holfort et al. (1999b) estimated the solubility pump as about $1500 \mathrm{kmol} \mathrm{O}_{2} \mathrm{~s}^{-1}$ per PW (Petawatt). The North Atlantic heat loss is, between $24^{\circ} \mathrm{N}$ and $48^{\circ} \mathrm{N}, 0.5 \pm 0.1 \mathrm{PW}$ (Fig. 4-2), which would correspond to an oxygen influx of $750 \pm 150 \mathrm{kmol} \mathrm{O}_{2} \mathrm{~s}^{-1}$. The influx of oxygen diagnosed by the inverse model in the surface layer is in close agreement, at $620 \pm 100 \mathrm{kmol} \mathrm{O}_{2} \mathrm{~s}^{-1}$. There is a similar agreement in the South Atlantic between $30^{\circ} \mathrm{S}$ and $45^{\circ} \mathrm{S}$, with $450 \pm 150 \mathrm{kmol} \mathrm{O}_{2} \mathrm{~s}^{-1}$ predicted from the solubility pump versus $680 \pm 60 \mathrm{kmol} \mathrm{O}_{2} \mathrm{~s}^{-1}$ diagnosed from the model. Globally, however, the solubility effect is significantly smaller than the net observed oxygen sources and sinks, as shown by Fig. 4-11, suggesting that oxygen exchanges driven by biogeochemistry are of major importance in the ocean-roughly $50 \%$.

The Atlantic and Indo-Pacific fluxes are compared with other results in Figure 4-12. 
There is an overall agreement within an uncertainty of one standard deviation, except for the Keeling and Peng (1995) estimate which is directed to the north at the Equator. The agreement in both region between the various estimates, the MIT general circulation off-line model, as well as the Princeton Ocean Biogeochemistry Model is remarkable (and perhaps fortuitous, given the uncertainties).

In conclusion, the estimated oxygen fluxes and flux divergences reflect both solubility and remineralization effects. However, oxygen input through photosynthesis could not be distinguished from other effects in the surface layers because of the large uncertainties in the surface nutrient divergences. Large and significant net oxygen flux divergences are found, with net efflux in the tropical regions and net influx at high latitudes. The global oceanic oxygen budget is in balance. 


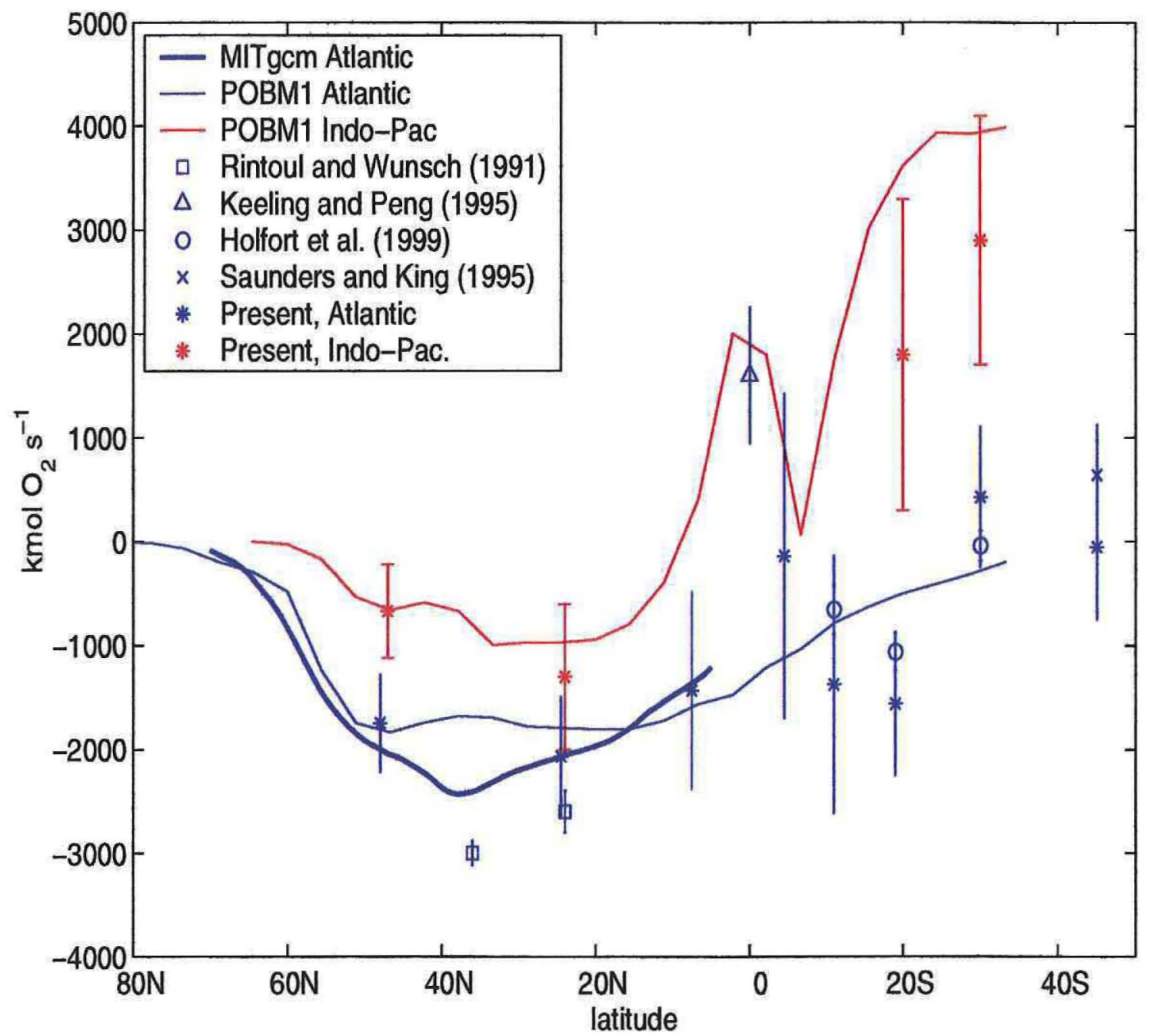

Figure 4-12: Atlantic and Indo-Pacific dissolved oxygen transports (stars). MITgcm=MIT General Circulation Model (offline biogeochemical code; McKinley et al., manuscript in preparation, 1999); POBM1=Princeton Ocean Biogeochemistry Model Version 1 (N. Gruber, personal communication, 1999). 


\section{Chapter 5}

\section{Conclusions}

\subsection{Global summary}

The average oceanic circulation and property transports were estimated over the period 1985-1996, based on the WOCE hydrographic program and the JADE program in the Pacific-Indonesian Throughflow region. Simple dynamics and budget constraints were used to produce an estimate that is consistent, within estimated errors, with hydrographic and current-meter measurements, oceanic dynamics and biogeochemistry.

The physical oceanographic circulation was analyzed with emphasis on the meridional overturning. It is summarized on Figure 5-1. Large scale water mass transports were found to be similar to previous estimates from earlier data in the Atlantic and Indian Oceans. In the Pacific Ocean, differences with previous values were found: a noticeably weaker overturning in the North Pacific Ocean. Deep water is being formed at a rate of $16 \pm 2 \mathrm{~Sv}$ in the North Atlantic, while $20 \pm 6 \mathrm{~Sv}$ of Circumpolar Deep Water is being formed in the Southern Ocean, the latter essentially supplied by the former. Through the use of anomaly equations, significant dianeutral diffusivities associated with tracer transports were found in the deep layers of most oceanic regions, averaging globally $3 \pm 1 \mathrm{~cm}^{2} \mathrm{~s}^{-1}$ in the 2000-4000 dbars depth range, excluding the Southern Ocean. In the Southern Ocean, significant diffusivities could not be determined because of the large uncertainties in layers that are in contact with the surface.

The freshwater flux divergences are $100 \%$ uncertain in most places, but there is an 


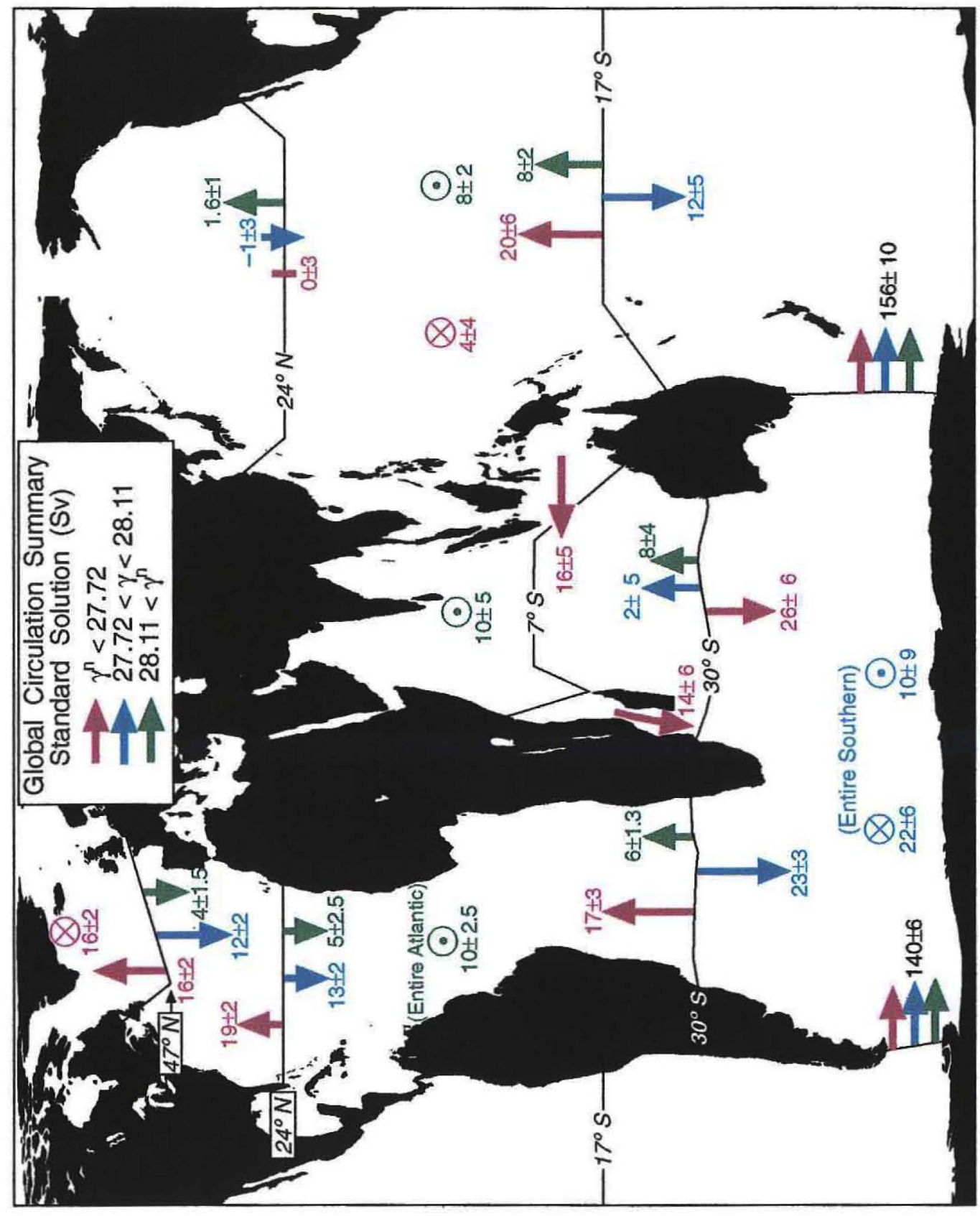

Figure 5-1: Global circulation summary. The water transports are indicated (approximately) for the different density classes. The exact boundaries between surface, deep and bottom waters are $\gamma^{n}=27.82 / 28.0986$ (North Atlantic); 27.72/28.11 (South Atlantic); 27.7/28.11 (Indian); 27.8/28.10 (Pacific). The color of the upwelling arrows indicate the layer from which the water is coming. In the Southern Ocean, while the bottom water formation takes place mostly in the Weddell Sea, upwelling is uncertain and spread over the different regions. The South Pacific transports are given at $17^{\circ} \mathrm{S}$ because of the more complicated structure at $32^{\circ} \mathrm{S}$ (Fig. 3-7). 
overall agreement with previous estimates. Energy transports evidence significant oceanatmosphere heat fluxes, with a global heating of $2.3 \pm 0.4 \mathrm{PW}$ in the tropical band. Most of the cooling occurs in the Northern Hemisphere $(1.7 \pm 0.2 \mathrm{PW})$ while in the Southern Hemisphere only $0.7 \pm 0.3 \mathrm{PW}$ is transferred to the atmosphere.

Silica, nitrate and phosphate fluxes and divergences were analyzed with respect to their respective biogeochemical cycles. Determination of nutrient divergences appears to be at the edge of the model and data capabilities, but despite the large uncertainties, the magnitude of the maximum export production agrees closely with independent measurements from moorings and isotopic data. The (possible) signature of a large-scale average export production is found for silica, nitrate and phosphate in several temperate regions. The nutrient divergences thus provide an upper bound on the average remineralization rate in several depth ranges while the lower bound is more uncertain. Net nutrient sources or sinks are found in several regions. Such divergences suggest advection of dissolved organic matter across the sections, as first pointed out by Rintoul and Wunsch (1991), unless those are spurious as a result of aliasing the seasonal cycle.

Significant exchanges of oxygen with the atmosphere are found, with oxygen intake at high latitudes and loss at low latitudes. About half of the oxygen gain in the surface layer can be explained by changes in solubility through temperature changes, leaving an important role to the biology-driven exchanges. Indeed, significant oxygen utilization is found below the surface in several regions, indicating remineralization.

\subsection{Discussion and perspectives}

Exchanges of carbon dioxide between ocean and atmosphere are related to the oxygen exchanges. While the solubility pump produces influxes and effluxes of $\mathrm{CO}_{2}$ that are similar to $\mathrm{O}_{2}$, the biological pump creates exchanges in the opposite direction. With the oxygen budget being in balance over the whole ocean, there is a suggestion that the solubility and biological $\mathrm{CO}_{2}$ exchanges are in balance as well-at least within uncertainties.

On the other hand, oceanic uptake of anthropogenic $\mathrm{CO}_{2}$ may occur as a simple partial pressure equilibration, independently from the biological and solubility pumps, as has been 
suggested by several authors (e.g., Sarmiento et al., 1992).

Combination of ocean dynamics with geochemical cycles represents one more step towards producing a consistent estimate of the oceanic circulation. Use of a simple timeaverage model permits estimation of meridional property fluxes based on the highest resolution allowed by hydrographic data. Such a model permits estimation of the true uncertainties of the property fluxes. The physical circulation estimated here during the WOCE period provides a reference for climate studies, for instance as a way to test general circulation models. The estimated ocean-atmosphere heat and freshwater transfers can be used, similarly, as a benchmark for climatology, while average dianeutral diffusivities will help in parameterizing the sub-grid-scale processes in numerical models.

I chose a standard, or preferred, solution among a large number of experiments (about 100 in total), but there is no limit to possible configurations of the model and detailed regional studies will improve the model consistency.

But a time-average budget relies upon the ergodic hypothesis, and the remaining uncertainty is dominated by oceanic variability in the density and other properties. The uncertainties presented here account for the errors from both the model limitations and the measurements. In particular, the alias errors in the mass transports resulting from non-synopticity of the sections and oceanic variability is taken into account by reference to a general circulation model simulation.

The analysis of the present uncertainties and model limitations suggests several directions for future work. The necessary next step includes the use of additional data, some of which may already exist (e.g., current-meters, floats, absolute altimetric data) combined with more complete dynamics to eventually reduce the residual uncertainty in the solutions. In particular, the property fluxes through the Pacific-Indonesian Throughflow (PIT) and other highly variable currents such as the North Brazil Current are a large source of uncertainty, due to the lack of measurements and the large seasonality there.

\subsubsection{New data}

Introduction of new data can be simulated in order to optimize their impact on the reduction of the existing uncertainties. Naturally, new high-quality surveys will increase the 
resolution of the flux divergence estimates. But if the scope is to estimate, for instance, air-sea heat exchanges or export production, such surveys could be designed in order to optimize the signal, based on existing maps of heat exchanges from climatologies or atmospheric models. The present surveys are mostly zonal or meridional, and several "boxes" contain regions of strong heating and regions or strong cooling that mostly cancel out. As a result, the inverse model diagnoses only the small residual air-sea exchange.

Inclusion of the existing meridional hydrographic sections in the present model will be a considerable challenge. Such work is in progress in several oceanic areas. The major obstacle to combining zonal and meridional sections is the treatment of crossing points, which can lead to large mass imbalances in individual layers due to temporal changes in the eddy field from one section to the next (i.e., Wunsch and Grant, 1982). The a priori noise in the mass flux has to be modified accordingly.

\subsubsection{Improvements to the present model}

The present circulation estimate will improve with time as it benefits from suggestions for improvement on the different parameter choice such as the initial reference surface based on new current meter measurements and water mass analysis where the signals are important such as in the boundary current regions. The experiments that I did (e.g., in the Indian Ocean) suggested that large scale mass transports are relatively insensitive to such changes, although there is no guarantee that the same is true everywhere.

The parameterization of dianeutral exchanges as a single diffusion and advection coefficients over large oceanic areas may be unrealistic. In particular, the possibility of double diffusion was not addressed, and use of a different coefficient of heat and tracer transfers may be used in future versions of the model. Dianeutral exchanges are also poorly parameterized in regions of outcrop such as the Southern Ocean. There, an advection-diffusion scheme is probably unrealistic and an alternative parameterization may be to assign an independent transfer coefficient to each property (e.g., Sloyan and Rintoul, 1999a,b), but requiring extra data to resolve them separately.

The calculation of Ekman property transports may be improved as well by, for instance, estimating the transport across each station pair and explicitly using the vertical property 
distribution (e.g., Wijffels et al., 1999).

Lastly, the a priori statistics on the model can be improved. For instance, the weight of anomaly equations can be directly calculated from a simulation by a general circulation model as I did for the mass fluxes, instead of the ad-hoc estimate that was used here. Variability in the nutrient fluxes is another major possible alias that could not be quantified. Seasonal correlations between changes in the baroclinic transports and nutrient concentration in the upper layers can create such aliases in the nutrient transport computed from a single hydrographic survey. Repeat survey will provide useful insights for such variability, would the measurement be made only over the upper layers that are the most sensitive to seasonal cycle.

\subsubsection{Improving the dynamics}

Including complete dynamics is the ultimate target of ocean estimation. In this model, variability in the density field is a major source of uncertainty and limits the impact of new data. Allowing a correction to the density field permits achievement of better mass conservation and optimizes the impact of new data. Simple steady-state (or time-average) models with corrections to the density field allowed through the inversion have been used in the past (e.g., Wunsch, 1994). But the major obstacle, apart from computational load, is the lack of knowledge about a priori statistics for the density variability. Global spectra of the density field variability may partially solve the problem (Zang, 1999), although the variability has a strong spatial dependence.

Data assimilation in general circulation models is the natural next step toward ocean circulation estimation. Computer capabilities are currently limiting the resolution and prohibiting calculation of uncertainties. Assimilation of data such as hydrographic sections remains a considerable challenge, but the ongoing efforts, in parallel with simple statistical estimates such as the one presented here, will keep improving our estimate of the timevarying ocean circulation. 


\section{Appendix A}

\section{Ageostrophy in the General Circulation Model}

While geostrophy is generally a reliable approximation in the ocean, the Semtner and Chervin model output contains substantial ageostrophic transports. Their cause and nature are investigated in this Appendix.

The ageostrophic component at $36^{\circ} \mathrm{N}$ was computed as the difference $v_{\text {ageost }}=v_{\text {model }}-$ $v_{g a}$, where $v_{g a}$ is the absolute geostrophic velocity defined by (2.8). The GCM uses a staggered B grid (e.g., Semtner, 1986; Killworth et al., 1991), so that $v_{g a}$ is staggered with respect to temperature, $T$, salinity, $S$ and sea surface elevation, $\eta$ (Figure A-1). In principle, $v_{g a}$ can be compared with the model velocity, $v_{m}$, on the original $u, v$ grid. Equivalently, the comparison was done on an intermediate grid, called the sample grid (superscripts $s$ ) on to which all the state variables are linearly interpolated. Namely, the model $u, v$, noted $\left(u^{m}, v^{m}\right)$, were interpolated at the temperature points in the East-West direction, yielding the sample field $\left(u^{s}, v^{s}\right)$ while $T, S$ and $\eta$ were interpolated in the North-South direction, so that all data stand on the $s$ grid (Fig. A-1a). At topography, the geostrophic velocity was assumed to be zero before interpolation. This corresponds to the no-slip boundary condition for model velocity.

The ageostrophic velocity was then computed at each "sample" grid point 

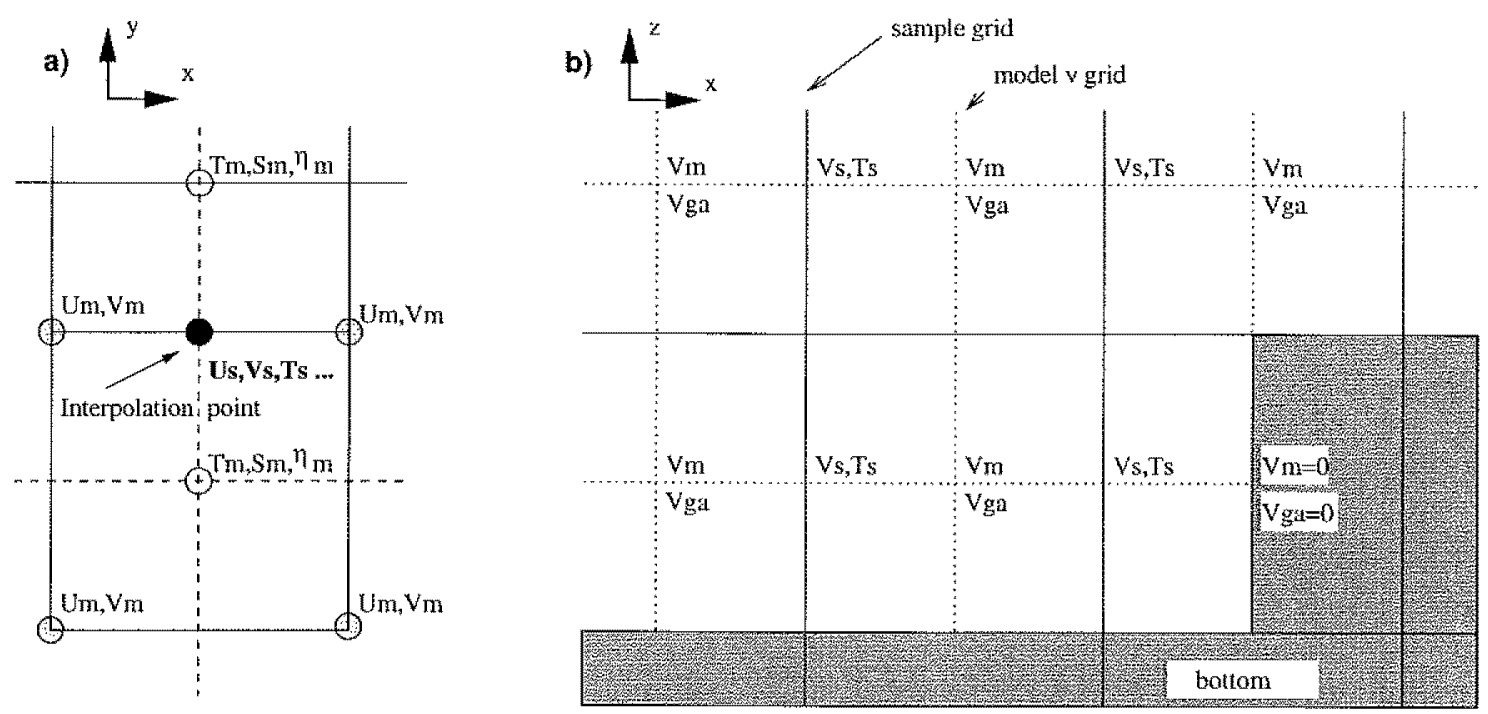

Figure A-1: Model B grid and sampling for a zonal section. Subscript $m$ stands for the original model $(u, v)$ grid; subscript $s$ for the points at which the fields are interpolated ("sampled"), and $g$ for geostrophic. $T, S, \eta$ are interpolated in the North-South direction. a) top view; b) vertical and zonal section.

(superscript s):

$$
v_{\text {ageost }}^{s}=v^{s}-v_{g a}^{s} \text {. }
$$

Locally, the ageostrophic velocities are noisy and difficult to interpret, and zonal or vertical integrations are helpful for determining the transports structure. Figure A-2 shows a snapshot of the cumulative ageostrophic transport through each vertical grid interval, integrated from the eastern end at $36^{\circ} \mathrm{N}$, revealing strong variations of $\pm 5 \mathrm{~Sv}$ (1 Sverdrup $=10^{6}$ $\left.\mathrm{m}^{3} \mathrm{~s}^{-1}\right)$ at the western end.

A frequency spectrum (not shown) of the net ageostrophic transport below the Ekman layer shows a very large amount of energy at high frequencies. It turns out that most of the ageostrophic transport is due to inertial and possibly internal waves.

Because the GCM output was subsampled only every third day, inertial waves are aliased into frequencies that vary with latitude, as discussed in Jayne and Tokmakian (1997). A 15-day low pass filter, of the form $\frac{1}{15} \operatorname{sinc}\left(\frac{\tau}{15}\right)$ (where $\tau$ is the time lag) was applied to all the state variables of the GCM output to filter out high frequencies including the inertial waves. (At $36^{\circ} \mathrm{N}$, the frequency of an inertial oscillation is $s_{0}=1.176$ day $^{-1}$ so 


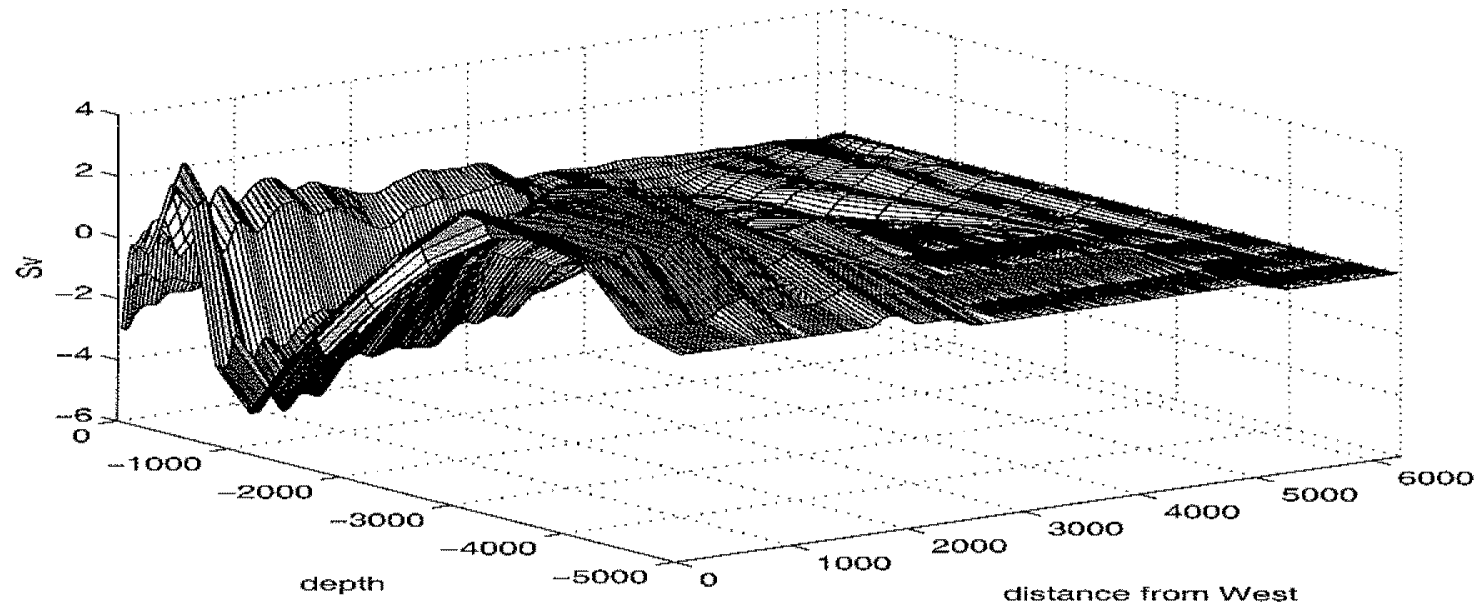

Figure A-2: Cumulative ageostrophic transport in each layer of the model, integrated from the eastern end, section $\mathrm{A} 36^{\circ} \mathrm{N}$.

that the 3-day alias frequency is $s_{\text {alias }}=(6.34 \text { day })^{-1}$.) Figure A-3 shows the integrated ageostrophic transport in a snapshot of the filtered field: it is now dominated by the Ekman transport. In the interior and away from the western boundary current, ageostrophic transport accumulates up to at most a few tenths of a Sverdrup in each layer. Near the western boundary current (WBC) region, the integrated value increases up to $\pm 1 \mathrm{~Sv}$.

The low-pass filtered, vertically-integrated absolute and geostrophic transports through each grid cell are displayed on Figure A-4a for one time period. Below the Ekman layer, the ageostrophy is of less than $1 \mathrm{~Sv}$ (Fig. A-4b, dotted lines), showing that the model was close to geostrophic equilibrium in the interior. However, near the Gulf-Stream the departure attains $5 \mathrm{~Sv}$ per $36 \mathrm{~km}$ grid cell, which is up to $50 \%$ of the total transport in the cell. In this ageostrophic region, the model velocity transports are weaker than the geostrophic ones (Fig. A-4a).

When integrated from the East (Fig. A-4c), the cumulated ageostrophic transport grows up to $5 \mathrm{~Sv}$, and is more or less cancelled in the WBC region, to yield a, total ageostrophic flux of $2 \mathrm{~Sv}$ below the top $100 \mathrm{~m}$. Nevertheless, this cancellation is accidental to this snapshot and systematic deviations of up to $5 \mathrm{~Sv}$ are observed persisting for a few weeks. There is no apparent correlation between the ageostrophy and the topographic 


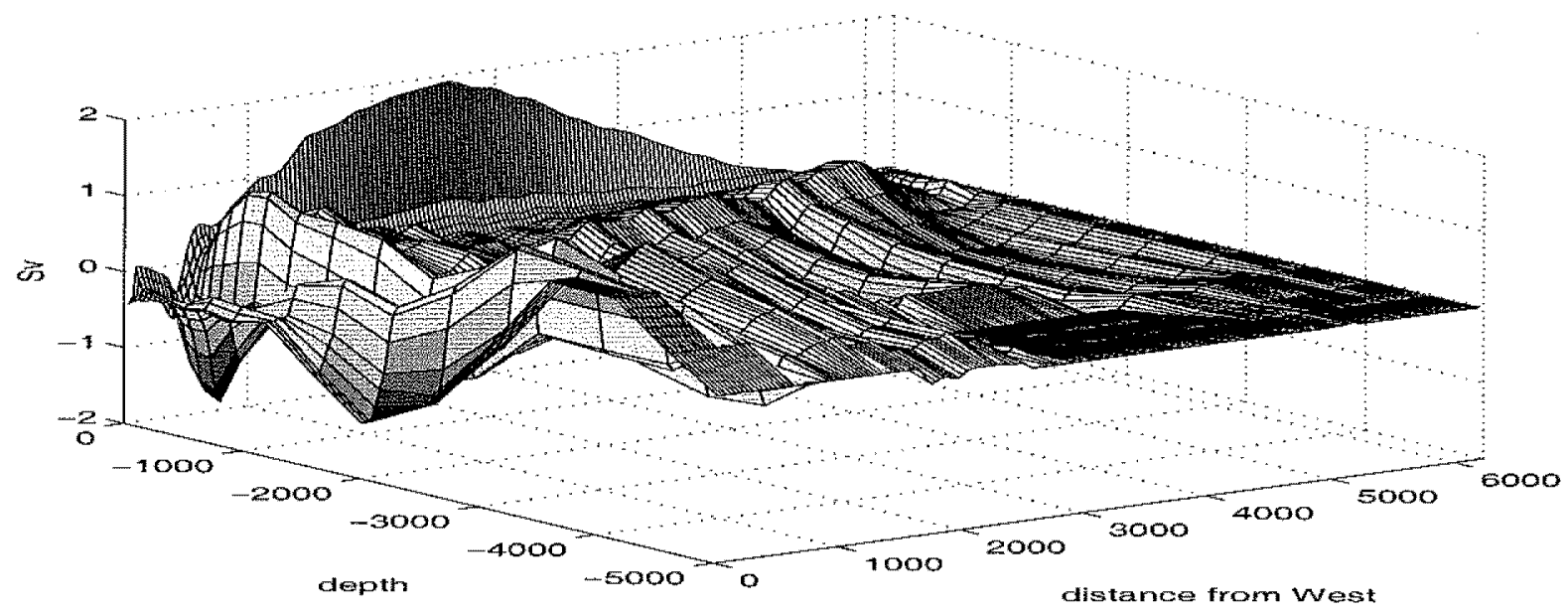

Figure A-3: Same as previous figure, but with inertial oscillations filtered out.

features (Fig. A-4d).

What process is responsible for a 50\% deviation from geostrophy? The GCM equation for $u$ is

$$
\frac{\partial u}{\partial t}+u \frac{\partial u}{\partial x}+v \frac{\partial u}{\partial y}-f v=-g \frac{\partial \eta}{\partial x}+\nu \nabla^{4} u
$$

At the surface, the Rossby number (based on the $36 \mathrm{~km}$ grid-step) is

$$
\epsilon=\frac{U}{f L}=O\left(\frac{0.5}{10^{-4} \times 36 k m}\right) \simeq 0.15
$$

A direct calculation of $\left(u \frac{\partial u}{\partial x}\right) /(f v)$ from the model velocity and horizontal shear produces a similar value. The non-linear term is not able to explain the ageostrophic transport. If the Gulf-Stream curvature occurs on similar scales, the cyclostrophic term is of the same order as the non-linear terms, and is thus small in the model.

The horizontal hyperviscosity coefficient in the model is about $10^{12} \mathrm{~m}^{4} \mathrm{~s}^{-1}$ (Stammer et al., 1996) so that the horizontal Ekman number ([viscous term]/[geostrophic term]) is of order:

$$
\frac{\nu \nabla^{4} u}{f v} \sim \frac{\nu}{f L^{4}}=\frac{10^{12}}{10^{-4} \times(36 \mathrm{~km})^{4}} \simeq 6 \times 10^{-3}
$$



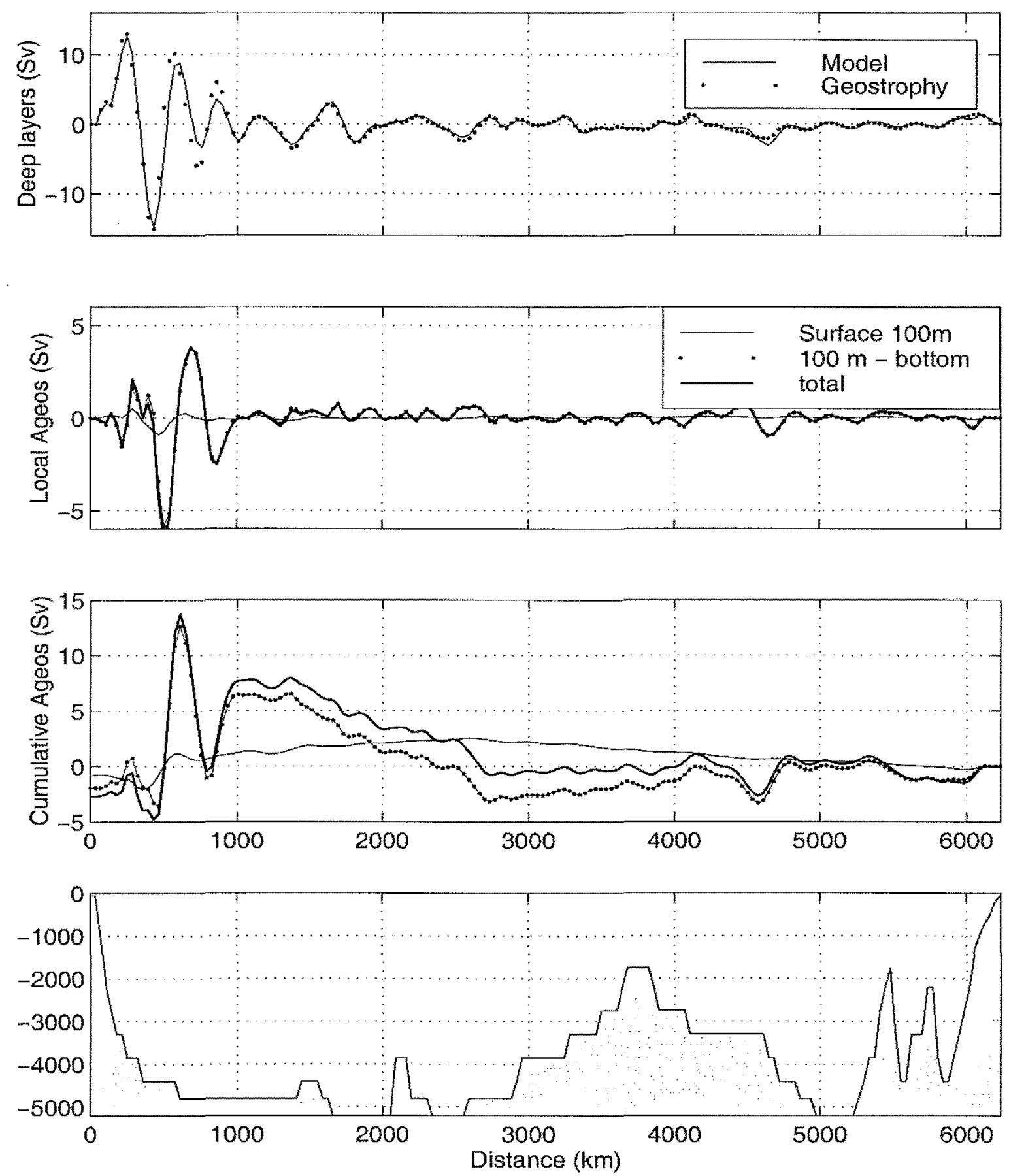

Figure A-4: Vertically integrated transport per grid interval from the a) model absolute velocity and geostrophic velocity, from $100 \mathrm{~m}$ to bottom; b) ageostrophic velocity and c) ageostrophic velocity integrated from East. d) GCM bottom topography. Inertial waves were removed by filtering. 
which is too small to explain the observed ageostrophy.

The approximations of the geostrophic calculation near a sloping bottom (which is a stair-case in the model) is also unlikely to explain the ageostrophy as the bottom topography did not show any correlation with the ageostrophic transport.

An examination of the vertical structure of the velocity in the Gulf Stream shows that the GCM shear is close to the thermal wind, with the ageostrophic transport being essentially depth-independent. (The thermal wind is referenced to the surface, and the geostrophic surface velocity calculated from $\frac{\partial \eta}{\partial x}$ is used to produce the geostrophic transport.) For a $36 \mathrm{~km}$ grid cell, a deviation of $3 \mathrm{~cm} \mathrm{~s}^{-1}$ over the water column $(5000 \mathrm{~m})$ produces the $5 \mathrm{~Sv}$ observed deviation. However, this deviation is not justified by any of the terms in the momentum equations.

The strong ageostrophy observed in GCM transport is most likely due to numerical problems. One possibility is the presence of an enhanced bottom friction in the barotropic equation of the GCM. In principle, the exact source of ageostrophy is identifiable by examining the size of each term in the original numerical equations. This requires a more complete GCM extraction than the one we have and is beyond the present work. In any case, the depth-independent nature of the ageostrophy makes us confident in using the GCM output to estimate baroclinic variability.

An alternative explanation is purely physical: in the presence of a strong current, the Ekman flux is enhanced through the non-linear interaction with the wind stress. The sea surface elevation may be slightly shifted from the elevation due to the underlying geostrophic currents (e.g. Niiler, 1969). In the GCM, there is no explicit mixed layer, but the diffusivity is dependent on the Richardson number so that one can have a strong mixing is the first layers. Estimating the amplitude of this effect is beyond the scope of the present work. 


\section{Appendix B}

\section{Model Error in a Two-dimensional}

\section{Ocean}

A hypothetical two-layer, two-dimensional ocean is used to illustrate the effect of the model error on the representativeness of the heat and silica flux calculation. The "true" circulation is sketched in Figure B-1a: the basin is closed to the north of the section so that it is known that net mass losses or sources do not exceed the freshwater flux, known to an accuracy of $\pm 0.5 \mathrm{~Sv}$. The true circulation is a $20 \mathrm{~Sv}$ overturning. The upper layer is $15^{\circ}$ water with a silica concentration of $10 \mu \mathrm{mol} \mathrm{Si} / \mathrm{kg}$ while the lower layer is $2^{\circ}, 30 \mu \mathrm{mol} \mathrm{Si} / \mathrm{kg}$. The actual heat flux divergence is given by

$$
\begin{aligned}
H_{\text {true }} & =\int \rho C_{p} v \theta d x d z \\
& \simeq 1000 \times 4000 \times 20 \times 10^{6} \times(15-2) \mathrm{kg} \mathrm{m}^{-3} \mathrm{Jkg}^{-1} \mathrm{~K}^{-1} \mathrm{~m}^{3} \mathrm{~s}^{-1} \mathrm{~K} \\
& \simeq 1 \mathrm{PW}
\end{aligned}
$$

and the silica divergence is:

$$
\begin{aligned}
S_{i, \text { true }} & =\int \rho v C d x d z \\
& =1000 \times 20 \times 10^{6} \times(10-30) \times 10^{-6} \\
& =-400 \mathrm{kmol} \mathrm{S}_{i} \mathrm{~s}^{-1} .
\end{aligned}
$$




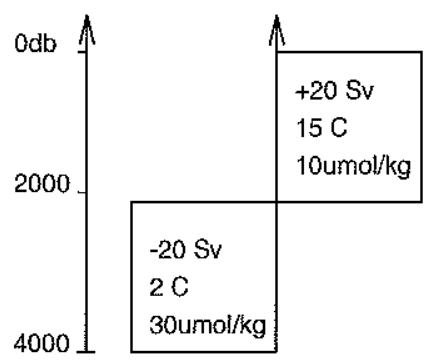

a) true

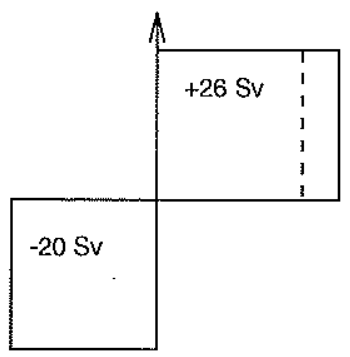

b) observed

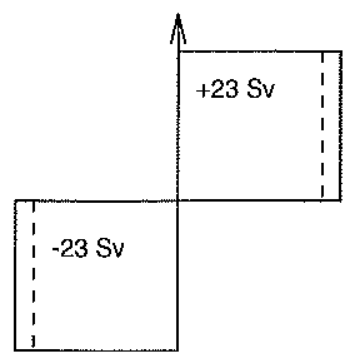

c) inversion

Figure B-1: Two-dimensional overturning example model. In the true, or time-average case (a) the upper layer carries $20 \mathrm{~Sv}$ northward of $15^{\circ} \mathrm{C}$ water with a silica concentration of $10 \mu \mathrm{mol} \mathrm{Si} \mathrm{kg}{ }^{-1}$ while the lower layer carries $20 \mathrm{~Sv}$ of $2^{\circ} \mathrm{C}, 30 \mu \mathrm{mol} \mathrm{Si} \mathrm{kg}{ }^{-1}$ water southward. Because of the baroclinic variability, a mass excess of $6 \mathrm{~Sv}$ is observed in the upper layer (b), leading to the mass-conserving circulation (c).

(The silica flux is a unrealistic in this example as it should be small in the real ocean, which is not possible in this 2-D model.) The goal is to recover this solution using an inverse model requiring mass conservation. Because the model is two-dimensional, the barotropic correction to mass imbalances produces an adjustment in the property fluxes of $\Delta_{M} \times\left(C_{1}+C_{2}\right) / 2$, where $C_{1}$ and $C_{2}$ are the concentrations in the upper and lower layers, and $\Delta_{M}$ is the adjustment to the total mass flux.

\section{1) no measurement errors}

First, suppose that there were no errors in the measurements, (no baroclinic variability, no internal waves) and that we picked a reference level that is equal to $2000 \mathrm{dbars}$ and that exactly reproduces the true circulation. Then, there is no initial mass imbalance and the inverse model produces the true solution. The derived heat and silica flux are the true ones. An a priori uncertainty in $\Delta_{M}$ of $\delta_{M}= \pm 1 \mathrm{~Sv}$ in the zero-mass-flux equation implies an uncertainty on the property fluxes of

$$
\begin{aligned}
\delta H_{ \pm 1} & =\rho C_{p} \delta_{M}\left(T_{1}+T_{2}\right) / 2 \\
& =1000 \times 4000 \times 10^{6} \times(15+2) / 2= \pm 0.034 P W \\
\delta S i_{ \pm 1} & =10^{6} \times(10+30) / 2 \times 10^{-6}= \pm 20 \mathrm{kmol} \mathrm{S}_{i} \mathrm{~s}^{-1}
\end{aligned}
$$




\section{2) baroclinic error of $5 \mathrm{~Sv}$}

Now, suppose that for various reasons the measured/calculated shear is off by $6 \mathrm{~Sv}$ with respect to the time average circulation, or the "true" circulation (Figure B-1b). With the mass conservation constraint alone, the inverse model adds $3 \mathrm{~Sv}$ to each layer, producing the circulation on Figure B-1c. The resulting fluxes are:

$$
\begin{aligned}
H_{i n v} & =4 \times 23 \times 10^{12} \times(15-2)=1.2 P W \\
S_{i, i n v} & =23 \times(10-30)=-460 \mathrm{kmol} \mathrm{S}_{i} \mathrm{~s}^{-1}
\end{aligned}
$$

If one would have constrained the circulation to conserve mass within $\pm 1 \mathrm{~Sv}$, the fluxes would be

$$
\begin{aligned}
H_{i n v} & =1.2 \pm 0.034 P W \\
S_{i, i n v} & =-460 \pm 20 \mathrm{kmol} \mathrm{S}_{i} \mathrm{~s}^{-1}
\end{aligned}
$$

which is clearly incompatible with the true solution (B.1-B.2). On the other hand, an a priori uncertainty of $\pm 10 \mathrm{~Sv}$ in the mass equation, would yield

$$
\begin{aligned}
H_{i n v} & =1.2 \pm 0.34 P W \\
S_{i, i n v} & =-460 \pm 200 \mathrm{kmol} \mathrm{S}_{i} \mathrm{~s}^{-1}
\end{aligned}
$$

which is realistic as the model error is properly taken into account.

If the model is not able to conserve mass properly because of other constraints so that the initial mass imbalance of $6 \mathrm{~Sv}$ remains, the solution is:

$$
\begin{aligned}
H_{i n v} & =4 \times 10^{12} \times(26 \times 15-20 \times 2) \\
H_{i n v} & =1.4 \pm 0.34 P W \\
S_{i, i n v} & =10 \times 26-30 \times 20 \\
S_{i, i n v} & =-340 \pm 200 \mathrm{kmol} \mathrm{S}_{i} \mathrm{~s}^{-1}
\end{aligned}
$$

which is consistent with the true solution ( $1 \mathrm{PW},-400 \mathrm{kmol} \mathrm{Si} \mathrm{s}^{-1}$ ). (The uncertainties correspond to one standard deviation.) 


\section{3) anomaly formulation}

In this particular case, the anomaly formulation yields the exact answer:

$$
\begin{aligned}
H_{i n v} & =1.4-4 \times 10^{12} \times(15 \times 6) \\
H_{i n v} & =1 \pm 0.26 P W \\
S_{i, i n v} & =-360-6 \times 10 \\
S_{i, i n v} & =-400 \pm 100 \mathrm{kmol} \mathrm{S}_{i} \mathrm{~s}^{-1}
\end{aligned}
$$

Note the importance of using the average property concentration in the layers where the mass residual occurs rather than having a global average property concentration. 


\section{Appendix $\mathbf{C}$}

\section{A priori mass uncertainties}

The first table gives the basic a priori uncertainty contribution from each section. The second table gives the a priori uncertainty used in each box. (For comparison, the uncertainty including the Ekman transport uncertainty are indicated in the shaded rows. Because an adjustment to the Ekman transport is permitted, those are not used in the inverse model.) 


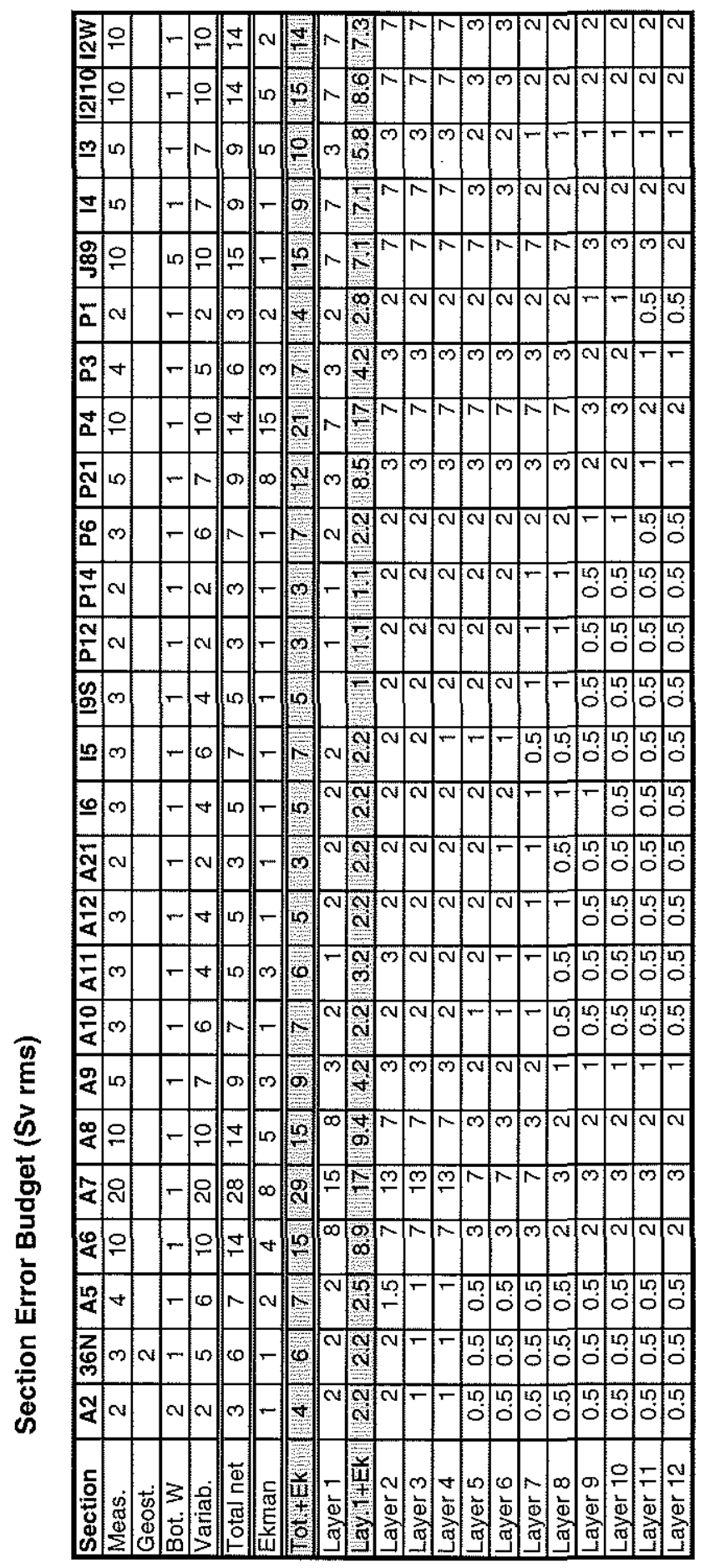

Figure C-1: A priori uncertainty contribution from each section, in Sv rms. The "total net" (5th row) indicates the total uncertainty in the net flux. 


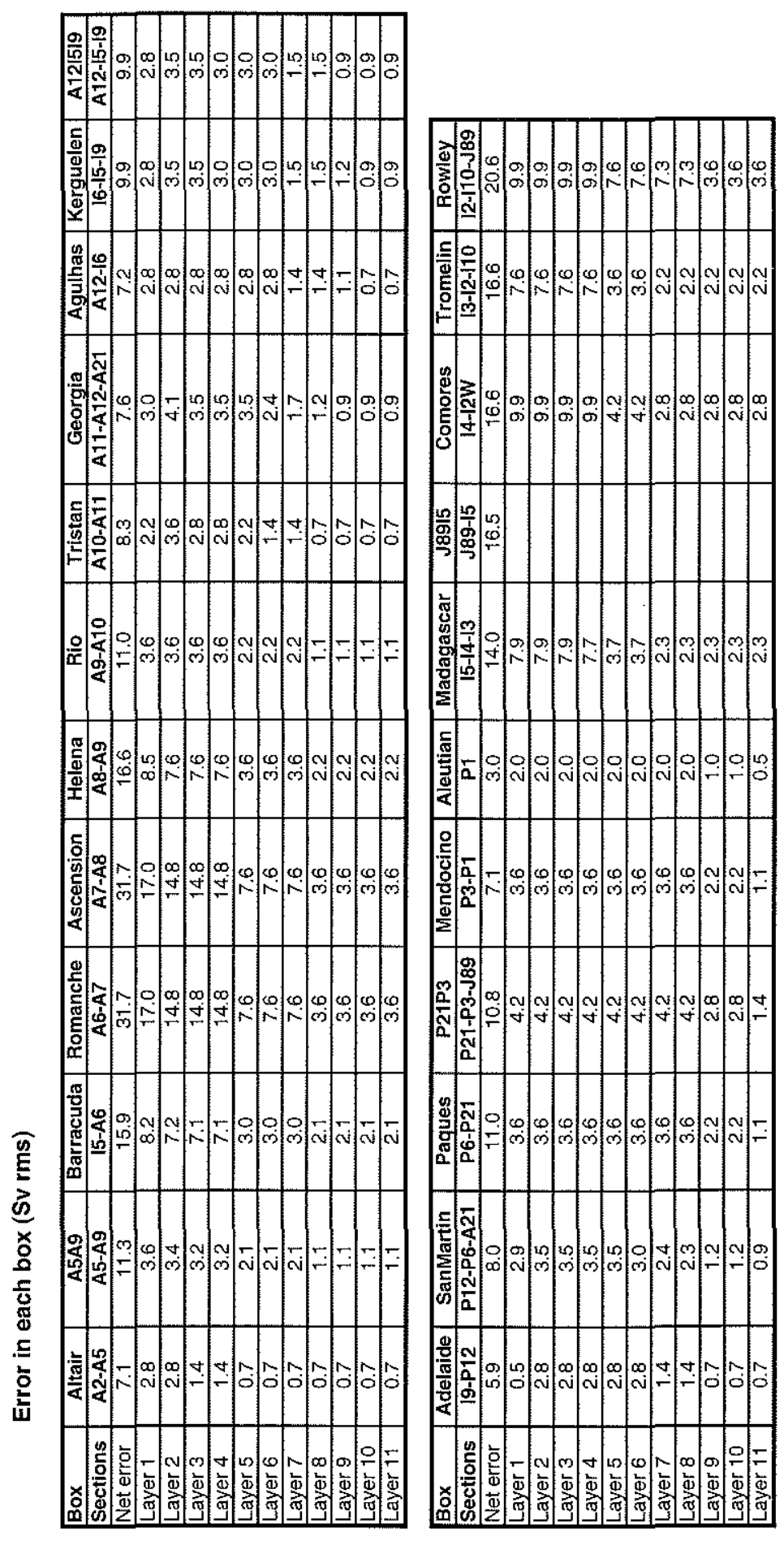

Figure C-2: A priori uncertainty on each mass conservation constraint. 


\section{Appendix D}

\section{Standard solution absolute velocities and cumulative \\ transports}

For each section:

(i) The first figure shows the absolute velocity field across the section. The initial reference surface is indicated by the dash-dot line on the absolute velocity plots. The velocities are positive northward and eastward. Negative velocity regions are shaded. The contour intervals are $1 \mathrm{~cm} \mathrm{~s}^{-1}$ (thin contours, from $-10 \mathrm{~cm} \mathrm{~s}^{-1}$ to $10 \mathrm{~cm} \mathrm{~s}^{-1}$ ) and 10 $\mathrm{cm} \mathrm{s}^{-1}$ (thick contours for stronger flows). The reference velocity (or velocity on the reference surface after integration) is given in the panel above the velocity contour plots, the shaded area giving the uncertainty (one standard deviation).

(ii) The second figure gives the transports integrated over several layers and between the neutral surfaces that are indicated-those roughly correspond to the water masses defined in the text. The thick plain line gives the absolute transport; the dashed line gives the contribution from the thermal wind; and the dots give the contribution from the reference velocity adjustment. The shaded areas give the uncertainty on the absolute transport (one standard deviation). The Ekman transport is not included. 


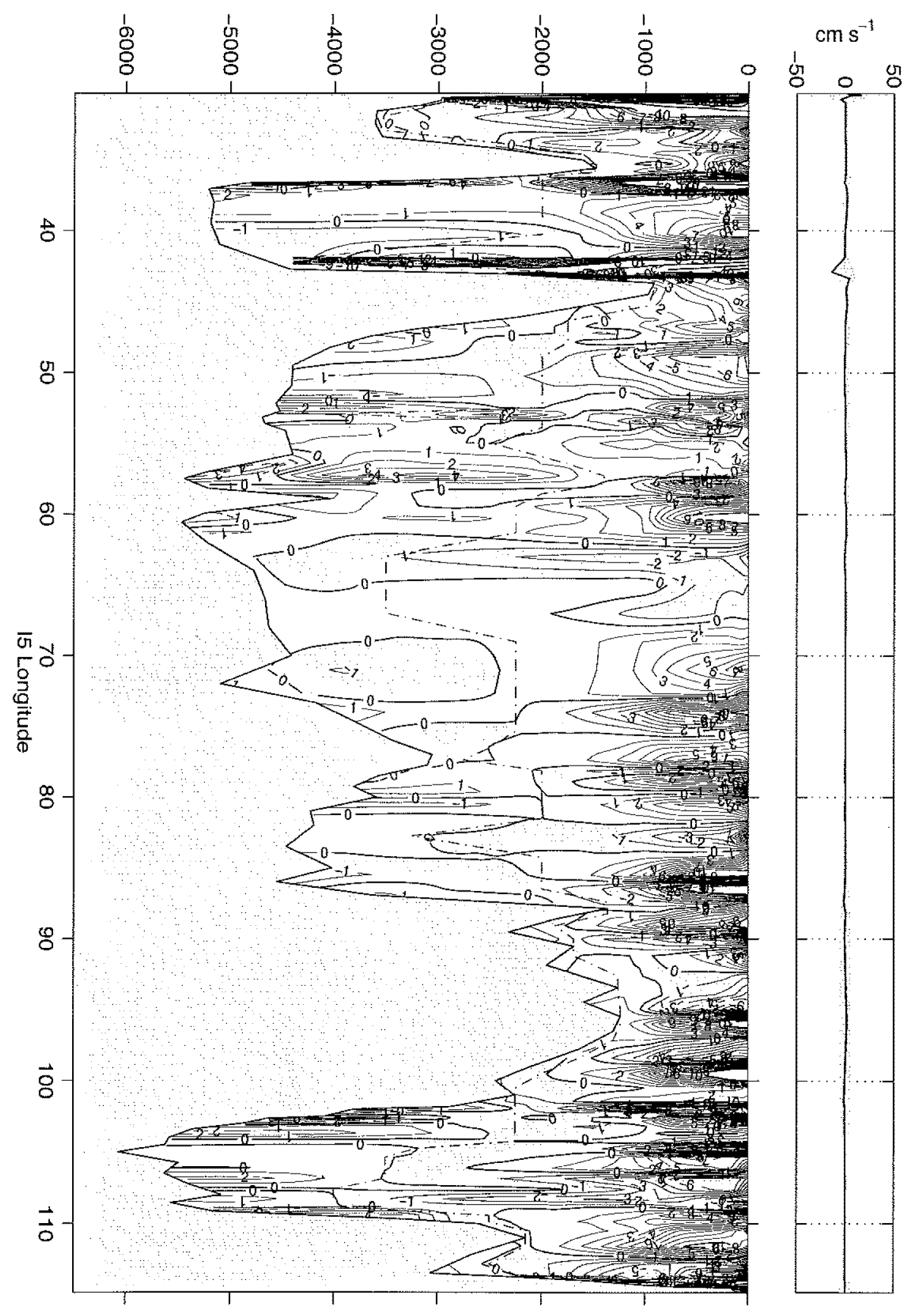

Figure D-1: Absolute velocities, Indian Ocean, I5 (32 $\mathrm{S})$ 

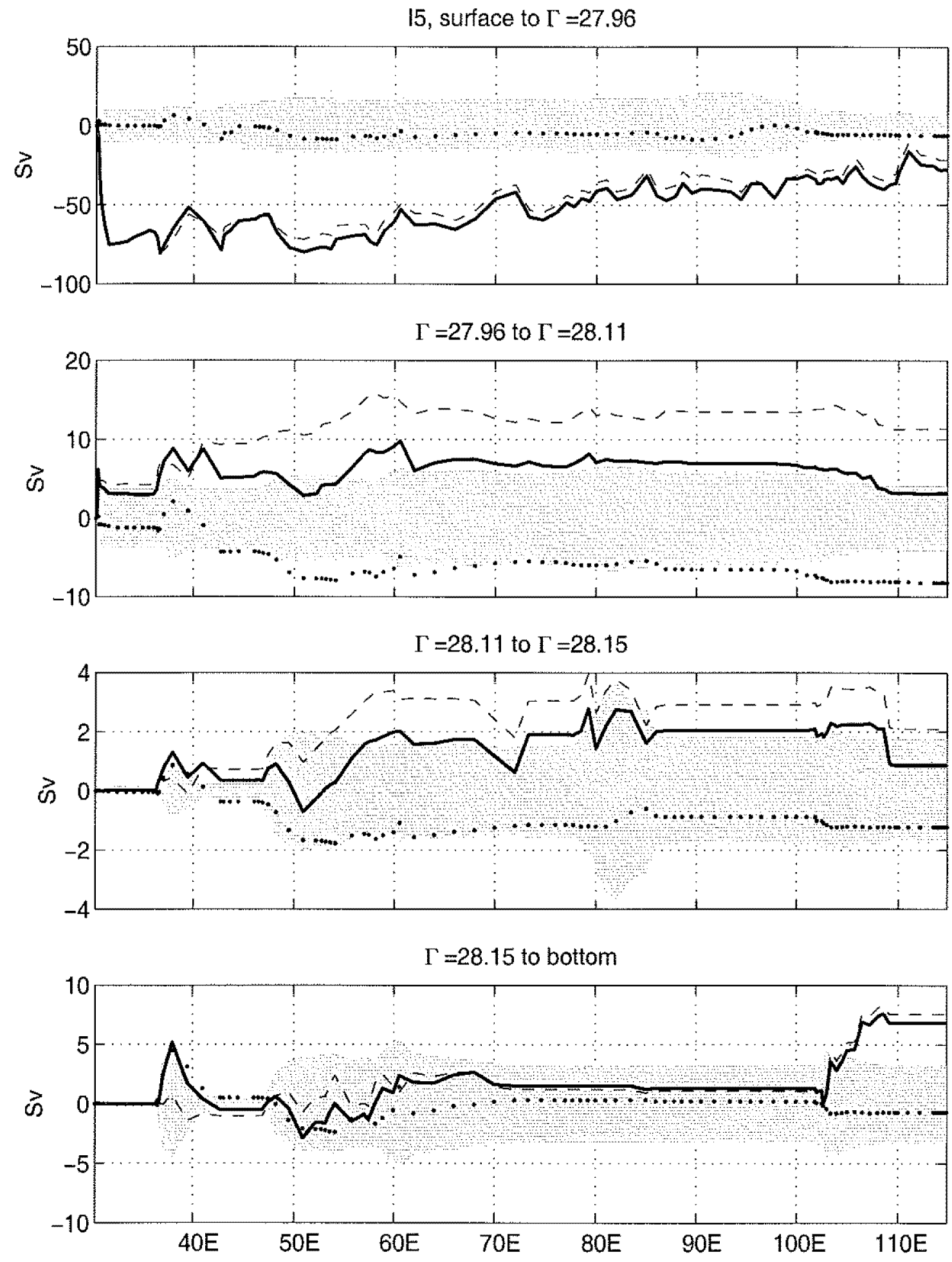

Figure D-2: Cumulative transports, Indian Ocean, I5 $\left(32^{\circ} \mathrm{S}\right)$ 


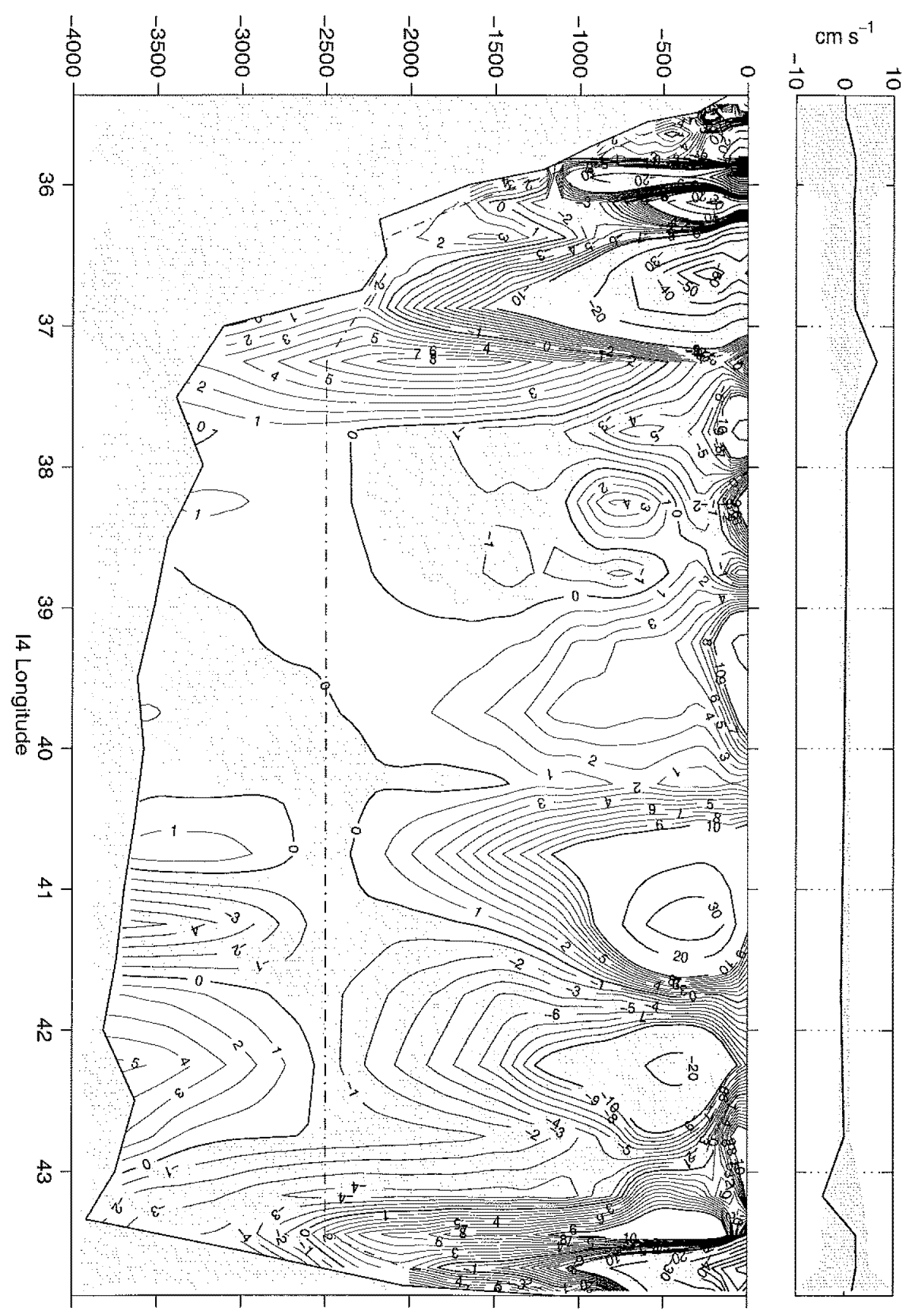

Figure D-3: Absolute velocities, Indian Ocean, $14\left(25^{\circ} \mathrm{S}\right)$ 

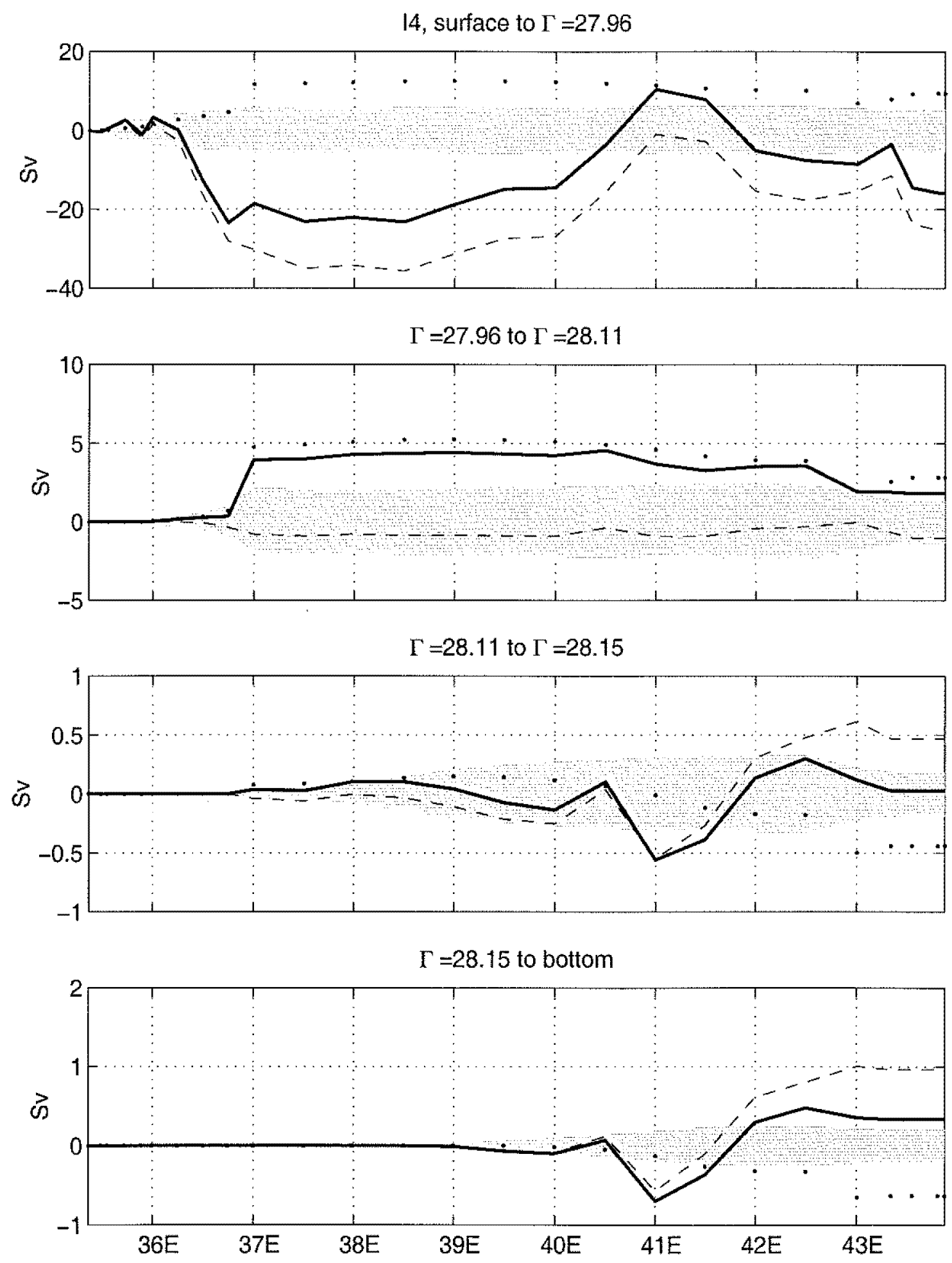

Figure D-4: Cumulative transports, Indian Ocean, $14\left(25^{\circ} \mathrm{S}\right)$ 


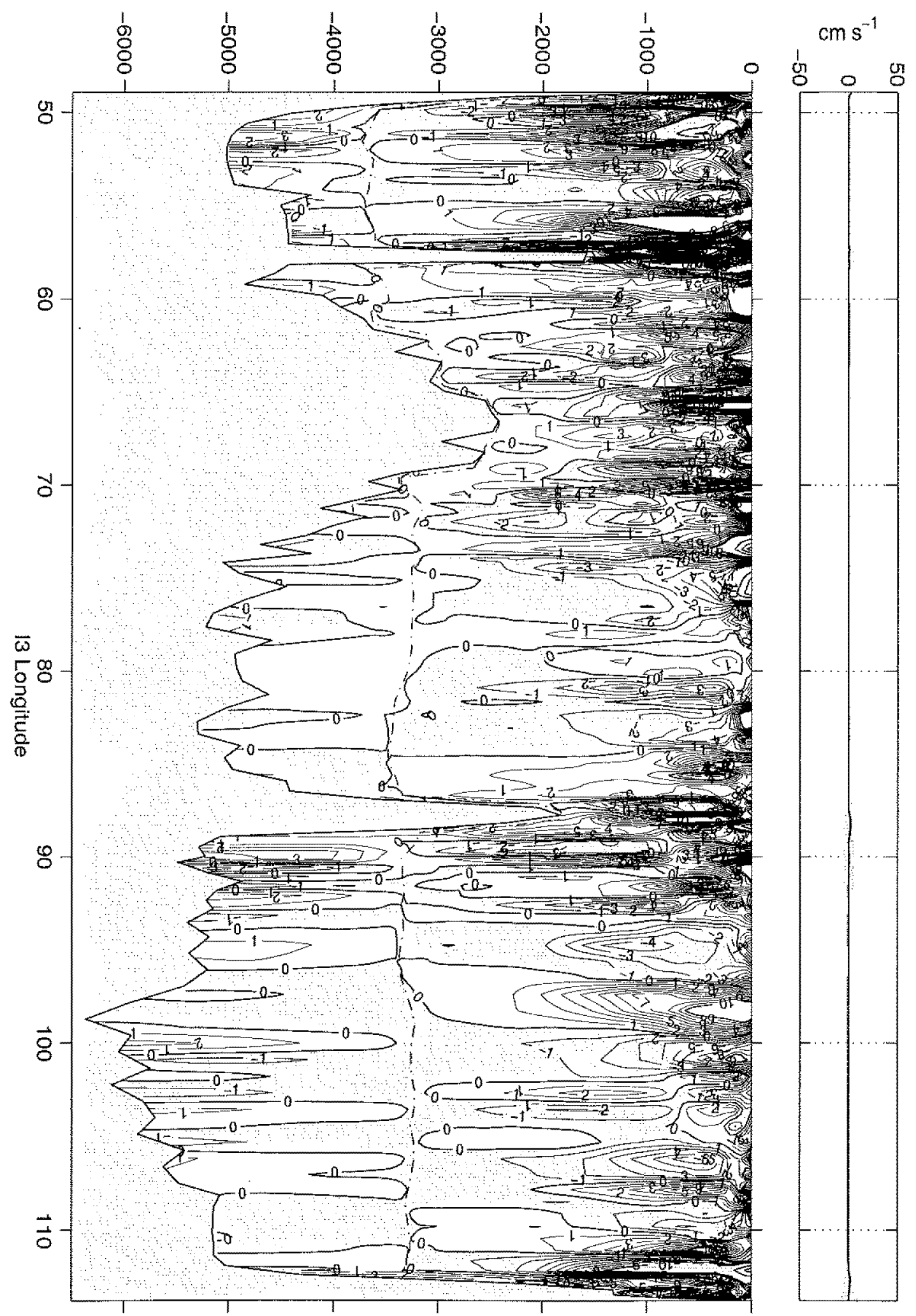

Figure D-5: Absolute velocities, Indian Ocean, I3 (20 $\mathrm{S})$ 
13 , surface to $\Gamma=27.96$
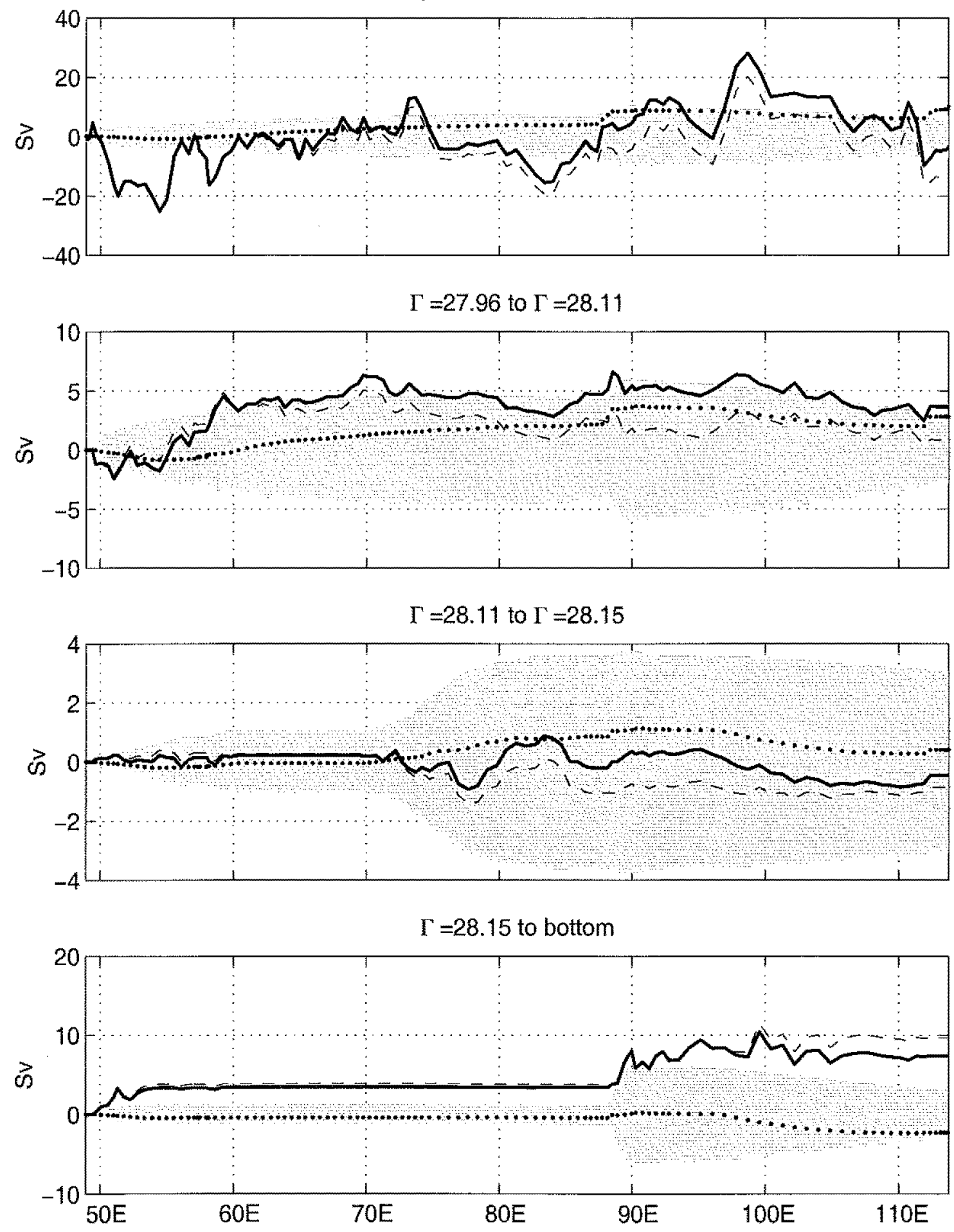

Figure D-6: Cumulative transports, Indian Ocean, I3 (20ㅇ) 


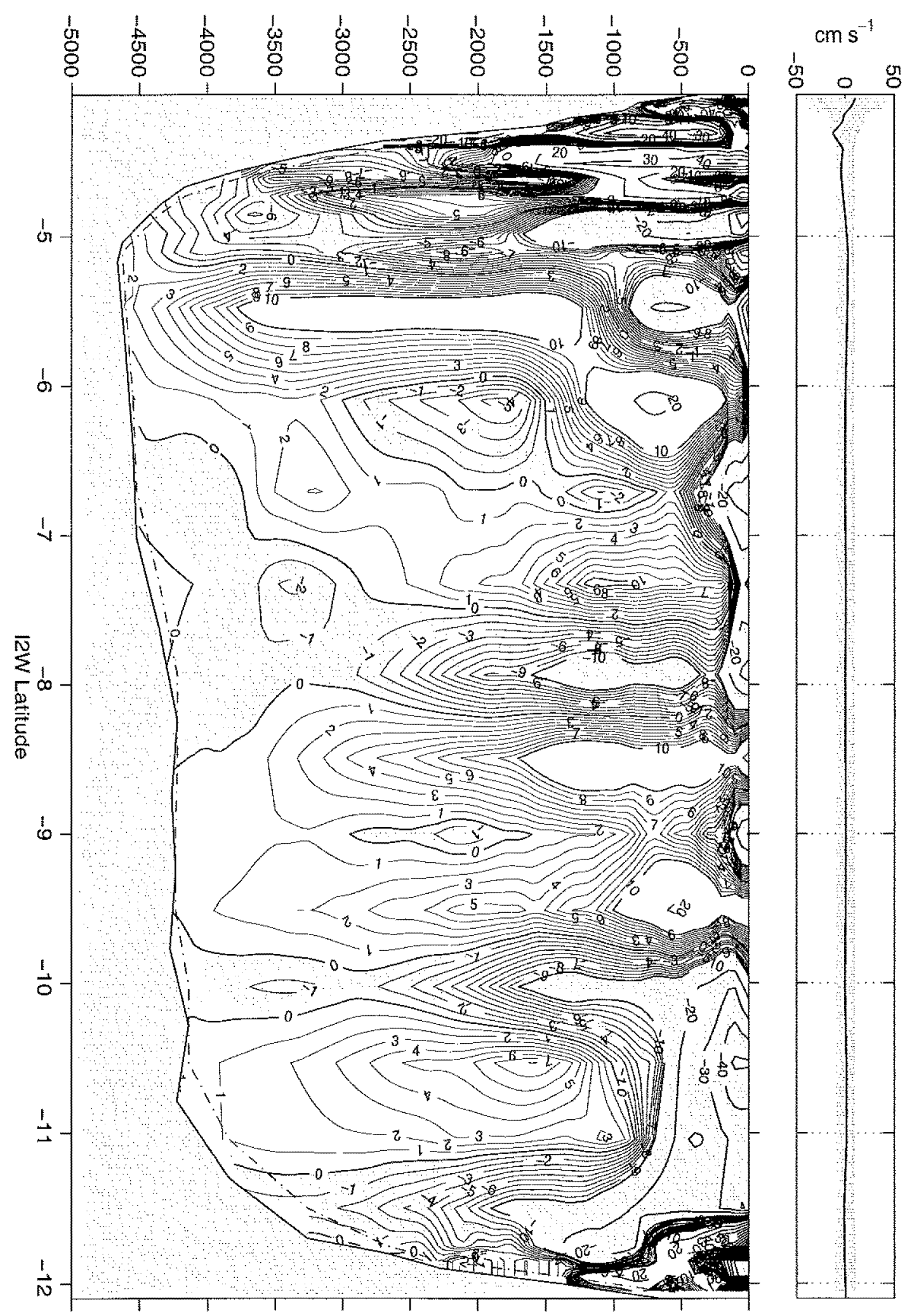

Figure D-7: Absolute velocities, Indian Ocean, $\mathrm{I} 2 \mathrm{~W}\left(45^{\circ} \mathrm{E}\right)$ 

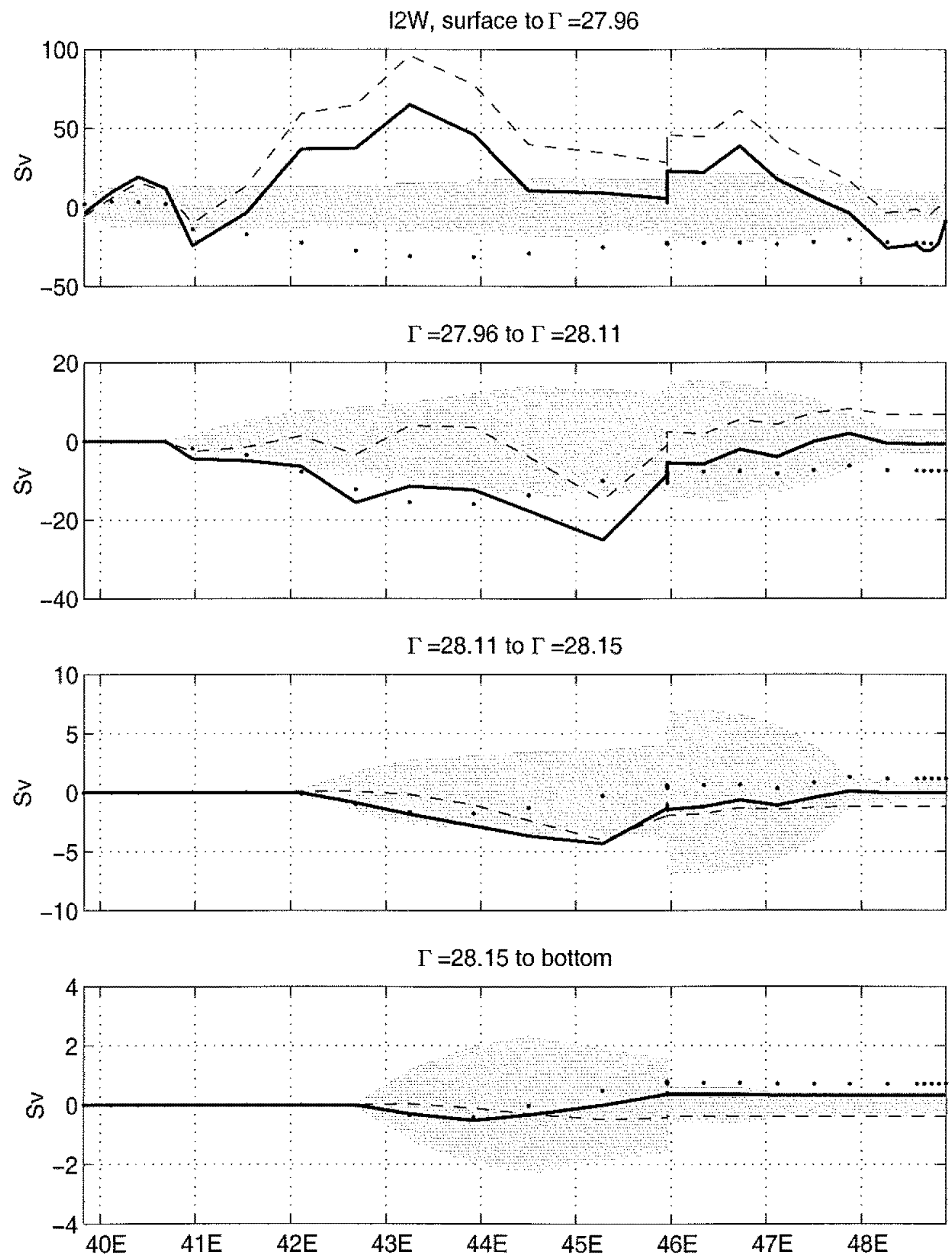

Figure D-8: Cumulative transports, Indian Ocean, I2W (45ㄷ) 


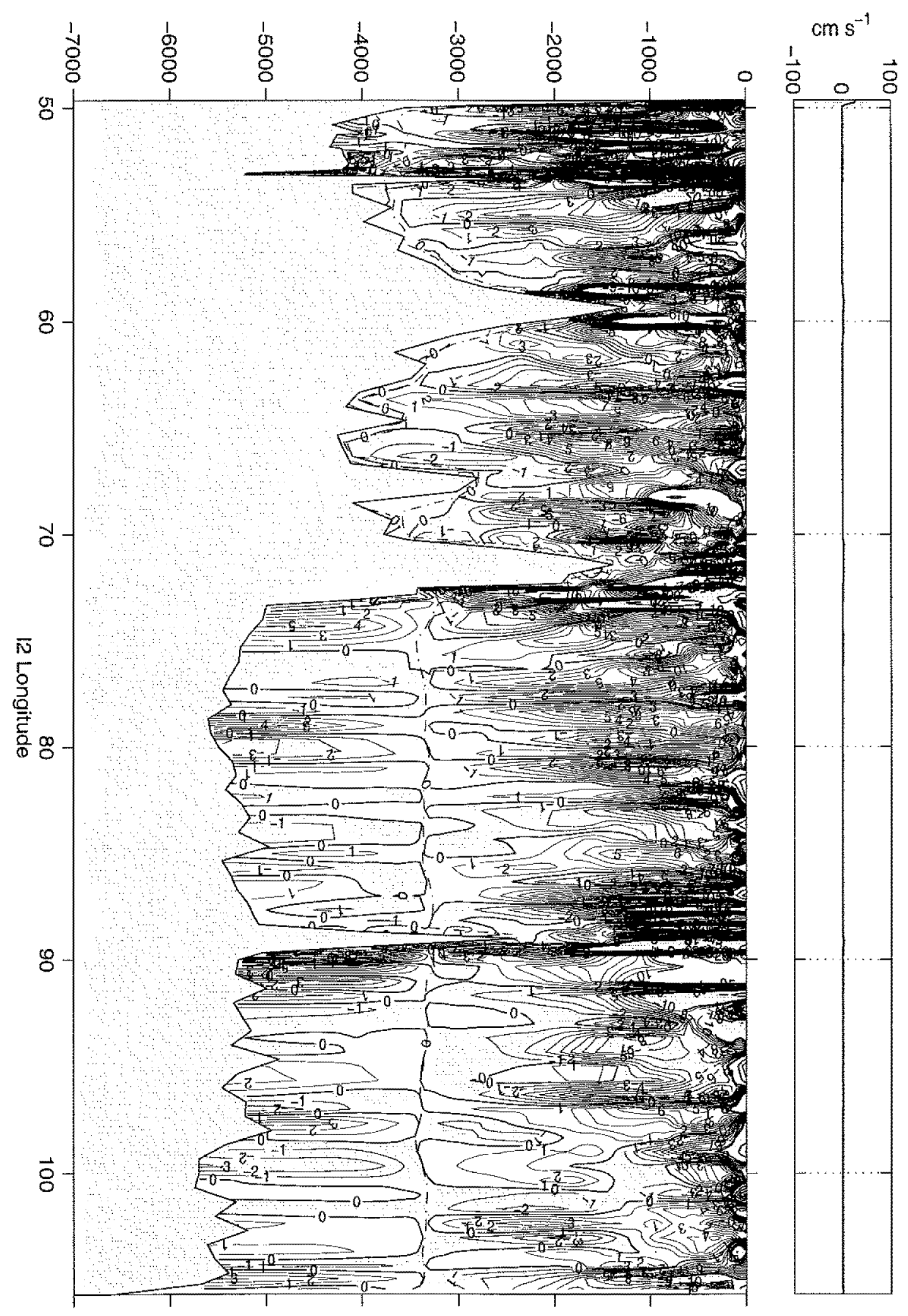

Figure D-9: Absolute velocities, Indian Ocean, I2 ( $\left.8^{\circ} \mathrm{S}\right)$ 

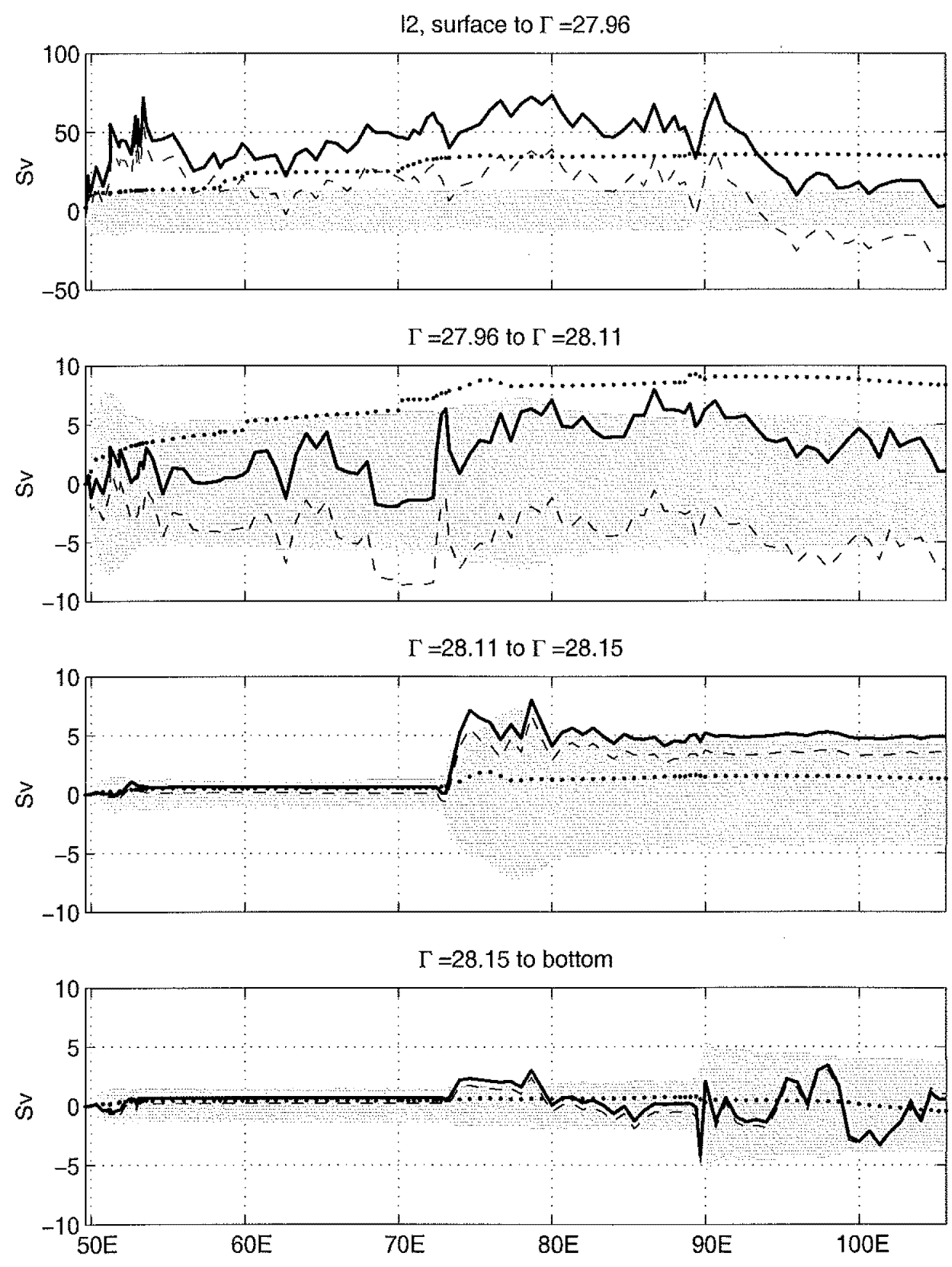

Figure D-10: Cumulative transports, Indian Ocean, I2 ( $\left.8^{\circ} \mathrm{S}\right)$ 


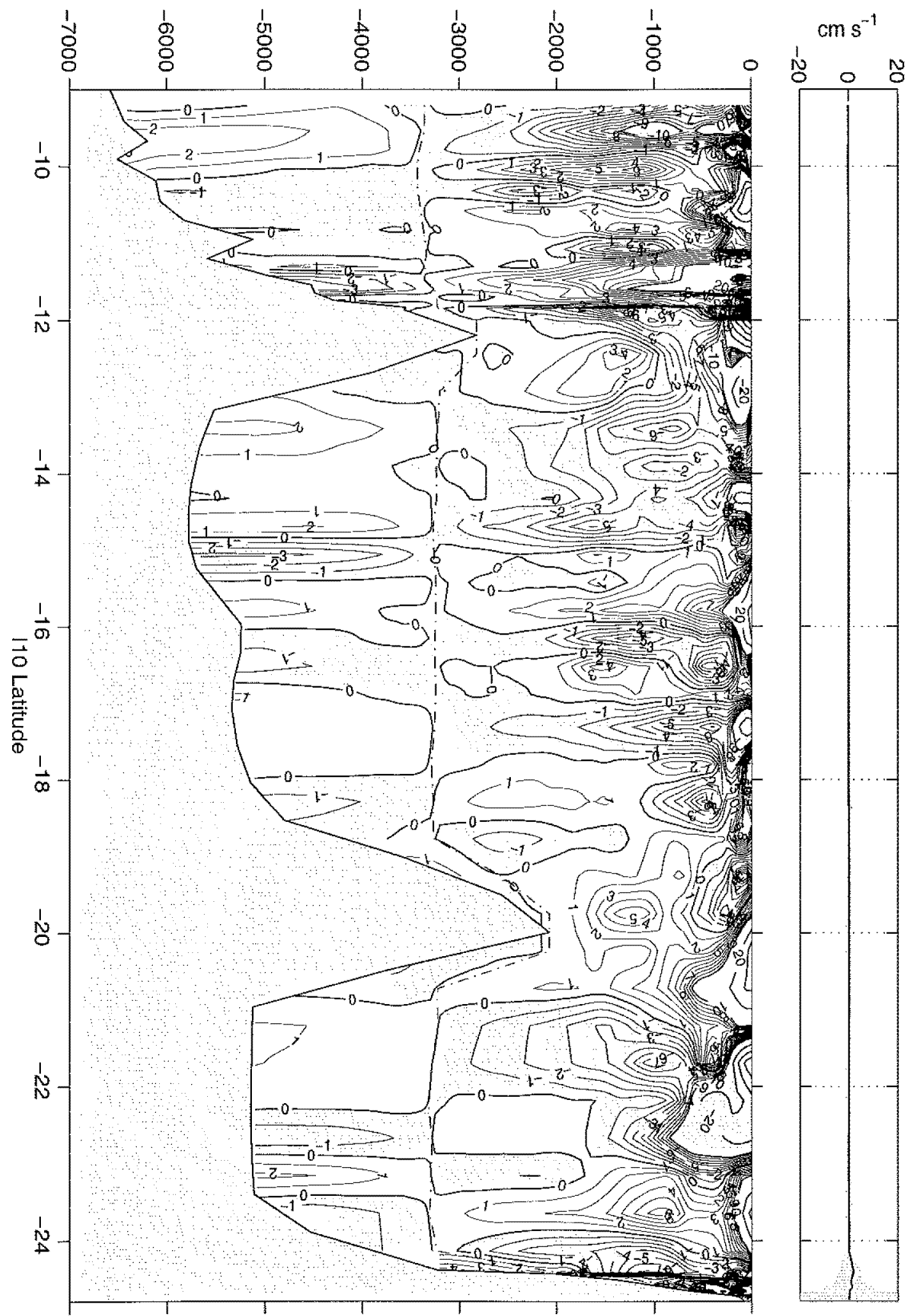

Figure D-11: Absolute velocities, Indian Ocean, I10 (111 $\left.{ }^{\circ} \mathrm{E}\right)$ 

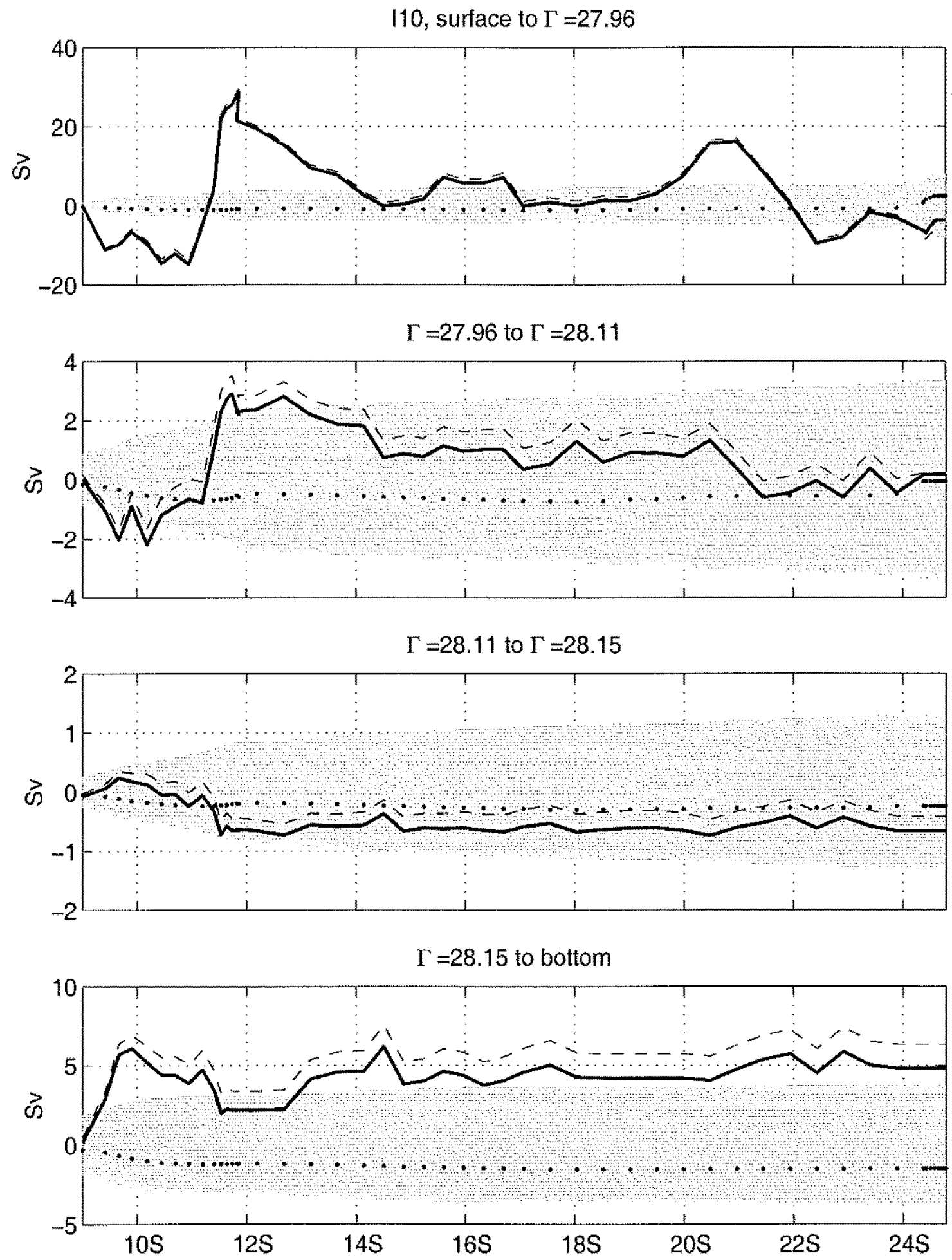

Figure D-12: Cumulative transports, Indian Ocean, $110\left(111^{\circ} \mathrm{E}\right)$ 


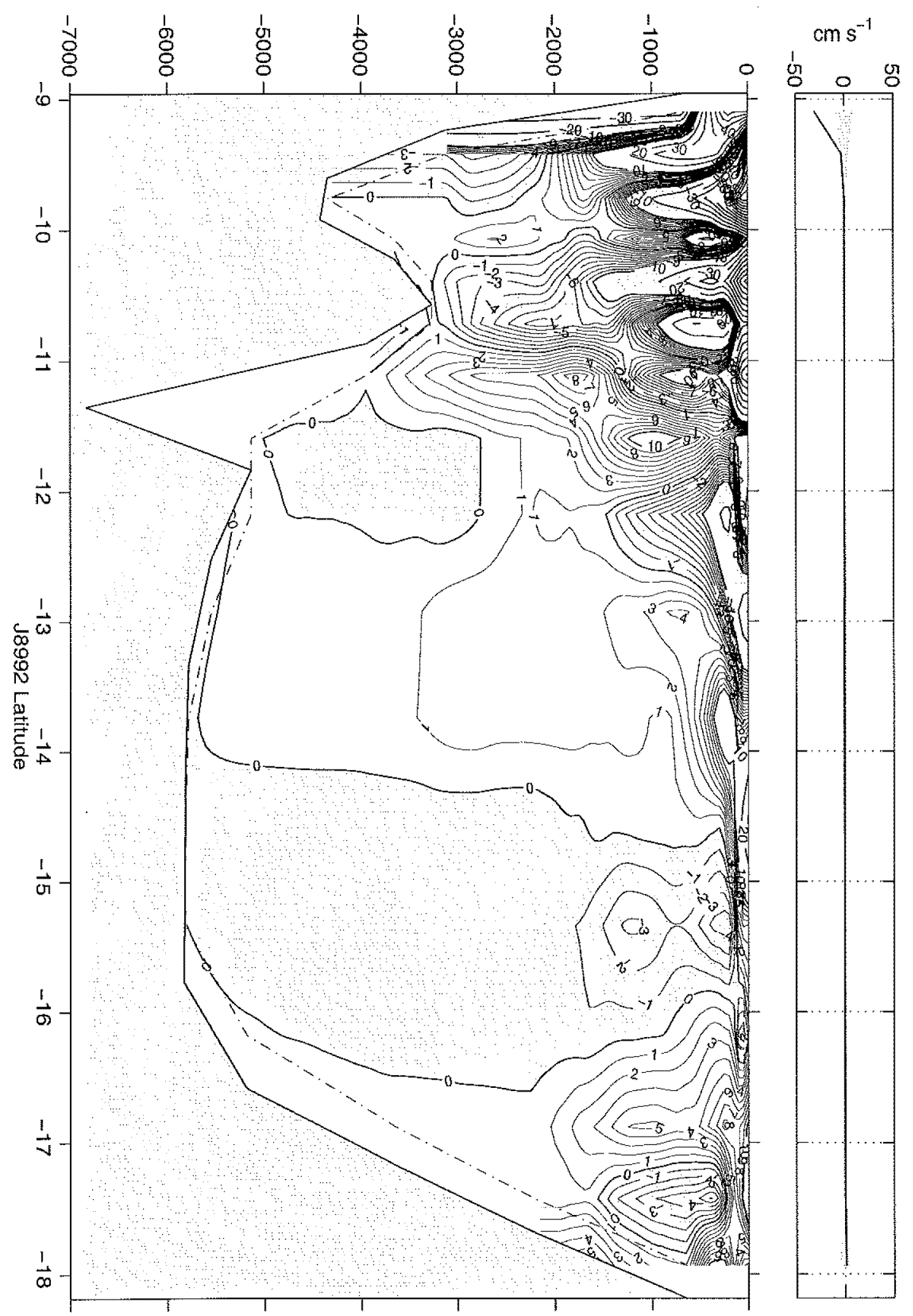

Figure D-13: Absolute velocities, Indian Ocean, $J 8992\left(116^{\circ} \mathrm{E}\right)$ 

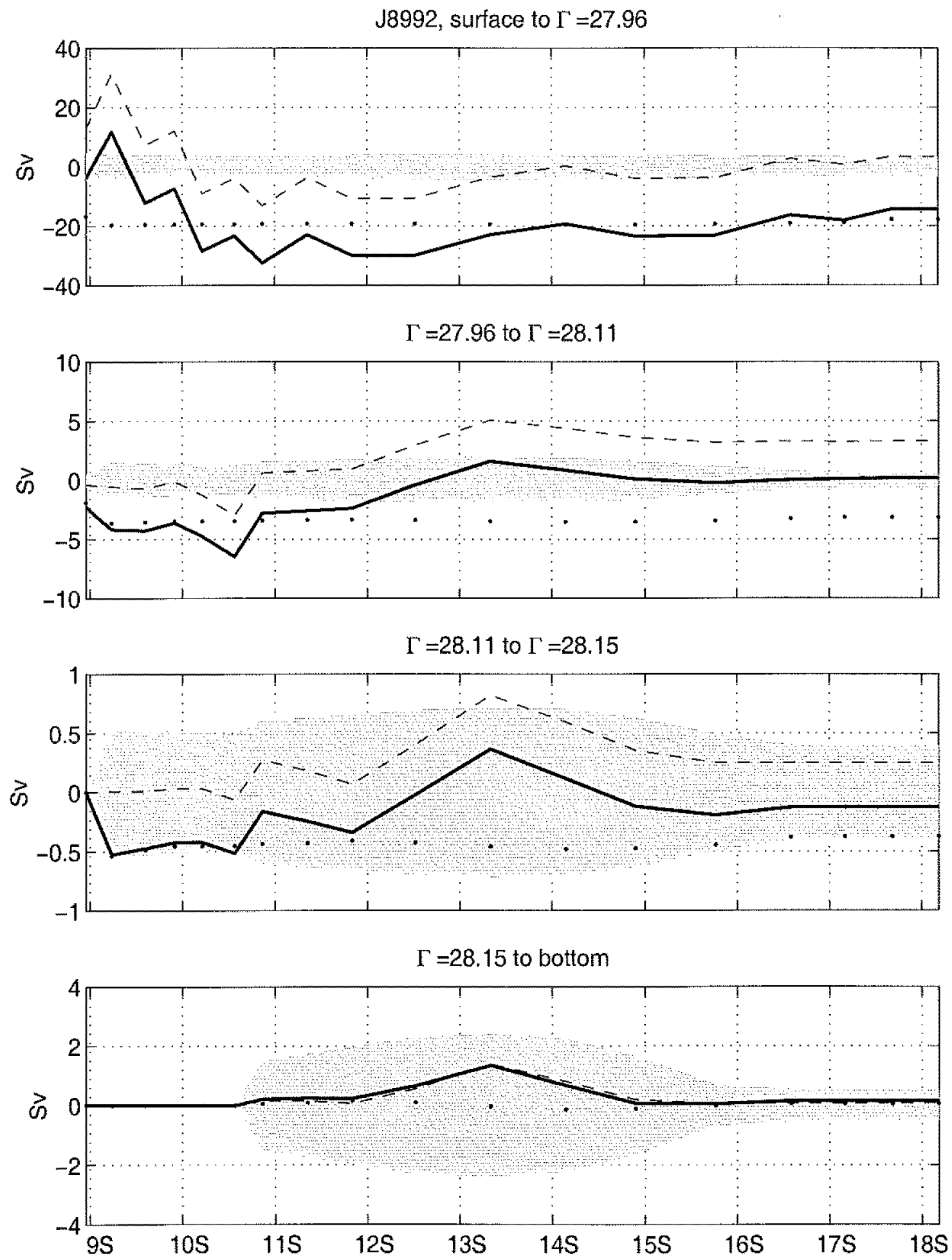

Figure D-14: Cumulative transports, Indian Ocean, J8992 (116 $\left.{ }^{\circ} \mathrm{E}\right)$ 


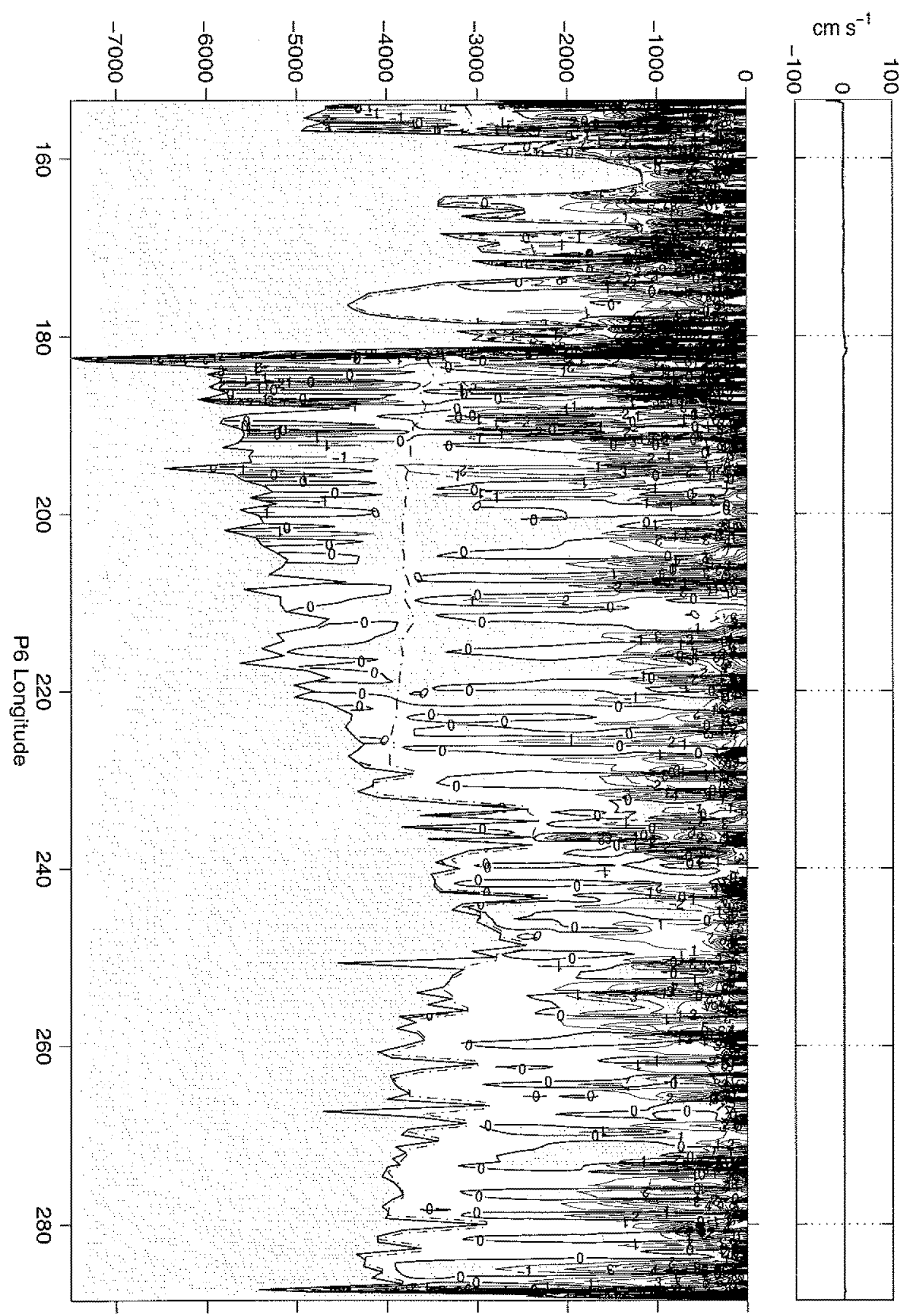

Figure D-15: Absolute velocities, Pacific Ocean, P6 (30 S) 

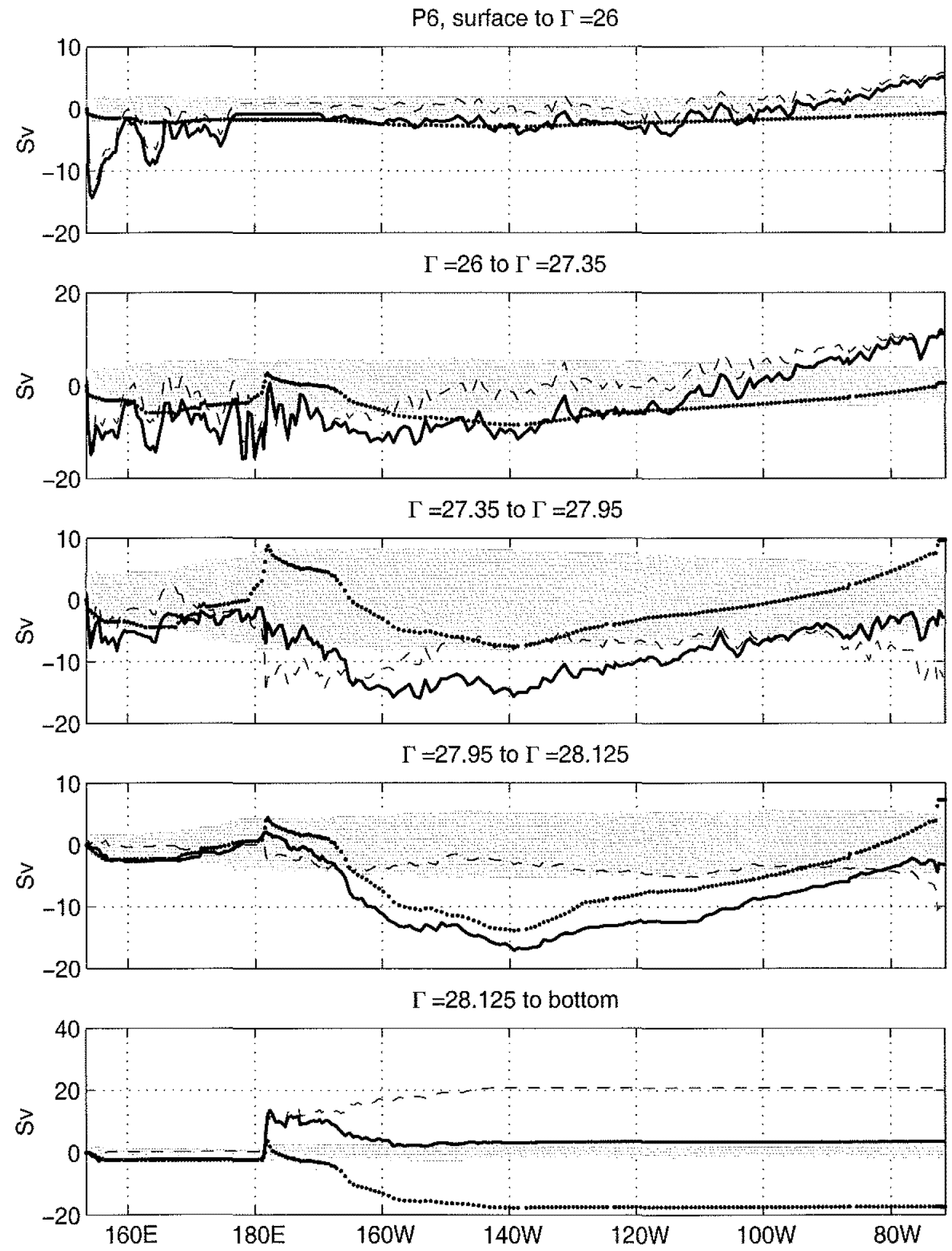

Figure D-16: Cumulative transports, Pacific Ocean, P6 $\left(30^{\circ} \mathrm{S}\right)$ 


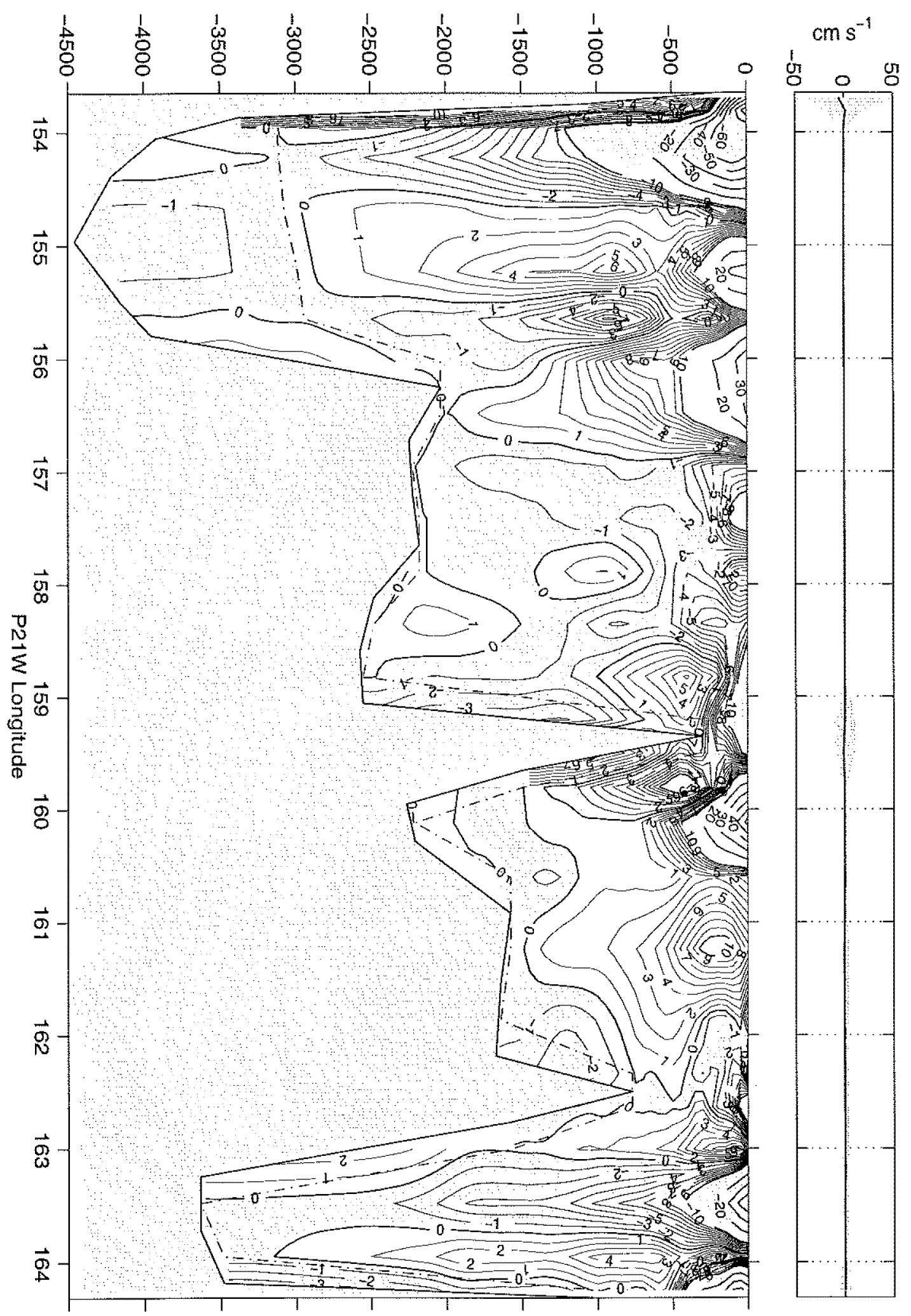

Figure D-17: Absolute velocities, Pacific Ocean, P21W (17\% S, Australia to New Caledonia). 

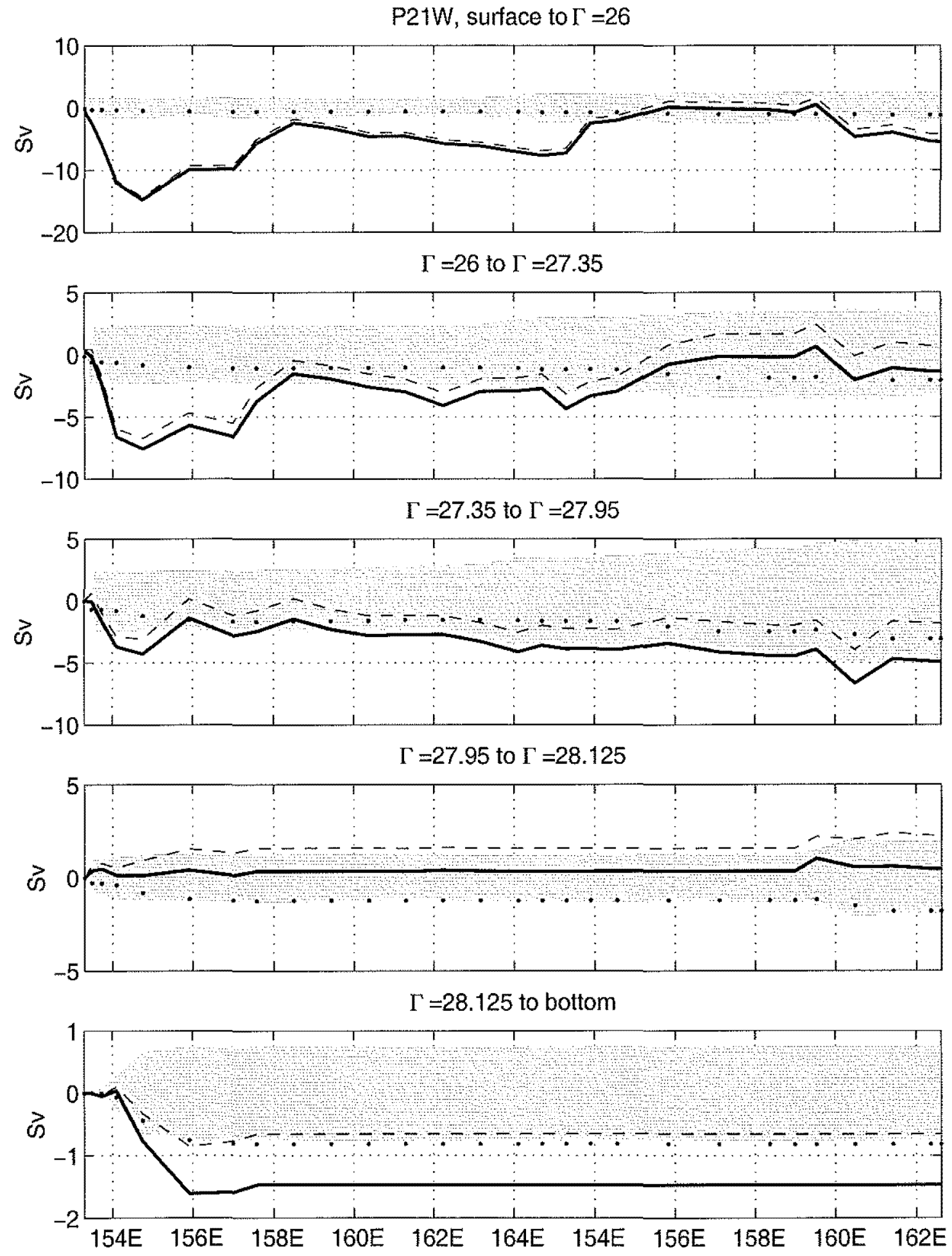

Figure D-18: Cumulative transports, Pacific Ocean, P21W $\left(17^{\circ} \mathrm{S}\right.$, Australia to New Caledonia). 


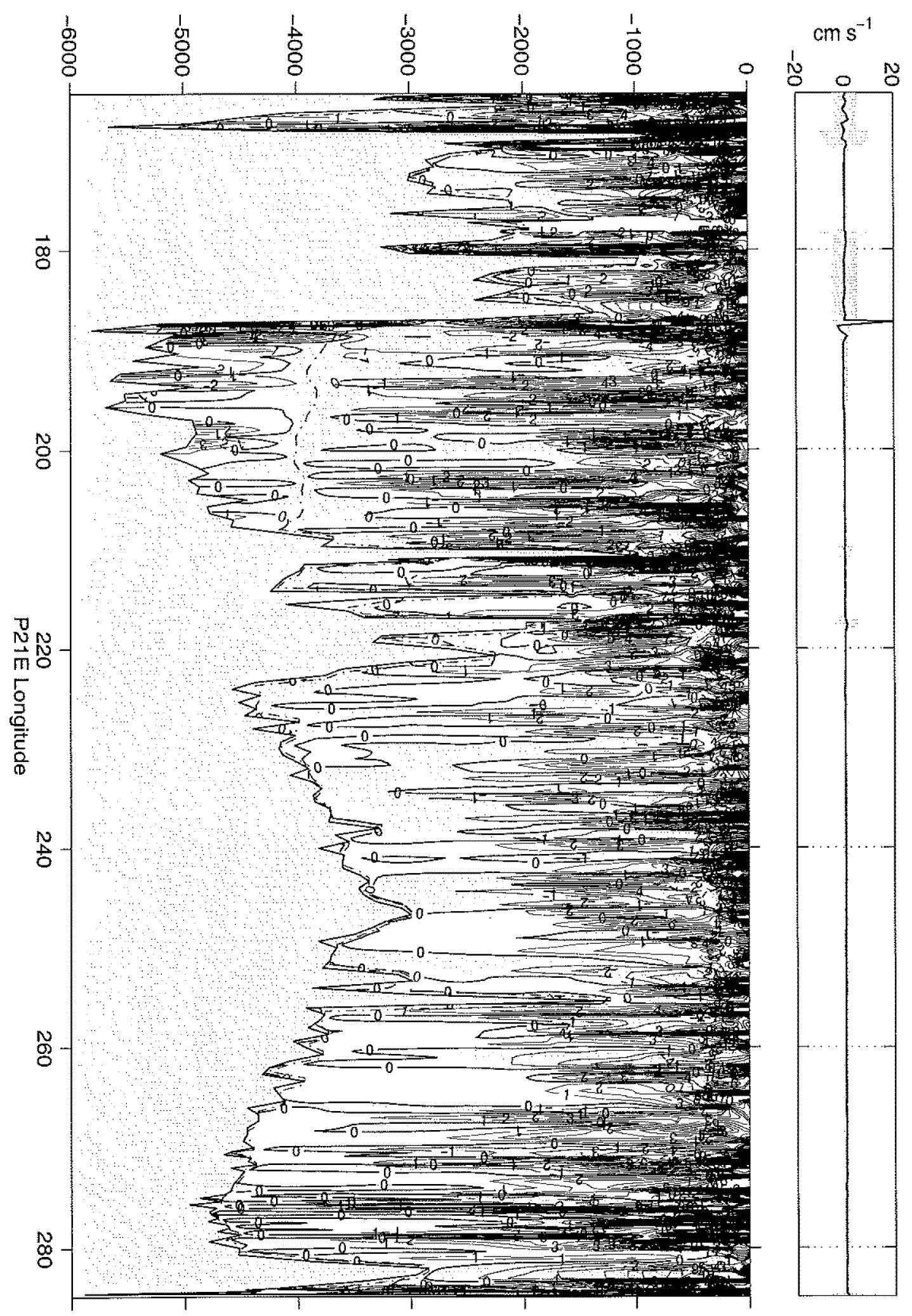

Figure D-19: Absolute velocities, Pacific Ocean, P21E $\left(17^{\circ} \mathrm{S}\right.$, New Caledonia to South America). 

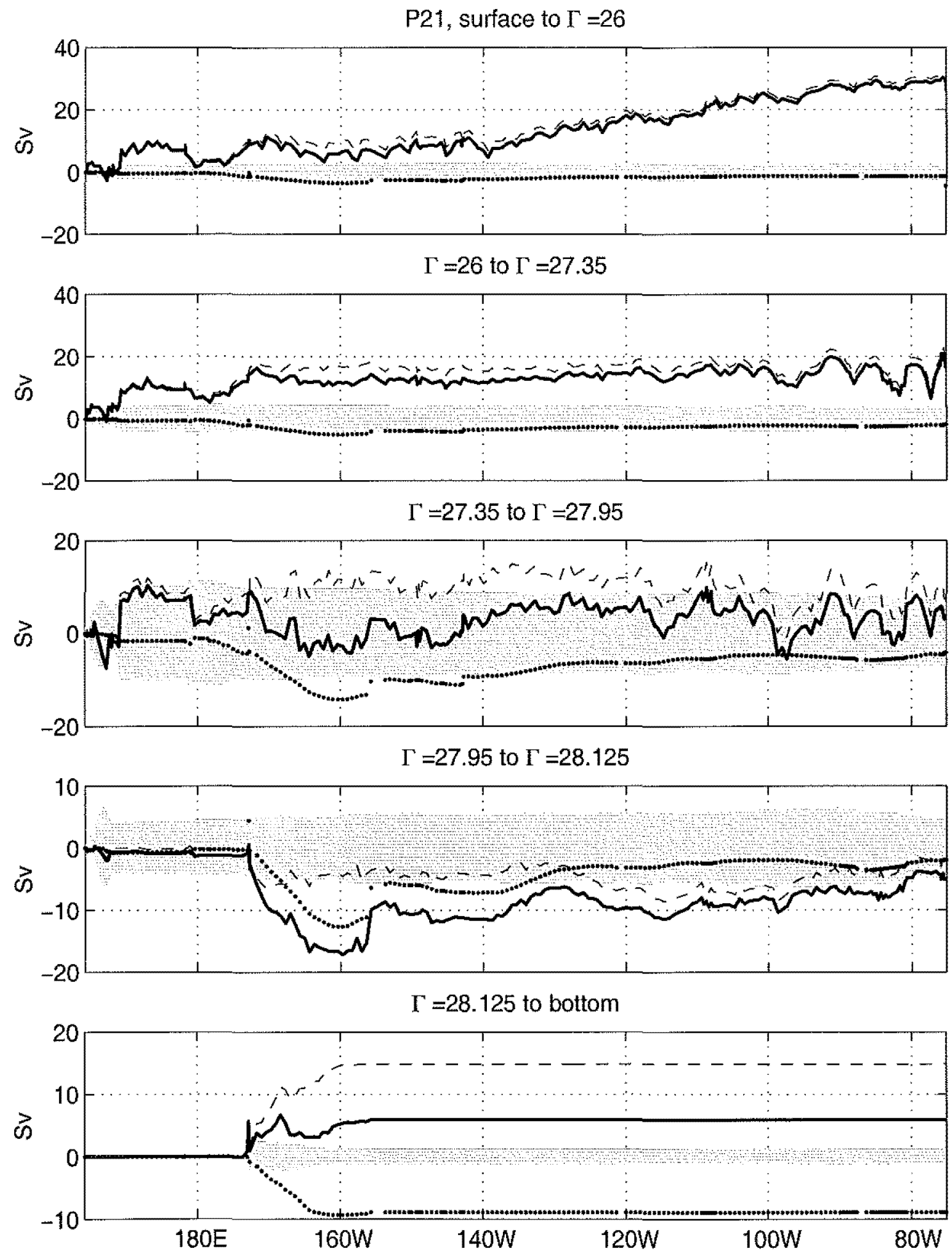

Figure D-20: Cumulative transports, Pacific Ocean, P21E $\left(17^{\circ} \mathrm{S}\right.$, New Caledonia to South America). 


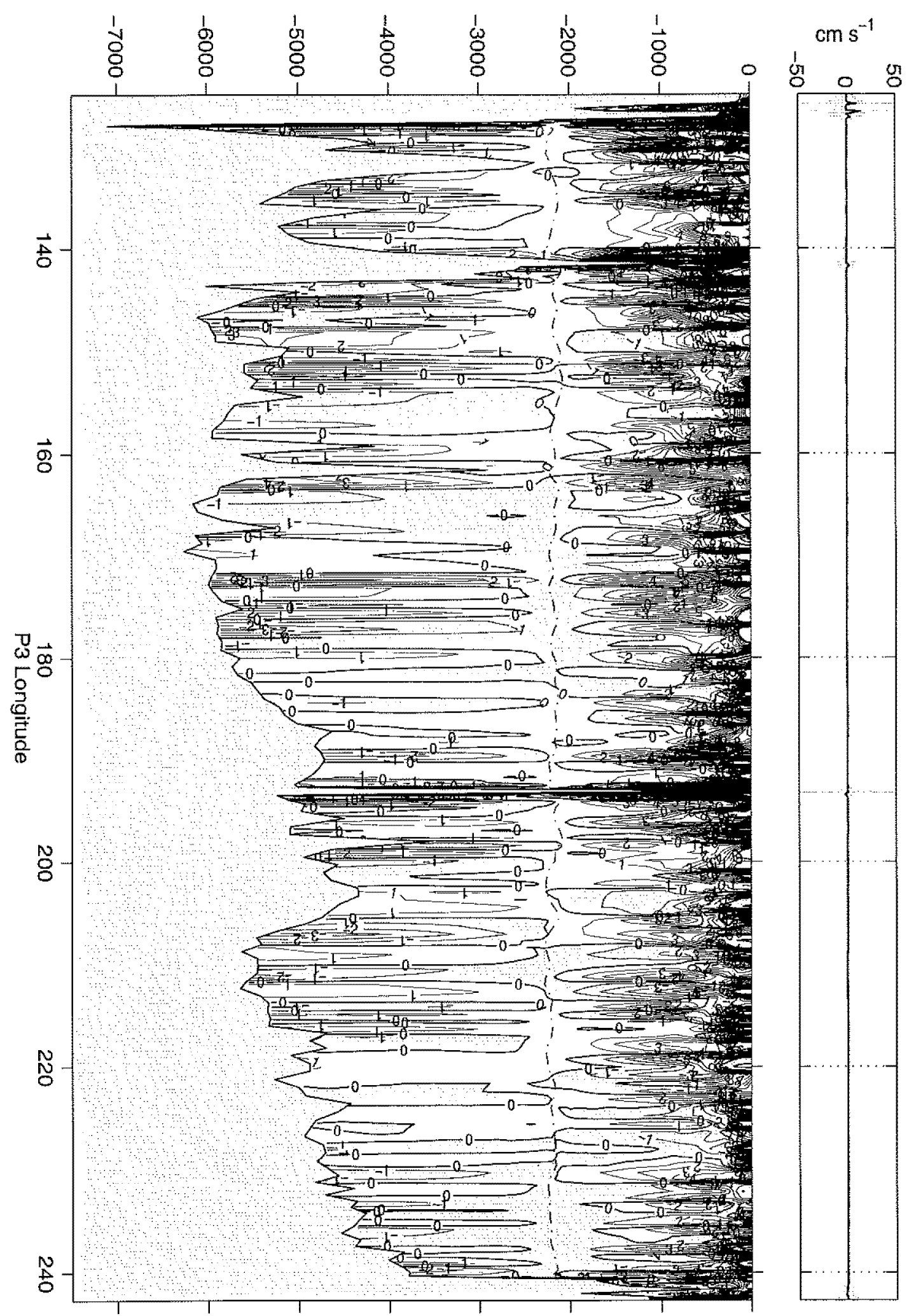

Figure D-21: Absolute velocities, Pacific Ocean, P3 (24N). 

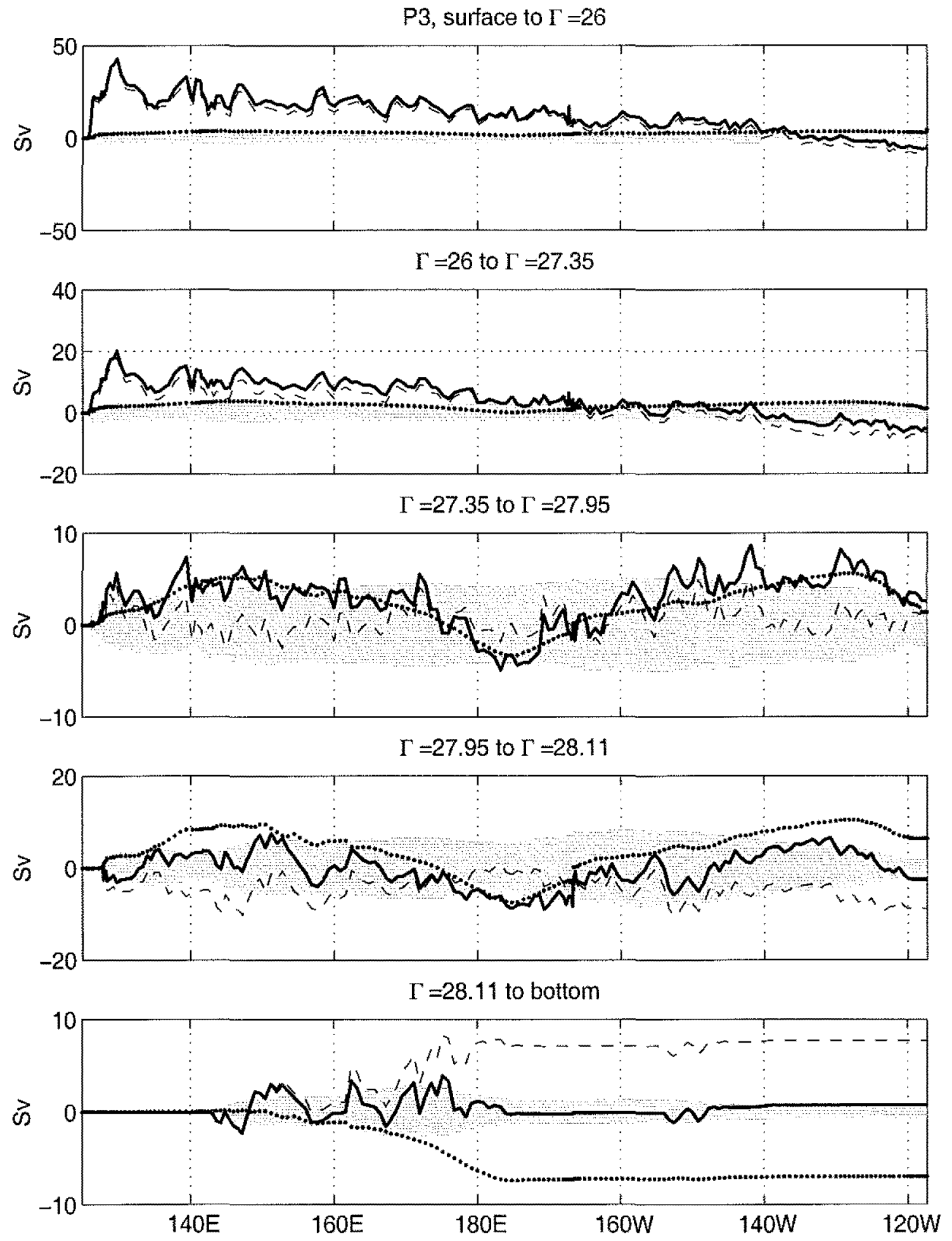

Figure D-22: Cumulative transports, Pacific Ocean, P3 $\left(24^{\circ} \mathrm{N}\right)$. 


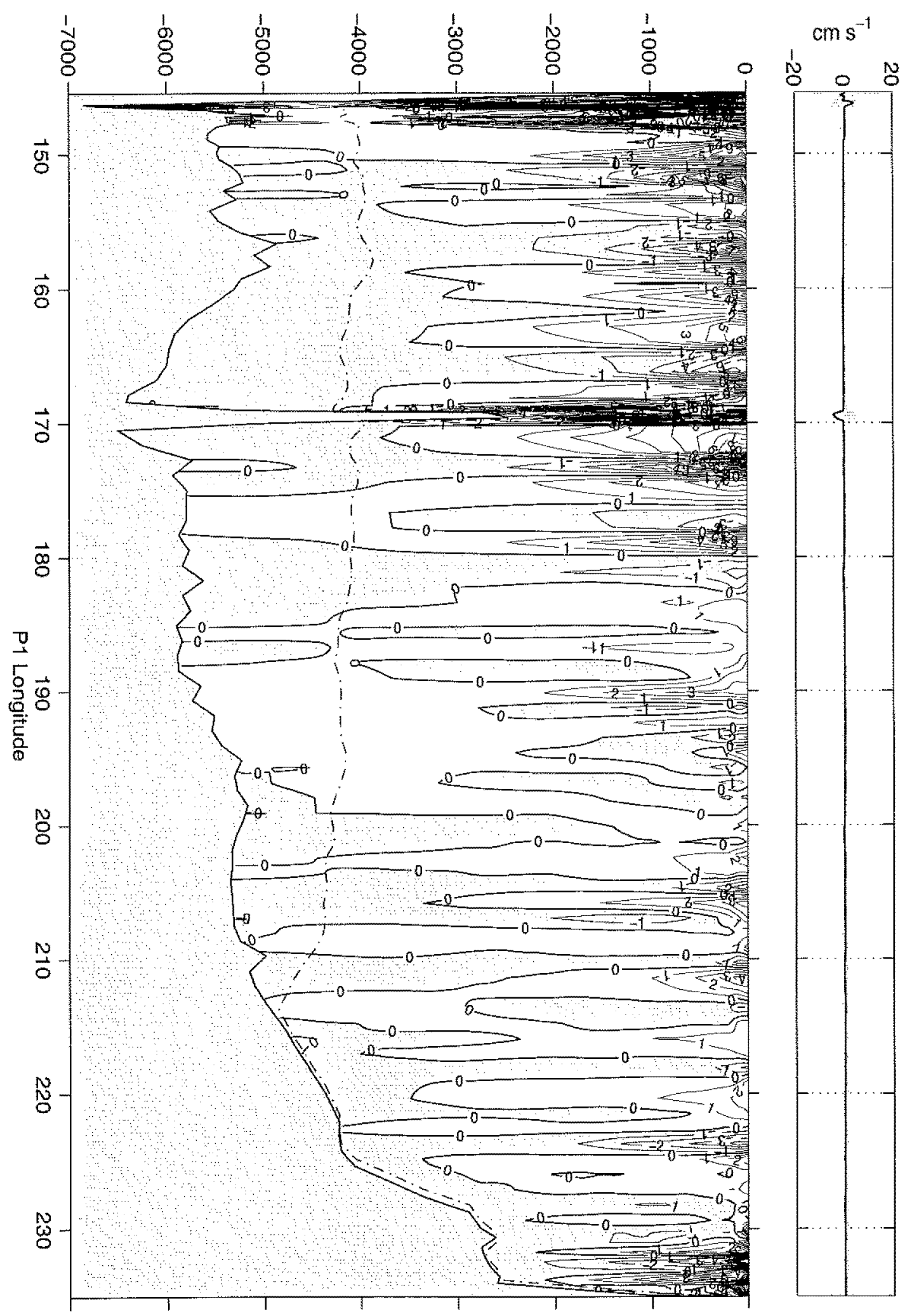

Figure D-23: Absolute velocities, Pacific Ocean, P1 (47 N). 

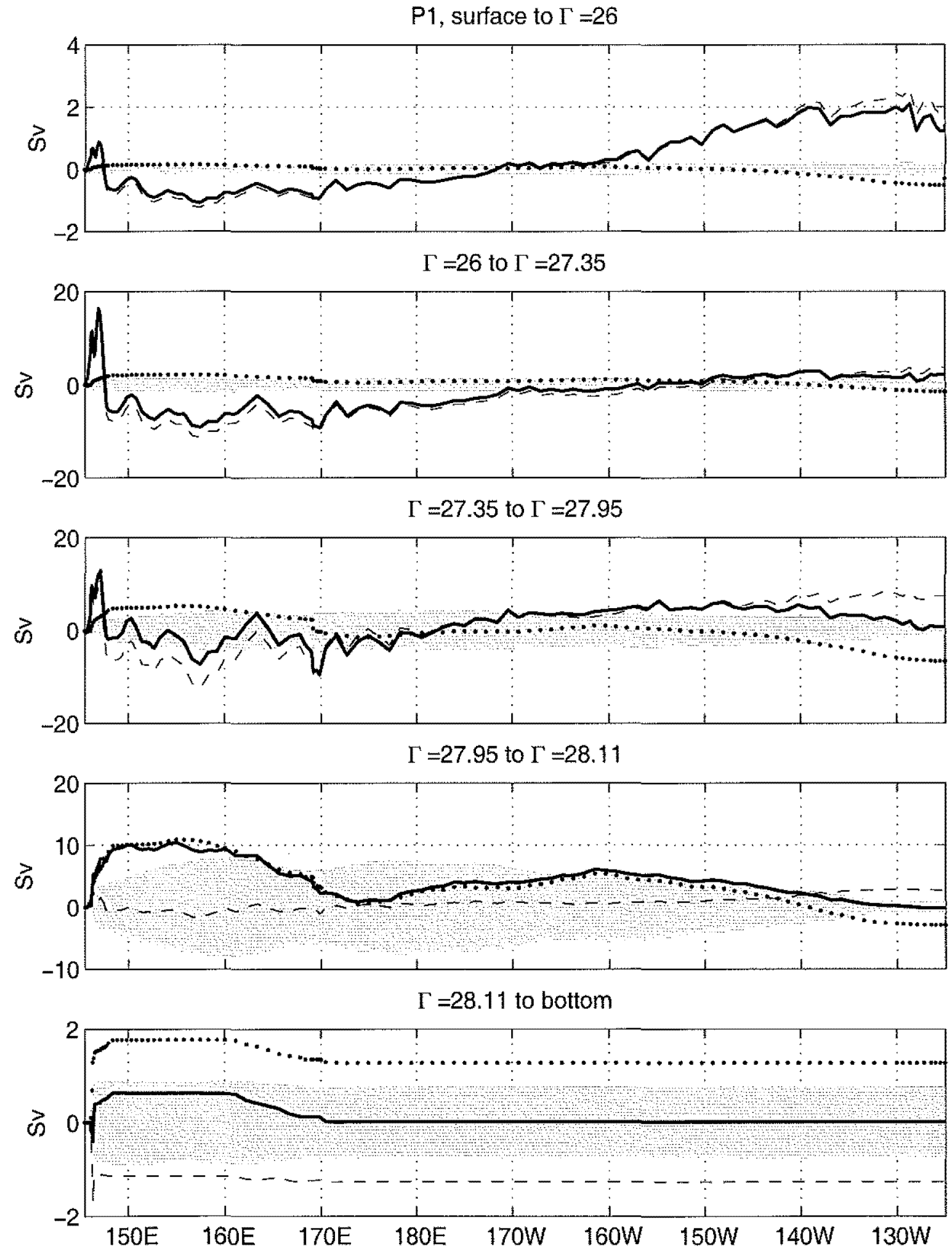

Figure D-24: Cumulative transports, Pacific Ocean, P1 $\left(47^{\circ} \mathrm{N}\right)$. 


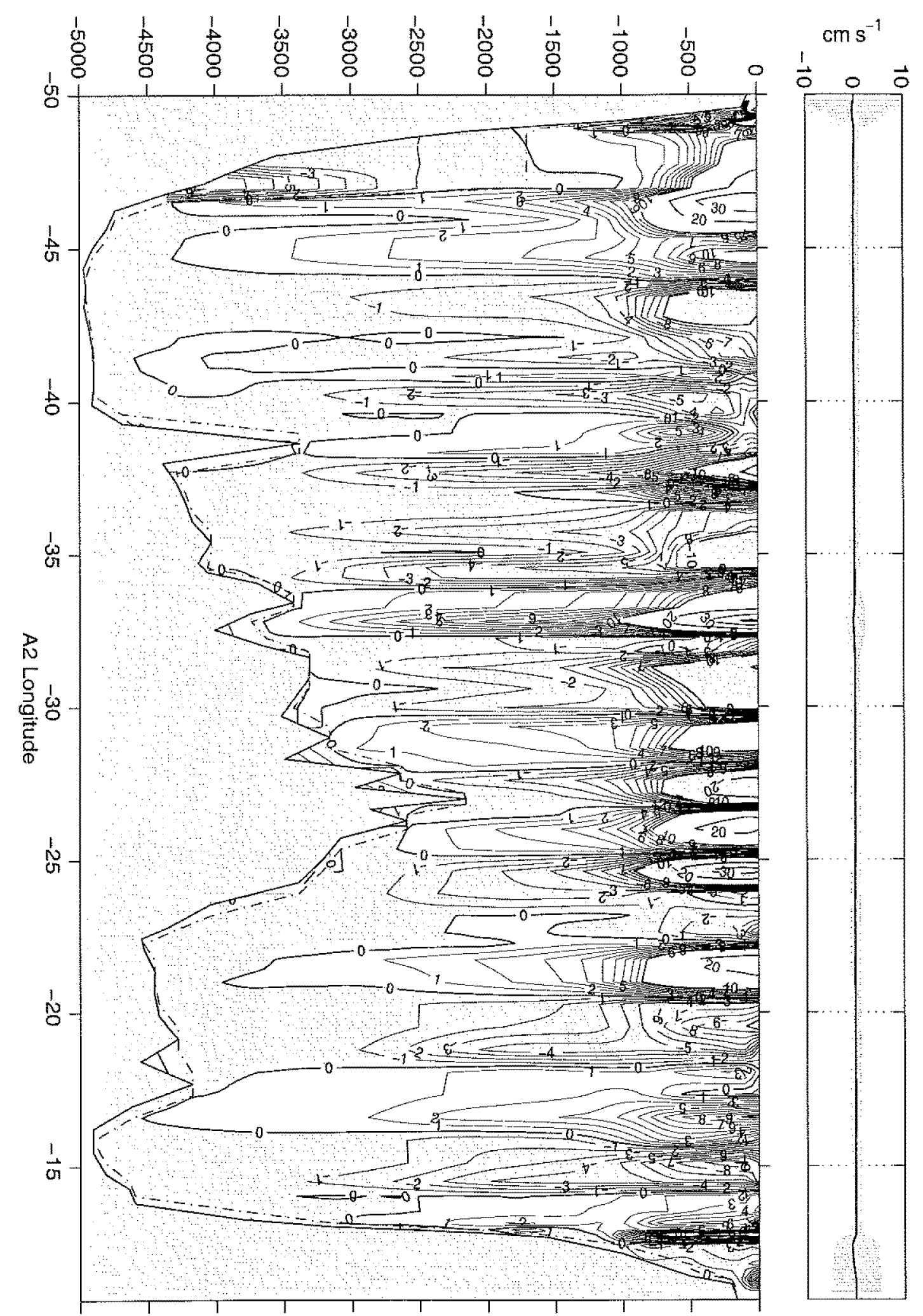

Figure D-25: Absolute velocities, Atlantic Ocean, A2 ( $\left.48^{\circ} \mathrm{N}\right)$. 

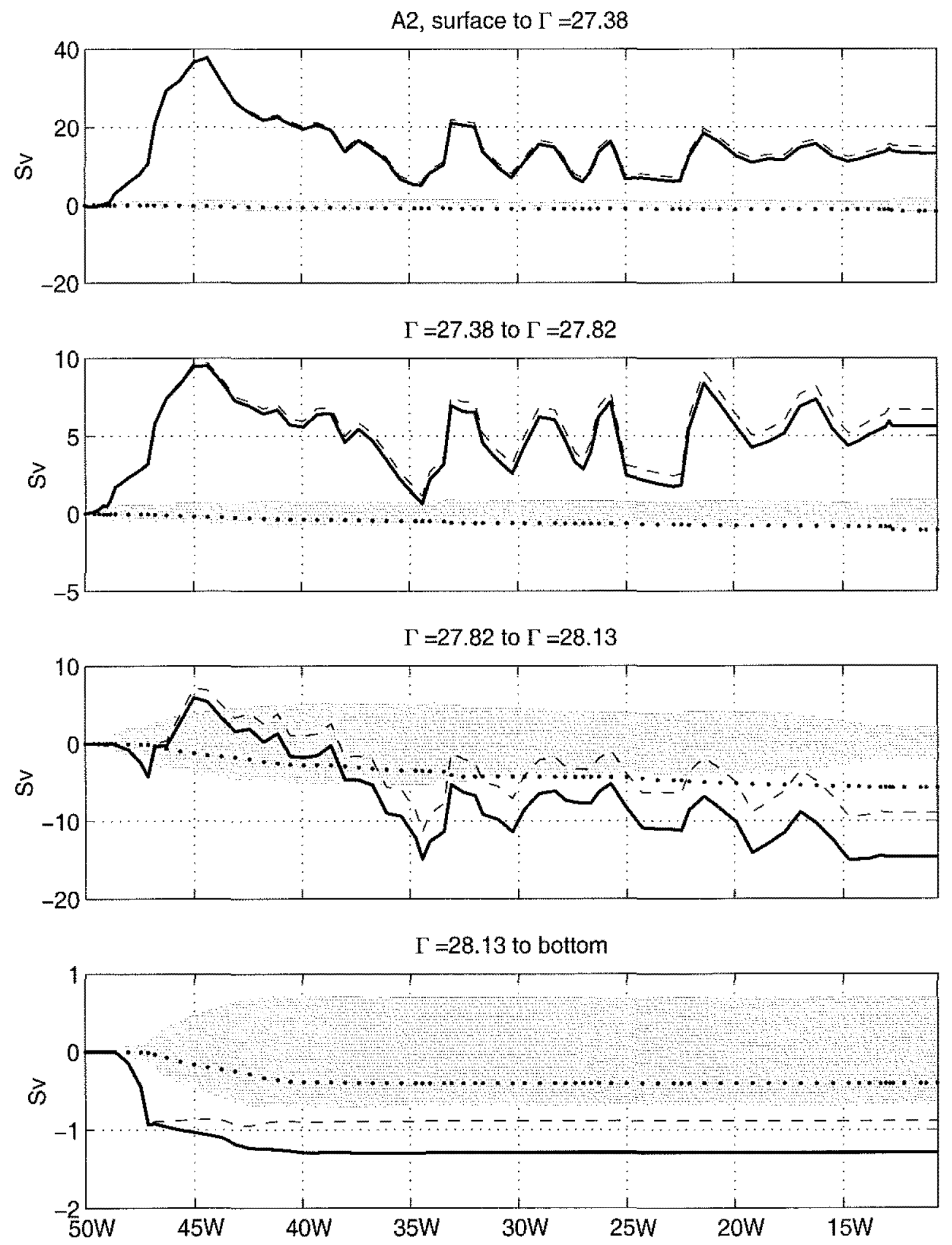

Figure D-26: Cumulative transports, Atlantic Ocean, A2 (48 $\left.{ }^{\circ} \mathrm{N}\right)$. 


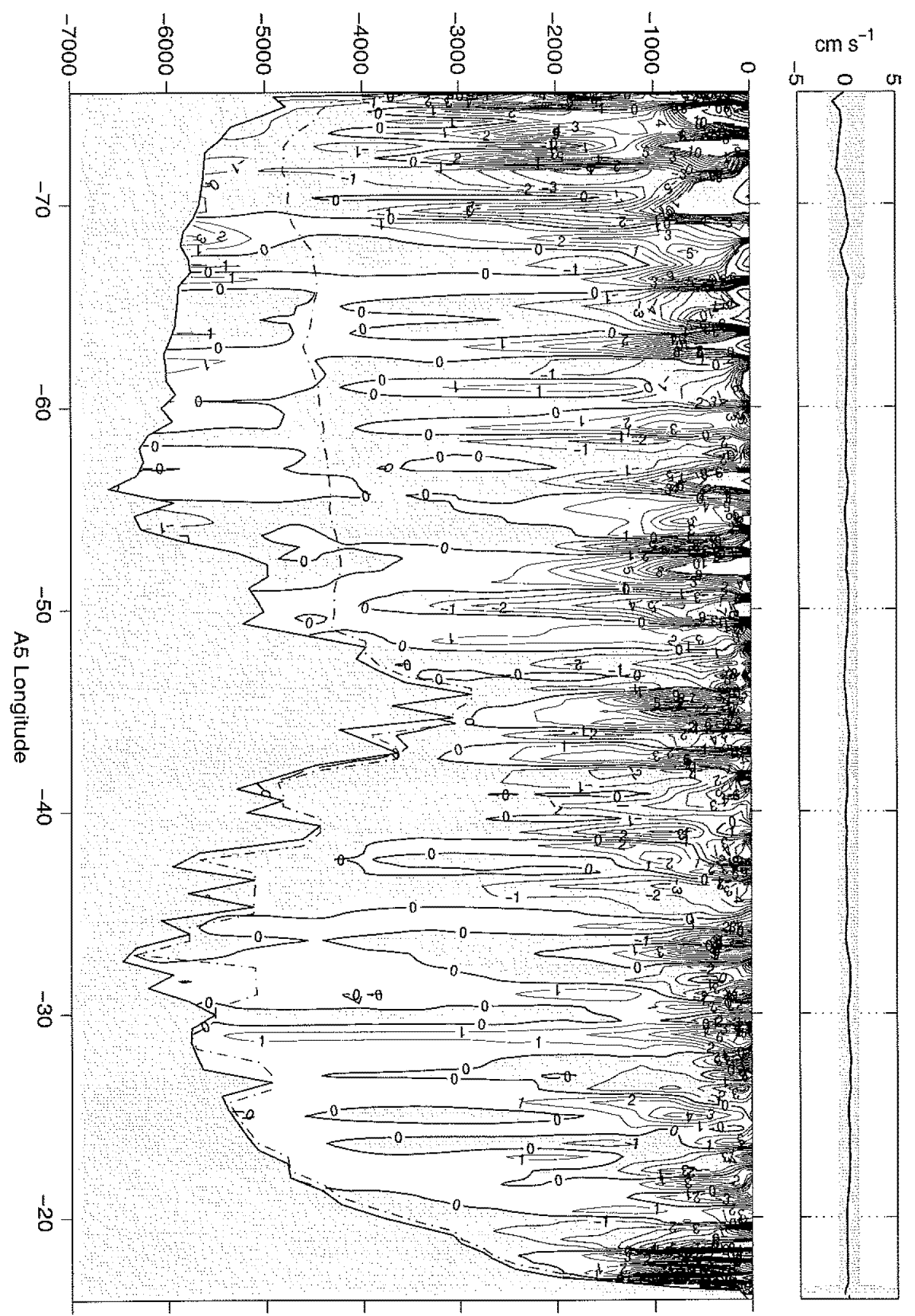

Figure D-27: Absolute velocities, Atlantic Ocean, A5 (24요. 

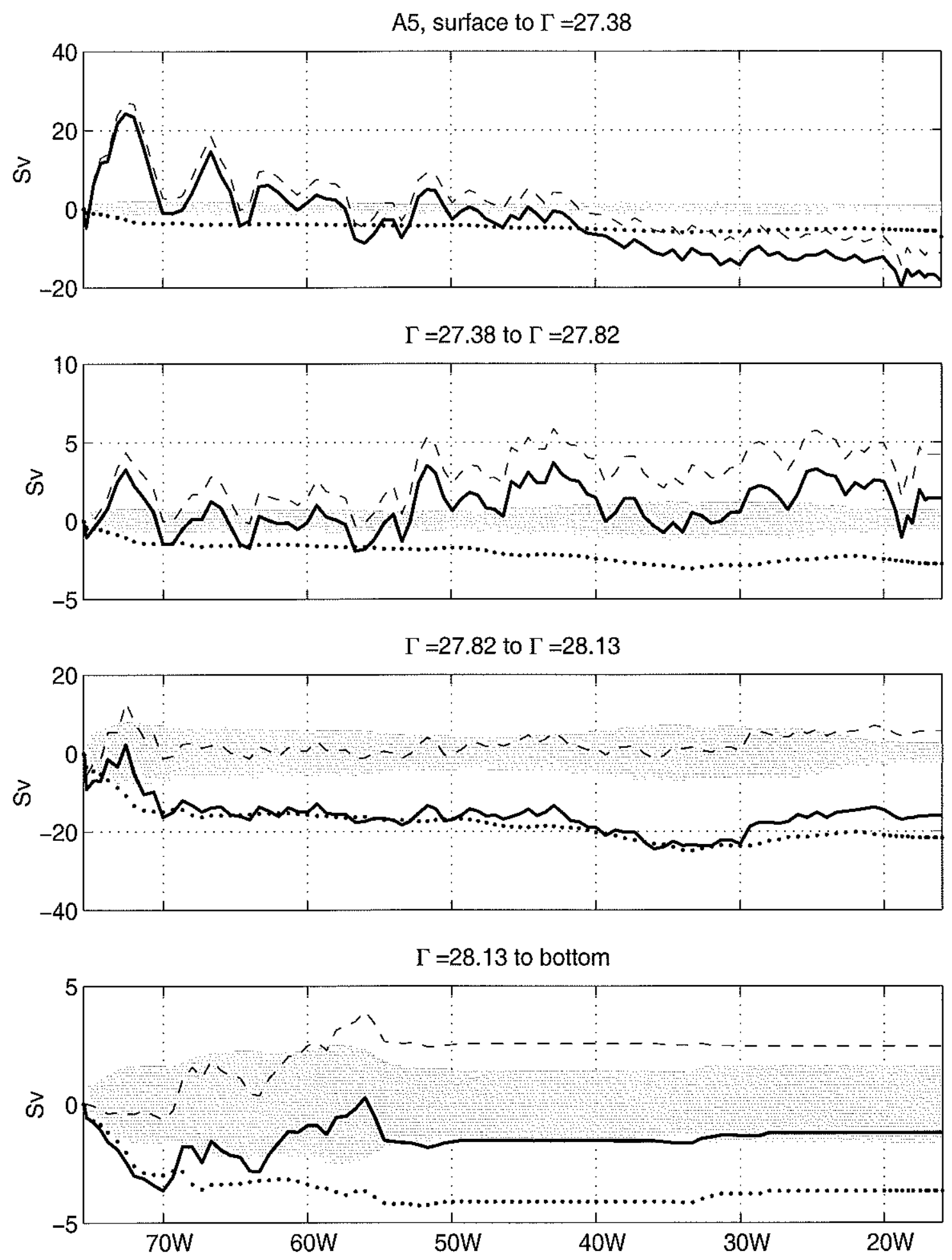

Figure D-28: Cumulative transports, Atlantic Ocean, A5 $\left(24^{\circ} \mathrm{N}\right)$. 


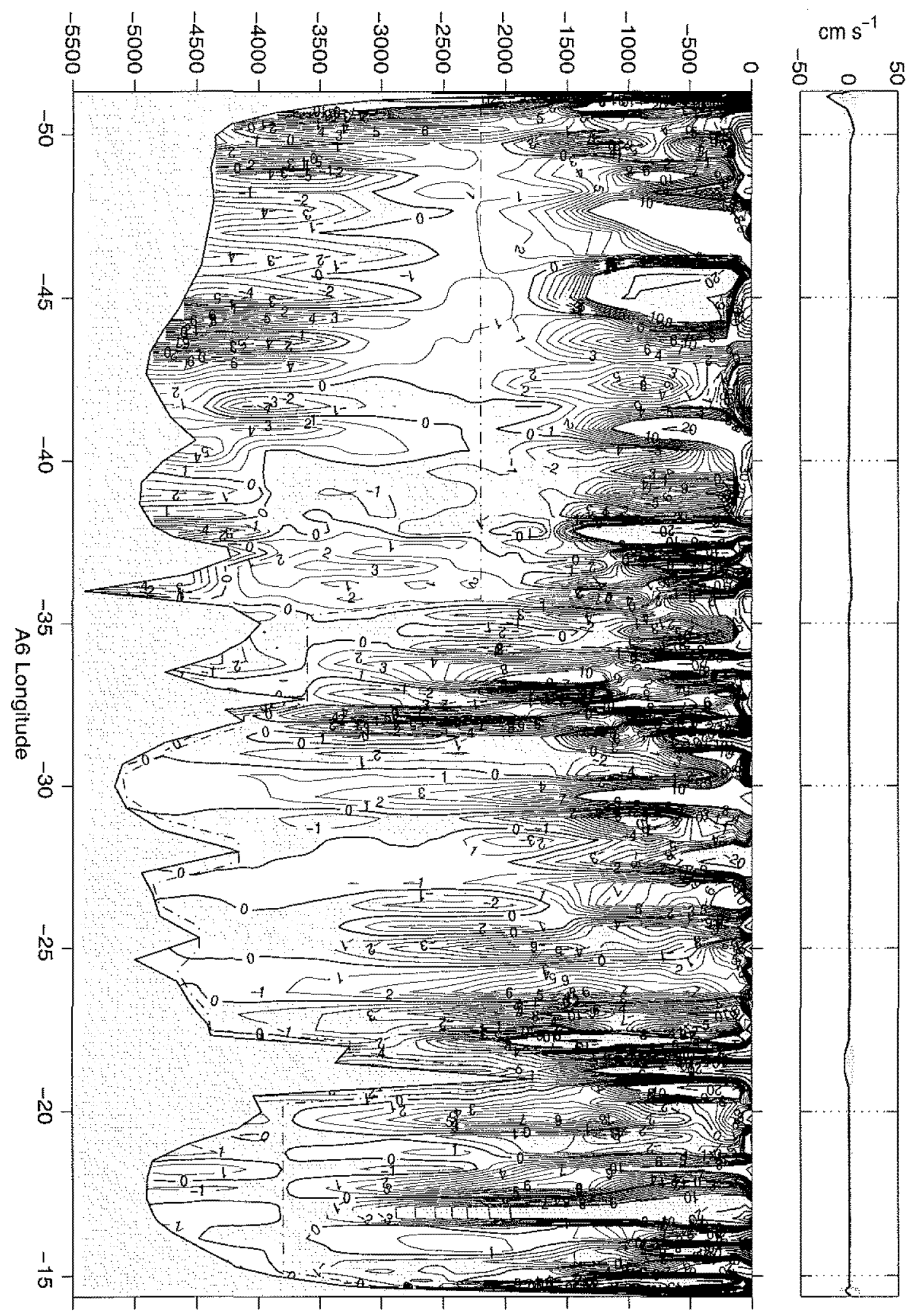

Figure D-29: Absolute velocities, Atlantic Ocean, A6 $\left(7.5^{\circ} \mathrm{N}\right)$. 

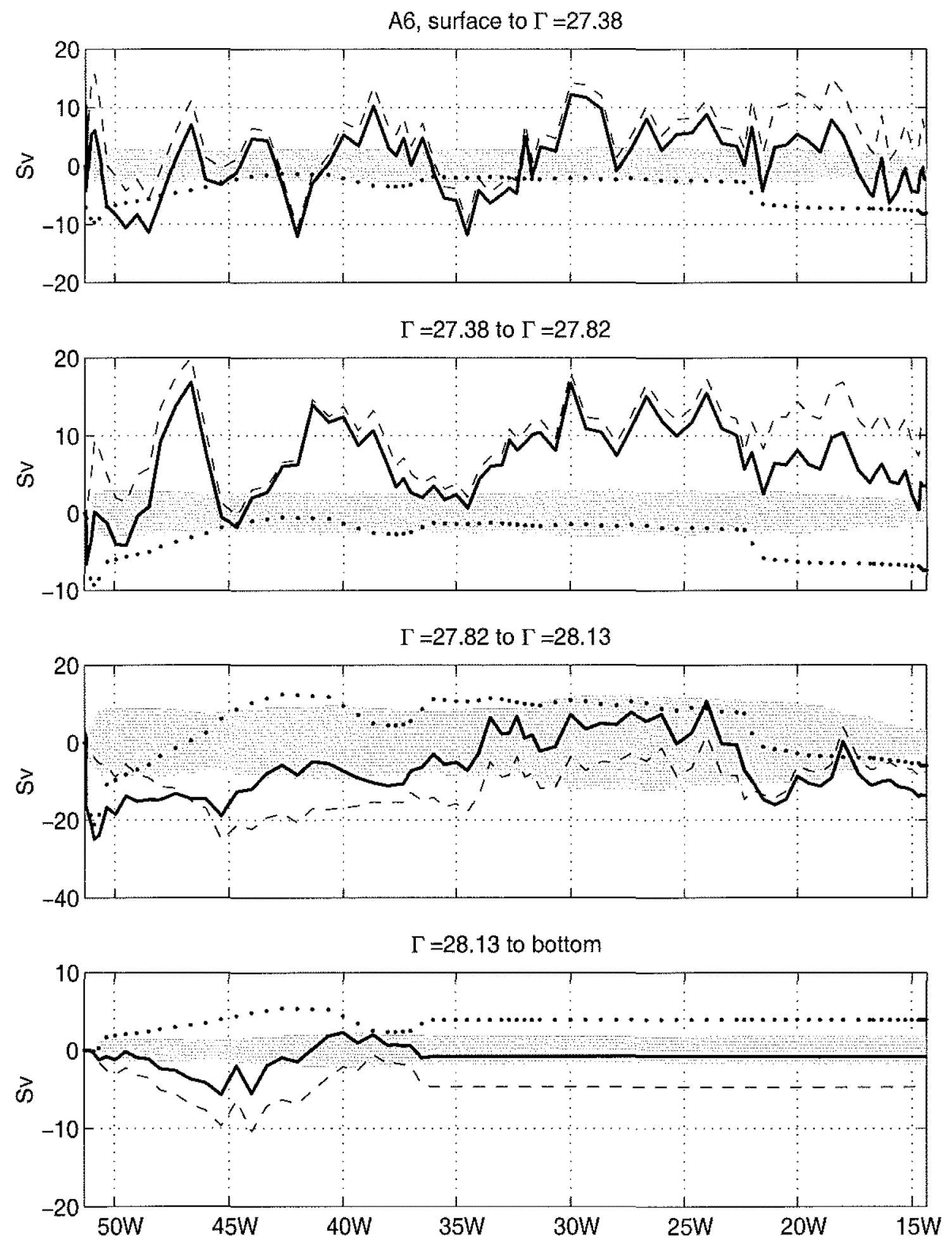

Figure D-30: Cumulative transports, Atlantic Ocean, A6 $\left(7.5^{\circ} \mathrm{N}\right)$. 


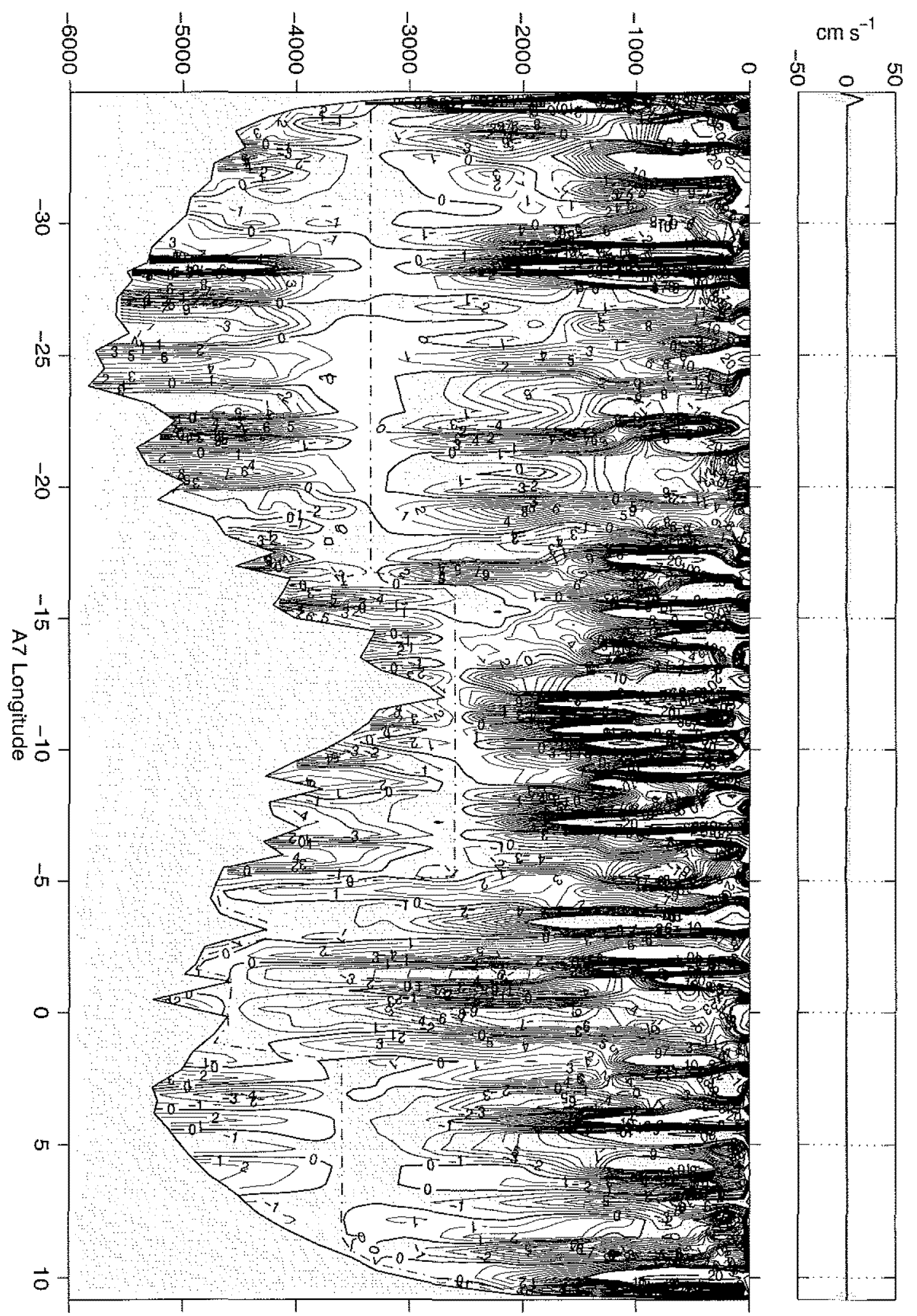

Figure D-31: Absolute velocities, Atlantic Ocean, A7 (4.5 ${ }^{\circ}$ ). 

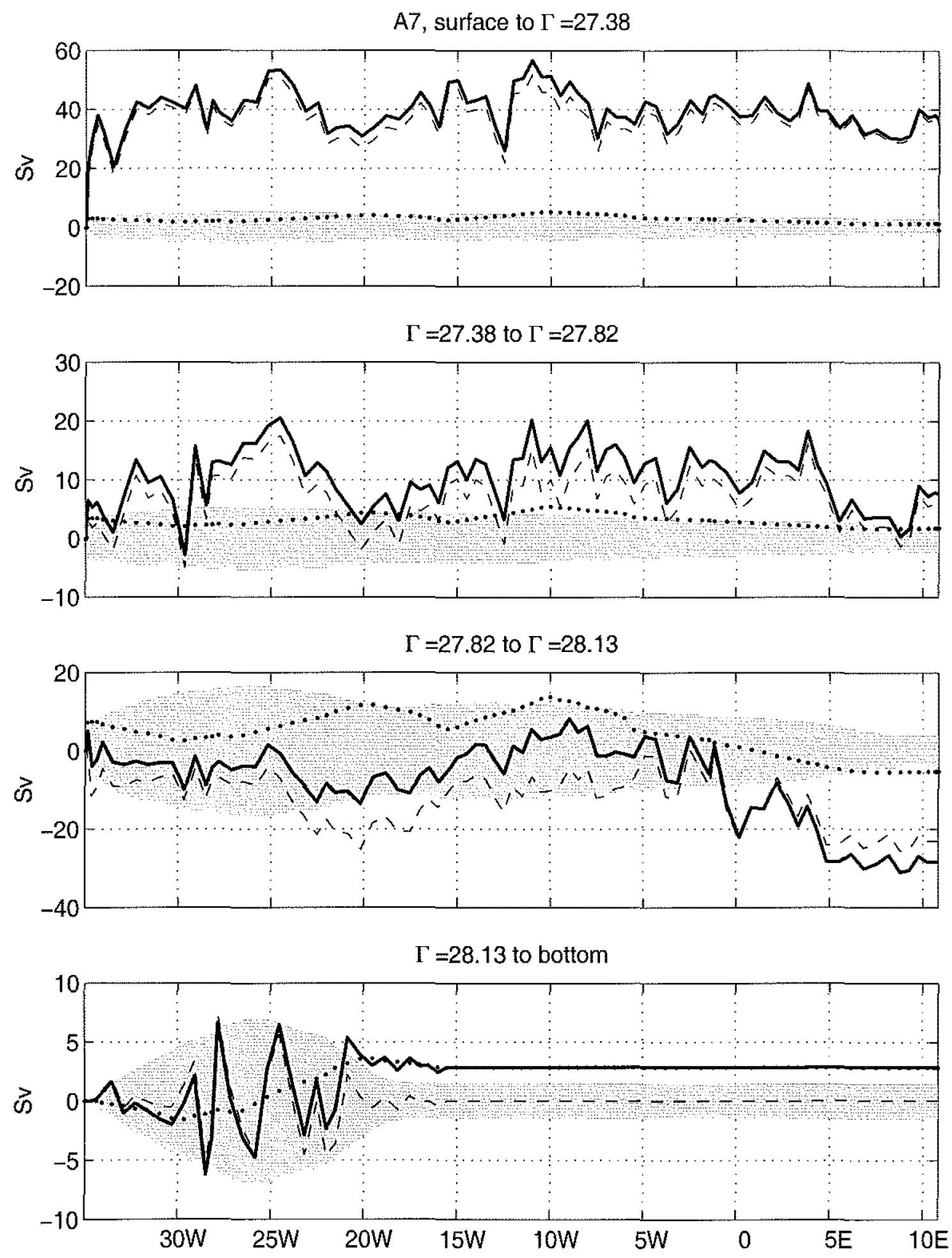

Figure D-32: Cumulative transports, Atlantic Ocean, A7 (4.5 $\left.5^{\circ}\right)$. 


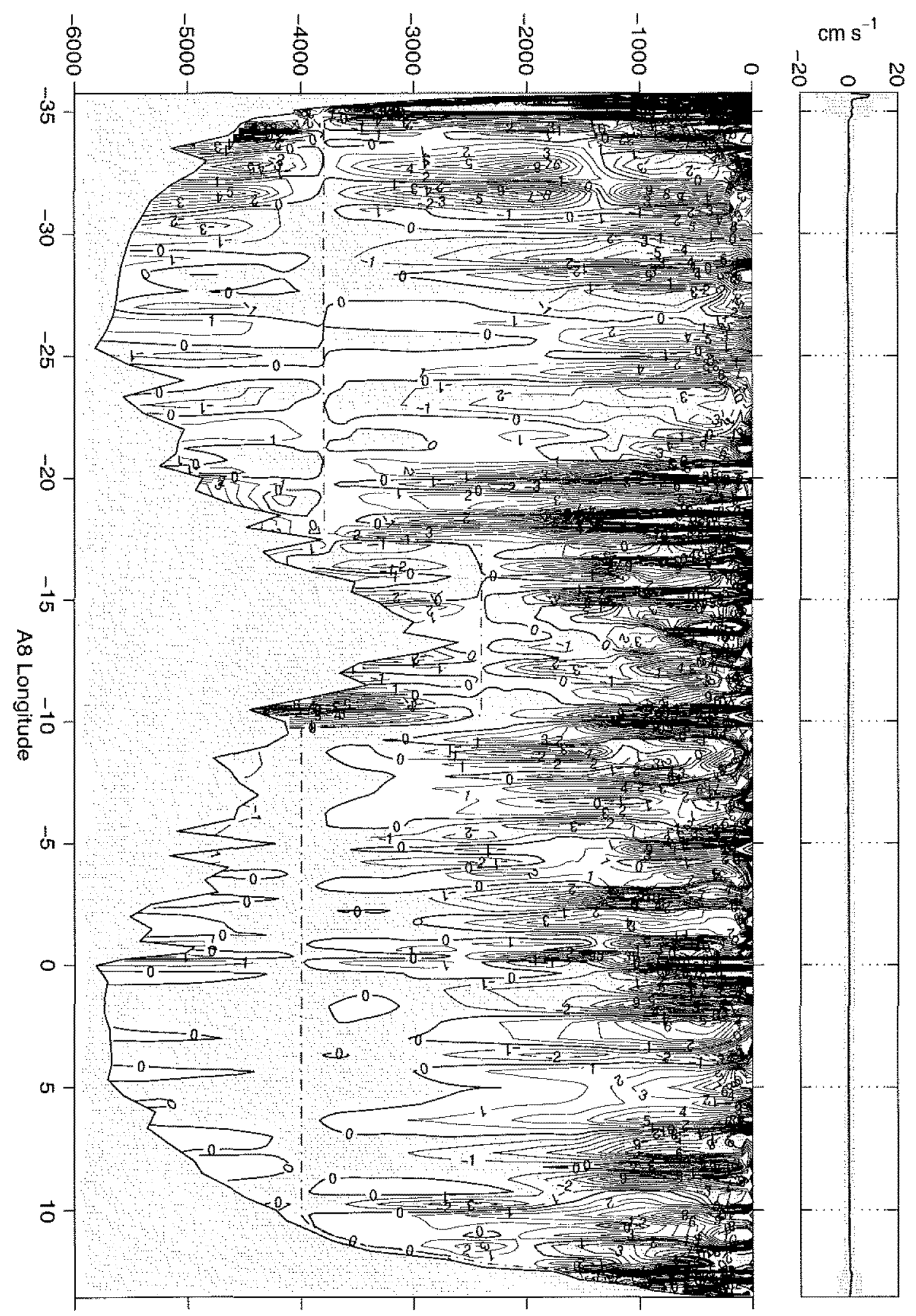

Figure D-33: Absolute velocities, Atlantic Ocean, A8 (11.4 $\left.{ }^{\circ} \mathrm{S}\right)$. 

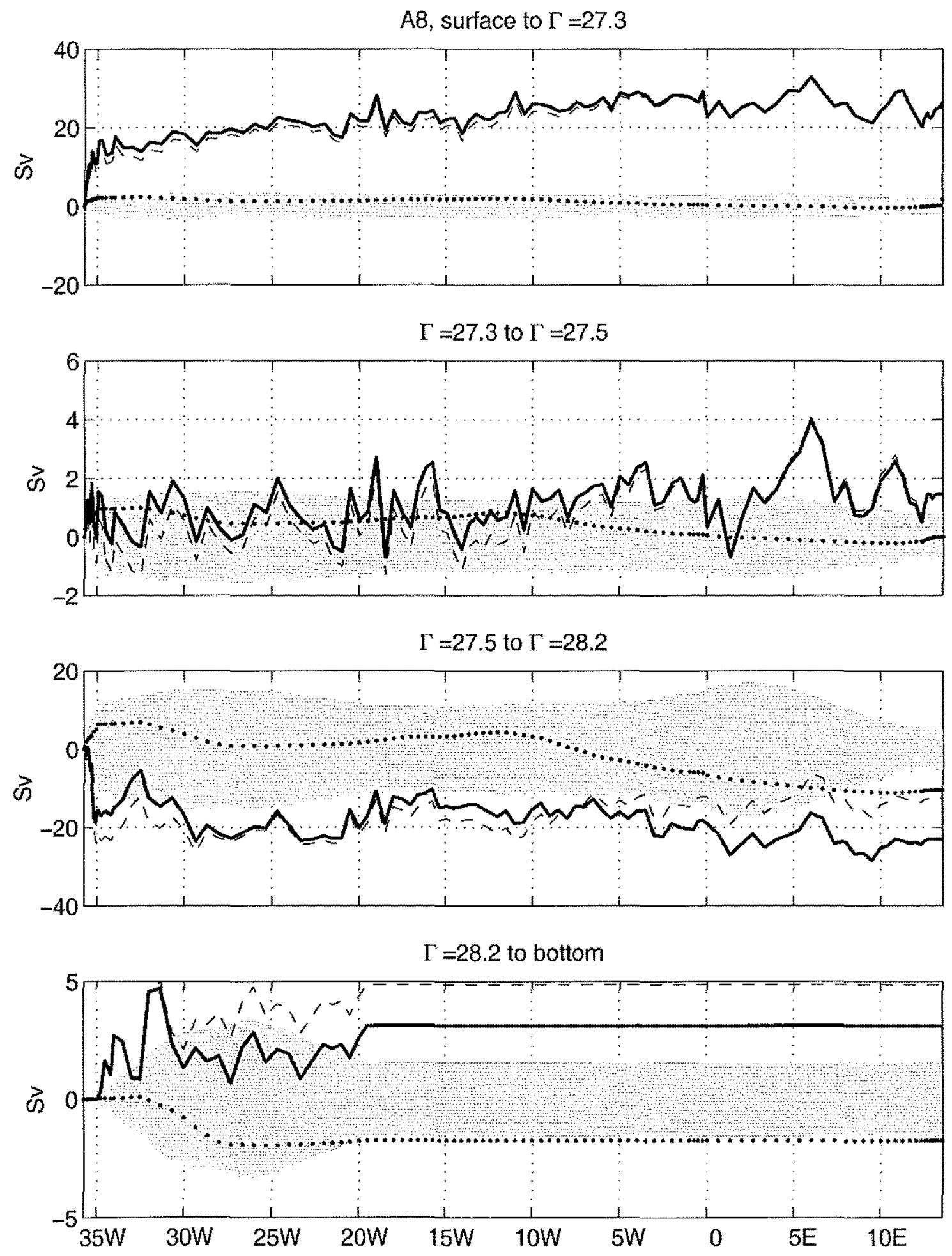

Figure D-34: Cumulative transports, Atlantic Ocean, A8 (11.4 $\left.{ }^{\circ} \mathrm{S}\right)$. 


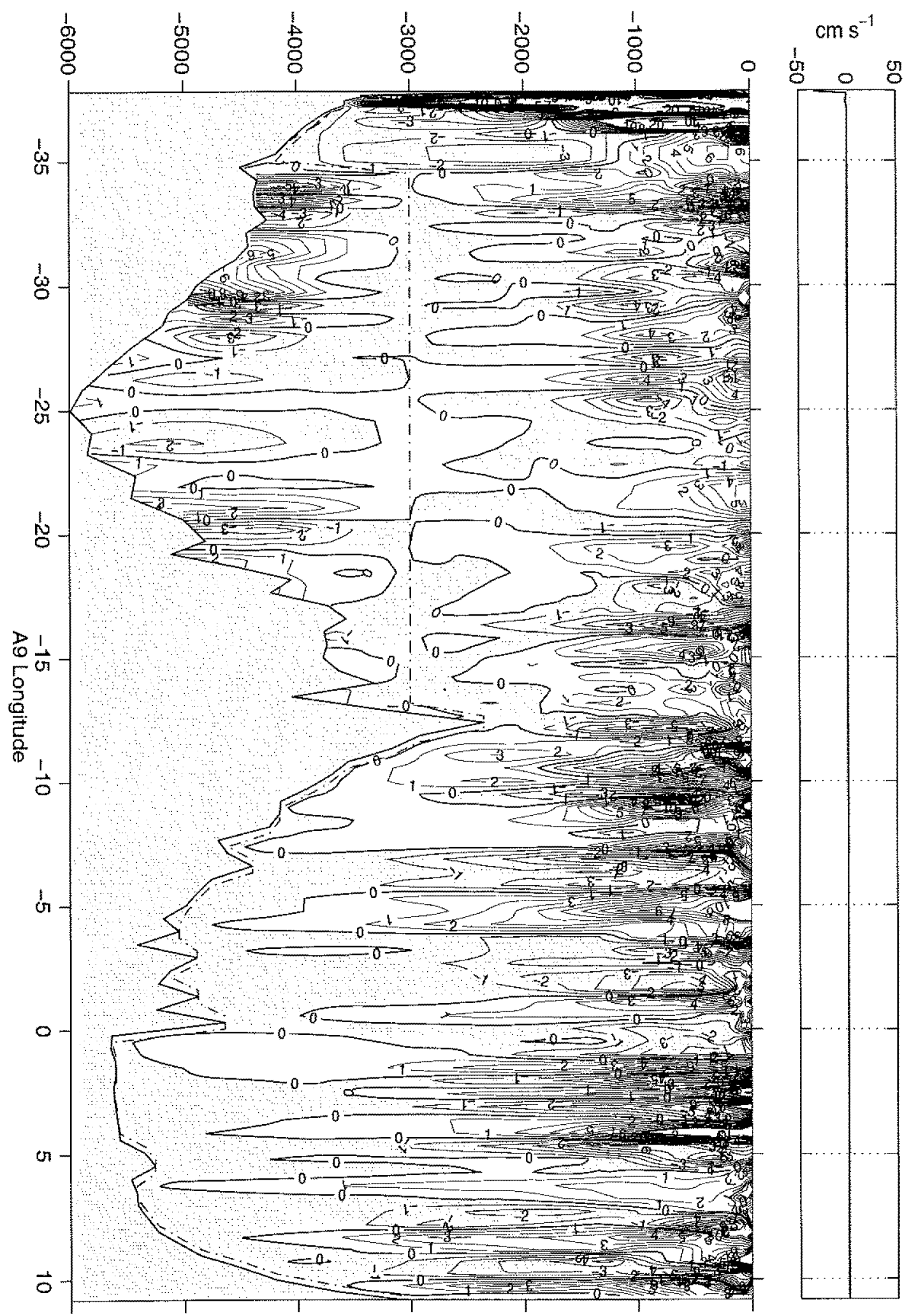

Figure D-35: Absolute velocities, Atlantic Ocean, A9 (195). 

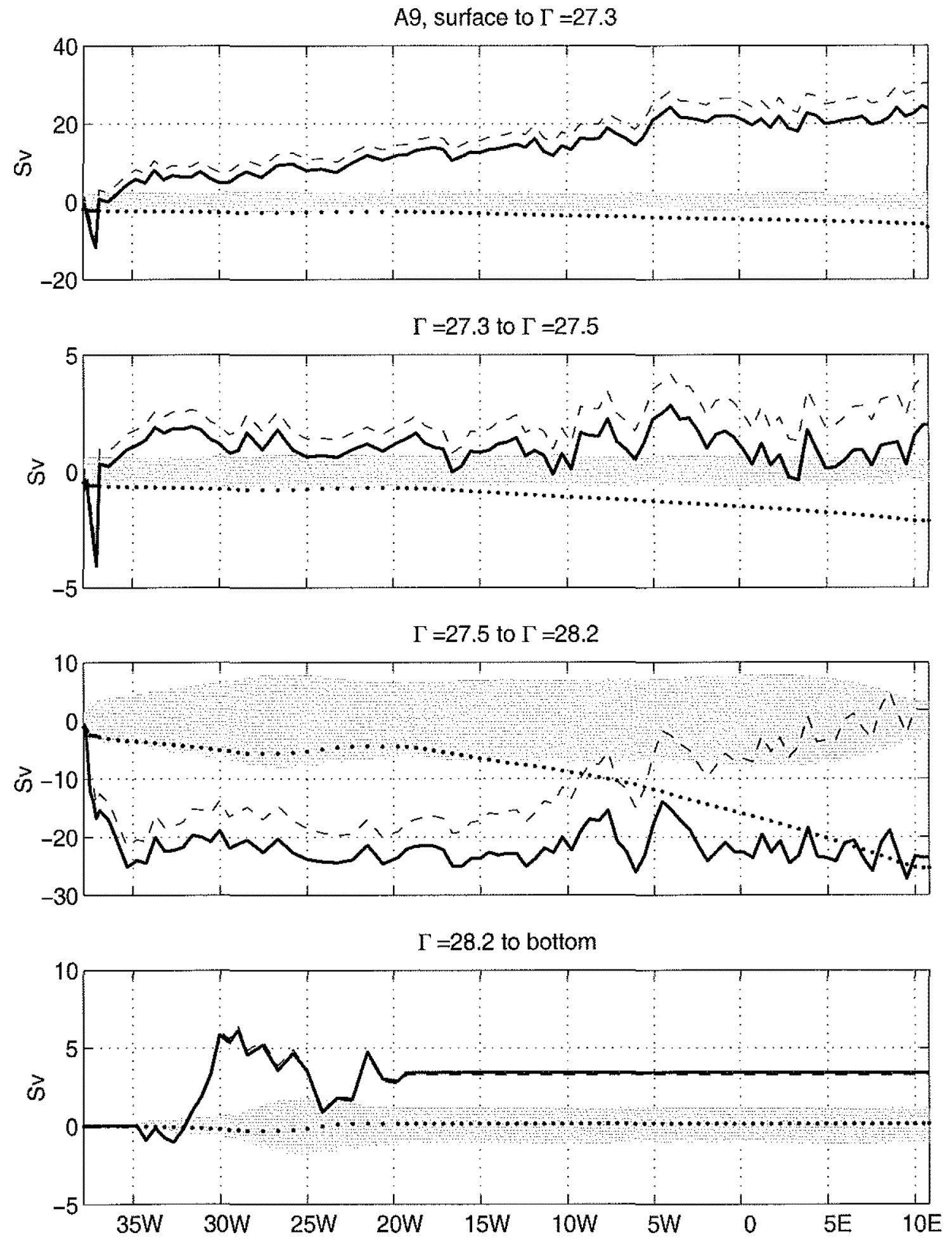

Figure D-36: Cumulative transports, Atlantic Ocean, A9 (19 $\left.{ }^{\circ} \mathrm{S}\right)$. 


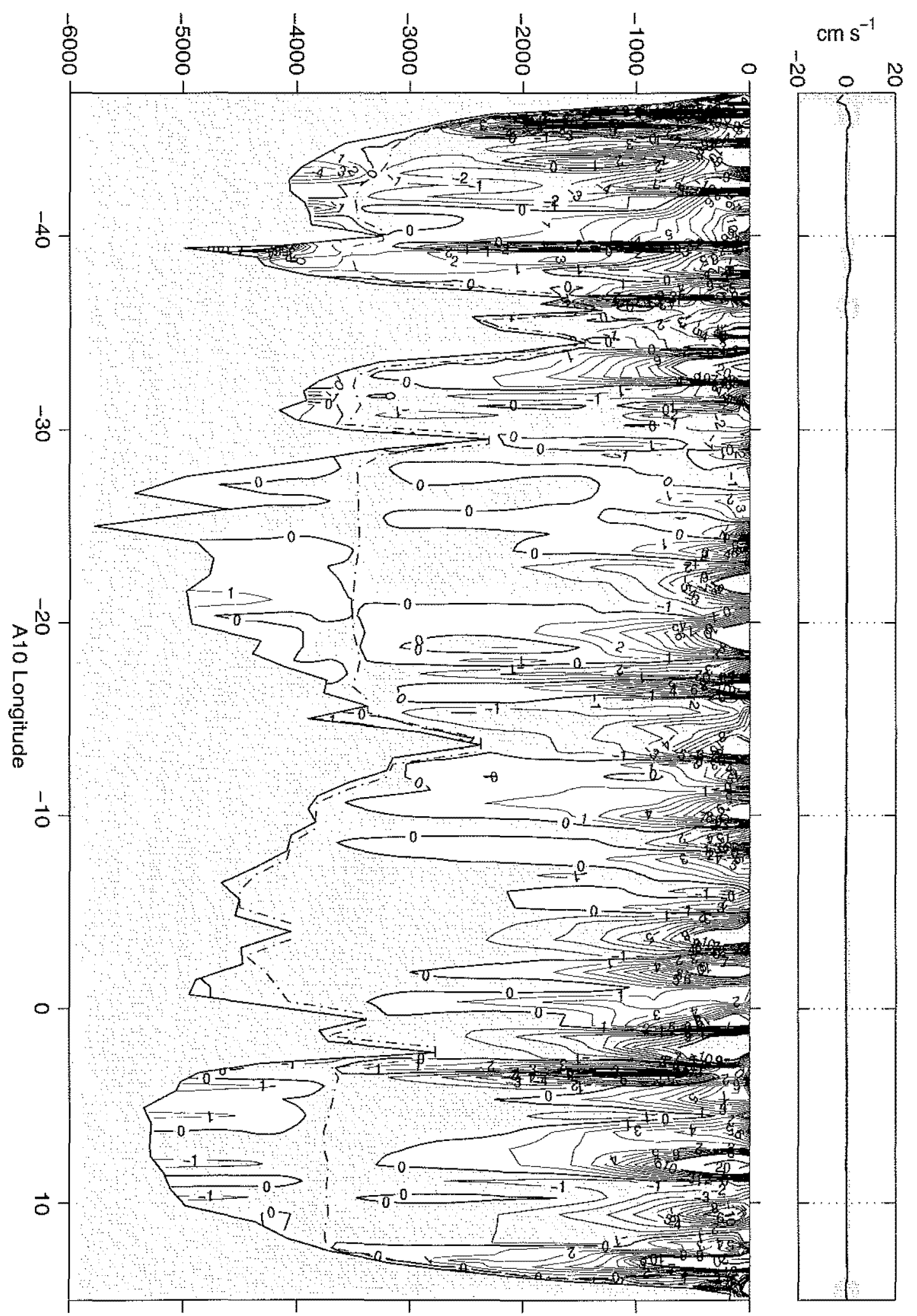

Figure D-37: Absolute velocities, Atlantic Ocean, A10 (30 $\mathrm{S})$. 

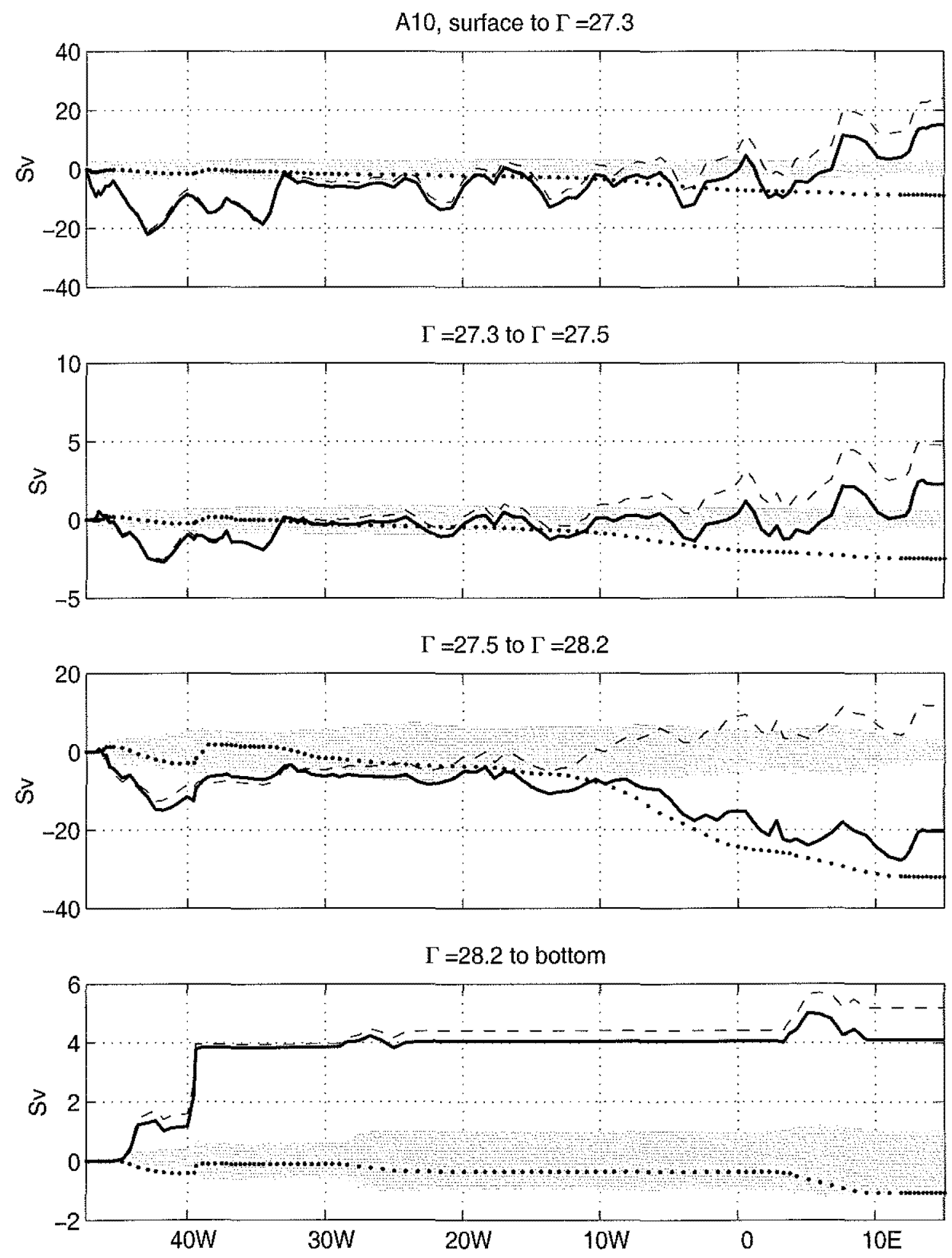

Figure D-38: Cumulative transports, Atlantic Ocean, A10 (30 S). 


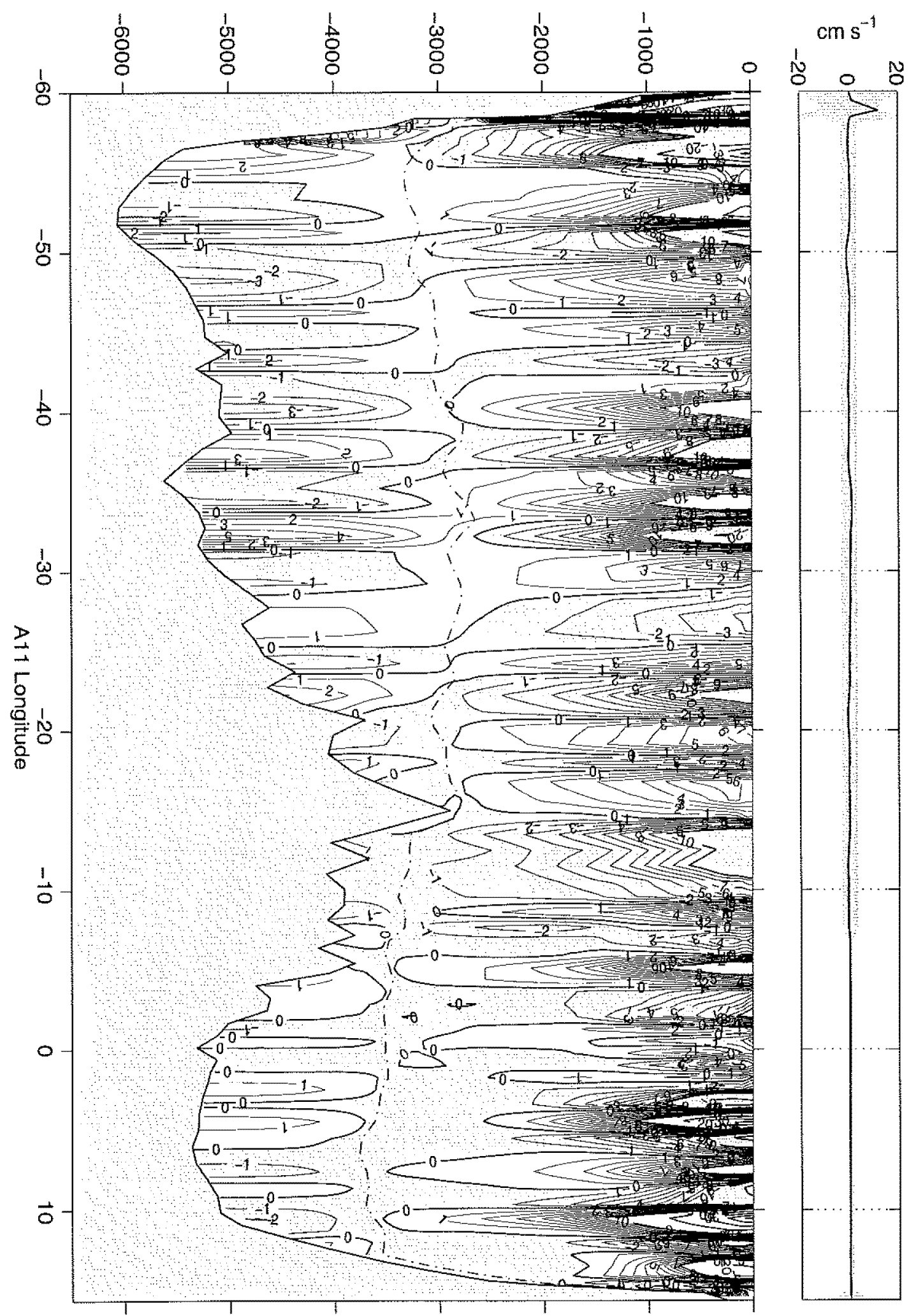

Figure D-39: Absolute velocities, Atlantic Ocean, A11 (45-30 $0^{\circ}$ ). 

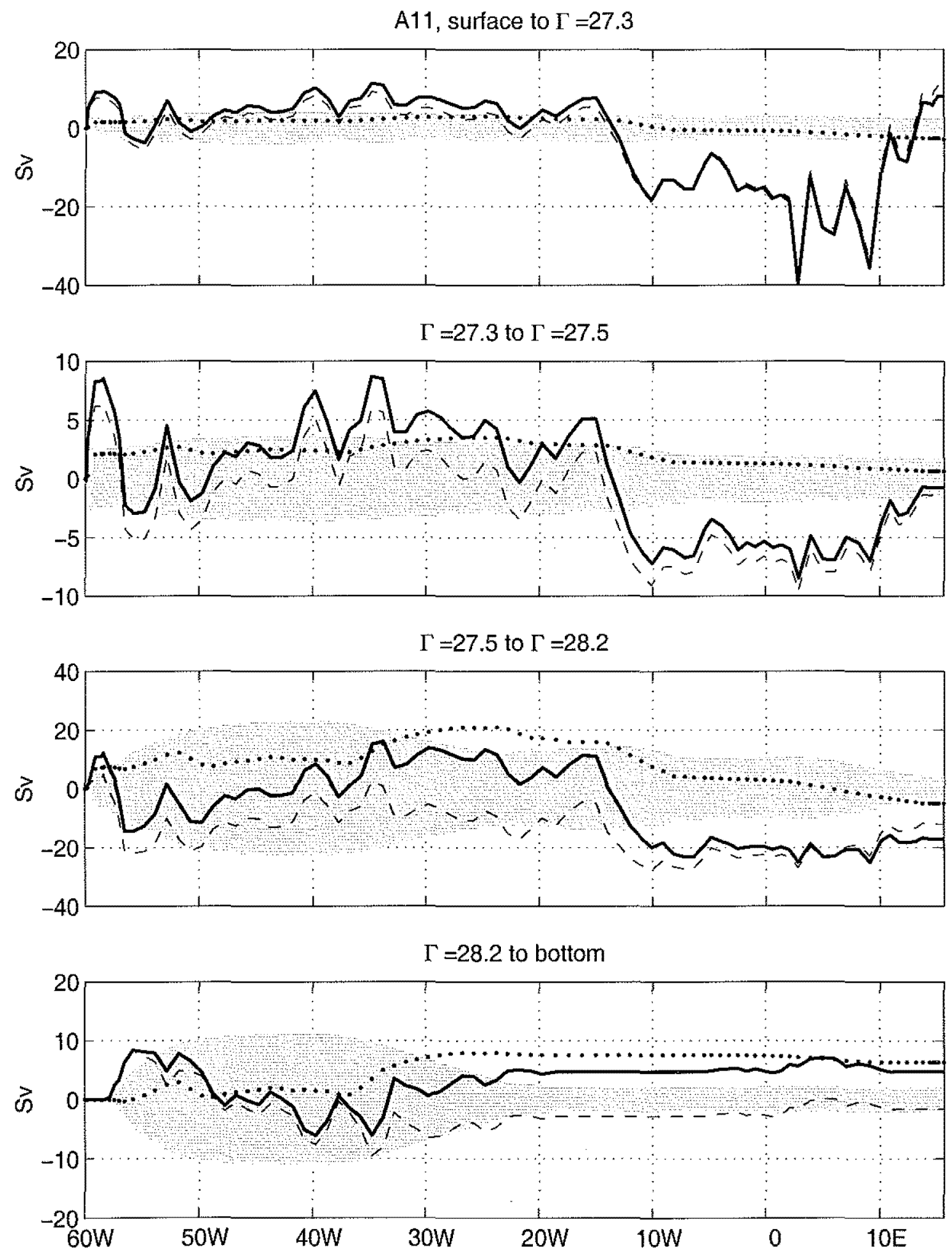

Figure D-40: Cumulative transports, Atlantic Ocean, A11 (45-30'S). 


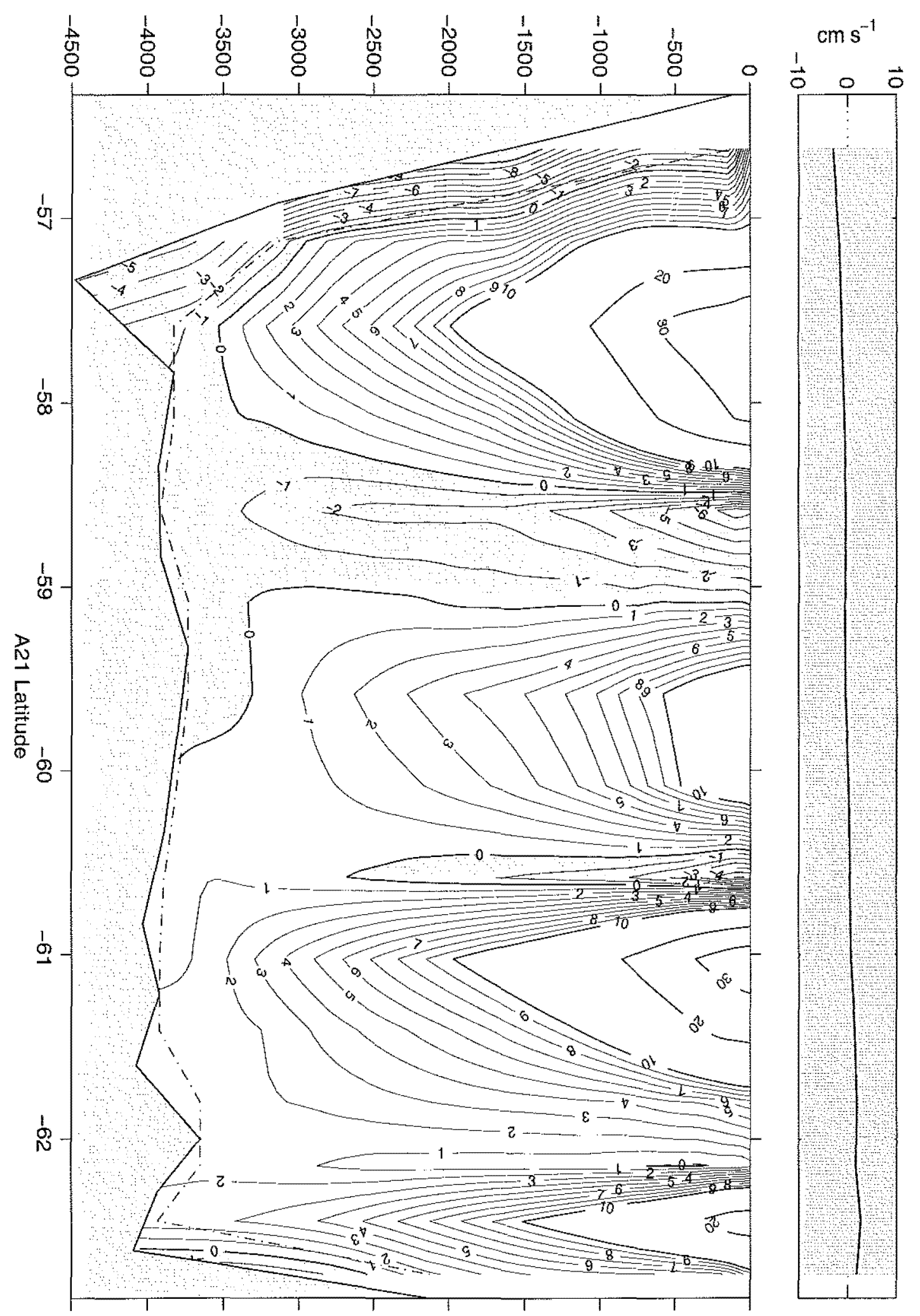

Figure D-41: Absolute velocities, Southern Ocean, A21 $\left(68^{\circ} \mathrm{W}\right)$. 

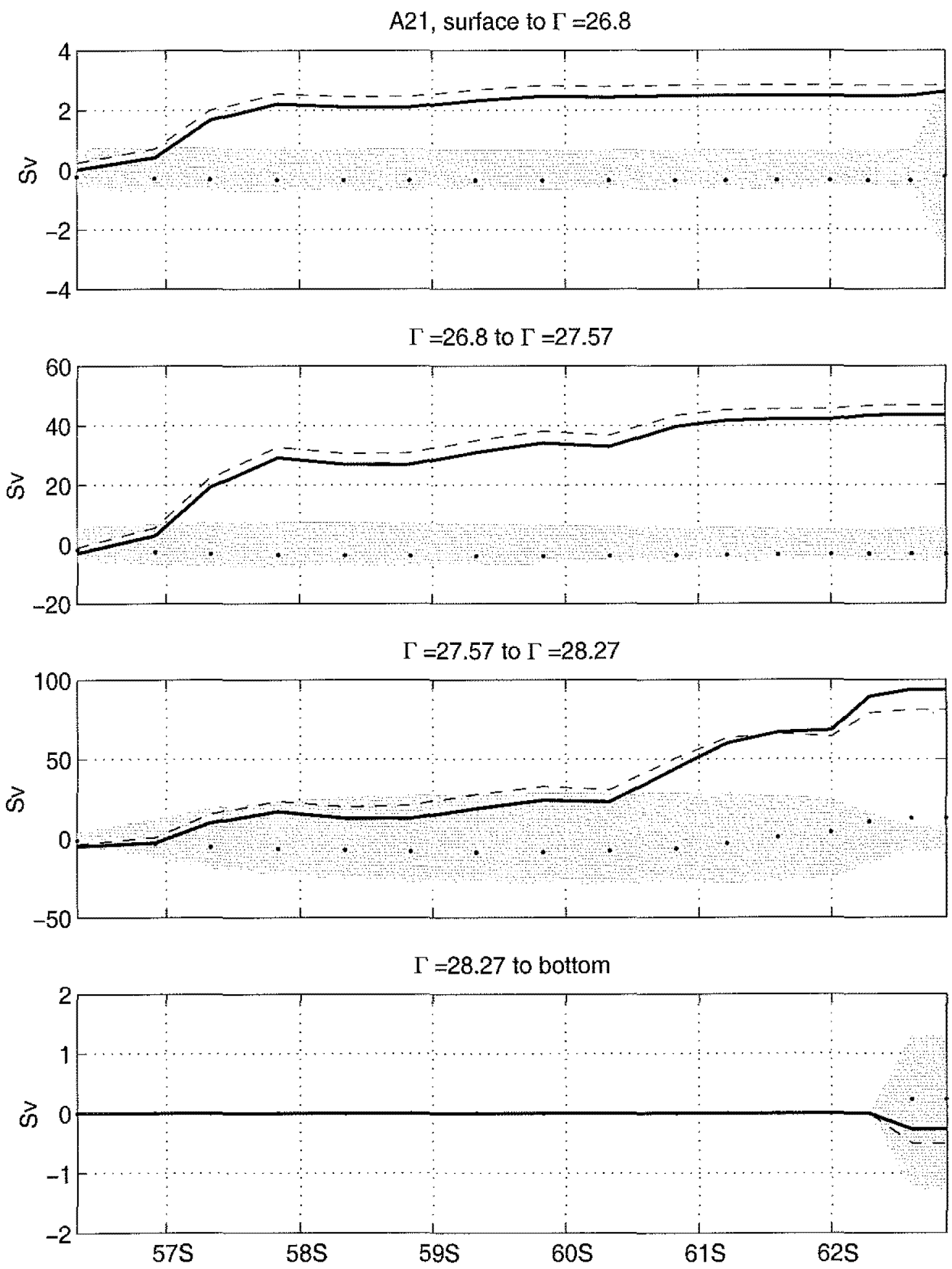

Figure D-42: Cumulative transports, Southern Ocean, A21 $\left(68^{\circ} \mathrm{W}\right)$. 


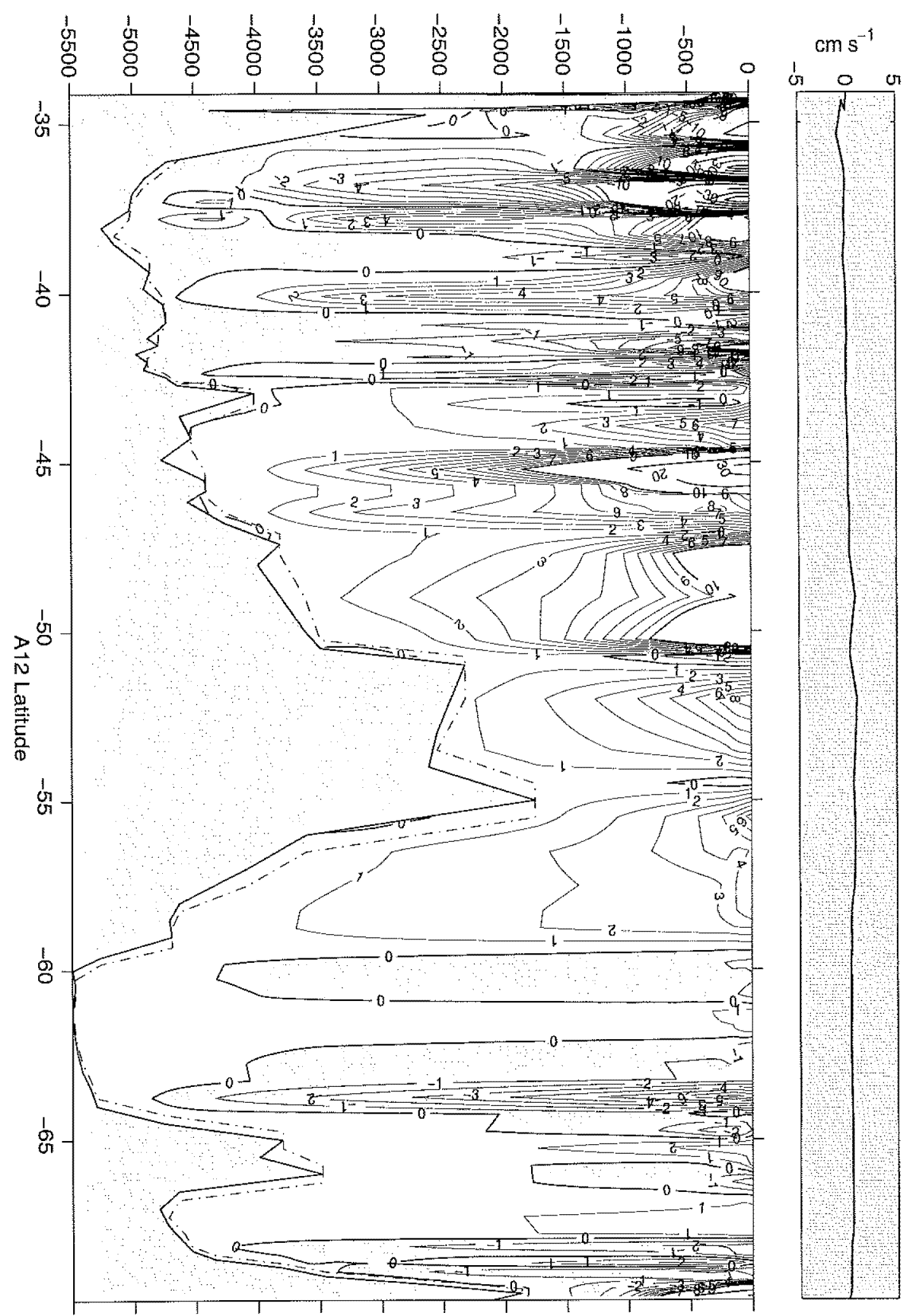

Figure D-43: Absolute velocities, Southern Ocean, A12 $\left(0^{\circ} \mathrm{E}\right)$. 

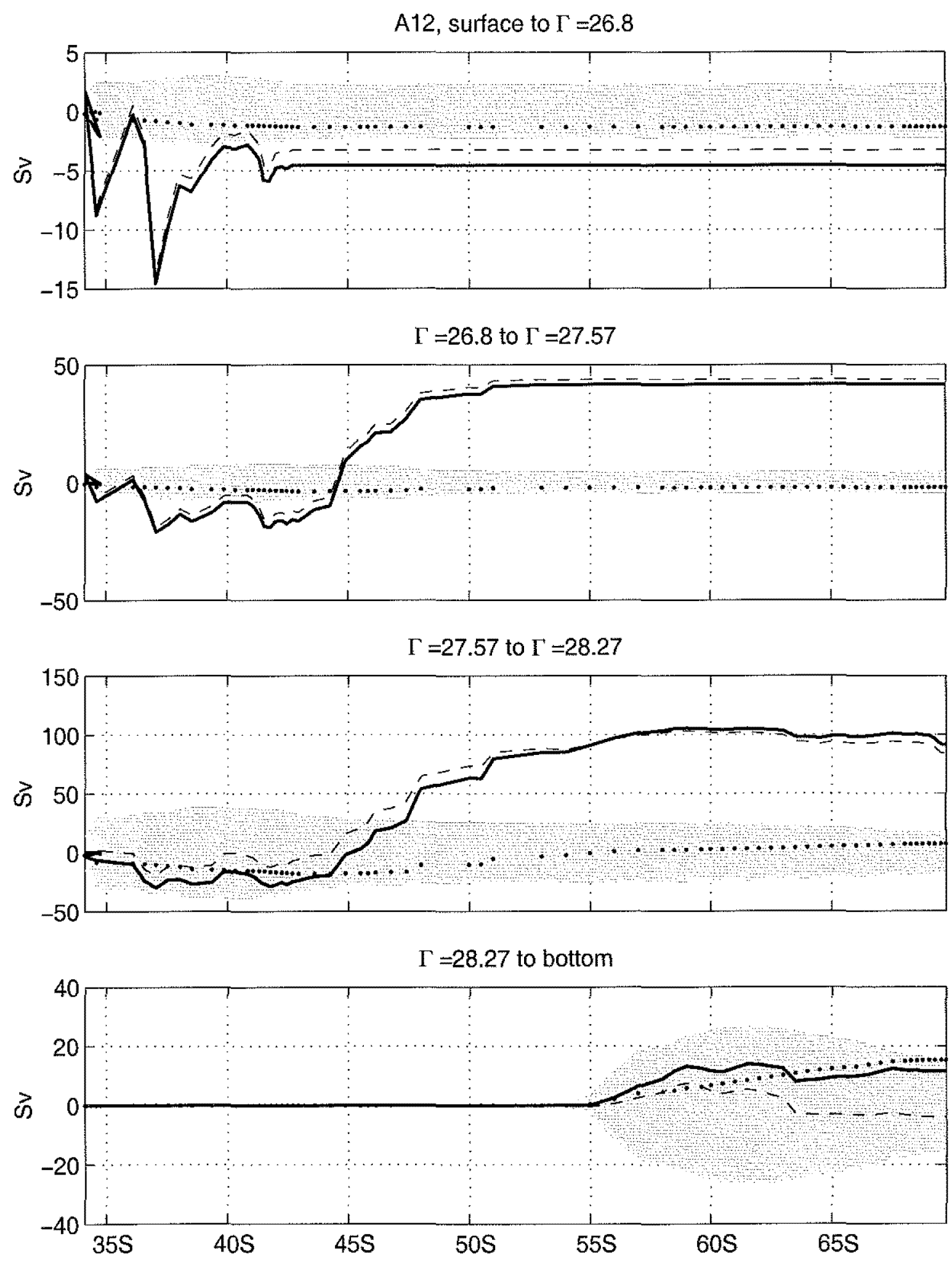

Figure D-44: Cumulative transports, Southern Ocean, A12 (0 $\left.0^{\circ} \mathrm{E}\right)$. 


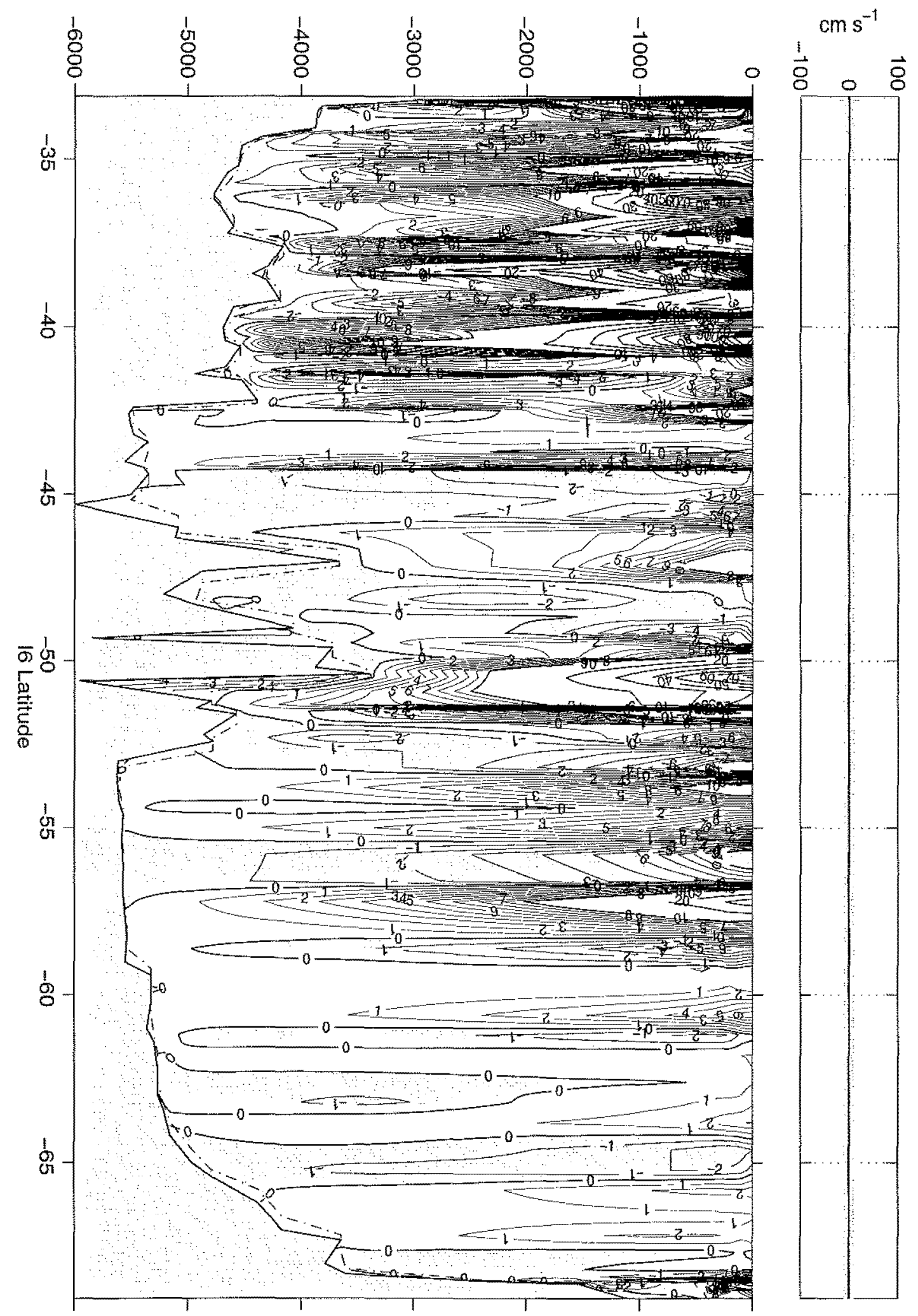

Figure D-45: Absolute velocities, Southern Ocean, I6 (30 E). 
16 , surface to $\Gamma=26.8$
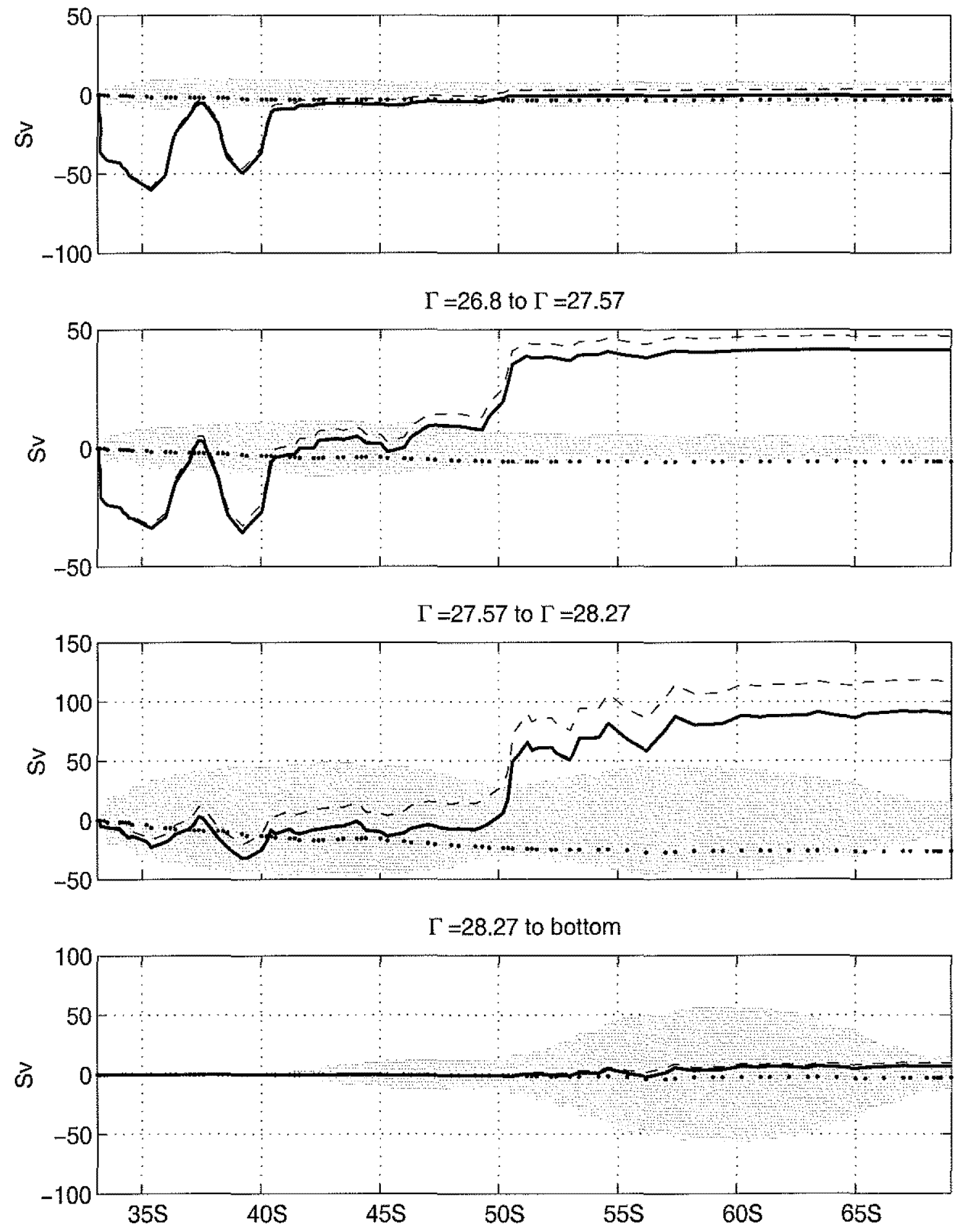

Figure D-46: Cumulative transports, Southern Ocean, $16\left(30^{\circ} \mathrm{E}\right)$. 


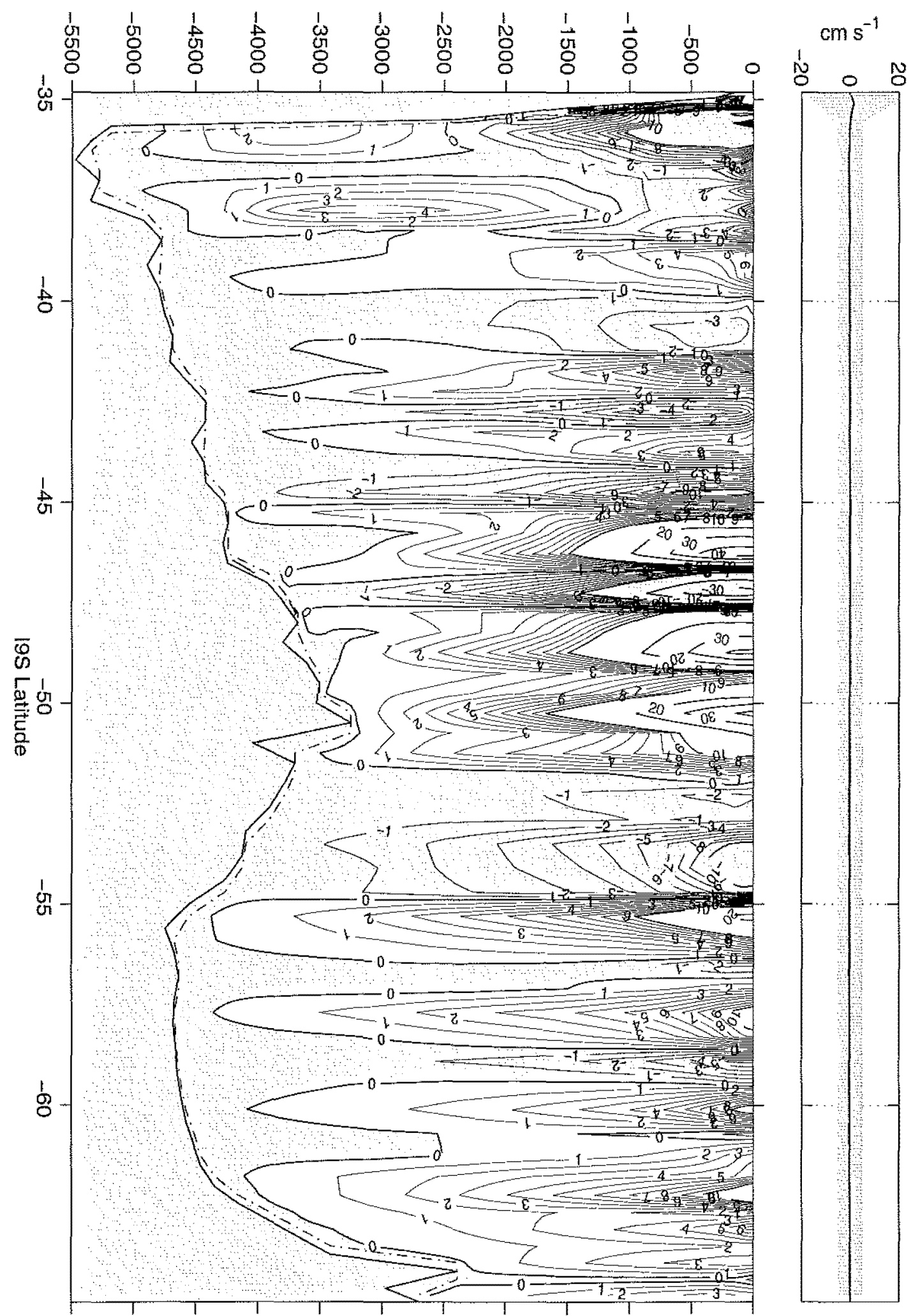

Figure D-47: Absolute velocities, Southern Ocean, I9S $\left(115^{\circ} \mathrm{E}\right)$. 

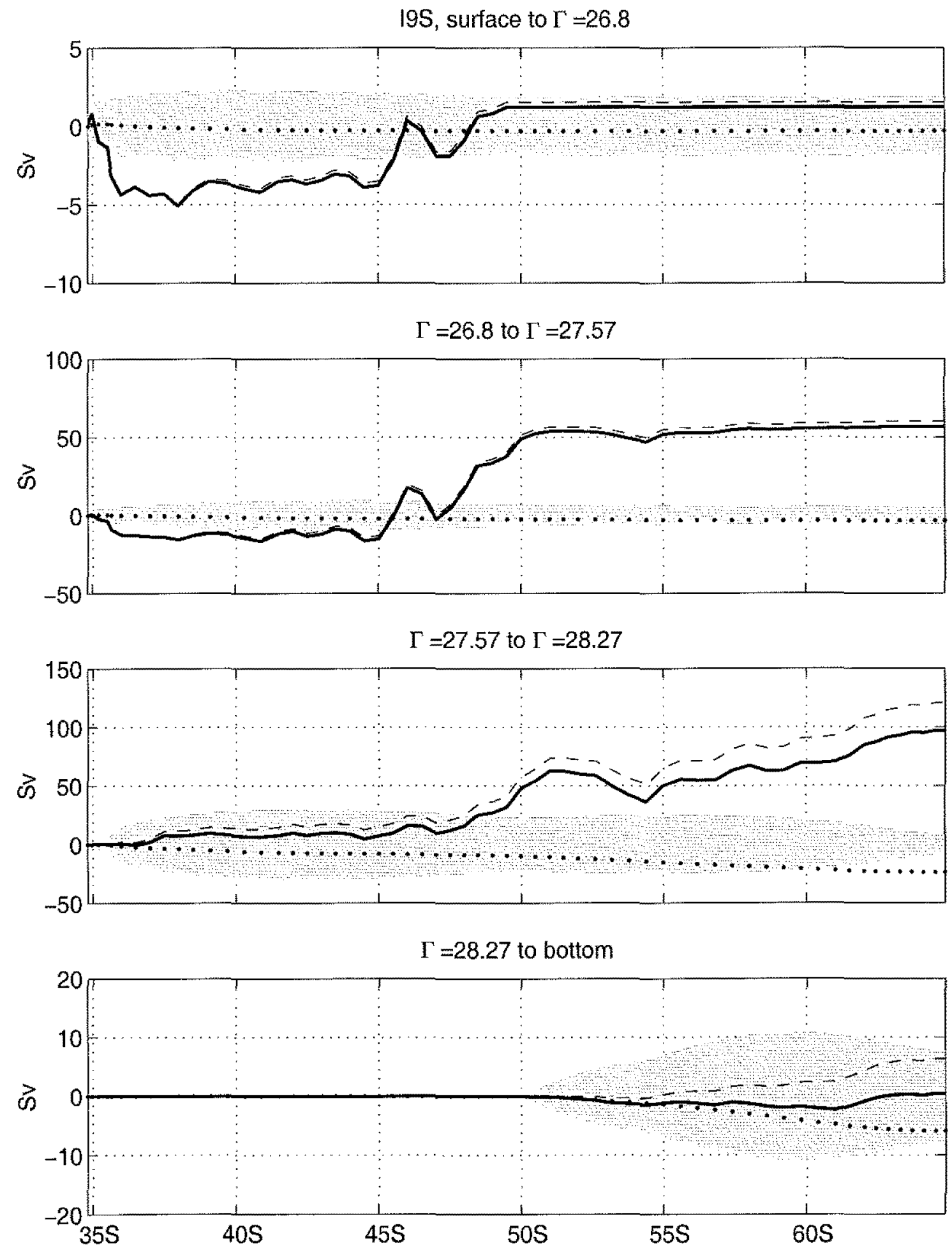

Figure D-48: Cumulative transports, Southern Ocean, $19 \mathrm{~S}\left(115^{\circ} \mathrm{E}\right)$. 


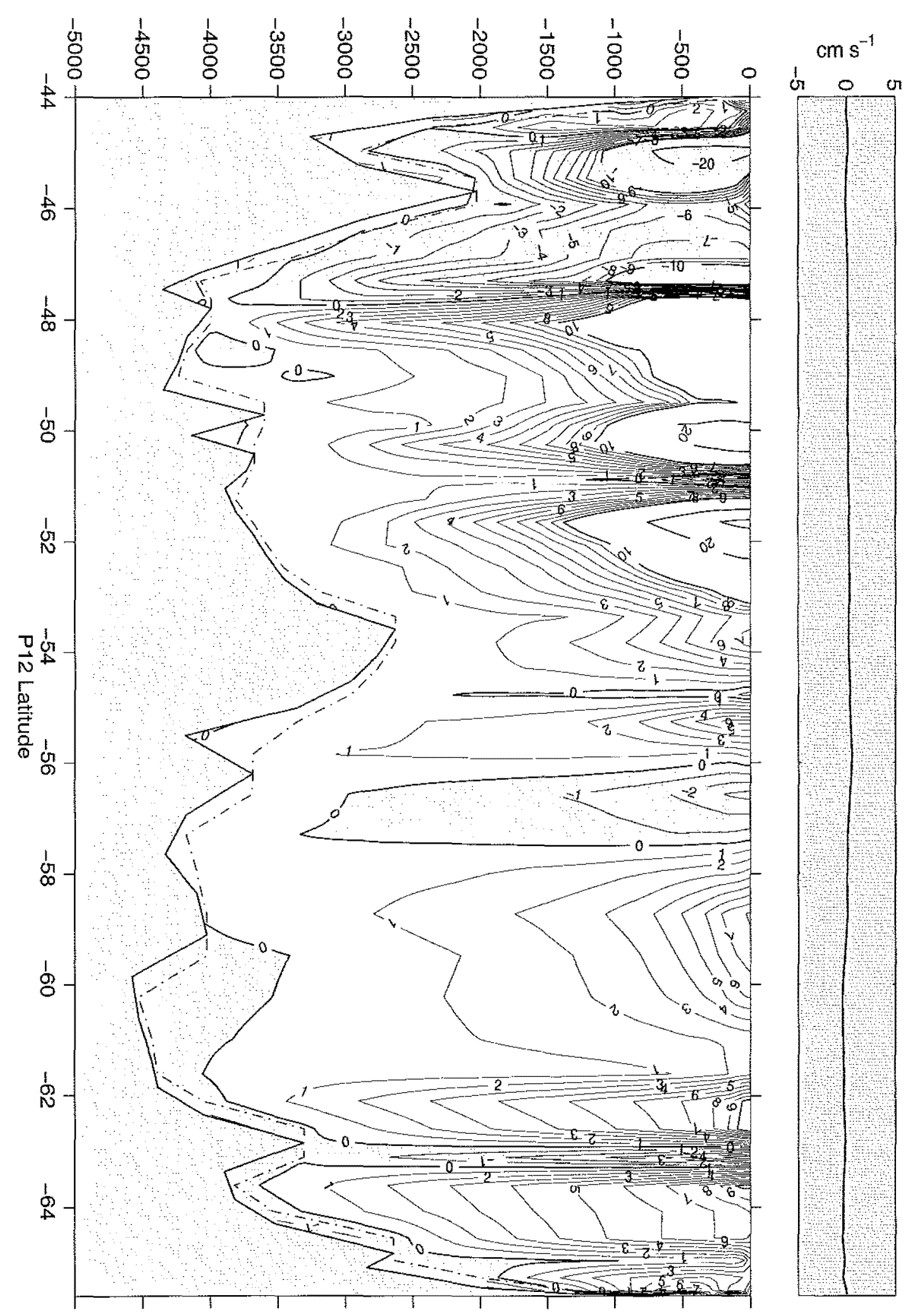

Figure D-49: Absolute velocities, Southern Ocean, P12 (140 E). 

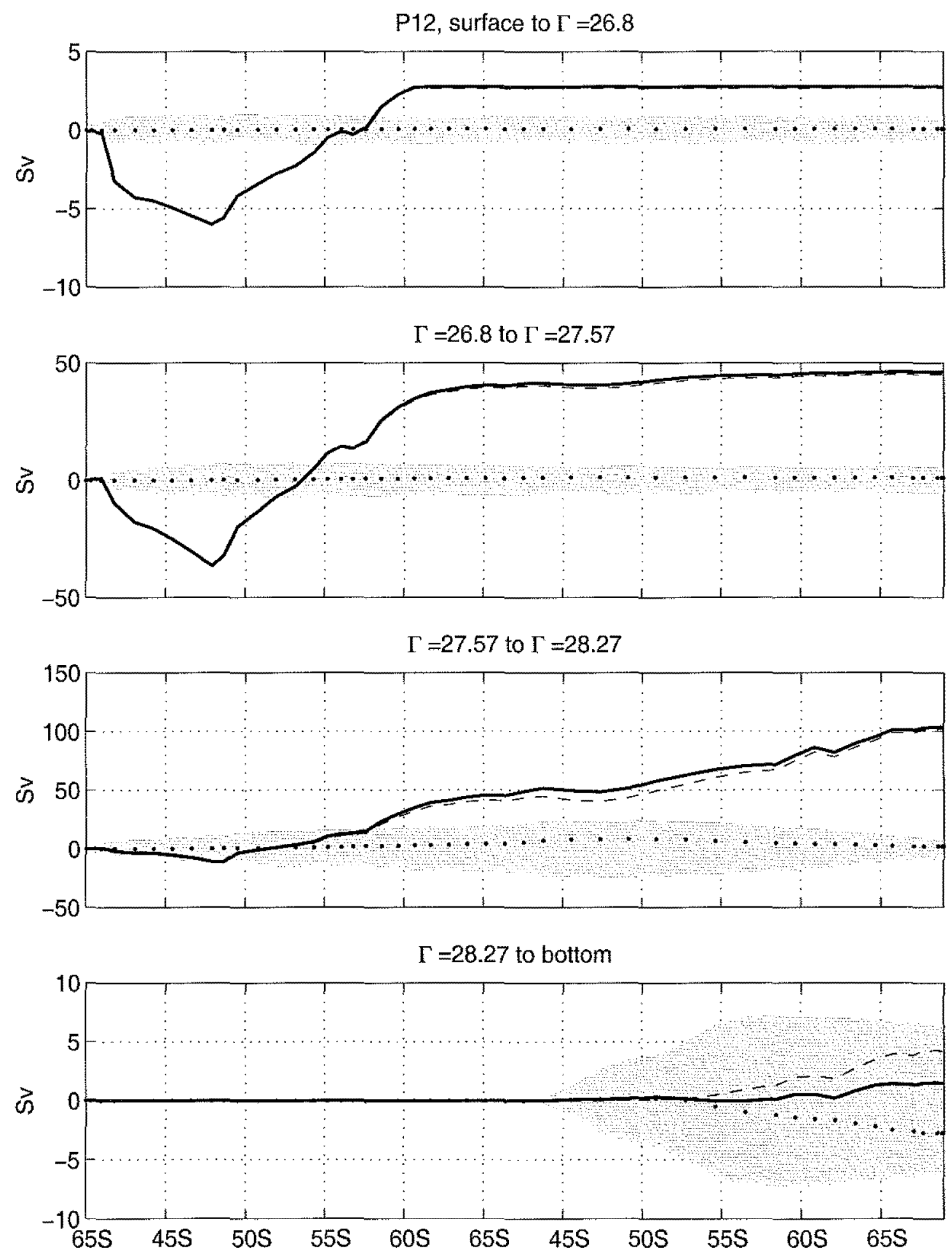

Figure D-50: Cumulative transports, Southern Ocean, $\mathrm{P} 12\left(140^{\circ} \mathrm{E}\right)$. 


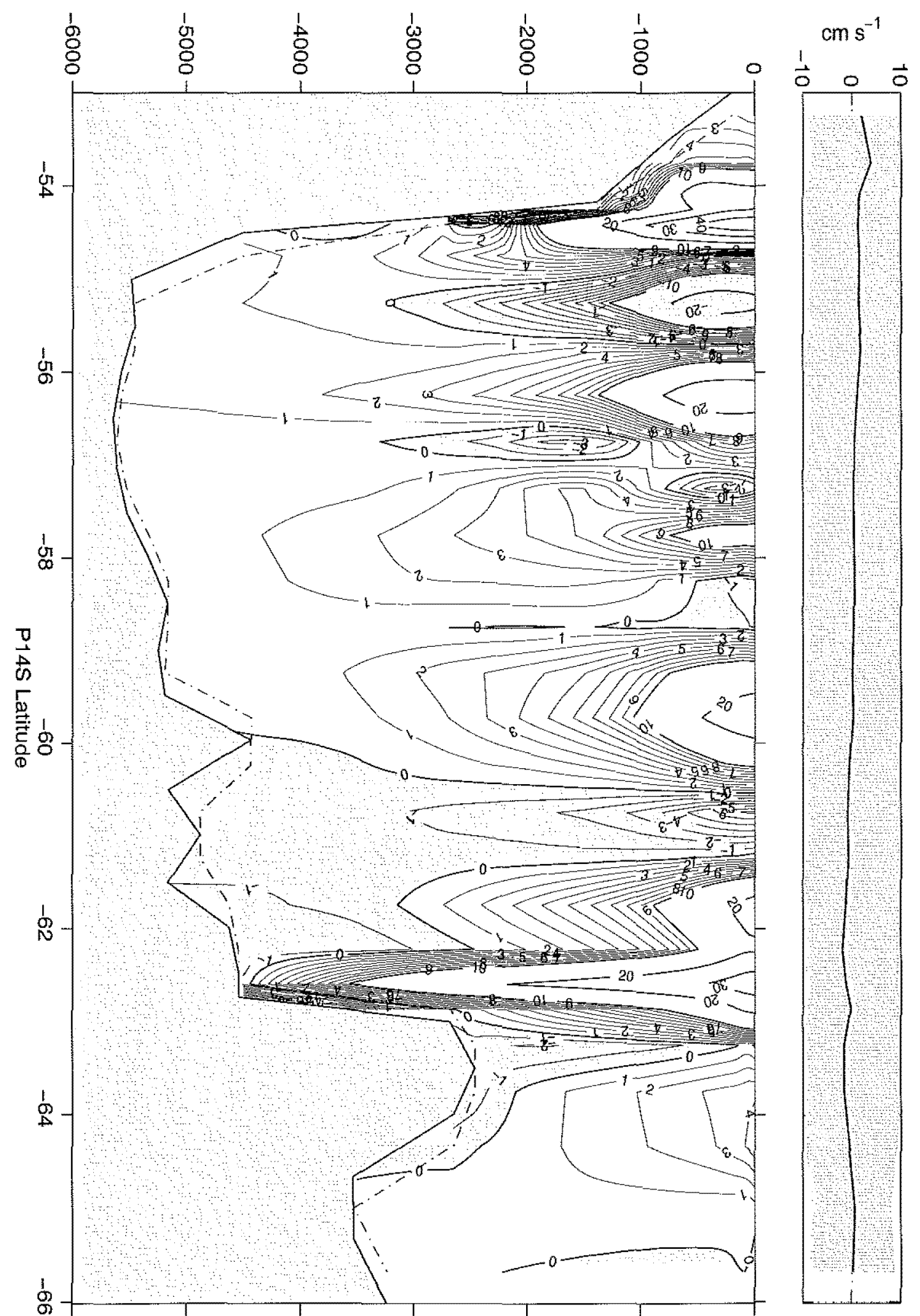

Figure D-51: Absolute velocities, Southern Ocean, P14S $\left(170^{\circ} \mathrm{W}\right)$. 

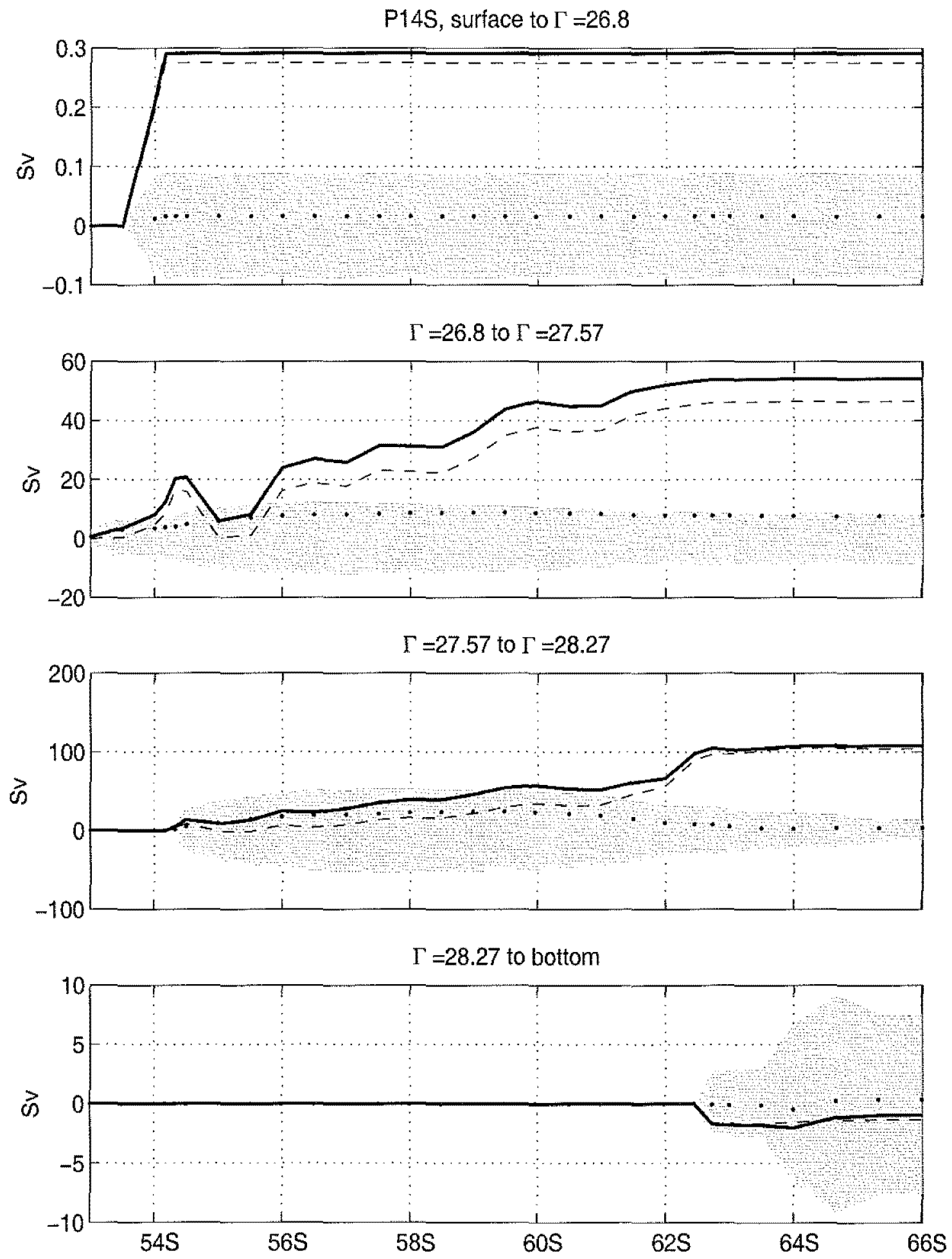

Figure D-52: Cumulative transports, Southern Ocean, P14S $\left(170^{\circ} \mathrm{W}\right)$. 
Appendix E

Standard solution property fluxes 


\begin{tabular}{|l|c|c|c|c|c|}
\hline Section & Temp. & Oxygen & Phosphate & Nitrate & Silica \\
\hline A2 & $0.6 \pm 0.1$ & $-1750 \pm 500$ & $1.1 \pm 2.5$ & $8 \pm 35$ & $-130 \pm 50$ \\
\hline A5+Fl. C. & $1.3 \pm 0.15$ & $-2070 \pm 600$ & $-7.6 \pm 3.6$ & $-55 \pm 55$ & $-220 \pm 80$ \\
\hline Fl. Current & $2.4 \pm 0.07$ & $5260 \pm 150$ & $14 \pm 1.2$ & $260 \pm 20$ & $154 \pm 13$ \\
\hline A6 & $0.7 \pm 0.3$ & $-1430 \pm 950$ & $-1.0 \pm 7$ & $-75 \pm 120$ & $-165 \pm 107$ \\
\hline A7 & $1.0 \pm 0.55$ & $-138 \pm 1568$ & $-11 \pm 10$ & $-210 \pm 150$ & $-190 \pm 170$ \\
\hline A8 & $0.9 \pm 0.4$ & $-1380 \pm 1250$ & $N / A$ & $-180 \pm 140$ & $-230 \pm 154$ \\
\hline A9 & $0.8 \pm 0.2$ & $-1590 \pm 700$ & $N / A$ & $-60 \pm 80$ & $-134 \pm 107$ \\
\hline A10 & $0.35 \pm 0.15$ & $430 \pm 680$ & $-4 \pm 5$ & $-70 \pm 80$ & $-180 \pm 140$ \\
\hline A11 & $0.66 \pm 0.12$ & $-52 \pm 710$ & $-9.0 \pm 6$ & $-240 \pm 90$ & $-107 \pm 190$ \\
\hline A12 & $0.75 \pm 0.23$ & $32175 \pm 1900$ & $294 \pm 17$ & $4304 \pm 245$ & $9800 \pm 900$ \\
\hline A21 & $1.30 \pm 0.14$ & $31385 \pm 1230$ & $302 \pm 13$ & $4340 \pm 190$ & $9730 \pm 880$ \\
\hline I6 & $0.90 \pm 0.6$ & $31555 \pm 1900$ & $295 \pm 27$ & $4095 \pm 340$ & $9810 \pm 920$ \\
\hline I5 & $-1.50 \pm 0.23$ & $-1820 \pm 1090$ & $-4.3 \pm 9$ & $-85 \pm 130$ & $250 \pm 375$ \\
\hline I9S & $1.72 \pm 0.3$ & $34248 \pm 1840$ & $320 \pm 18$ & $4620 \pm 264$ & $9547 \pm 920$ \\
\hline P12 & $1.42 \pm 0.24$ & $34345 \pm 1800$ & $330 \pm 20$ & $4700 \pm 266$ & $9520 \pm 920$ \\
\hline P14S & $1.64 \pm 0.26$ & $35551 \pm 3260$ & $326 \pm 30$ & $4815 \pm 440$ & $10330 \pm 1300$ \\
\hline P6 & $0.6 \pm 0.2$ & $4725 \pm 1000$ & $14 \pm 10$ & $186 \pm 136$ & $-275 \pm 380$ \\
\hline P21 & $0.21 \pm 0.46$ & $3240 \pm 1100$ & $25 \pm 12$ & $270 \pm 170$ & $-130 \pm 416$ \\
\hline P3 & $0.53 \pm 0.24$ & $-1410 \pm 680$ & $0.8 \pm 8$ & $-1 \pm 100$ & $46 \pm 344$ \\
\hline JADE 89-92 & $-1.36 \pm 0.14$ & $-2248 \pm 407$ & $-12 \pm 6$ & $-155 \pm 80$ & $-390 \pm 190$ \\
\hline P1 & $0.0 \pm 0.05$ & $-672 \pm 450$ & $4.7 \pm 3$ & $76 \pm 44$ & $66 \pm 150$ \\
\hline I4 & $-0.77 \pm 0.2$ & $-2114 \pm 1126$ & $-19 \pm 11$ & $-261 \pm 150$ & $-311 \pm 311$ \\
\hline I3+I4 & $-1.79 \pm 0.44$ & $-1416 \pm 1210$ & $-19 \pm 10$ & $-240 \pm 150$ & $33 \pm 440$ \\
\hline I2W & $-0.77 \pm 0.8$ & $-3500 \pm 1670$ & $-10 \pm 16$ & $-123 \pm 218$ & $-178 \pm 516$ \\
\hline I2W+I2+I10 & $-1.57 \pm 0.8$ & $-1758 \pm 1750$ & $-28 \pm 15$ & $-400 \pm 210$ & $-260 \pm 530$ \\
\hline & & & & & \\
\hline
\end{tabular}

Table E.1: Standard solution fluxes of temperature (expressed in PW on the Celsius scale), oxygen, nitrate and phosphate $(\mathrm{kmol} / \mathrm{s})$. Positive is northward or eastward. Fl. C. stands for Florida Current. 


\section{References}

Anderson, L. A., and J. L. Sarmiento, Redfield ratios of remineralization determined by nutrient data analysis, Global Biogeochemical Cycles, 8(1), 65-85, 1994.

Antoine, D., J.-M. André, and A. Morel, Oceanic primary production 2. Estimation at global scale from satellite chlorophyll, Global Biogeochemical Cycles, 10(1), 57-69, 1996.

Arbic, B. K., and W. B. Owens, Climatic warming of Atlantic Intermediate Waters, in preparation, 1999.

Archer, D., M. Lyle, K. Rodgers, and P. Frolich, What controls opal preservation in tropical deep-sea sediments, Paleoceanography, 8(1), 7-21, 1993.

Arhan, M., H. Mercier, B. Bourles, and Y. Gouriou, Two hydrographic sections across the Atlantic at $7^{\circ} 30 \mathrm{~N}$ and $4^{\circ} 30 \mathrm{~S}$, Deep-Sea Res., in press, 1998.

Aston, S., S. Calvert, D. Hurd, R. Macdonald, F. Mackenzie, C. Spencer, and R. Wollast, Silicon Geochemistry and Biogeochemistry. Academic Press, 1983.

Banks, H., J. Bullister, S. Bacon, H. Bryden, and J. Rennell, The deep western boundary current at $17^{\circ} \mathrm{S}$ in the Pacific Ocean, Intl. WOCE News., (19), 3-5, 1995.

Banse, K., New views on the degradation and disposition of organic particles collected by sediment traps in the open sea, Deep-Sea Res., 37, 1177-1195, 1990.

Baumgartner, A., and E. Reichel, The world water balance. Elsevier Science, New York, 1975.

Beal, L. M., and H. L. Bryden, Observation of an Agulhas undercurrent, Deep-Sea Res., 44(I), 1715-1724, 1997.

Bohren, C. F., and B. A. Albrecht, Atmospheric Thermodynamics. Oxford, New York, 402pp., 1998.

Böning, C., and P. Herrmann, Annual cycle of poleward heat transport in the Ocean: Results from high-resolution modeling of the North and Equatorial Atlantic, J. Phys. Oceanogr., 24(1), 91-107, 1994.

Bray, N. A., S. E. Wijffels, J. C. Chong, M. Fieux, S. Hautala, G. Meyers, and W. M. L. Morawitz, Characteristics of the Indo-Pacific Throughflow in the eastern Indian Ocean, Geophys. Res. Letters, 24, 2569-2572, 1997.

Broecker, W. S., "NO", a conservative water-mass tracer, Earth and Planetary Science Letters, 23(1), 100-107, 1974.

Broecker, W. S., and T.-H. Peng, Tracers in the Sea. Palisades, N.Y., Columbia University, 690 p., 1982.

Bryden, H. L., D. H. Roemmich, and J. A. Church, Ocean heat transport across $24^{\circ} \mathrm{N}$ in the 
Pacific, Deep-Sea Res., 38(297-324), 1991.

Bryden, H. L., M. J. Griffiths, A. M. Lavin, R. C. Millard, G. Parrilla, and W. M. Smethie, Decadal changes in water mass characteristics at $24^{\circ} \mathrm{N}$ in the subtropical North Atlantic Ocean, J. Climate, 9(12), 3162-3186, 1996.

Buesseler, K. O., Do upper-ocean sediment traps provide an accurate record of particle flux, Nature, 353, 420-423, 1991.

Buesseler, K. O., The decoupling of production and particulate export in the surface ocean, $J$. Geophys. Res., 12(2), 297-310, 1998.

Chereskin, T. K., Direct evidence for an Ekman balance in the California Current, J. Geophys. Res., 100(C9), 18261-18269, 1995.

Chereskin, T. K., and D. Roemmich, A comparison of measured and wind-driven Ekman transport at $11^{\circ} \mathrm{N}$ in the Atlantic Ocean, J. Phys. Oceanogr., 21, 869-878, 1991.

Chiswell, S. M., J. Toole, and J. Church, Transports across the Tasman Sea from WOCE repeat sections: the East Australian Current 1990-94, New Zeal. J. Mar. Fresh., 31(4), 469-475, 1997.

Clarke, R. A., R. M. Hendry, I. Yashayaev, and D. R. Watts, A western boundary current array in the North Atlantic near $42^{\circ} \mathrm{N}$, Intl. WOCE News. (Unpublished manuscript), (33), 33-34, 1998.

Coachman, L. K., and K. Aagaard, Transports through the Bering Strait: annual and interannual variability, J. Geophys. Res., 93, 15535-15539, 1988.

Coatanoan, C., B. Coste, N. Metzl, M. Fieux, and A. G. Ilahude, Nutrients distribution in the Indonesian straits and along the Sumatra and Java islands during the southern monsoon, Oceanol. Acta, In press, 1999.

Curry, R. G., M. S. McCartney, and T. M. Joyce, Oceanic transport of subpolar climate signals to mid-depth subtropical waters, Nature, 391(6667), 575-577, 1998.

da Silva, A., C. Young, and S. Levitus, Revised surface marine fluxes over the global oceans: the UWM/COADS data set, in WRCP workshop on air-sea flux fields, edited by IFM-Kiel, pp. 13-18. DYNAMO, 1997: Dynamics of North Atlantic Models, Scientific report N2, MAST2, 1996.

de las Heras, M., and R. Schlitzer, On the importance of intermediate water flows for the global ocean overturning, J. Geophys. Res., 104(C7), 15515-15536, 1999.

DeMaster, D. J., The supply and accumulation of silica in the marine environment, Geochim. Cosmochim. Acta, 45, 1715 -1732, 1981.

DeMaster, D. J., T. M. Nelson, S. L. Harden, and C. A. Nittrouer, The cycling and accumulation of biogenic silica and organic carbon in Antarctic deep-sea and continental margin 
environments., Marine chemistry, 35, 489-502, 1991.

Deuser, W. G., T. D. Jickells, P. King, and J. A. Commeau, Decadal and annual changes in biogenic opal and carbonate fluxes in the deep Sargasso Sea, Deep-Sea Res., 42(11/12), 1923-1932, 1995.

Dickson, R. R., Flow statistics from long-term current-meter moorings, the global data set in January 1989, Tech. Rep. WOCE Report No. 46/90, WOCE International Project Office, MAFF Directorate of Fisheries Research, Lowestoft, Suffolk NR33 OHT, England, 1989.

DiMarco, S. F., Nowlin, W. D. Jr., and P. Chapman, Properties and transport of the Mozambique channel, in 1998 U.S. WOCE report., pp. 34-35, U.S. WOCE Office, Dept. of Oceanography, TEXAS A\&M University, College Station, TX 77843-3146. U.S. WOCE Implementation report number 10, 1998.

Donguy, J.-R., and B. Piton, The Mozambique Channel revisited, Oceanologica Acta, 14(6), $546-558,1991$.

ECMWF research department, ECMWF forecast model physical parametrization, Meteorological Bulletin, Shinfield Park, Reading, Berkshire RG2 9AX, England, Fax (0734) 869450, 3rd ed., 1991.

Edmond, J. M., On the Dissolution of carbonate and silicate in the deep ocean, Deep-Sea Res., 21, 455 $480,1974$.

Eriksen, C. C., Geostrophic Equatorial deep jets, J. Mar. Res., 40, Supplement, 143-157, 1982.

Fieux, M., F. Schott, and J. C. Swallow, Deep boundary currents in the western Indian ocean revisited, Deep-Sea Res., 33(4), 415-426, 1986.

Fieux, M., C. Andrié, P. Delecluse, A. G. Ilaude, A. Kartavtseff, F. Mantisi, R. Molcard, and J. C. Swallow, Measurements within the Pacific-Indian Oceans throughflow region, Deep-Sea Res., I(41), 1091-1130, 1994.

Fieux, M., R. Molcard, and A. G. Ilahude, Geostrophic transport of the Pacific-Indian Oceans Throughflow, J. Geophys. Res., 101, 12421-12432, 1996.

Fine, R. A., R. Lukas, M. Bingham, M. J. Warner, and R. H. Gammon, The western equatorial Pacific: A water mass crossroads., J. Geophys. Res., 99, 25,063-25,080, 1994.

Fu, L.-L., Observation and models of inertial waves in the deep ocean, Rev. of Geophys. and Space Phys., 19(1), 141-170, 1981.

Fu, L. L., Mass, heat and freshwater fluxes in the South Indian Ocean, J. Phys. Oceanogr., 16, 1683-1693, 1986.

Ganachaud, A., C. Wunsch, J. Marotzke, and J. Toole, The meridional overturning and large-scale circulation of the Indian Ocean, J. Geophys. Res., 1999, submitted. 
Garcia, H. E., and L. I. Gordon, Oxygen solubility in seawater: Better fitting equations, Limn. Oceanogr., 37(6), 1307-1312, 1992.

Garnier, E., B. Barnier, L. Siefridt, and K. Béranger, Air-Sea fluxes from 15 years of ECMWF reanalysis: A surface boundary condition for OGCMs ?, Climate Dyn., Submitted, 1999.

Garrett, C., and W. Munk, Space-time scales of internal waves, Geophysical Fluid Dynamics, 3, $225-264,1972$.

Garzoli, S. L., A. L. Gordon, V. Kamenkovich, D. Pillsbury, and C. DuncombeRae, Variability and sources of the southeastern Atlantic circulation, J. Mar. Res., 54(6), 1039-1071, 1996.

Georgi, D. T., and J. M. Toole, The Antarctic Circumpolar Current and the oceanic heat and freshwater budget, J. Mar. Res., 40S, 183-197, 1982.

Gill, A. E., Atmosphere-ocean dynamics. Academic Press, 1982.

Gill, A. E., and P. P. Niller, The theory of seasonal variability in the ocean, Deep-Sea Res., 20, 141-178, 1973.

Gille, S. T., Mass, heat and salt transport in the southeastern Pacific: A Circumpolar Current inverse model, J. Geophys. Res., 104(C3), 5191-5209, 1999.

Gleckler, P. J., and B. C. Weare, Uncertainties in global ocean surface heat flux climatologies derived from ship observations, J. Climate, 10(11), 2764-2781, 1997.

Godfrey, J. S., The effect of the Indonesian throughflow on ocean circulation and heat exchange with the atmosphere: A review, J. Geophys. Res., 101, 12217-12237, 1996.

Gordon, A. L., General ocean circulation, in Numerical models of ocean circulation, pp. 39-53. National Academy of Science, Washington, D. C. loads, lkohe ed., 1975.

Gordon, A. L., Interocean exchange of thermocline water, J. Geophys. Res., 91, 5037-5046, 1986.

Gordon, A. L., and R. D. Susanto, Makassar Strait transport: preliminary Arlindo results from MAK-1 and MAK-2, Int. WOCE Newsletter, unpublished manuscript, 1998.

Greatbatch, R. J., A. F. Fanning, A. D. Goulding, and S. Levitus, A diagnosis of interpentadal circulation changes in the North Atlantic, J. Geophys. Res., 96(C12), 22,009-22,023, 1991.

Gründlingh, M. L., On the winter flow in the southern Mozambique Channel, Deep-Sea Res., 40(2), 409-418, 1993.

Hall, M., M. McCartney, and J. A. Whitehead, Antarctic Bottom Water flux in the equatorial Western Atlantic, J. Phys. Oceanogr., 27, 1903-1926, 1997.

Hall, M. M., and H. L. Bryden, Direct estimates and mechanisms of ocean heat transport, Deep-Sea Res., 29(3A), 339-359, 1982.

Hastenrath, S., On meridional heat transports in the world ocean, J. Phys. Oceanogr., 13(12), 922-927, 1982. 
Hastenrath, S., and L. Greischar, The monsoonal heat budget of the hydrosphere-atmosphere system in the Indian Ocean sector, J. Geophys. Res., 98(C4), 6869-6881, 1993.

Hogg, N., P. Biscaye, W. Gardner, and Schmitz, W. J. Jr., On the transport and modification of Antarctic Bottom Water in the Vema Channel, J. Mar. Res., 40, 231-263, Supplement, 1982.

Hogg, N. G., and W. B. Owens, Direct measurements of the deep circulation within the Brazil Basin, Deep-Sea Res., 46(1-2), 335-353, Part II, 1999.

Hogg, N. G., G. Siedler, and W. Zenk, Circulation and variability at the southern boundary of the Brazil Basin, J. Phys. Oceanogr., 29(2), 145-157, 1999.

Holfort, J., and G. Siedler, The oceanic transports of heat and nutrients in the South Atlantic, submitted manuscript, 1999.

Holfort, J., K. M. Johnson, B. Schneider, G. Siedler, and D. W. R. Wallace, Meridional transport of dissolved inorganic carbon in the South Atlantic Ocean, Global Biogeochemical Cycles, 13(1), 253-254, 1999b.

Honjo, S., and S. Manganini, Annual biogenic particle fluxes to the interior of the North Atlantic Ocean-studied at 24N 21W and 48N 21W, Deep-Sea Res., 40, 587-608, Part II, 1993.

Hsiung, J., Estimates of global oceanic meridional heat trandport, J. Phys. Oceanogr., 15(11), 1405-1413, 1985.

Hu, D. M., Global-scale water masses, meridional circulation, and heat transport simulated with a global isopycnal ocean model, J. Phys. Oceanogr., 27(1), 96-120, 1997.

Huang, B., and J. Shukla, A comparison of two surface wind stress analyses over the tropical Atlantic during 1980-1987, Journal of Climate, $9(5)$, 906-927, 1996.

Hufford, G. E., M. S. McCartney, and K. A. Donohue, Northern boundary currents and adjacent recirculations off southwestern Australia, Geophys. Res. Letters, 24(22), 2797-2800, 1997.

Ichikawa, H., and R. C. Beardsley, Temporal and spatial variability of volume transport of the Kuroshio in the East China Sea, Deep-Sea Res., 40, 583-605, 1993.

Jackett, D. R., and T. J. McDougall, A neutral density variable for the world's oceans, J. Phys. Oceanogr., 27(2), 237-263, 1997.

Jacobs, S. S., R. G. Fairbanks, and Y. Horibe, Origin and evolution of water masses near the Antarctic continental margin, in Oceanology of the Antarctic Continental Shelf, edited by S. S. Jacobs, vol. 43 of Antarctic Research Series, pp. 59-85. American Geophysical Union, 1985.

Jayne, S. R., Dynamics of global ocean heat transport variability, Ph.D. thesis, Mass. Inst. of Technol. / Woods Hole Oceanog. Inst. Joint Program, Cambridge, Mass., USA, 150 pp., 1999.

Jayne, S. R., and R. Tokmakian, Forcing and sampling of ocean general circulation models: impact of high-frequency motions, J. Phys. Oceanogr., 27(6), 1173-1179, Notes and 
correspondences, 1997.

Jenkins, W., and J. Goldman, Seasonal cycling and primary production in the Sargasso Sea, J. Mar. Res., 43, 465-491, 1985.

Jenkins, W. J., Tritium and ${ }^{3}$ He in the Sargasso Sea, J. Mar. Res., 38, 533-569, 1980.

Jenkins, W. J., ${ }^{3} \mathrm{H}$ and ${ }^{3} \mathrm{He}$ in the Beta Triangle: Observations of gyre ventilation and oxygen utilization rates, J. Phys. Oceanogr., 17, 763-781, 1987.

Johns, E., D. R. Watts, and H. T. Rossby, A test of geostrophy in the Gulf Stream, J. Geophys. Res., $94(\mathrm{C} 3), 3211-3222,1989$.

Johns, W. E., T. N. Lee, F. A. Schott, R. J. Zantopp, and R. H. Evans, The North Brazil Current retroflexion: seasonal structure and eddy variability, J. Geophys. Res., 95(C12), $22,103-22,120,1990$.

Johns, W. E., T. N. Lee, R. C. Beardsley, J. Candela, R. Limeburner, and B. Castro, Annual cycle and variability of the North Brazil Current, J. Phys. Oceanogr., 28(1), 103-128, 1998.

Johns, W. E., T. N. Lee, D. Zhang, R. Zantopp, Liu, C.-T., and Y. Yang, The Kuroshio east of Taiwan: moored transport observations from the WOCE PCM-1 array, in preparation, 1999.

Johnson, G. C., D. L. Musgrave, B. A. Warren, A. Ffield, and D. B. Olson, Flow of bottom and deep water in the Amirante Passage and Mascarene Basin, J. Geophys. Res., 103(C13), 30,973-30,984, 1998.

Josey, S. A., E. C. Kent, and P. K. Taylor, New insights into the ocean heat budget closure problem from analysis of the SOC air-sea flux climatology, J. Climate, 9, 2856-2880, 1999.

Jourdan, D., P. Peterson, and C. Gautier, Oceanic freshwater budget and transport as derived from satellite radiometric data, J. Phys. Oceanogr., 27(3), 457-467, 1997.

Joyce, T., and C. Corry, WOCE operations manual, Tech. Rep. WOCE report No.67/91 / WHPO publication 90-1, Revision 2, Woods Hole Oceanographic Institution, Woods Hole, Mass., USA, 1994.

Kalnay, E., and and 21 others, The NCEP/NCAR 40-year reanalysis project, Bulletin of Amer. Meteo. Soc., $77(3), 437-471,1996$.

Kawai, H., Hydrography of the Kuroshio extention, in Kuroshio: its physical aspects (H. Stommel and K. Yoshida Ed.), pp. 335-352. University of Tokyo Press, 518pp., 1972.

Keeling, R. F., and T.-H. Peng, Transport of heat, $\mathrm{CO}_{2}$ and $\mathrm{O}_{2}$ by the Atlantic's thermohaline circulation, Phil. Trans. R. Soc. Lond., 348B, 133-142, 1995.

Keith, D. W., Meridional energy transport: uncertainty in zonal means, Tellus, 47A, 30-44, 1995.

Killworth, P. D., D. Stainforth, D. J. Webb, and S. M. Paterson, The development of a free surface Bryan-Cox-Semtner ocean model, J. Phys. Oceanogr., 21, 1333-1348, 1991. 
Koltermann, K. P., A. Sokov, V. Terechtchencov, S. Dobroliubov, K. Lorbacher, and A. Sy, Decadal changes in the thermohaline circulation of the North Atlantic, Deep-Sea Res., 46(1+2), 109-138, Part II, 1999.

Lampitt, R. S., and A. N. Antia, Particle flux in deep seas: regional characteristics and temporal variability, Deep-Sea Res., (I) 44(8), 1377-1403, 1997.

Lavin, A., Climatic changes in temperature and salinity in the subtropical North Atlantic, Master's thesis, Massachusetts Institute of Technology, Departement of Earth, Atmospheric and Planetary Sciences, 1993.

Lee, T., and J. Marotzke, Inferring meridional mass and heat transports of the Indian Ocean by fitting a general circulation model to climatological data, J. Geophys. Res., 102(C5), $10,585-10,602,1997$.

Lee, T., and J. Marotzke, Seasonal cycles of meridional overturning and heat transport of the Indian Ocean, J. Phys. Oceanogr., 28(5), 923-943, 1998.

Lee, T. N., W. E. Johns, R. J. Zantopp, and E. R. Fillenbaum, Moored observations of western boundary current variability and thermohaline circulation at 26.5 degrees $\mathrm{N}$ in the subtropical North Atlantic, J. Phys. Oceanogr., 26(6), 962-983, 1996.

Lee, T. N., W. E. Johns, C.-T. Liu, D. Zhang, R. Zantopp, and Y. Yang, The Kuroshio east of Taiwan; Current structure and transport on daily to seasonal time scales, J. Phys. Oceanogr., submitted, 1999.

Lemke, P., The expedition Antarktis X/4 of RV Polarstern in 1992, Tech. Rep. 140, Berichte zur Polarforschung, 90pp., 1994.

Levitus, S., Ekman volume fluxes for the world ocean and individual ocean basins, J. Phys. Oceanogr., 18, 271-279, 1988.

Levitus, S., R. Burgett, and T. Boyer, World Ocean Atlas 1993, vol. 3, Salinity, vol. 4, Temperature, NOAA Atlas NESDIS 3 \& 4, U.S. Dep. of Comm., Washington D.C., 1994.

Lisitzin, A., Sedimentation in the world ocean, Soc. of Paleont. and Min. Special publ., 17, 1972.

Lutjeharms, J. R. W., The Agulhas Current system during the northeast monsoon season, $J$. Phys. Oceanogr., 6, 665-670, 1976.

Lux, M., Franchissement de l'Equateur par les masses d'eau dans le cadre de la circulation thermohaline, Ph.D. thesis, Université de Bretagne Occidentale, Laboratoire de Physique des Océans, IFREMER centre de Brest, BP70, 29280, Plouzané, France, 1997.

Luyten, J., H. Stommel, and C. Wunsch, A diagnostic study of the northern Atlantic subpolar gyre, J. Phys. Oceanogr., 15(10), 1344-1348, 1985.

Macdonald, A., Oceanic fluxes of mass, heat and freshwater: A global estimate and perspective, 
Ph.D. thesis, Department of Earth, Atmospheric and Planetary Sciences, Massachusetts Institute of Technology, Cambridge 02139, 1995.

Macdonald, A., The global ocean circulation: a hydrographic estimate and regional analysis, Prog. Oceanogr., 41, 281-382, 1998.

Macdonald, A. M., and C. Wunsch, An estimate of global ocean circulation and heat fluxes, Nature, 382(6590), 436-439, 1996.

Manabe, S., and R. J. Stouffer, Two stable equilibria of a coupled ocean-atmosphere model, $J$. Climate, 1(9), 841-866, 1988.

Marotzke, J., Analysis of thermohaline feedback, in Decadal Climate variability, dynamics and predictability, edited by D. L. T. Anderson, and J. Willebrand, vol. I 44 of NATO SAI. Springer-Verlag, 1996.

Marotzke, J., and J. Willebrand, The North Atlantic mean circulation: combining data and dynamics. The warmwatersphere of the North Atlantic Ocean. W. Krauss (ed.), chap. 3. Gebüder Borntraeger, Berlin-Stuttgart, 1996.

Martel, F., and C. Wunsch, The North Atlantic circulation in the early 1980's-an estimate from inversion of a finite difference model, J. Phys. Oceanogr., 23, 898-924, 1993.

McCartney, M. S., and R. A. Curry, Transequatorial flow of Antarctic Bottom Water in the western Atlantic Ocean: abyssal geostrophy at the Equator, J. Geophys. Res., 23, 1264-1276, 1993.

McDougall, T., Neutral surfaces, J. Phys. Oceanogr., 17, 1950-1964, 1987.

McDougall, T. J., Parameterizing mixing in inverse models, in Parameterization of small-scale processes, Hawaiian winter workshop, University of Hawaii at Manoa. Aha Hulikoa, 1991.

McIntosh, P. C., and S. R. Rintoul, Do inverse box models work?, J. Phys. Oceanogr., 27(2), 291-308, 1997.

McManus, J., D. E. Hammond, W. M. Berelson, T. E. Kilgore, D. J. Demaster, O. G.

Ragueneau, and R. W. Collier, Early diagenesis of biogenic opal: Dissolution rates, kinetics and paleoceanographic implications, Deep-Sea Res., 42(2-3), 871-903, 1995.

McTaggart, K. E., and G. C. Johnson, CTD/O2 measurements collected on a Climate and Global Change cruise (WOCE sections P14S and P15S) during January-March 1996, Tech. rep., NOAA Data Report ERL PMEL-63, http://toast.pmel.noaa.gov/admin/scripts/publications.plx?number=1895, 485pp., 1997.

Ménaché, M., Première campagne océanographique du Commandant Robert Giraud dans le canal de Mozambique, 11 octobre au 28 novembre 1957, Cahiers Océanographiques, 15, 224-237, 1963. 
Mercier, H., Determining the general circulation of the ocean: a nonlinear inverse problem, $J$. Geophys. Res., 91(C4), 5103-5109, 1986.

Mercier, H., and K. Speer, Transport of bottom water in the Romanche Fracture Zone and the Chain Fracture Zone, J. Phys. Oceanogr., 28(5), 779-790, 1998.

Mercier, H., M. Ollitrault, and P. Y. Le Traon, An inverse model of the North Atlantic general circulation using lagrangian float data, J. Phys. Oceanogr., 23, 689-715, 1993.

Mestas-Nuñez, A. M., D. B. Chelton, M. H. Freilich, and J. G. Richman, An evaluation of ECMWF-based climatological wind-stress fields, J. Phys. Oceanogr., 24, 1532-1549, 1994.

Meyers, G., R. J. Bailey, and A. P. Worby, Geostrophic transport of Indonesian throughflow, Deep-Sea Res., 42(7), 1163-1174, 1995.

Michaels, A. F., N. R. Bates, K. O. Buesseler, C. A. Carlson, and A. H. Knap, Carbon-cycle imbalances in the Sargasso Sea, Nature, 372, 537-540, 1994.

Minster, J.-F., and M. Boulahdid, Redfield ratios along isopycnal surfaces-a complementary study, Deep-Sea Res., 34(12), 1981-2003, 1987.

Muller, T. J., Y. Ideka, N. Zangenberg, and L. V. Nonato, Direct measurements of western boundary currents off Brazil between $20^{\circ} \mathrm{S}$ and $28^{\circ} \mathrm{S}$, J. Geophys. Res., 103(C3), 5429-5437, 1998.

Munk, W., Internal waves. Evolution of Physical Oceanography, Scientific surveys in honor of Henry Stommel. B. A. Warren and C. Wunsch (eds.), pp. 264-291. The MIT Press, Cambridge, MA, 1981.

Munk, W., and C. Wunsch, The Moon and mixing: abyssal recipes II, Deep-Sea Res., 45, part I, $1977-2010,1998$.

Munk, W. H., Internal wave spectra at the buoyant and inertial frequencies, J. Phys. Oceanogr., 10, 1718-1728, 1980.

Musgrave, D. L., J. Chou, and W. J. Jenkins, Application of a model of upper-ocean physics for studying seasonal cycles of oxygen, J. Geophys. Res., 93, 15679-15700, 1988.

Nelson, D. M., P. Tréguer, M. A. Brezezinski, A. Leynaert, and B. Queguiner, Production and dissolution of biogenic silica in the ocean: Revised gobal estimates, comparison with regional data and relationship to biogenic sedimentation, Global Biogeochemical Cycles, 9, 359-372, 1995.

Niiler, P. P., On the Ekman divergence in an oceanic jet, J. Geophys. Res., 28, 7048-7052, 1969.

Oberhuber, J. M., An atlas based on the COADS data set: the budgets of heat, buoyancy and turbulent kinetic energy at the surface of the global ocean, Tech. rep., Max-Planck-Inst. fur Meteorol., Hamburg, Germany, 1988. 
Orsi, A. H., G. C. Johnson, and J. L. Bullister, Circulation, mixing and production of Antarctic Bottom Water, Prog. Oceanogr., 43(1), 55-109, 1999.

Oudot, C., P. Morin, F. Baurand, M. Wafar, and P. L. Corre, Northern and southern water masses in the equatorial Atlantic sector: results of nutrients from the CITHER-1 cruise (WOCE A6 and A7 lines), Deep-Sea Res., 45, part I(6), 873-902, 1998.

Parilla, G., A. Lavin, H. Bryden, M. Garcia, and R. Millard, Rising temperature in the subtropical North Atlantic over the past 35 years, Nature, 369, 48-51, 1994.

Park, Y.-H., E. Charriaud, P. Craneguy, and A. Kartavtseff, Frontal structure, water masses and transports between Africa and Antarctica, J. Geophys. Res., Submitted, 1999.

Pedlosky, J., Geophysical Fluid Dynamics. 2nd ed. Springer-Verlag, 710pp, 1987.

Peng, T.-H., T. Takahashi, and W. S. Broecker, Seasonal variability of carbon dioxide, nutrients and oxygen in the northern North Atlantic surface water: Observations and a model, Tellus, 39B, 439-458, 1987.

Polzin, K. L., J. M. Toole, G. R. Ledwell, and R. W. Schmitt, Spatial variability of turbulent mixing in the abyssal ocean, Science, 276, 93-96, 1997.

Price, J. R., R. A. Weller, and R. R. Schudlich, Wind-driven ocean currents and Ekman transport, Science, 238(4833), 1534-1538, 1988.

Redfield, A. C., B. H. Ketchum, and F. A. Richards, The influence of organisms on the composition of sea water, The Sea, vol. 2, pp. 26-77. Interscience, N.Y. (M. N. Hill ed.), 1963.

Reid, J. L., Jr., Intermediate waters of the Pacific Ocean, no. 2 in The Johns Hopkins Oceanographic studies. The Johns Hopkins Press, Baltimore, 1965.

Reid, J. L., Jr., Northwest Pacific Ocean waters in winter, in Johns Hopkins Oceanographic Studies, vol. 5. Woods Hole Oceanographic Institution, 1973.

Reid, J. L., Jr., On the total geostrophic circulation of the North Atlantic Ocean: Flow patterns, tracers, and transports, Prog. Oceanogr., 33, 1-92, 1994.

Reid, J. L., Jr., On the circulation of the South Atlantic Ocean, in The South Atlantic: past and present circulation, edited by G. Wefer, W. H. Berger, G. Siedler, and D. J. Webb. Springer, 1996.

Reverdin, G., D. Cayan, and Y. Kushnir, Decadal variability of hydrography in the upper North Atlantic, J. Geophys. Res., 102(C4), 8505-8531, 1997.

Richardson, P. L., and Schmitz, W. J. Jr., Deep cross-equatorial flow in the Atlantic measured with SOFAR floats, J. Geophys. Res., 98(C5), 8371-8387, 1993.

Riley, G. A., Oxygen, phosphate and nitrate in the Atlantic Ocean, Bulleting of the Bingham Oceanography Collection, 12, 169 pp., 1951. 
Rintoul, S. R., Mass, heat and nutrient fluxes in the Atlantic Ocean determined by inverse methods, Ph.D. thesis, Massachusetts Institute of Technology, 287 pp., 1988.

Rintoul, S. R., South Atlantic interbasin exchange, J. Geophys. Res., 96, 2675-2592, 1991.

Rintoul, S. R., On the origin and influence of Adélie Land bottom water, in Ocean, ice and atmosphere: interactions at the Antarctic continental margin, edited by S. Jacobs, and R. Weiss, vol. 75 of Antarctic Research Series, pp. 151-171. American Geophysical Union, 1998.

Rintoul, S. R., and J. L. Bullister, A late winter hydrographic section from Tasmania to Antarctica, Deep-Sea Res., 46(9), 1417-1454, 1999.

Rintoul, S. R., and C. Wunsch, Mass, heat, oxygen and nutrient fluxes and budget in the North Atlantic Ocean, Deep-Sea Res., 38, Suppl., 355-377, 1991.

Ripa, P., "Inertial" oscillations and the $\beta$-plane approximation(s), J. Phys. Oceanogr., 27(5), 633-647, 1997.

Robbins, P. E., and H. L. Bryden, Direct observations of advective nutrient and oxygen fluxes at $24^{\circ} \mathrm{N}$ in the Pacific Ocean, Deep-Sea Res., 41, part I(1), 143-168, 1993.

Robbins, P. E., and J. M. Toole, The dissolved silica budget as a constraint on the meridional overturning circulation in the Indian Ocean, Deep-Sea Res., 44(5), 879-906, 1997.

Roemmich, D., and T. McCallister, Large scale circulation of the North Pacific Ocean, Prog. Oceanogr., 22, 171-204, 1989.

Roemmich, D., and C. Wunsch, Two transatlantic sections: meridional circulation and heat flux in the subtropical North Atlantic Ocean, Deep-Sea Res., 32(6), 119-664, 1985.

Roemmich, D., T. McCallister, and J. Swift, A trans-Pacific hydrographic section at, $24^{\circ} \mathrm{N}$ : the distribution of properties in the subtropical gyre, Deep-Sea Res., 38(1A), 1-20, Suppl., 1991.

Roemmich, D., S. Hautala, and D. Rudnick, Northward abyssal transport through the Samoan passage and adjacent regions, J. Geophys. Res., 101(C6), 14,039-14,055, 1996.

Roether, W., R. Schlitzer, A. Putzka, P. Beining, K. Bulsiewicz, G. Rohardt, and F. Delahoyde, A chlorofluoromethane and hydrographic section across Drake Passage-deep water ventilation and meridional property transport, J. Geophys. Res., 98(C8), 14423-14435, 1993.

Rosenberg, M., S. Bray, N. Bindoff, S. Rintoul, N. Johnson, S. Bell, and P. Towler, Aurora Australis Marine Science Cruises AU9501, AU9604 and AU9601-Oceanographic field measurements and analysis, inter-cruise comparisons and data quality notes, Tech. rep., Antarctic Cooperative Research Centre, 12, 1997.

Sætre, R., and A. J. da Silva, The circulation in the Mozambique Channel, Deep-Sea Res., 31(5), 485-508, 1984. 
Sarmiento, J. L., and J. R. Toggweiler, A new model for the role of the oceans in determining atmospheric $P_{\mathrm{CO}_{2}}$, Nature, 308, 621-624, 1984.

Sarmiento, J. L., G. Thiele, R. M. Key, and W. S. Moore, Oxygen and nitrate new production and remineralization in the North-Atlantic subtropical gyre, J. Geophys. Res., 95(C10), 18303-18315, 1990.

Sarmiento, J. L., J. C. Orr, and U. Siegenthaler, A perturbation simulation of $\mathrm{CO}_{2}$ uptake in an ocean general circulation model, J. Geophys. Res., 97(C3), 3621-3645, 1992.

Saunders, P. M., Accuracy of measurement of salinity, oxygen, and temperature in the deep ocean, J. Phys. Oceanogr., 16(1), 189-195, 1986.

Saunders, P. M., and B. B. King, Oceanic fluxes on the WOCE A11 Section, J. Phys. Oceanogr., 25(9), 1942-1958, 1995.

Sayles, F. L., W. G. Deuser, J. E. Goudreau, W. H. Dickinson, T. D. Jickells, and P. King, The benthic cycle of biogenic opal at the Bermuda Atlantic Time Series site, Deep-Sea Res., pp. 1-27, 1996.

Schlitzer, R., Modelling the nutrient and carbon cycles of the North Atlantic, J. Geophys. Res., 93, 10699-10723, 1988.

Schlitzer, R., Determining the mean, large-scale circulation of the Atlantic with the adjoint method, J. Phys. Oceanogr., 23, 1935-1952, 1993.

Schludlich, R. R., and J. F. Price, Observations of seasonal variation in the Ekman layer, $J$. Phys. Oceanogr., 28(6), 1187-1204, 1998.

Schmitt, R. W., P. S. Bogden, and C. Dorman, Evaporation minus precipitation and the density fluxes for the North Atlantic, J. Phys. Oceanogr., 19, 1208-1221, 1989.

Schmitz, W. J. Jr., On the World Ocean Circulation: Volume I: Some global features/ North Atlantic Circulation, Tech. Rep. WHOI-96-03, Woods Hole Oceanographic Institution, Woods Hole, Massachusetts 02543, 1996a.

Schmitz, W. J. Jr., On the World Ocean Circulation: Volume II: The Pacific and Indian Oceans / A global update, Tech. Rep. WHOI-96-08, Woods Hole Oceanographic Institution, Woods Hole, Massachusetts 02543, 1996b.

Schmitz, W. J. Jr., and M. S. McCartney, On the North Atlantic circulation, Rev. of Geophys., 31(1), 29-49, 1993.

Schott, F., J. C. Swallow, and M. Fieux, The Somali Current at the Equator: annual cycle of currents and transports in the upper $1000 \mathrm{~m}$ and connection to neighbouring latitudes, Deep-Sea Res., 37(12A), 1825-1848, 1990.

Schott, F. A., T. N. Lee, and R. Zantopp, Variability of structure and transport of the Florida 
Current in the period range of day to seasonal, J. Phys. Oceanogr., 18(9), 1209-1230, 1988a.

Schott, F. S., M. Fieux, J. Kindle, J. Swallow, and R. Zantopp, The boundary currents east of Madagascar. 2. Direct measurements and model comparisons, J. Geophys. Res., 93(C5), 4963-4974, 1988.

Semtner, A. J., Advanced physical oceanographic numerical modelling, chap. Finite-difference formulation of a world ocean model, pp. 187-202. D. Reidel Publishing Company (O'Brien ed.), 1986.

Semtner, A. J., and R. M. Chervin, Ocean general circulation from a global eddy-resolving model, J. Geophys. Res., 97, 5493-5550, 1992.

Shannon, L. V., and G. Nelson, The Benguela: large scale features and processes and system variability, pp. 163-210. Springer, in: The South Atlantic: past and present circulation (Wefer, Berger, Siedler and Webb ed.), 1996.

Shriver, J. F., and H. E. Hulburt, The contribution of the global thermohaline circulation to the Pacific and Indian throughflow via Indonesia, J. Geophys. Res., 102(C3), 5491-5511, 1997.

Siedler, G., T. J. Mueller, R. Onken, M. Arhan, H. Mercier, B. A. King, and P. M. Saunders, The zonal WOCE sections in the South Atlantic, pp. 83-104. Springer-Verlag, Berlin, in: The South Atlantic: past and present circulation (Wefer, Berger, Siedler and Webb ed.), 1996.

Siefridt, L., B. Barnier, K. Béranger, and H. Roquet, Evaluation of operational ECMWF surface heat fluxes: impact of parameterization changes during 1986-1995, J. Mar. Syst., 19, 112-135, 1999.

Siegel, D. A., and W. G. Deuser, Trajectories of sinking particles in the Sargasso Sea: modelling of statistical funnels above deep-ocean sediment traps, Deep-Sea Res., 44(9-10), 1519-1541, Part I, 1997.

Sloyan, B. M., and S. R. Rintoul, Estimates of area-averaged diapycnal fluxes from basin-scale budgets, J. Phys. Oceanogr., Submitted, 1999a.

Sloyan, B. M., and S. R. Rintoul, The Southern Ocean limb of the global deep overturning circulation, J. Phys. Oceanogr., Submitted, 1999b.

Sokov, A. V., V. P. Tereschenkov, and P. P. Shirshov, Cruise 36 of RV Professor Shtokman to the North Atlantic, WOCE Intl. News., (31), 39-40, 1998.

Speer, K. G., J. Holfort, T. Reynaud, and G. Siedler, South Atlantic heat transport at $11^{\circ} \mathrm{S}$, pp. 105-120. Springer, in: The South Atlantic: past and present circulation (Wefer, Berger, Siedler and Webb ed.), 1996.

Spitzer, W. S., and W. J. Jenkins, Rates of vertical mixing, gas exchange and new production: estimates from seasonal gas cycles in the upper ocean near Bermuda, J. Mar. Res., 47, 169, 
1989.

Stammer, D., R. Tokmakian, A. Semtner, and C. Wunsch, How well does a $1 / 4^{\circ}$ global circulation model simulate large scale oceanic observations?, J. Geophys. Res., 101(C10), $25,779-25,811,1996$.

Stephens, B. B., R. F. Keeling, M. Heimann, K. D. Six, R. Murnane, and K. Caldeira, Testing global ocean carbon cycle models using measurements of atmospheric $\mathrm{O}_{2}$ and $\mathrm{CO}_{2}$ concentration, Global Biogeochemical Cycles, 12(2), 213-230, 1998.

Stommel, H., and A. B. Arons, On the abyssal circulation of the world ocean-II. An idealized model of the circulation pattern and amplitude in oceanic basins, Deep-Sea Res., 6, 217-233, 1960.

Stommel, H., E. D. Stroup, and B. Warren, Trans-Pacific hydrographic sections at lats. $43^{\circ} \mathrm{S}$ and $28^{\circ}$ S: The Scorpio expedition-I. Preface, Deep-Sea Res., 20, 1-7, 1973.

Stramma, L., and J. Lutjerharms, The flow field in the subtropical gyre of the South Indian Ocean, J. Geophys. Res., 102(C3), 5513-5530, 1997.

Sturges, W., B. G. Hong, and A. J. Clarke, Decadal wind forcing on the North Atlantic subtropical gyre, J. Phys. Oceanogr., 28(4), 659-668, 1998.

Takahashi, K., and S. Honjo, Vertical flux of Radiolaria: A taxon-quantitative sediment trap study from the western tropical Atlantic, Micropaleontology, 27(2), 140-190, 1981.

Talley, L., T. M. Joyce, and R. A. D. Szoeke, Trans-Pacific sections at $47^{\circ} \mathrm{N}$ and $152^{\circ} \mathrm{W}$ : Distribution of properties, Deep-Sea Res., 38(1A), 63-82, Suppl., 1991.

Talley, L. D., Meridional heat transport in the Pacific Ocean, J. Phys. Oceanogr., 14(231-241), 1984 .

Talley, L. D., G. Fryer, and R. Lumpkin, Physical Oceanography of the Tropical Pacific-Geography of the Pacific Islands, chap. 2, pp. 19-32. Bess Press, Honolulu, HI, M. Rapaport ed., 1998.

Talley, L. D., and Roemmich, D. editors, Joseph L. Reid: a tribute in recognition of 40 years of contribution to oceanography, Deep-Sea Res., 38(Suppl. 1A), 654pp, 1991.

Toole, J., and B. A. Warren, A hydrographic section across the Subtropical South Indian Ocean, Deep-Sea Res., 40, 1973-2019, 1993.

Tréguer, P., D. M. Nelson, A. J. van Bennekom, D. J. DeMaster, A. Leynaert, and B. Quéguiner, The silica balance in the World Ocean: A reestimate, Science, 268, 375-379, 1995.

Trenberth, K. E., and A. Solomon, The global heat balance; heat transports in the atmosphere and ocean, Climate Dynamics, 10, 107-134, 1994.

Trenberth, K. E., W. G. Large, and J. G. Olson, The mean annual cycle in global ocean wind 
stress, J. Phys. Oceanogr., 20, 1742-1760, 1990.

Tsimplis, M. N., S. Bacon, and H. L. Bryden, The circulation of the sub-Tropical South Pacific derived from hydrographic data, J. Geophys. Res., 103(C10), 21,443-21,468, 1998.

Wajsowicz, R. C., A relationship between interannual variations in the South Pacific wind stress curl, the Indonesian throughflow, and the western Pacific warm pool, J. Phys. Oceanogr., $24(10), 2180-2187,1994$.

Wajsowicz, R. C., The response of the Indo-Pacific throughflow to interannual variations in the Pacific wind stress. Part II: Realistic geometry and ECMWF wind stress anomalies for 1985-1989, J. Phys. Oceanogr., 26(12), 2589-2610, 1997.

Warren, B. A., Transpacific sections at lats. $43^{\circ} \mathrm{S}$ and $28^{\circ} \mathrm{N}$ : The Scorpio expedition-II. Deep water., Deep-Sea Res., 20, 9-38, 1973.

Warren, B. A., Trans-Indian hydrographic section at lat. 18 degrees S: property distributions and circulation in the South Indian Ocean, Deep-Sea Res., 28(8A), 759-788, 1981.

Warren, B. A., Why is no deep water formed in the North Pacific?, J. Mar. Res., 41, 327-341, 1983.

Warren, B. A., Approximating the energy transport across oceanic sections, J. Geophys. Res., 104(C4), 7915-7919, 1999.

Warren, B. A., and C. G. Johnson, Deep currents in the Arabian Sea in 1987, Mar. Geol., 104, 279-288, 1992.

Watson, A. J., P. D. Nightingale, and D. J. Cooper, Modeling atmosphere-ocean $\mathrm{CO}_{2}$ transfer, Phil. Trans. R. Soc. Lond. B, 348, 125-132, 1995.

Weatherly, G. L., Y. Y. Kim, and E. A. Kontar, Eulerian measurements of the North Atlantic Deep Water deep western boundary current at $18^{\circ} \mathrm{S}$, To be submitted, 1999.

Weller, R. A., and A. J. Plueddemann, Observation of the vertical structure of the oceanic boundary layer, J. Geophys. Res., 101(C4), 8789-8806, 1996.

Whitworth, T., III, W. D. Nowlin Jr., and S. J. Worley, The net transport of the Antarctic Circumpolar Current through Drake Passage, J. Phys. Oceanogr., 12, 960-971, 1982.

Whitworth, T. III, B. A. Warren, Nowlin, W. D. Jr., S. B. Rutz, R. D. Pillsbury, and M. I. Moore, On the deep western-boundary current in the Southwest Pacific Basin, Prog. Oceanogr., 43, 1-54, 1999.

Wijffels, S. E., Exchanges between hemispheres and gyres: a direct approach to the mean circulation of the equatorial Pacific, Ph.D. thesis, Massachusetts Institute of Technology / Woods Hole Oceanographic Institution Joint Program, 297pp, 1993.

Wijffels, S. E., R. W. Schmitt, and H. L. Bryden, Transport of freshwater by the oceans, J. Phys. 
Oceanogr., 22, 155-162, 1992.

Wijffels, S. E., J. M. Toole, H. L. Bryden, R. A. Fine, W. J. Jenkins, and J. L. Bullister, The water masses and circulation at $10^{\circ} \mathrm{N}$ in the Pacific, Deep-Sea Res., 43, 501-544, 1996.

Wijffels, S. E., J. M. Toole, and R. Davis, On the circulation of the subtropical South Pacific, unsubmitted manuscript, 1999.

Wijffels, S. W., E. Firing, and H. L. Bryden, Direct observations of the Ekman balance at $10^{\circ} \mathrm{N}$ in the Pacific, J. Phys. Oceanogr., 24, 1666-1679, 1994.

Williams, R. G., and M. J. Follows, The Ekman transfer of nutrients and maintenamce of new production over the North Atlantic, Deep-Sea Res., 45(2-3), 461-489, 1998.

Worthington, L. V., and H. Kawai, Comparison between deep sections across the Kuroshio and the Florida Current and Gulf Stream, in Kuroshio: its physical aspects (H. Stommel and K. Yoshida Ed.), pp. 371-386. University of Tokyo Press, 518pp., 1972.

Wunsch, C., The North Atlantic general circulation West of $50^{\circ} \mathrm{W}$ determined by inverse methods, Rev. of Geophys. and Space Phys., 16(4), 583-620, 1978.

Wunsch, C., Dynamically consistent hydrography and absolute velocity in the eastern North Atlantic Ocean, J. Geophys. Res., 99(C7), 14,071-14,090, 1994.

Wunsch, C., The Ocean Circulation Inverse Problem. Cambridge U. Press., Cambridge, 437 pp, 1996.

Wunsch, C., and B. Grant, Towards the general circulation of the North Atlantic Ocean, Prog. Oceanogr., 11, 1-59, 1982.

Wunsch, C., D. Hu, and G. Barbara, Mass, heat, salt and nutrient fluxes in the South Pacific Ocean, J. Phys. Oceanogr., 13(5), 725-753, 1983.

Wüst, G., Florida-und Antillenstrom. Berlin U., Institut f. Meereskunde, Veröff, N. F., A. Geogr.-naturwiss. Reihe, Heft 12, 48pp, 1924.

Wyrtki, K., Oceanographic atlas of the international Indian Ocean expedition. Amering Publishing Co. Pvt. Ltd., New Delhi, 1970, reprinted in 1988.

Zang, X., Spectral description of low frequency oceanic variability, Ph.D. thesis, Massachusetts Institute of Technology, MIT/WHOI Joint Program in Physical Oceanography, 54-911, 77 Mass Ave., Cambridge, MA02139, 1999, in preparation.

Zemba, J. C., The structure and transport of the Brazil Current between $27^{\circ}$ and $36^{\circ}$ South, Ph.D. thesis, Woods Hole Oceanographic Institution / Massachusetts Institute of Technology, Woods Hole, MA 02543, WHOI-91-37, 160pp., 1991.

Zenk, W., G. Siedler, B. Lenz, and N. G. Hogg, Antarctic Bottom Water flow through the Hunter Channel, J. Phys. Oceanogr., 29, 2785-2801, 1999. 